\title{
Workshop on Raman Spectroscopy in Optical and Materials Sciences
}

National Institute of Standards and Technology

Optical Technology Division Gaithersburg, MD

Edited by:

Alfons Weber

Optical Technology Division

QC 



\section{Workshop on Raman Spectroscopy in Optical and Materials Sciences}

National Institute of Standards and Technology Optical Technology Division

Gaithersburg, MD December 10, 1996

Edited by:

Alfons Weber

Optical Technology Division

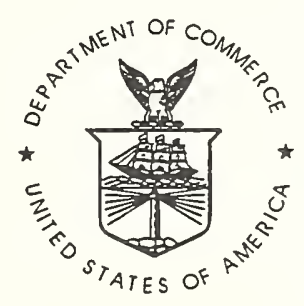

U.S. DEPARTMENT OF COMMERCE Michael Kantor, Secretary

TECHNOLOGY ADMINISTRATION

Mary L. Good, Under Secretary for Technology

NATIONAL INSTITUTE OF STANDARDS 



\section{Executive Summary}

Raman spectroscopy is done in many units of NIST, these activities having in some cases been established more than 20 years ago. Most of them are pursued in the Chemical Sciences and Technology Laboratory with several others in the Materials Science and Engineering Laboratory and the Electronics and Electrical Engineering Laboratory. The Physics Laboratory is the latest major operating unit to set up a program in Raman spectroscopy. Located in the Optical Materials and Infrared Properties Group of the Optical Technology Division, the program was started with surplus equipment in the spring of 1995 and became an effective research unit with the installation of state-of-theart apparatus in March 1996. A distinguishing feature of this effort is the availability of a high-field superconducting solenoid magnet which allows the study of Raman spectra in the presence of a magnetic field. These facilities comprise the Magneto-Raman Spectroscopy Laboratory. Based on these facilities a research program was established to study the Raman spectra of solid state materials which are at the forefronts of current scientific and technological interest. The initial emphasis was given to superconducting materials with high critical transition temperatures (high- $T_{C}$ superconductors) and to those which exhibit a giant magneto resistance effect.

The Magneto-Raman Spectroscopy Laboratory is also intended to serve as a user facility for NIST staff and outside investigators. This service is certainly available to those who wish to have information obtained through Raman spectroscopy but do not have their own equipment. However, even when such equipment is available, this may not be adequate to serve the investigator's specific needs. The present laboratory facility will met these needs since a user laboratory is not available elsewhere at NIST.

In order to better serve industrial and technological needs and to assist the NIST community in being aware of the availability of a user laboratory a Workshop on Raman Spectroscopy in Optical and Materials Sciences was convened on December 10, 1996. The format was a series of lectures with follow up discussions. Speakers were solicited from industry, academia, and government laboratories to represent a cross section of current research interests.

The presentations covered research done in the chemical industry, problems of the structures of cyclosilicates, bulk and thin film polymers, high- $T_{c}$ superconductors, superlattices, and results on initial research and service activities of the Magneto-Raman Spectroscopy Laboratory. The presentations were followed by both formal and informal discussions. These showed that the research program as initially conceived is on the right 
track. Specifically,

1. There is no overlap between the program and others at NIST.

2. The number of other research groups in the USA and elsewhere doing magneto-Raman spectroscopy is small.

3. The focus on high- $T_{C}$ superconducting materials and those with giant magneto resistance should be broadened to include semiconductors, thin films, heterostructures, and others of present and future interest.

4. The present equipment holdings, while adequate for current needs, will have to be expanded to pursue a broader range of problems and serve the needs of the user community.

5. A series of recommendations was made for NIST to assist industry in its analytical and research efforts.

6. A strong recommendation was made to develop a standard reference data base for Raman spectra of polymers. There is an urgent need for such reference data, the lack of which impedes industrial use of the Raman spectroscopic technique in industrial analytical laboratories and on production stream lines.

The workshop was attended not only by NIST staff and the invited speakers, but also by researchers from other agencies. The lists of speakers and attendees are given at the end of this report. 


\section{Table of Contents}

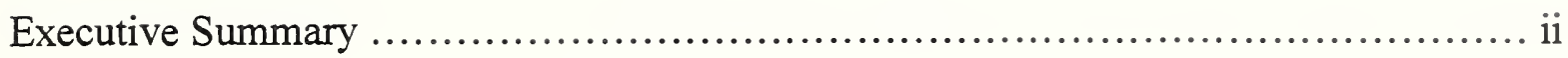

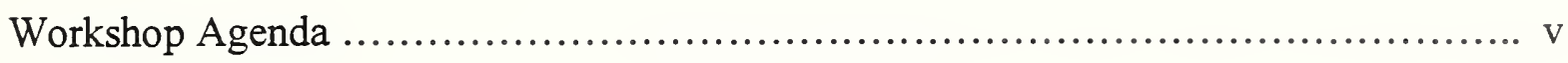

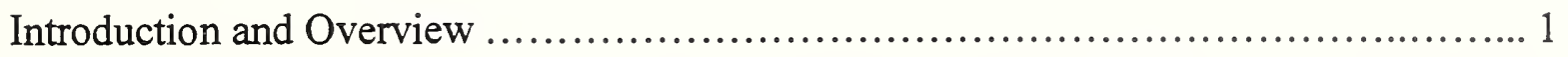

Developments and Applications in Raman Spectroscopy ............................... 3 M. Anne Leugers

Applications of Raman Spectroscopy to Polymer Science and Technology ........ 7 Bruno M. Fanconi

Near-IR FT-Raman Microspectroscopy of Two Materials Systems: CVD

Diamond and Zirconia Ceramics ............................................... 18 Edgar S. Etz

Structure and Spectroscopy of Cyclosilicate Materials

M. I. Bell and D. A. McKeown

Effect of Doping on the Electronic Raman Spectrum of Cuprate

Superconductors

Chris Kendziora

Raman Spectroscopy of Ultrathin Organic Films and Ceramic Precursors 54 John F. Rabolt

Raman Spectroscopy of Semiconductor Heterostructures: Phonons and

Excitons as Probes of Interfaces

Daniel G. Gammon

Magneto-Raman Spectroscopy at NIST: A Raman Laboratory User Facility 76

A. Weber, V.B. Podobedov, J.P. Rice, and D.B. Romero

List of Speakers

List of Attendees 



\title{
Workshop on Raman Spectroscopy in Optical and Materials Scxiences
}

December 10, 1996

\author{
Lecture Room D \\ National Institute of Standards and Technology
}

\section{Agenda}

8:30 - 8:45 Introduction/Opening Remarks

Alfons Weber, Optical technology Division, NIST

8:45 - 9:15 "Developments and Applications in Raman Spectroscopy"

M. Anne Leugers, Dow Chemical Corporation, Midland, MI

9:15 - 9:45 "Applications of Raman Spectroscopy to Polymer Science and Technology"

Bruno Fanconi, Polymers Division, NIST

9:45 - 10:15 "Near-IR FT-Raman Microspectroscopy of Two Materials Systems: CVD Diamond and Zirconia Ceramics"

Edgar S. Etz, Surface and Microanalysis Division, NIST

10:15 - 10:30 Intermission

10:30 - 11:00 "Spectroscopy and Structure of Cyclosilicate Minerals"

Michael I. Bell, Naval Research Laboratory, Washington, DC and

D.A. McKeown, Howard University, Washington, DC

11:00 - 11:30 "Inelastic Light Scattering by Electron Quantum Fluids in Semiconductor Structures"*

Aron Pinczuk, Lucent Technologies/Bell Laboratories

11:30 - 12:00 "Effects of Doping on the Electronic Raman Spectrum of Cuprate Superconductors"

Chritopher A. Kendziora, Naval Research Laboratory, Washington, DC

12:00 - 13:00 Lunch

13:00 - 13:30 "Raman Spectroscopy of Ultrathin Organic Films and Ceramic Precursors"

John F. Rabolt, University of Delaware, Newark, Del. 
13:30 -14:00 "Raman Spectroscopy in Semiconductor Heterostructures" Daniel G. Gammon, Naval Research Laboratory, Washington, DC

14:00 - 14:15 Intermisssion

14:15 - 14:45 "A Raman User Laboratory" Alfons Weber, Optical Technology Division, NIST

14:45 - Group Discussion

*Talk canceled due to illness of speaker. 


\title{
INTRODUCTION AND OVERVIEW
}

\author{
Alfons Weber \\ Optical Technology Division \\ National Institute of Standards and Technology \\ Gaithersburg, MD 20899
}

Raman spectroscopy has come a long way since the days when the mercury arc lamp, the prism spectrograph and the photographic plate were the standard equipment available. In those days the applicability of Raman spectroscopy was limited to molecular structure studies and only rarely and with great difficulty were studies of crystals undertaken.

The invention of the laser in 1960 changed all that. The first public report of a Raman spectrum generated by a laser (a pulsed ruby laser) was probably the talk given by Sergio Porto, then of the Bell Telephone Laboratories, at the famous Symposium on Molecular Spectroscopy at the Ohio State University. His was probably one of the worst Raman spectra that one would dare to show in public and there were many in the audience who grumbled about what is so good about the laser; they would rather stay with their well developed mercury arc lamp technique. Yes indeed. But then, what is so good about a new born baby?

There is no need to belabor the point, but Raman spectroscopy has come to be an important working tool in basic research and technology, with applications ranging from biotechnology to semiconductor physics, to high temperature superconductors, and others. It is no longer restricted to the carefully controlled laboratory environment, but is now found also on the production line for process monitoring and in the field for in situ monitoring of harmful chemical species in ground water, sampling and ultimate destruction of chemical and biological warfare agents, to mention just a few. These and other applications are the result of developments in lasers, spectrometers, fiber optics, detectors and, not to be ignored, the computer. NIST has taken advantage of these developments and there are quite a few research projects and programs that are successfully pursued by means of Raman spectroscopy. The Magneto-Raman Facility in the Optical Technology Division is a new laboratory and it is of interest to look around and take note of the various Raman spectroscopic projects now pursued at NIST/Gaithersburg.

It should come as no surprise that the Chemical Sciences and Technology Laboratory supports the largest number of projects in Raman spectroscopy. Seven distinct efforts are underway in five divisions of this Laboratory. In the Biotechnology Division, Dr. Adolfas Gaigalas of the Bioprocess Engineering Group studies Raman spectra of biomolecules, in particular Surface Enhanced Raman Spectra (SERS) and Surface Enhanced Resonance Raman Spectra (SERRS) of protein molecules on metal electrodes, while in the Biomolecular Materials Group Dr. Anne L. Plant and her coworkers investigate metal supported selfassembled alkanethiol/phospholipid bilayers. In the Process Measurement Division Dr. James E. Maslar of the Reacting Flows Group uses Raman spectroscopy to study the corrosion of metals under conditions of high pressure and temperature, while in the Process Sensing Group Dr. Tonya M. Herne studies self-assembled protein and nucleic acid monolayers for the development of biosensors. In the Microanalysis Research Group of the Surface and Microanalysis Science Division Dr. Edgar S. Etz applies Raman spectroscopy to microchemical analysis of inorganic materials. Dr. Steven J. Choquette of the Chemical Sensing and Automation Technology Group of the Analytical Chemistry Division uses Fourier Transform Raman spectroscopy (FT-Raman) as well as Fourier Transform Infrared Spectroscopy (FTIR) to determine the oxygen content in gasoline.

In the Materials Science and Engineering Laboratory Dr. Linda M. Braun of the Ceramics Division is engaged in the characterization of macroscopic and microstructural stresses in ceramic systems by micro-Raman spectroscopy, 
while Dr. Bruce O. Fowler of the Dental and Medical Materials Group of the Polymer Division studies the infrared and Raman spectra of dental ceramics.

In the Semiconductor Electronics Division of the Electronics and Electrical Engineering Laboratory Dr. Paul M. Amirtharaj uses infrared and Raman spectroscopy for the characterization of semiconductor materials.

The most recently established effort in Raman spectroscopy is that of the Optical Technology Division of the Physics Laboratory. Initiated in mid-1995 but not fully operational until March 1996, work of this laboratory is devoted to research on solid state substances, such as high$\mathrm{T}_{\mathrm{C}}$ superconducting materials, systems exhibiting giant-magneto resistance, and others. At the same time this laboratory is to serve as a user facility for those members of the NIST community who desire Raman spectroscopic data, either because they do not have their own apparatus or, if they do, are in need of data not obtainable with their own equipment. Details about this laboratory facility are presented in the last paper of these proceedings.

The purpose of this Workshop is two fold. In light of the many different Raman spectroscopic activities that are underway at NIST it is desirable to have a forum which facilitates communication between the various groups. A part of this attempt is to inform the NIST community about the new facility available in the Optical Technology Division. The second

goal is to interact with groups outside NIST and gain information about critical issues in chemistry, physics, and engineering that can be addressed by Raman spectroscopy. With these goals in mind speakers from outside as well as within NIST were selected to inform us about ongoing work and future directions to be taken by Raman spectroscopy, and the needs of industry and technology that can be addressed by work conducted at NIST. In addition to the talks presented by the invited speakers, there were brief reports by several NIST staff on their ongoing research in Raman spectroscopy to foster communication within the institution concerning problems of common interest.

These proceedings are the formal expressions of the talks given by the speakers. For the most part they represent topical reviews of their own as well as others' Raman spectroscopic investigations that have been reported in the literature, but there were also some reports of as yet unpublished and ongoing research. In some cases hard copy was received from the speakers. These were then edited for presentation in a common format. In other cases the written record had to be constructed by the editor from the audio-visual tape recordings of the work shop. All authors were consulted and the final copies of their contributions were reviewed by them for concurrence. Any remaining errors are, however, the sole responsibility of the editor.

The editor and contributors hope that the accounts presented in these proceedings will be found useful in providing an overview of research in forefront areas of Raman spectroscopy and serve as a guide for implementing future activities in this field. 


\title{
Developments and Applications in Raman Spectroscopy
}

\author{
M. Anne Leugers \\ Analytical Sciences Laboratory \\ The Dow Chemical Company \\ Midland, Mich.
}

\section{Introduction}

Raman spectroscopy has recently found an increasing number of applications in industrial laboratories. Not only this, but as a result of new developments in instrumentation Raman spectroscopy is gradually making its way as a quality control tool in chemical process stream lines. As a major chemical manufacturer Dow is very much involved in these aspects, and what I will say would not differ much, in general terms, from the situation in other analytical research laboratories in the chemical industry.

Our analytical sciences laboratory addresses many different needs and there is no single technique that can meet all of them. Our scientific/technical staff of approximately 240 people is therefore organized into groups which reflect the various methods of analytical chemistry. At the present time we support groups dealing with separations technology, materials research, surface analysis including microscopy and $x$-ray techniques, inorganic analysis, molecular spectroscopy, environmental analysis, process analytical chemistry, reactive chemistry, and thermal analysis. In spite of this apparent richness of activities there are areas in which we are not active but would appreciate having outside assistance. This, however, will be mentioned at the end of this paper.

Our activities primarily support research groups and manufacturing problems. As part of this effort we engage in the development of new or better methods of analysis, develop and transfer quality control methods and tools for online production as well as for laboratory work. We are also interfacing with researchers from academia and the national laboratories in areas of mutual interest.
In the following I give a brief description of our experimental facilities in the area of molecular spectroscopy. Several examples will also be given of work done in our research laboratory.

\section{Spectroscopic Facilities}

Of the various experimental techniques mentioned above I will concentrate on that of molecular spectroscopy, with emphasis on Raman spectroscopy. The oldest piece of apparatus in our laboratory is a home built fiber optic Raman spectrometer mounted on a movable cart; the excitation source is an $12 \mathrm{~mW}$ $\mathrm{He}-\mathrm{Ne}$ laser. Additional vibrational spectroscopic instrumentation are a Mattson Genesis spectrometer; a Nicolet 750 FTIR with NIR and Raman bench; home built fiber optic spectrometer on a cart with Holoplex and EEV 15-11 detector and a diode pumped YAG laser source; a Nicolet 710 equipped with a Nic-Plan microscope; a Perkin-Elmer Paragon; and a Dilor XY triple spectrometer quipped with a microscope, CCD detector and argon and krypton laser sources. All of these instruments are in use and are applied to the solution of various problems.

\section{Recent Applications}

Among the recent applications, I would like to briefly mention the on-line analysis of monomers in the production of polymers, the quantitation of pharmaceutical polymorphs, online polymer reactant analysis, the unsaturation of oxygenated solvents, the mapping of crystallinity in polymer parts, the phase mapping 
of ceramic green ware, and studies of in situ deformation of polymers.

Our goal in on-line polymer analysis is the quantification of the amount of monomer on solid particles of polymer in a heterogeneous stream. For this purpose we use an apparatus based on a Kaiser Holospec spectrometer with $532 \mathrm{~nm}$ excitation. One of the advantages of this type of work is the ease of off-line calibration of the laser Raman conversion measurements.

The chemistry of polymer formation must be well understood before good quality polymers can be produced reliably, day-in, day-out. We have, therefore, been working on the development of an on-line method for the quantitation of reactive functionalities in viscous, high molecular weight polymers.

Unsaturation in oxygenated solvents is a problem of major proportions. We are therefore engaged in the development of a fast quality control method for the determination of unsaturation in viscous, high molecular weight oxygenated solvents. In this work we use a Kaiser Holospec laser Raman spectrometer equipped with a Nd:YAG laser source. After initially using the Kaiser probe in the experiments we decided to use our own in-house designed probe for the sampling. Using this probe we have been able to detect concentrations as low as 100 ppm unsaturation.

Highly-filled plastic parts often exhibit a degree of crystallinity which affects performance. Tools for the analysis of polymer crystallinity $v s$ depth in molded, filled plastics are therefore being developed. Here the Raman microprobe is successfully used to sample different regions of the polymer specimen.

Green ceramic parts are not homogeneous and we are working on the development of techniques for the mapping of phase of ceramic green ware. Methods which are very similar to those used to map out the crystallinity and depth profile of a polymer are used in these studies to characterize ceramic green ware.

An important aspect of the behavior of plastic parts in commercial devices is the degree of mechanical stability. We therefore undertook in situ studies of the deformation of polymer fibers as they are stressed. In this work we used the Kaiser spectrometer and probe described above with $532 \mathrm{~nm}$ excitation. However, the deformations took place at a time scale so fast, that we could not make dynamic measurements with the Kaiser probe. To get around this problem we had to use new material for each new measurement. We found that we increase the chain length of the side chains on the backbone results in an increase of the degree of crystallinity of the polymers.

\section{Need for Outside Resources}

As described very briefly above, our work is of a varied nature. We do not have all the required means to accomplish our goals and I would just like to give a brief list of studies for which we are seeking outside assistance.

a. There is a need for the mapping of highly fluorescent polymers/beamsensitive matrices.

b. Conventional Raman techniques can only map small areas of a surface, the size depending on the spot diameter of a focused laser beam. More general, twodimensional vibrational imaging is needed to map out the surface properties of a material.

c) Most of the work done in an analytical support laboratory requires only vibrational resolution, i.e., resolution of the order of 1 to $2 \mathrm{~cm}^{-1}$. There are occasions were, however, much higher resolution is needed, particularly in the trace analysis of gases. For such purposes one needs resolution of the order of $0.005 \mathrm{~cm}^{-1}$ or better.

d. Nearly all Raman spectroscopy of materials is done with visible or nearinfrared excitation, the latter method being used to avoid problems due to fluorescence or luminescence of the samples. There is, however, also a need for Raman spectroscopy with ultraviolet excitation. We are at present not equipped to pursue such studies.

e. There is a need for systematic studies of optical materials for in situ Raman analysis. Such studies have not, I believe, been done as yet. There are studies which require pressurized Raman cells, with pressures ranging up to 10,000 psi.

f. We need to develop "quasi-universal" seal designs for optical probes which may be located in hostile environments. 
g. Lastly, I must mention the virtual absence of calibration standards sets for the calibration and evaluation of imaging spectrometers.

This list of items is not necessarily exhaustive. It indicates, however, a range of problems which an industrial chemical analytical laboratory is faced with and which it is not always equipped to solve on its own.

\section{Summary}

In this talk I have given an outline of problems that animate the work of the staff of an industrial analytical laboratory. Even with generous in-house support there are problems that require for their solution the assistance of outside partners and we welcome any interest in such arrangements.

\section{Discussion}

Q Is Raman used in process monitoring and process control?

A We use it right now. An analytical technique is put on line. Operators monitor the signals off a computer. Periodically the analytical results are checked and after some time the results are incorporated into a closed loop control. This is done, however, only after extensive checks to assure that there are no false signals and that the technology used is reliable.

Q What is the typical spectral resolution used in the work?

A In the dispersive systems resolutions down to about $0.5 \mathrm{~cm}^{-1}$ can be obtained but such are rarely used in the Raman work. In the infrared, however, these are needed for gas correlation measurements. If gas phase spectra are analyzed for trace species then even higher resolution is required, of the order of $0.005 \mathrm{~cm}^{-1}$. A high resolution Bomem FTIR spectrometer was previously used but this instrument is no longer available.

Q At the end of your talk you indicated spectroscopic needs that could be met by outside investigators. How important is UV Raman and 2D-imaging?

A This is an exciting area. Previously the only competing technologies were $x$-ray imaging or synchrotron sources. We have a very strong microscopy group but in many cases it is difficult to identify the samples by microscopy only. Microscopy coupled with Raman imaging allows molecular characterization of the sample.

Q What kind of spatial resolution do your applications demand and how does this compare with what you actually see?

A Generally we do not need more than 1 micron in spatial resolution.

Q Would you say that 785 nanometer application would solve $90 \%$ of your problems without luminescence appearing? Or are you forced to go to $1064 \mathrm{~nm}$ ?

A I think that $785 \mathrm{~nm}$ would work probably about $95 \%$ of the time, although there are cases such as in the example of the polymer shown where we are looking at the $\mathrm{C}-\mathrm{H}$ stretch from $785 \mathrm{~nm}$ which is not a good place to work because the response is falling off pretty badly by then so that I would really like to use the $1064 \mathrm{~nm}$ line, but I am not comfortable with on-line FT-Raman systems yet. 
Q Because of the detector fall off beyond $3200 \mathrm{~cm}^{-1}$ ?

A No, but just because of the mechanical reliability. I don't know that any of the vendors are really emphasizing FT-Raman for process applications. For our on-line FTIR applications we test all of our systems for reliability before putting them on-line.

Q Is the Raman signature obtained from the stressed fiber reversible when the fiber is relaxed?

A In the high density material the signal is reversible but in low density materials there is evidence of some crystallinity developing as the result of one stretch. 


\title{
Applications of Raman Spectroscopy to Polymer Science and Technology
}

\author{
Bruno M. Fanconi \\ Polymers Division \\ National Institute of Standards and Technology \\ Gaithersburg, MD 20899
}

\section{INTRODUCTION}

As a vibrational spectroscopy, Raman scattering is capable of elucidating the chemical and physical properties of materials, including polymers. In practice, however, infrared spectroscopy is by far the preferred method, particularly for routine analysis. In part, this preference derives from the near exclusive use of infrared prior to the commercialization of $\mathrm{CW}$ gas lasers. As a result, rich sources of infrared reference data are available for identification of unknowns - for example, computer searchable data bases that contain tens of thousands reference spectra, and infrared extinction coefficients for a large number of molecules and chemical moieties. Furthermore, infrared instrumentation, including FTIR, are compact, user friendly and relatively inexpensive.

When laser excitation sources for Raman spectroscopy became commercially available in the early 70s many of us thought that Raman spectroscopy would evolve to the point that it would compete with infrared spectroscopy in characterization of polymeric materials. About the same time, however, improvements in dedicated computers for instrumentation led to commercialization of FTIR. Even with adapting interferometric techniques into Raman, FTRaman, it is unlikely that this inelastic scattering technique will ever reach the importance of infrared in polymer characterization.

The big killer for Raman spectroscopy in polymer characterization is fluorescence. Even for polymers whose basic molecular constituents do not fluoresce at frequencies of the $\mathrm{Ar}$ ion laser, fluorescence is often encountered. This derives from adventitious impurities, such as remnants of catalysts, additive impurities, and oxidation products. Frequently, it is possible to remove these impurities, either through purification or by photobleaching. An advantage of FT is to allow the use of longer wavelength excitation, thereby circumventing the absorption that leads to luminescence.

Let me add that although most of the following material will be limited to synthetic polymers much of it will apply to biological polymers, as well.

What then is the measurement role for Raman spectroscopy in polymers? The following list captures what I believe to be the essential advantages.

1) Vibrational modes, undetectable by ir, that reveal unusual features:

Complementary data for normal coordinate analysis;

Longitudinal and transverse acoustic

modes;

Frequency shifts under mechanical load;

Molecular orientation in polymer melts

and shear,

Stress distribution,

Creep,

Instantaneous Young's modulus,

Probes of stress concentration.

2) Remote sensing - an example is the use of optical fibers to direct light into molds and other polymer processing equipment where measurements can be made remotely, such as monitoring the cure of thermosets.

3) Spatial resolution - the short wavelength of Raman excitation gives advantages over infrared in spatial resolution, incorporation of near field optical techniques and AFM (atomic force microscopy) - NSOM (near field scanning optical microscopy) may well lead to spatial resolution in the submicrometer range.

4) Ease of sampling - as long as light can be delivered to and recovered from a sample a Raman spectrum may be obtained, in principle.

5) Vibrational spectroscopy in aqueous solution - although much more important 
in biopolymers, the weak Raman scattering from water throughout the midinfrared permits examination of polymer structure in aqueous media where infrared is virtually useless.

Examples are given in each of the first three mentioned areas. Ease of sampling is a distinct advantage in most of the applications that are described.

\section{Normal Coordinate Analysis}

Since Raman data frequently complement infrared it has been useful in refining force fields in polymers. An example is polyethylene, the lattice structure of which has $D_{2 h}$ symmetry and the spectroscopically active modes are observed in either infrared or Raman, but not both. Table I lists the experimental and calculated frequencies

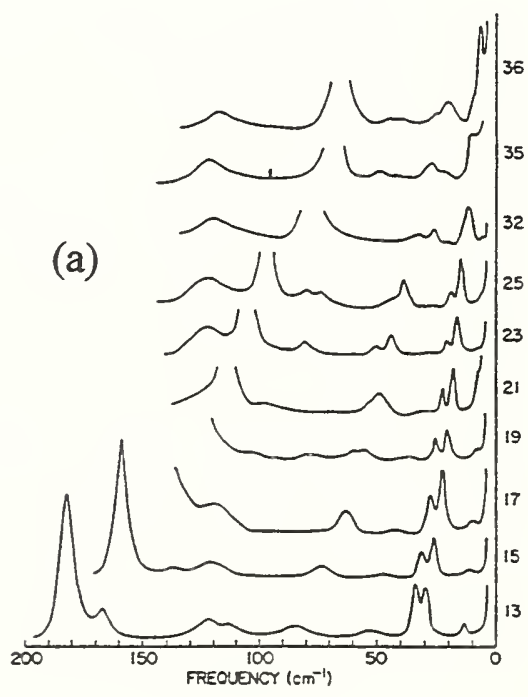

physical structure, particularly over large distances. Fortunately, long wave modes, primarily with frequencies below $100 \mathrm{~cm}^{-1}$, are observable by Raman, and furthermore the low frequency region is accessible experimentally. Fig. $1 \mathrm{~b}$ gives the normal mode frequencies of polyethylene as function of the phase, or wave vector. The curves were calculated using the force field that yielded the calculated frequencies shown in Table I. The data points at phase $=0$ are Raman observed and those at $\pi$ are infrared active. All other modes are spectroscopically inactive in the ideal polyethylene lattice, but are active in the normal alkanes which are isostructural with polyethylene, except for their finite chain length. To each of the observed frequencies [2] in the n-alkanes, Fig. la, can be assigned a corresponding mode, and this assignment yields the rest of the experimental points.

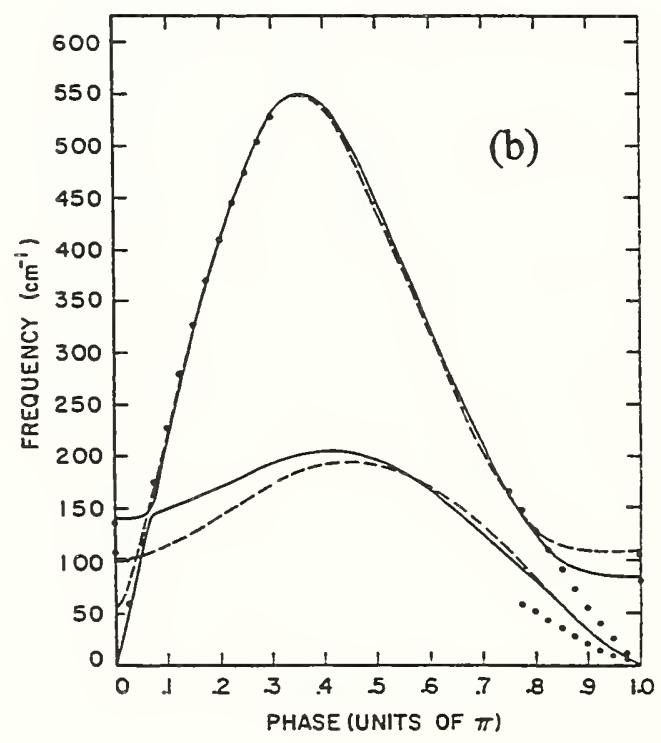

Fig. 1. Skeletal vibrations of polymers: longitudinal and transverse modes.

for polyethylene and their assignments to the normal modes of vibration [1]. Potential energy functions derived from vibrational analysis are used in calculations of polymer structure, both in the solid and liquid state.

\section{Longitudinal and Transverse Acoustic Modes}

The long chain structure of polymers gives these materials their distinctive properties, hence there is interest in measurements of chemical and
In particular, let us focus on the frequencies at low phase values. These correspond to the intense band seen in the alkane spectra, and are assigned to longitudinal acoustic modes (LAM). Figure 2 shows more n-alkane [3] data and that the frequency of the corresponding mode approaches zero as the length of the n-alkane increases; the lowest frequency comes from an $\mathrm{n}$-alkane 94 carbons in length, or about $12 \mathrm{~nm}$ in length. It is rather extraordinary to observe vibrational frequencies sensitive to structure over distances in tens of nanometers. 


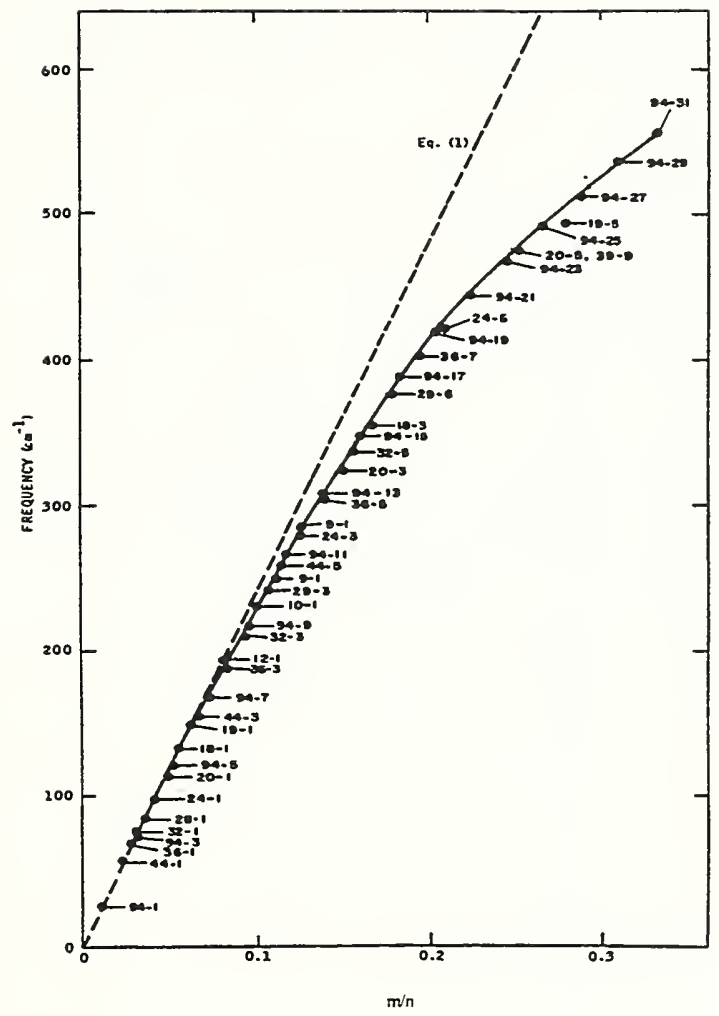

Fig. 2. Longitudinal acoustic modes in n-alkanes, after reference [3].

More striking is the observation of LAM in polymers that are hundreds of nanometers in length. This is because semicrystalline polymers form lamellar structures tens of nanometers in thickness, shown in the bottom part of Fig. 3 for polyethylene crystals grown from solution.
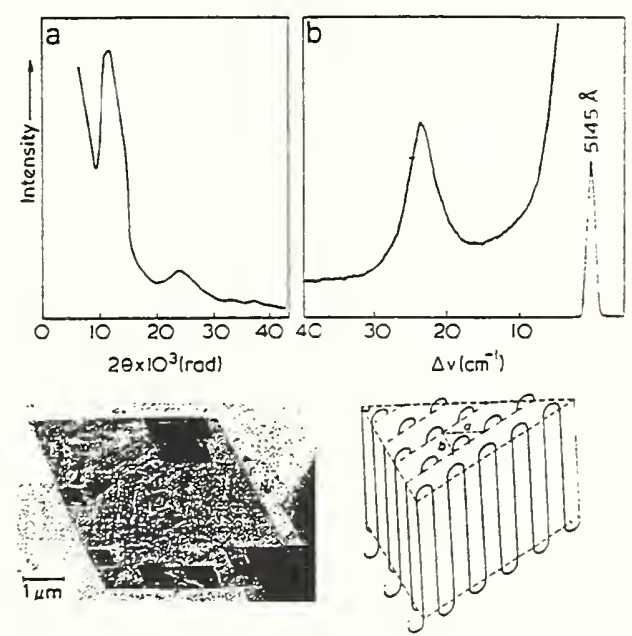

Fig. 3. Small angle $x$-ray diffraction (SAXD) pattern (a) and Raman spectrum (b) of polyethylene single crystals grown at $85^{\circ} \mathrm{C}$. Figures labelled a and $\mathrm{b}$ are taken from reference 4 .
The tortuous chain path through the crystal is shown at the right. The thickness of the lamella, an important morphological feature, may be detectible by small angle $x$-ray diffraction (SAXD). A SAXD peak is observed when the lamellae are stacked one on top of the other and the diffraction peak corresponds to the lamellar thickness, including the fold regions. From the n-alkane plot, shown in Fig. 2, a length can be assigned to the Raman band. This is shown in Fig. 4 where $\mathrm{x}$-ray and Raman data [5] are

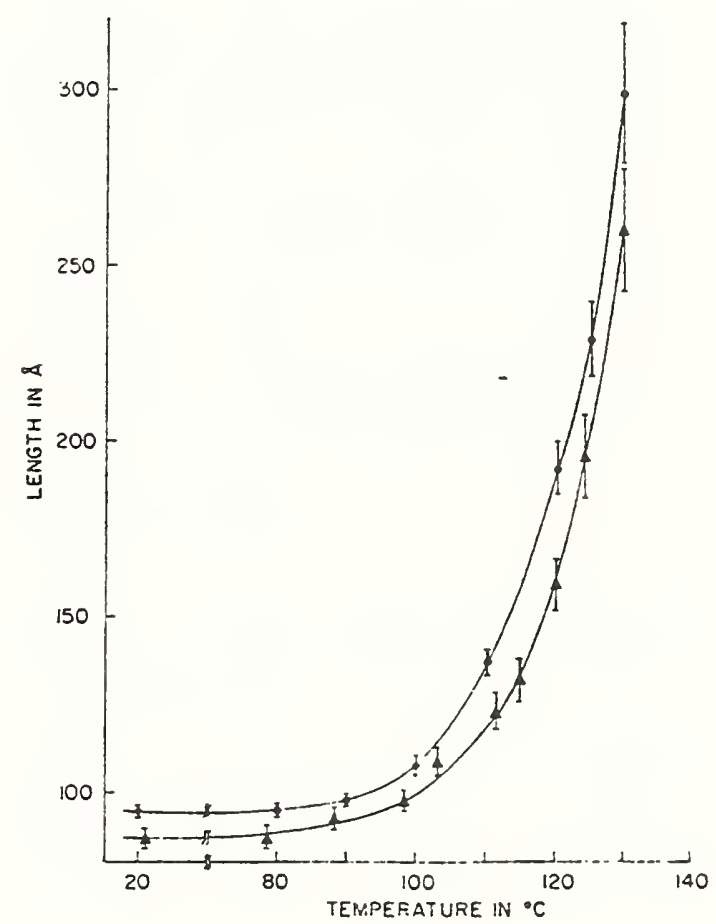

Fig. 4. Dependence of fold length of polyethylene single crystals on annealing temperature - are $\mathrm{x}$-ray long spacing and $\Delta$ are predicted from low frequency Raman spectra.

compared for polyethylene single crystals that have been annealed, a process known to increase lamellar thickness. A simple interpretation is that the difference between these two curves represents the thickness of the fold region.

Because the Raman LAM band derives from individual chains, rather than stacks of lamellae, it has been used to deduce morphology in melt crystallized polyethylene. The three methods (SAXD, Raman and electron microscopy) show results consistent with a single population of lamella, when the sample is quenched from the melt. By contrast, the Raman spectrum of an 
TABLE I. Experimental and calculated frequencies of polyethylene (orthorhombic structure)

\begin{tabular}{|c|c|c|c|}
\hline Symmetry & Calc. $\left(\mathrm{cm}^{-1}\right)$ & Obs. $\left(\mathrm{cm}^{-1}\right)$ & Assignment \\
\hline$A_{g}$ & \multicolumn{3}{|c|}{ Raman active } \\
\hline & $\begin{array}{r}141.2 \\
1137.5 \\
1175.2 \\
1445.0 \\
2854.6 \\
2884.8 \\
\end{array}$ & $\begin{array}{r}136 \\
1133 \\
1170 \\
1442 \\
2848 \\
2883 \\
\end{array}$ & $\begin{array}{l}\text { libration } \\
\text { optical skeletal } \\
\text { methylene rock } \\
\text { methylene scissors } \\
\text { symmetric C-H stretch } \\
\text { asymmetric C-H stretch }\end{array}$ \\
\hline \multicolumn{4}{|l|}{$A_{u}$} \\
\hline & $\begin{array}{r}54.9 \\
1040.6 \\
1180.8\end{array}$ & $\begin{array}{l}\text { in active } \\
\text { inactive } \\
\text { inactive }\end{array}$ & $\begin{array}{l}\text { translatory } \\
\text { methylene twist } \\
\text { methylene wag }\end{array}$ \\
\hline$B_{1 g}$ & \multicolumn{3}{|c|}{ Raman active } \\
\hline & $\begin{array}{l}1065.8 \\
1296.8 \\
1370.7\end{array}$ & $\begin{array}{l}1065 \\
1297 \\
1370\end{array}$ & $\begin{array}{l}\text { optical skeletal } \\
\text { methylene twist } \\
\text { methylene wag }\end{array}$ \\
\hline \multirow[t]{2}{*}{$\mathrm{B}_{\mathrm{lu}}$} & \multicolumn{3}{|c|}{ Infrared active } \\
\hline & $\begin{array}{r}0.0 \\
85.9 \\
736.5 \\
1477.8 \\
2851.5 \\
2921.5\end{array}$ & $\begin{array}{r}81 \\
734 \\
1473 \\
2851 \\
2919\end{array}$ & $\begin{array}{l}\text { translatory } \\
\text { methylene rock } \\
\text { methylene scissors } \\
\text { symmetric C-H stretch } \\
\text { asymmetric C-H stretch }\end{array}$ \\
\hline \multirow[t]{2}{*}{$\mathrm{B}_{2 \mathrm{~g}}$} & \multicolumn{3}{|c|}{ Raman active } \\
\hline & $\begin{array}{l}1069.6 \\
1293.4 \\
1373.2\end{array}$ & $\begin{array}{l}1068 \\
1295 \\
1370\end{array}$ & $\begin{array}{l}\text { optical skeletal } \\
\text { methylene twist } \\
\text { methylene wag }\end{array}$ \\
\hline \multirow[t]{2}{*}{$\mathrm{B}_{2 u}$} & \multicolumn{3}{|c|}{ Infrared active } \\
\hline & $\begin{array}{r}0.0 \\
109.1 \\
720.7 \\
1472.3 \\
2852.9 \\
2921.7\end{array}$ & $\begin{array}{r}106 \\
721 \\
1463 \\
2851 \\
2919\end{array}$ & $\begin{array}{l}\text { translatory } \\
\text { methylene rock } \\
\text { methylene scissors } \\
\text { symmetric } \mathrm{C}-\mathrm{H} \text { stretch } \\
\text { asymmetric } \mathrm{C}-\mathrm{H} \text { strclch }\end{array}$ \\
\hline \multirow[t]{2}{*}{$\mathrm{B}_{3 \mathrm{~g}}$} & \multicolumn{3}{|c|}{ Raman active } \\
\hline & $\begin{array}{r}101.5 \\
1137.0 \\
1176.1 \\
1458.3 \\
2849.9 \\
2888.7\end{array}$ & $\begin{array}{r}108 \\
1133 \\
1170 \\
1442 \\
2848 \\
2883\end{array}$ & $\begin{array}{l}\text { libration } \\
\text { optical skcletal } \\
\text { methylene rock } \\
\text { methylene scissors } \\
\text { symmetric C-H stretch } \\
\text { asymmetric } \mathrm{C}-\mathrm{H} \text { strctch }\end{array}$ \\
\hline \multirow[t]{2}{*}{$\mathrm{B}_{3 \mathrm{u}}$} & \multicolumn{3}{|c|}{ Infrared active } \\
\hline & $\begin{array}{r}0.0 \\
1042.9 \\
1177.3\end{array}$ & $\begin{array}{l}1050 \\
1175\end{array}$ & $\begin{array}{l}\text { methylene twist } \\
\text { methylene wag }\end{array}$ \\
\hline
\end{tabular}


isothermally crystallized sample exhibits two peaks characteristic of a bimodal lamellar texture and this is supported by the electron micrographs [4]. The SAXD, on the other hand, reveals only a single peak corresponding to the lamellar population formed at the isothermal crystallization temperature. These then are examples of how Raman spectroscopy can be used to deduce morphological features in the size realm of electron microscopy.

Because LAMs are acoustic modes, from knowledge of length by SAXD the LAM Raman frequency may be used to estimate the Young's modulus (see Fig. 5) through the elastic rod model for longitudinal modes:

$$
v=1 / 2 c L(E / \rho)^{1 / 2}
$$

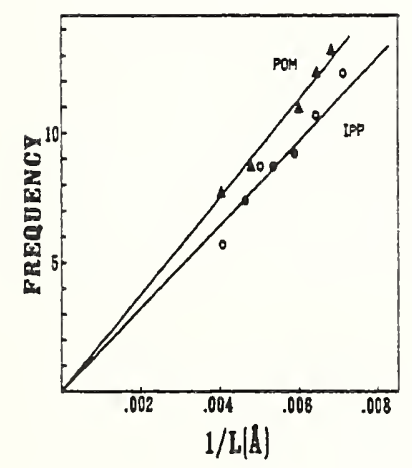

LAM frequencies of polyoxymethylene, POM, and isotactic polypropylene, IPP, vs. reciprocal of long spacing as measured by SAXS.

\begin{tabular}{|c|c|c|c|c|c|}
\hline \multirow[b]{2}{*}{ Polymer } & \multirow{2}{*}{ Conformation } & \multicolumn{3}{|c|}{$\begin{array}{l}\left.\text { e-axis Young's Modulus (dvres/ } / \mathrm{cm}^{2}\right) \\
\text { Nevtron }\end{array}$} & \multirow{2}{*}{ 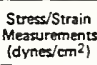 } \\
\hline & & $x$ ray & $\begin{array}{l}\text { Neutron } \\
\text { Scattering }\end{array}$ & Raman & \\
\hline Polventhlene & Planar Zig Zag & $24.0 \times 10^{11}$ & $32.9 \times 10^{11}$ & $35.8 \times 10^{11}$ & $28.8 \times 10^{11}$ \\
\hline $\begin{array}{l}\text { Polvterafrafuoro- } \\
\text { ethyiene }\end{array}$ & $15 / 7$ Helix & $15.6 \times 10^{11}$ & $22.2 \times 10^{11}$ & $20.3 \times 10^{11}$ & - \\
\hline $\begin{array}{l}\text { Polyoxy- } \\
\text { methylene }\end{array}$ & 9/5 Helix & $5.4 \times 10^{15}$ & $96.4 \times 10^{11}$ & $18.9 \times 10^{11}$ & $\begin{array}{l}10.5 \times 1011 \\
12.0 \times 10^{11}\end{array}$ \\
\hline $\begin{array}{c}\text { Iseveceic } \\
\text { Polveropylens }\end{array}$ & 3/1 Helix & $35 \times 1011$ & - & $8.8 \times 10^{11}$ & $3.6 \times 10^{11}$ \\
\hline $\begin{array}{l}\text { Polvethyteng- } \\
\text { oxice }\end{array}$ & $7 / 2$ Helix & $1.0 \times 10^{115}$ & - & $2.2 \times 10^{11}$ & - \\
\hline
\end{tabular}

Fig. 5. Young's modulus determined by Raman spectroscopy. The figure showing the LAM frequencies of POM is taken from reference [6].

In this simple relationship, the LAM frequency is related to the length, $\mathrm{L}$, and the modulus, $\mathrm{E}$. Rho is the density and $c$ the velocity of light. Young's moduli determined from Raman spectroscopic data are shown in Fig. 5. The authors of references [6,7] pointed out deficiencies in the $\mathrm{x}$-ray method of estimating moduli and challenged polymer technologists to develop methods to produce materials with superior mechanical properties.

\section{Frequency Shifts under Mechanical Load}

An advantage of Raman spectroscopy is the ability to make measurements in situations where light transmission studies are difficult, an example being polymer processing equipment. Fig. 6 shows Raman spectra of polyethylene melt under shear in which bands appear at high

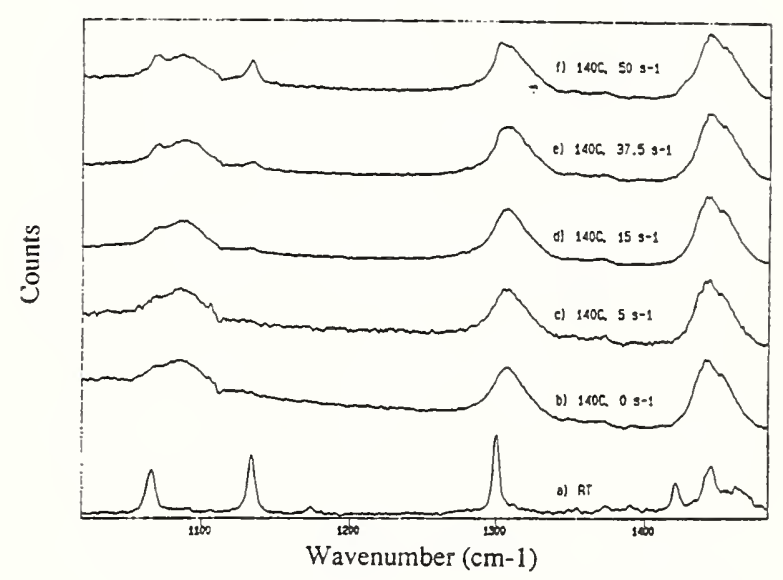

Fig. 6. Rheo-Raman studies of polyethylene melts, after reference [8].

shear indicative of the polyethylene chain stretched out and aligned in the direction of deformation [8].

Raman spectroscopy has also been used to characterize solid polymers under mechanical load. We have fairly detailed knowledge of the physical structure of polymers, and certainly available tools for characterizing structure for those cases where structure is unknown. We also know something of how structure changes on application of mechanical stress, for example a randomly oriented specimen will elongate with polymer chains aligning in the direction of the load. Much less is known about the process by which this occurs. Both Raman and infrared spectroscopy have been used to elucidate these processes [9]. If you think of a polymer chain tugged at either end, like the rope in a tug-ofwar, one imagines that the carbon-carbon bonds that form the polymer backbone become distorted and that this distortion would affect the vibrational frequencies. Since the view is that principally main chain carbon-carbon bonds are affected by stress, Raman spectroscopy would be particularly useful owing to the greater Raman scattering efficiency of these modes as opposed to their weak infrared absorption probabilities. 
The Raman spectra shown in Fig. 7 were from a polyethylene film taken at low temperatures to exclude creep [10]. The polymer chains are
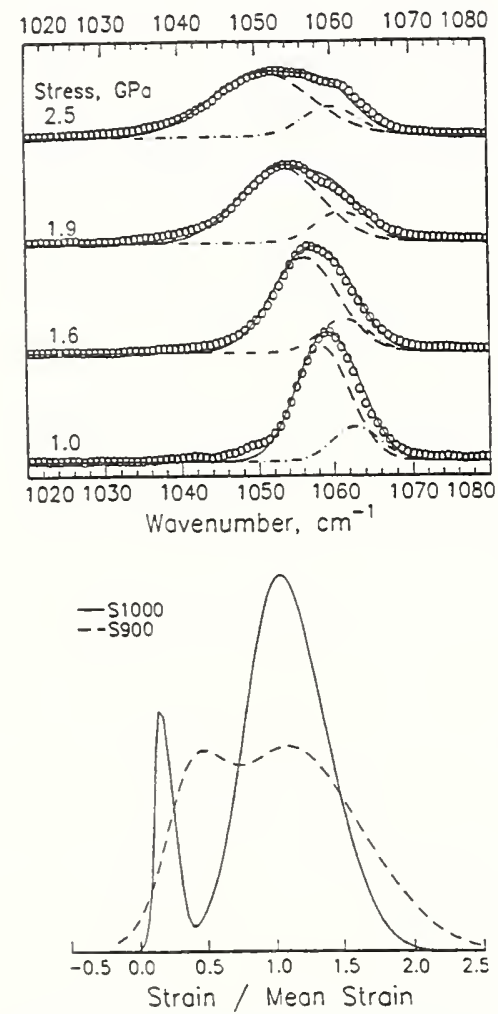

Fig. 7. Stress distribution in polyethylene fibers, after reference [10].

highly oriented in the fiber direction. The Raman band, which is a single band un-stressed, is deconvoluted into two bands, interpreted as representing two different populations of stressed molecules. The frequency maxima of the two bands decrease by different amounts with increasing stress. By ratioing the areas of the two peaks one derives a view of how the stress is distributed between the two populations as the sample is strained. The distributions from two different fibers are compared in the lower part of Fig. 7. The bimodal distribution of stressed chains is consistent with morphological features that evolve in the spinning of: these fibers from gels.

Frequency shifts with stress and strain are also observed in polydiacetylenes [11] (see Fig. 8). These and related highly conjugated macromolecules whose Raman scattering is enhanced through resonance, have been used as probes of mechanical stress in other polymeric materials. Note that a strain of $1 \%$ produces a shift of about 20 wave numbers in polydiacetylene fibers as

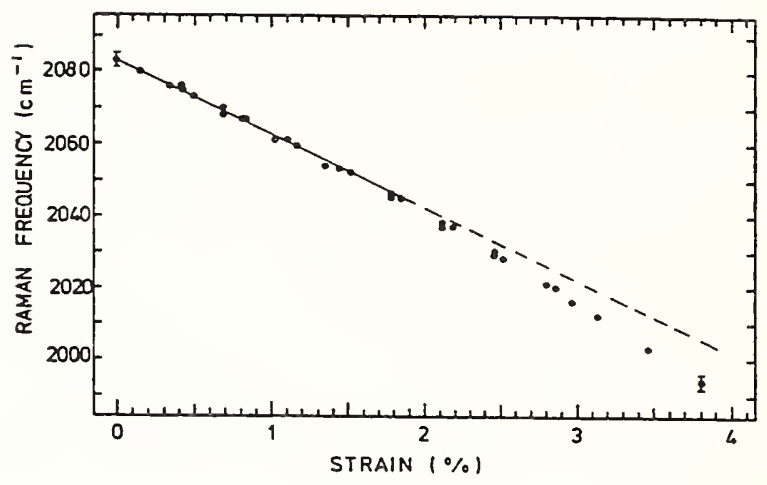

Frequency of the carbon-carbon triple bond stretch of polyacetylene as a function of strain. The solid line is a least-squares fit to the data below $2 \%$ strain.

Fig. 8. Raman measurement of strain, after reference [11].

seen in Fig. 9 [12]. When a similar molecular unit is incorporated into a urethane copolymer Raman spectroscopy may be used to probe the strain at the molecular level in an isotropic material. Figs. 9 and 10 show how the band shifts upon

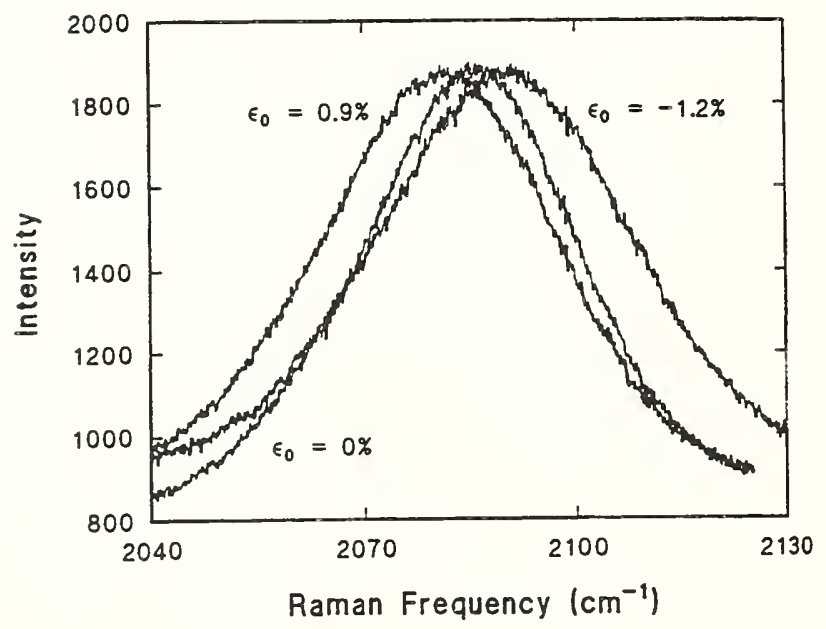

Variation of the carbon-carbon triple bond stretch Rainan band for diacetylene-containing urethane copolymer, undeformed and subject to tensile (positive) and compressive (negative) deformation.

Fig. 9. Raman measurement of local strain, after reference [12].

extension and compression, the latter producing an upwards frequency shift. A strain of $1 \%$ produces a much smaller shift in the urethane 
copolymer revealing a strain distribution at the molecular level, compared to macroscopic strain.

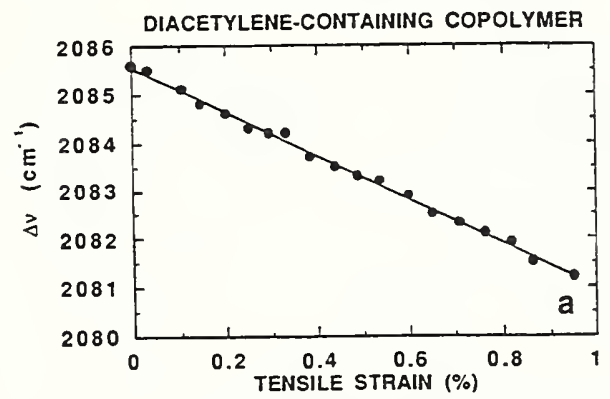

DIACETYLENE-CONTAINING COPOLYMER

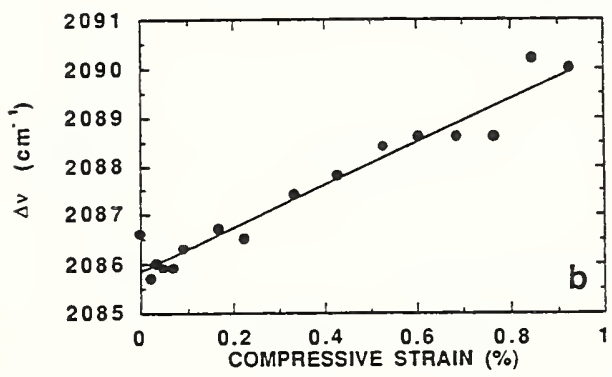

Dependence of peak frequencies of the carbon-carbon triple bond stretching mode Raman band for the diacetyleme-containing urethane copolymer upon: (a) tensile strain and $(b)$ compressive strain.

Fig. 10. Raman measurements of local strain, after reference [12].

\section{Remote Sensing}

Competitive demands are moving polymer processors to adapt sensors that yield information about the material state other than temperature and applied pressure. Here, spectroscopic techniques come into consideration, particularly vibrational spectroscopic techniques that provide information about chemical and physical attributes. Infrared spectroscopy, for the reasons alluded to earlier, is preferable, but transmitting light into generally line-of-sight inaccessible locations, as in molds or extruders, is virtually impossible and ir transmitting fibers suffer from poor transmission, fragility, toxicity and high cost. Here, Raman spectroscopy has a definite advantage in that visible, or at worst, near infrared light is employed and ordinary optical fibers, made of silicates, can be used (see Fig. 11). Problems alluded to concerning fluorescence have to be overcome, however.

An example is the monitoring of the cure of an epoxy resin, in which chemical reactions transform a viscous resin and hardener mixture into a glassy network polymer. Furthermore, knowledge of the state of cure (that is the degree to which chemical reactions have proceeded to completion) is important technologically, since processing decisions (consolidation, removal)are dictated by the cure state. The top part of Fig. 12 shows the epoxy spectrum prior to cure, partially cured and near completion of the reaction [14]. The lower part of the figure shows the cure curve. Data of this type have been integrated into process control routines that adjusts temperature to either accelerate or slow down the reactions, depending on the cure state.

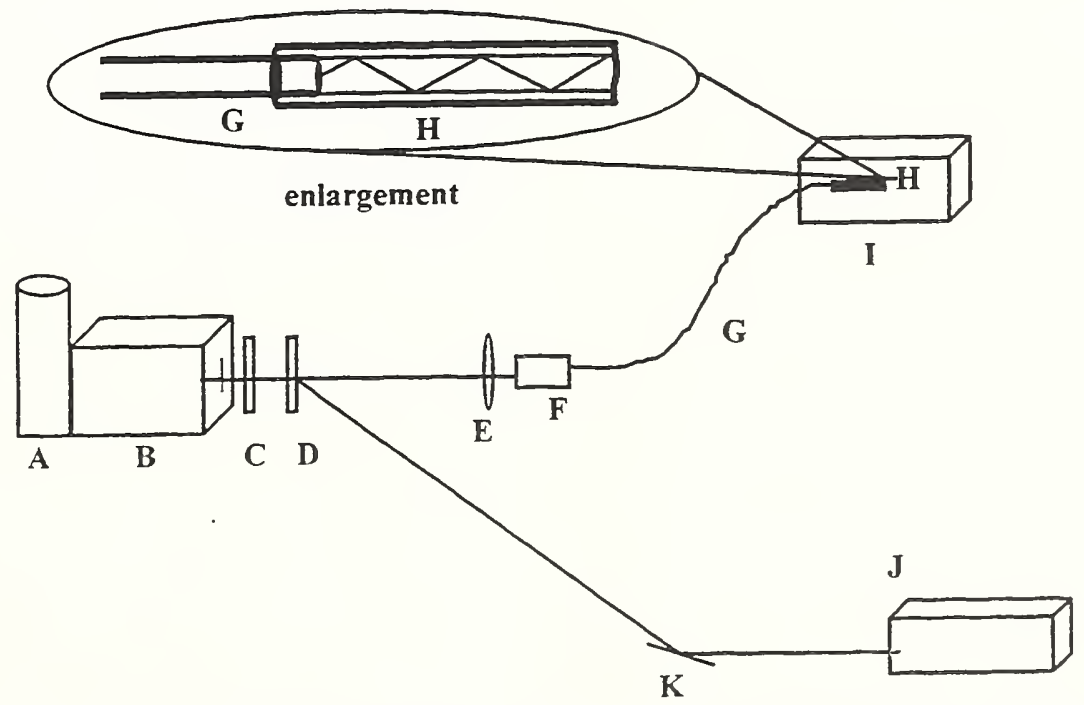

Fig. 11. Schematic diagram of a Raman spectroscopic probe with fiber optics, after reference [13]. 


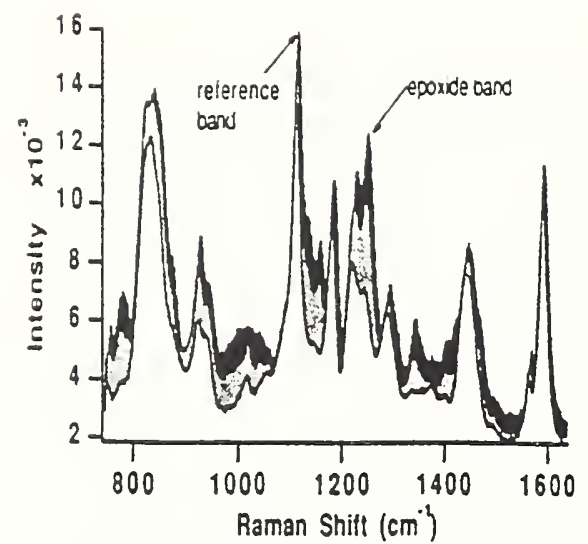

Series of corrected dual-fiber probe, Raman spectra of cpoxy taken: immediately after mixing (black), after 1.2 hours at $75^{\circ} \mathrm{C}$ (gray) and after 2.2 hours at $75^{\circ} \mathrm{C}$ with 1 hour post-cure at $90^{\circ} \mathrm{C}$ (white).

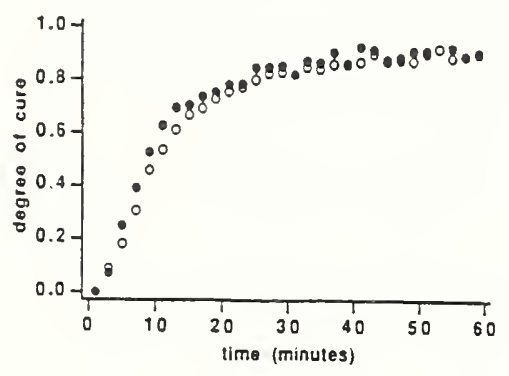

Isothermal degree of cure for epoxy at $90^{\circ} \mathrm{C}$ versus time. Open and filled circles are data taken with different excitation sources.

Fig. 12. Cure monitoring by Raman spectroscopy, after reference [14].

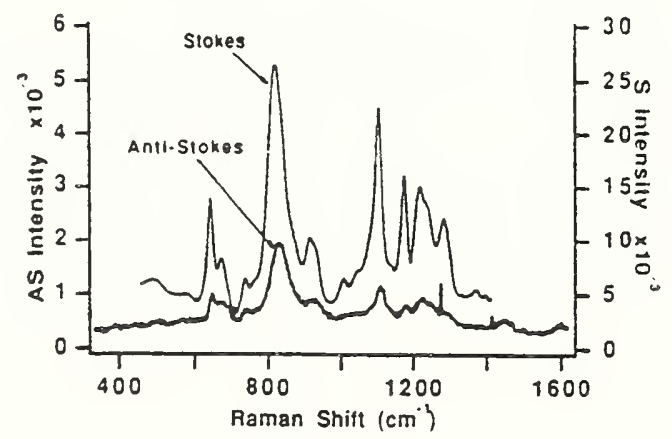

Anti-Stokes and Stokes Raman spectra of cured epoxy at $100^{\circ} \mathrm{C}$.

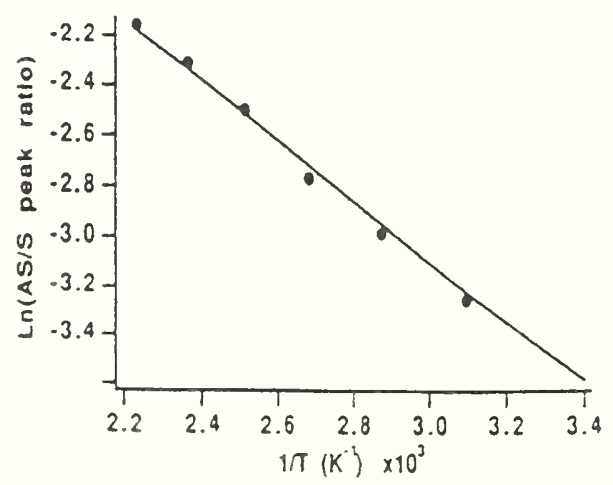

Fig. 13. Measurement of temperature by Raman spectroscopy, after reference [14].
Temperature is one of controlled variables in most polymer processing methods. Owing to viscous heating and exothermic chemical reactions the resin temperature may be quite different from that of the processing equipment, where the temperature measurement is usually made. Owing to the Boltzmann effect Raman spectroscopy can be used as a temperature gauge by ratioing anti-Stokes and Stokes Raman intensities. This is shown for an epoxy resin in Fig. 13 [14]. Again, with optical fibers this measurement could be made through ports normally set aside for temperature or pressure measurements.

\section{Spatial Resolution and Depth Profiling}

A final set of examples covers the use of Raman spectroscopy as a chemical microscopic method. A number of technologies use thin polymer films; microelectronic packaging is an example. But this technology extends into other areas, as well. Even barrier films are composites of several different layers of different types of polymers, with each polymer film being a few micrometers in thickness. When one has a cross sectional view, conventional microscopy may be sufficient as shown in Fig. 14 [15]. An optical fiber

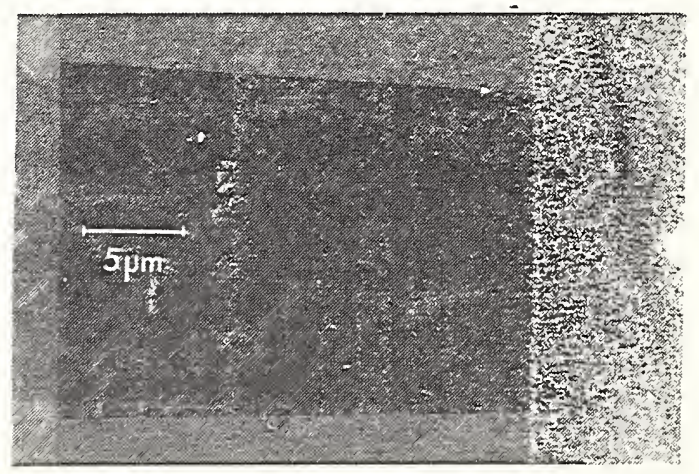

Fig. 14. Optical micrograph of multilayer polymer film assemblies, after reference [15].

technique was used to characterize of polyethylene terephthalate film, one side of which had a higher crystallinity content than the other as shown in Fig. 15.

Higher resolution in the depth mode is possible through confocal microscopy and this method has been use to characterize thin film 


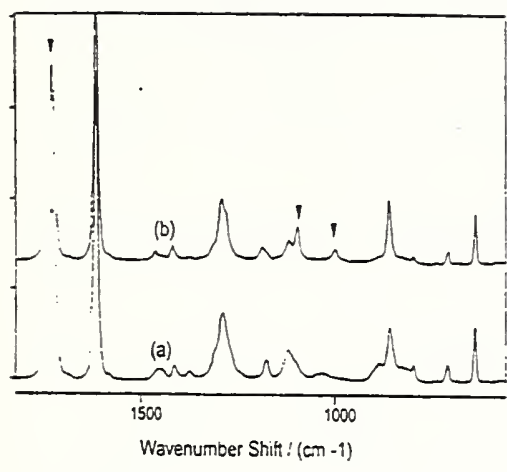

Comparison of Raman spectra of (a) amorphous and (b) semicrystalline films. The spectral features most sensitive to crystallinity clianges are highlighted.

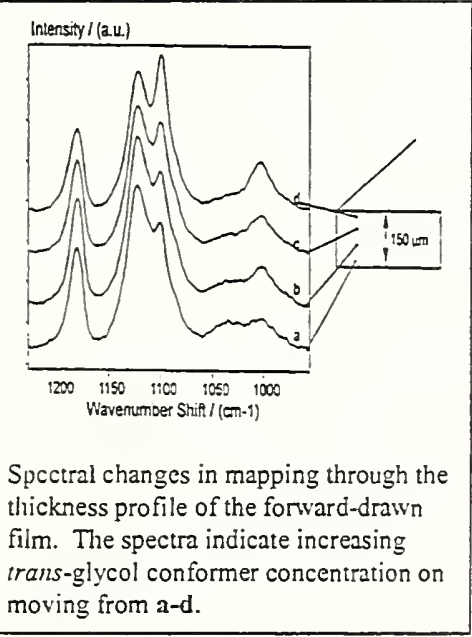

Fig. 15. Depth profiling of polymer films, after reference [16].

assemblies. Fig. 16 describes the principle of confocal Raman microspectroscopy [17]. Fig. 17 shows an example [17]. A 2 micrometer thick polyethylene film was positioned on a thick polypropylene sheet. By optical interference methods it was deduced that a thin layer of air was present between the two. These two figures, $\mathrm{A}$ and $\mathrm{B}$, compare measurements as a function of depth of focus, with and without the pinhole feature of confocal microscopy. With a 300 micrometer pinhole the polyethylene spectrum can be clearly differentiated from the polypropylene. Confocal Raman microscopy studies on biological materials conclude that depth resolution approaching one micron is possible.

Fig. 18 gives another example, one that is relevant to characterizing fiber interfaces in composite materials, an issue of technological importance [17]. Here confocal Raman-micro spectroscopy was use to acquire a spectrum of a
20 micrometer polyethylene fiber embedded in an epoxy matrix.

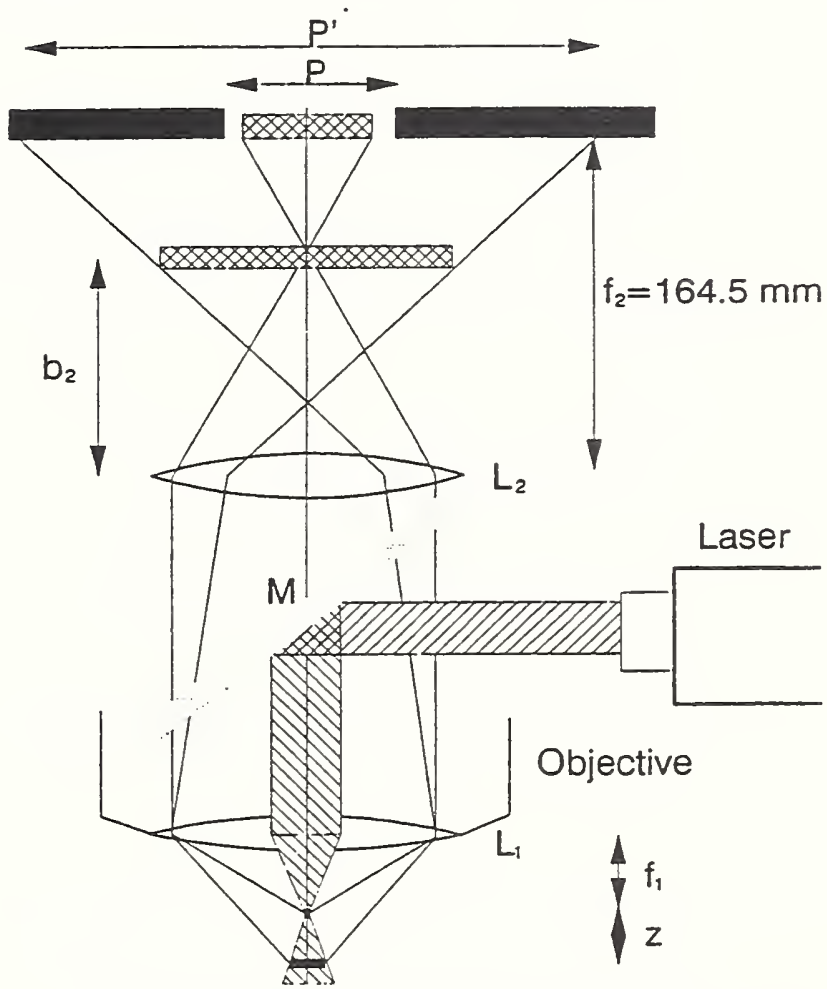

The principle of confocal Raman microspectroscopy. A laser spot in the focal plane passes through the pinhole $P$. A laser spot at a distance $z$ from the focal plane is projected in the image plane with size $P^{\prime \prime}$, and is largely blocked by the pinhole $P$. $L=$ lens; $M=$ beamsplitter; $f_{1}, f_{2}=$ focal length of lens $L_{1}$ and $L_{2}$, respectively; $b_{2}=$ image distance of out-of-focus laser spot.

Fig. 16. Confocal Raman microspectroscopy, after reference [17].
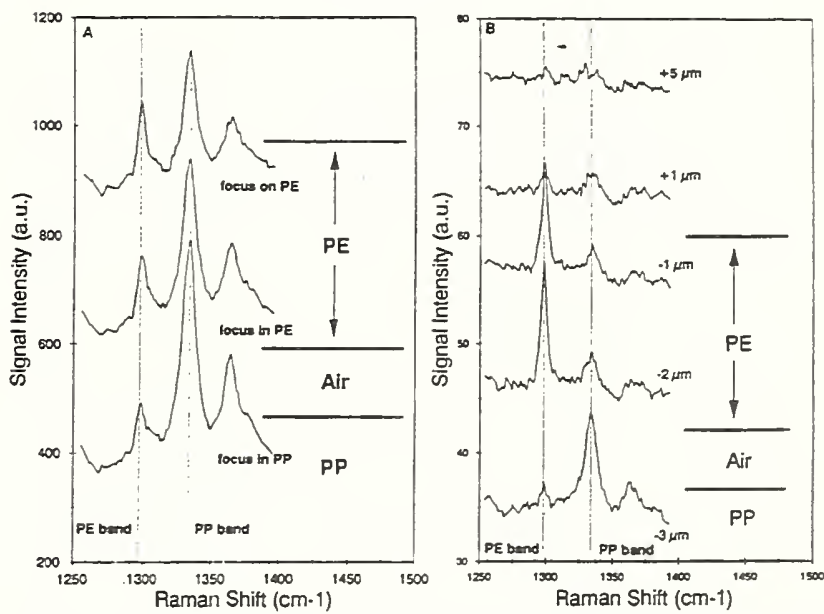

(A) Raman spectrum of a scan through PE/PP system, obtained with the $x 100$ objcctive but without pinhole. (B) Scan through the same system as (A) with the $\times 100$ objective and a $300 \mu$ pinhole. The depths are given with the upper surface of the PE as zero.

Fig. 17. Depth profiling by Raman spectroscopy, after reference [17]. 
Combining Raman spectroscopy with other microscopies, such as scanning near field optical microscopy is likely to lead to even greater improvements in spatial resolution.

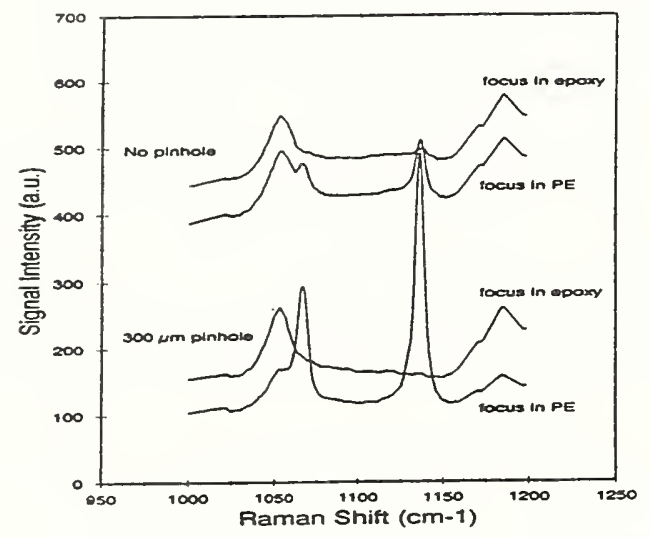

Fig. 18. Raman spectra of a $20 \mu \mathrm{m}$ diameter PE fiber in a $100 \mu \mathrm{m}$ thick epoxy layer, after reference [17].

\section{Conclusions}

In summary, Raman spectroscopy has proven useful in characterizing aspects of polymeric materials not amenable to investigation by infrared techniques. The availability of extensive Raman spectral bases would lead to greater utilization of Raman spectroscopy in the future. IR data are available but hardly any Raman data. This lack of Raman data prevents a more wide spread use of the Raman technique.

\section{References}

1. J. Barnes and B. Fanconi, J. Phys. Chem. Ref. Data 7, 1309 (1078).

2. H.G. Olf and B. Fanconi, J. Chem. Phys. 59, 534 (1973).
3. R.F. Schaufele and T. Shimanouchi, J. Chem. Phys. 47, 3605 (1967).

4. J. Dlugosz, G.V. Fraser, D. Grubb, A. Keller, J.A. Odell, and P.L. Goggin, Polymer 17, 471 (1976).

5. W.L. Peticolas, G.W. Hibler. J.L. Lippert, A. Peterlin, and H. Olf, Appl. Phys. Lett. 18, 87 (1971).

6. J.F. Rabolt and B. Fanconi, J. Polym. Sci. Polym. Lett. Ed. 15, 121 (1977).

7. B. Fanconi and J.F. Rabolt, J. Polym. Sci. Phys. Ed. 23, 1201 (1985).

8. C.K. Chai, N.M. Dixon, D.L. Gerrard, and W. Reed, Polymer 36, 661 (1995).

9. R.P. Wool, R.S. Bretzlaff, B.Y. Li, C.H. Wang, and R.H. Boyd, J. Polym. Sci. Part B:Polym. Phys. 24, 1039 (1984).

10. D.T. Grubb and Z.-F. Li, Polymer 33, 2587 (1992).

11. D.N. Batchelder and D. Bloor, J. Polym. Sci. Polym. Phys. Ed. 17, 569 (1979).

12. X. Hu, J.L. Stanford, and R.J. Young, Polymer 35, 80 (1994).

13. J.F. Aust, K.S. Booksh, and M.L. Myrick, Appl. Spectrosc. 50, 382 (1996).

14. M.L. Myrick, S.M. Angel, R.E. Lyon, and T.M. Vess, Proc. Ann. Technical Meeting, Soc. Plastic Eng. 2052 (1992).

15. B. Fanconi, Proc. Int. Symp. Anal. and Identification of Polymers, 97 (1984).

16. N. Everall, K. Davis, H. Owen, M.J. Pelletier, and J. Slater, Appl. Spectrosc. 50,388 (1996).

17. R. Tabaksblat, R.J. Meier, and B.J. Kip, Appl. Spectrosc. 46, 60 (1992).

\section{Discussion}

Q In biological polymers electronic resonance is important even down to the VUV, which is an advantage to the Raman technique. Would you comment on extending this advantage also to the case of non-biological polymers, the C-C bond absorbs in the VUV.

A The polydiacetylenes also absorb in the VUV so that there may be some advantage to using UV excitation. However, I am not aware of any applications using UV excitation to do Raman spectroscopy of polymers. The main problem with polymers, synthetic polymers at least, is that there are always sensitive additives which tend to photolyze, giving rise to photo bleaching effects. That would argue that one should go the other way, toward longer wavelength excitation. 
Q I like to make a comment on that. I remember a study some time ago on polyacetylene of heterogeneous chain length where the excitation resonance was used to select out some population.

A Yes, exactly. That's a good point.

Q I think this case was the one where the study of the stress of the carbon-carbon triple bond was undertaken. Very large shifts, up to something like 50 to $60 \mathrm{~cm}^{-1}$ where observed. Did you look at the half widths of these bands and how the half widths change ?

A Certainly the bands change in breadth. This can be interpreted as a different distribution of stress or as a vibrational effect. 


\title{
Near-IR FT-Raman Microspectroscopy of Two Materials Systems: CVD Diamond and Zirconia Ceramics
}

\author{
Edgar S. Etz \\ Surface and Microanalysis Science Division \\ Chemical Science and Technology Laboratory \\ National Institute of Standards and Technology \\ Gaithersburg, MD 20899
}

\begin{abstract}
The application of near-infrared (NIR) excited Fourier-transform (FT) Raman microspectroscopy is illustrated toward the current study of two materials systems. These are (1) the characterization of the perfection and "quality" of synthetic, polycrystalline diamond produced by chemical vapor deposition (CVD), and (2) the investigation of yttria-stabilized zirconia powders and coatings to derive crystallographic phase information. These studies involve the use of a commercial FTRaman system, with coupled microscope, whereby the spectra are excited with the $1064 \mathrm{~nm}$ radiation of a diode-pumped Nd:YAG laser. With the examples given for both materials systems under study, it is demonstrated that the NIR excitation provides a number of analytical advantages over the study of these same systems by the more traditional approaches utilizing visible (VIS) excitation from conventional Raman sources. In the study of the CVD diamond system by FT. Raman microspectroscopy, the emphasis is on the detection and identification, with micrometer spatial resolution, of compositional heterogeneities in diamond thin films and wafers. Here the goal is to track compositional and structural defects, specifically the recognition of the various non-diamond forms of carbon, such as amorphous and graphitic carbons, predominantly at grain boundaries. New spectra are obtained that indicate greater sensitivity for non-diamond carbon impurities, through $1064 \mathrm{~nm}$ excitation, ascribed to resonance enhancement effects. The microRaman study of the zirconia system, comprising yttria-stabilized (typically 3-8 wt\% $\mathrm{Y}_{2} \mathrm{O}_{3}$ ) $\mathrm{ZrO}_{2}$ feedstock powders and plasma-spray coatings, involves the characterization of phase homogeneity and at least a qualitative determination of phase composition. In this context, three stable crystallographic phases, the monoclinic (m), tetragonal (t), and cubic (c) phase are of interest as phase composition determines the properties of the starting $\mathrm{ZrO}_{2}$ powders and the resultant thermal barrier coatings. One principal objective is also to correlate the Raman data with the results from micro-focus $\mathrm{x}$-ray diffraction (XRD) studies. Despite the long wavelength excitation, interfering laser-excited fluorescence is encountered from various commercial $\mathrm{ZrO}_{2}$ powders and coatings to make the interpretation of their spectra difficult.
\end{abstract}

\section{Introduction}

Near-infrared (NIR) Fourier transform (FT) Raman spectroscopy has contributed many advances to materials science. Among the many applications of this technique we will discuss in some detail the contributions made to the understanding of two materials systems which play an important role in contemporary technology namely, chemical vapor deposited (CVD) diamond and zirconia ceramics. Since this work involves the technique of microRaman spectroscopy coupled with FT-Raman, particular attention is given to the advantages and shortcomings in the use of NIR-excited FTRaman microspectroscopy for the micro- characterization of high technology materials.

The history of micro-Raman spectroscopy at the National Institute of Standards and Technology (NIST, formerly known as NBS) is as follows. As the name implies, micro-Raman spectroscopy involves the study of small samples with microscopes. The samples themselves are small to begin with and, additionally, it is important to assay different portions of the sample surface in order to obtain information about its structure and morphology. The methods of conventional Raman spectroscopy which, in this context, may be called macroRaman spectroscopy, can not deal with such systems, hence special techniques for studying 
microscopically sized samples had to be developed.

A proof-of-principle investigation was undertaken in the period 1973-1974 to obtain Raman spectra from microscopically sized samples with an available SPEX monochromator and photomultiplier (PMT) detection system [1]. ${ }^{1}$ Based upon this early work we developed in the period 1974 to 1976 a Raman microprobe around a J-Y Ramanor HG2 instrument with photon counting detection for single particle analysis. Further progress was made by in-house research on the design and construction of monochannel and multichannel detection microRaman instrumentation for general microanalysis using visible laser light excitation. The instruments developed were all of the nonimaging type [2,3]. This work began in 1976 and continues to the present day. Beginning in 1993 we acquired a NIR FT-Raman system with an attached fiber-optic coupled microscope. The excitation is by means of the $1.064 \mu \mathrm{m}$ radiation of the Nd:YAG laser, and we are using the instrument to define its practical utility, and its shortcomings (viz-á-viz the VIS Raman microprobe), for a broad range of materials problems. This development also continues into the present period.

The introduction of Fourier transform Raman spectroscopy, both in the visible and near infrared regions, took place at about the mid 1980s [4]. The developments in the visible region were motivated by the desire to use the advantages possessed by FT-spectrometers over those had by grating spectrographs. The extension of the technique into the near infrared, however, was strictly motivated by the need to circumvent and thus overcome the severe problems posed by the fluorescence of materials when Raman excitation was attempted with visible or even deep red laser radiation. For the most part, this move into the near infrared has been extremely successful, so much so that the appellation "FT-Raman" has become synonymous with near-infrared FT-Raman spectroscopy. We have indeed capitalized on the

1 Certain commercial equipments, instruments, and materials are identified in this paper to adequately specify the experimental procedure. Such identification does not imply recommendations or endorsements by us, nor does it imply that the materials or equipment identified are necessarily the best available for the purpose used. use of FT-Raman techniques, and I feel it useful to present some of our experiences with this method. While there are indeed many advantages there are also some drawbacks which it is good to be informed about.

The experiences we have acquired so far with the NIR FT-Raman apparatus can be summarized as follows: 1. many materials systems retain overwhelming fluorescence and luminescence interferences; 2. many types of optically absorbing materials prevent sensitive Raman response due to laser-induced sample heating and/or decomposition; 3. NIR wavelength excitation diminishes the Raman signal, by a factor of slightly more than 16 when changing from $514 \mathrm{~nm}$ to $1064 \mathrm{~nm}$, which is often detrimental to efficient micro-sampling; 4 . FT-based spectrometry offers good Raman frequency accuracy and stability, providing improved spectral matching and search techniques; 5. applications to CVD diamond materials furnish "new" spectra, ascribed to resonance enhancement effects, thereby giving greater sensitivity toward the detection of non-diamond carbon impurities, specifically amorphous and disordered graphitic carbons; and 6. applications to (yttria-stabilized) zirconia ceramics provide qualitative data on phase composition for systems impossible to study in the visible region due to intense luminescence.

The subsequent portions of this paper are organized in two parts. Part II deals with Raman spectroscopy of carbons and CVD diamond while part III is devoted to the phase identification of yttria-stabilized zirconia powders and coatings by near-infrared Fourier transform-Raman spectroscopy. The systems addressed in these two parts are of current importance to industry and technology.

\section{Raman Spectroscopy of Carbons and CVD Diamond.}

A feature of Raman spectroscopy is that most of the observed Raman spectra of solids are those of its fundamental vibrations. Vibrational overtones and combinations are also observed but the associated Raman bands are weaker and broader than those of the former. In the former case one speaks of first-order Raman scattering while the latter is designated second-order 
Raman scattering. These designations have their origin in the theory of the Raman effect and reflect the fact that the Raman bands of fundamental vibrations result from changes in the first order derivatives of the polarizability tensor with respect to the normal coordinates, while it is the second order derivatives that determine the intensities of the overtone and combination bands.

The observed characteristics of first-order Raman scattering by carbons and diamond (natural and synthetic diamond, including CVD diamond) are the following [5,6]: a) amorphous carbons are characterized by a weak, broad band extending over the region from $1100 \mathrm{~cm}^{-1}$ to $1700 \mathrm{~cm}^{-1}$, with a maximum that is located at $\sim 1550 \mathrm{~cm}^{-1}$; b) single-crystal graphite and other graphitic carbons which possess a highly ordered graphitic structure, such as pyrolytic carbons, exhibit a single Raman line at $1580 \mathrm{~cm}^{-1}$, while those of various polycrystalline disordered graphitic structures will show two more or less developed bands which can be highly diagnostic of this form of carbon. This pair of bands is commonly designated to show one peak at $\sim 1580 \mathrm{~cm}^{-1}$ (the G-band) and the other at $1355 \mathrm{~cm}^{-1}$ (the D-band). For these, the band frequencies, line widths, band shapes, and relative intensities depend on the state of phase crystallinity (microcrystalline or nanocrystalline), stress states, etc.; and c) single crystal natural diamond shows a single sharp line (with FWHM $\leq 3 \mathrm{~cm}^{-1}$ ) at $1332 \mathrm{~cm}^{-1}$, nanocrystalline diamond has peaks at $\sim 1150 \mathrm{~cm}^{-1}$ and $\sim 1480$ $\mathrm{cm}^{-1}$, while polycrystalline CVD diamond, depending on purity and structural perfection, will show a band at approximately $1332 \mathrm{~cm}^{-1}$, plus other features due to non-diamond carbon components.

Second order Raman bands are often observed for many forms of carbon, such as the first overtone band at $2700 \mathrm{~cm}^{-1}\left(2 \times 1355 \mathrm{~cm}^{-1}\right.$, the $\mathrm{G}^{\prime}$ band) and other combination modes at $\sim 2950$ $\mathrm{cm}^{-1}$ and $~ 3245 \mathrm{~cm}^{-1}$ of lower intensity.

Our work on various forms of carbon and CVD diamond was done in both the visible and infrared regions [7]. The instrumentation for the visible region used a Raman microprobe with a conventional microscope, a dispersive triple Raman spectrometer and an intensified photodiode array detection system, and argon and krypton laser excitation with wavelengths of $488.0,514.5$, and $647.1 \mathrm{~nm}$. For work in the near IR we used a commercial, Michelson interferometer-based Fourier Transform (FT) Raman system, made by Bruker Instruments, Inc., with a fiber-optic coupled Raman microscope. The excitation was accomplished with a diode pumped Nd:YAG laser oscillating at 1.064 $\mu \mathrm{m}$, while detection of the Raman signal was by means of a liquid nitrogen-cooled, near-IR germanium detector. With this equipment it was possible to cover the Stokes Raman range from 0 to $4000 \mathrm{~cm}^{-1}$ while the anti-Stokes range was limited to an upper value of $2000 \mathrm{~cm}^{-1}$, which places this limit at a wavelength of $876 \mathrm{~nm}$. The spectra were acquired at a resolution of $4 \mathrm{~cm}^{-1}$.

A broad range of carbon materials was analyzed: Carbons, graphites, and carbonaceous materials. These involved single-crystal graphite, highly-ordered pyrolytic graphite (HOPG), various disordered and amorphous carbons, commercial carbon fibers and carbon composites; a number of environmental (e.g., atmospheric, combustion) carbons, diamond-like carbon (DLC) and diamond-like nanocomposite (DLN) films, and CVD diamond samples of various origins. These were in-house specimens of various substrate-supported and free standing films. Industry-produced research samples such as thin film specimens and wafers obtained from Crystallume, Norton Diamond Film, and the Raytheon Research Division were also studied, as were thermal conductivity round robin specimens which included various sets of CVD diamond wafers with measured thermal conductivities in the range from 4.0 to 17.0 $\mathrm{W} / \mathrm{cm}-\mathrm{K}$.

The characterization of CVD diamond by means of micro-Raman spectroscopy had these aims. It was desired to correlate the Raman spectra with: Deposition conditions such as the composition of the $\mathrm{H}_{2}: \mathrm{CH}_{4}$ gas mixture and the system pressure and substrate temperature; nucleation and growth rates; particle and film morphologies; non-diamond carbon impurities such as disordered graphitic carbons and amorphous carbon; structural defects due to lattice vacancies and interstitials, strain and stress effects, and impurity atoms $(\mathrm{Si}, \mathrm{N}, \mathrm{H})$; and film heterogeneity, both laterally, across the area, and longitudinally, across the thickness. 
Figure 1 shows typical spectra of natural diamond ( $\mathrm{sp}^{3}$ bonding), graphite ( $\mathrm{sp}^{2}$ bonding), and disordered graphitic carbon (amorphous carbon, sp bonding). These spectra illustrate the specificity for characterizing carbon materials by Raman spectroscopy. The Raman spectrum of natural diamond has a low background and a sharp line at $1332 \pm 1 \mathrm{~cm}^{-1}$. This line is sensitive to lattice strain. The diamond-like carbons (DLC) do not show this band. In graphite the line occurs at $1582 \mathrm{~cm}^{-1}$ and it is broader than for natural diamond. Amorphous carbon consisting of various disordered structures has two broad bands centered at $\sim 1580 \mathrm{~cm}^{-1}$ (G-band) and at $\sim 1355 \mathrm{~cm}^{-1}$ (D-band). In the laser excitation of the spectra of such carbons, especially with the micro-focus Raman technique, excessive laser irradiance focused at the sample may easily induce structural changes (such as graphitization) through sample heating, to drastically change the appearance of these bands. Thus the laser power, reflectivity of the sample, and its absorptive properties are critical to the interpretation of these spectra. Figure 2 illustrates the results possible with micro-Raman

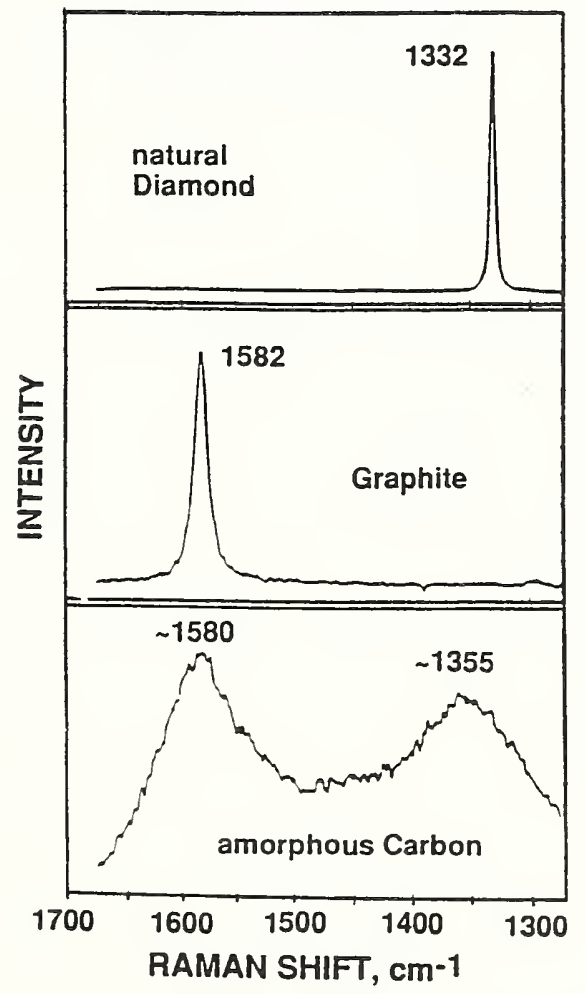

Fig. 1. Typical Raman spectra of carbon materials. spectroscopy. It shows the Raman spectrum of a single carbon fiber of diameter $7 \mu \mathrm{m}$ which was generated by the visible argon laser line at 514.5 $\mathrm{nm}$. The focused laser spot had a diameter of $\sim 2$ $\mu \mathrm{m}$ and the laser power at the sample was $6 \mathrm{~mW}$. The spectrum is clean, on a low background and shows the characteristic $D, D^{\prime}, G$ and $G^{\prime}$ bands plus several other weak bands.

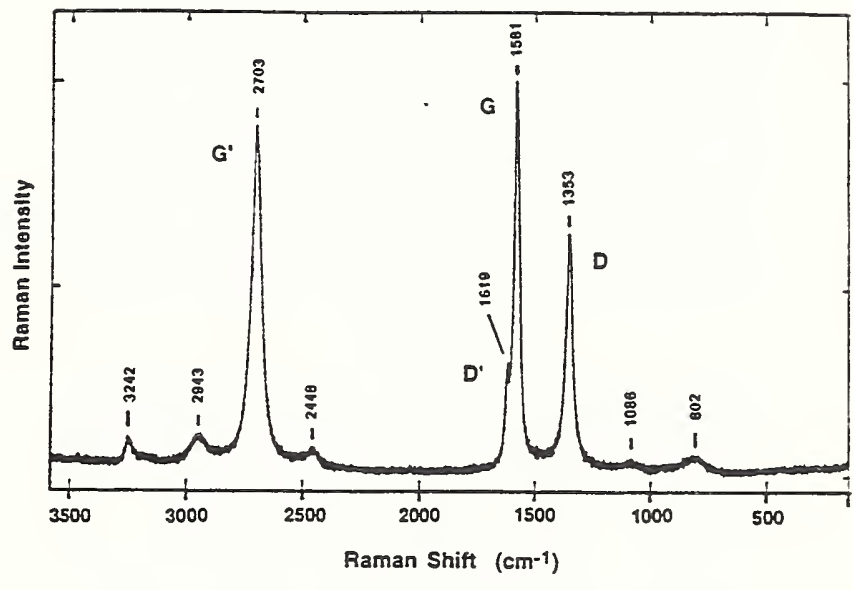

Fig. 2. Micro-Raman spectrum of a single carbon fiber of diameter $7 \mu \mathrm{m}$.

One of the difficulties experienced with opaque samples is that of sample heating due to absorption of laser radiation. To be sure, there is of course also Raman scattered radiation and Fig. 3 illustrates the effect of laser heating on the NIR Raman spectrum. Conventional illumination of the sample (laser spot diameter 200 $\mu \mathrm{m})$ was used together with the NIR FT-Raman technique $\left(\lambda_{0}=1064 \mathrm{~nm}\right)$. The spectra are quite weak and the effect of laser heating is quite pronounced.

There are several methods of producing thin films, and free-standing wafers, of CVD diamond. The three most widely used techniques involve deposition by (i) hot filament assisted CVD, (ii) microwave assisted CVD, and (iii) arc jet plasma growth. These lead to the formation of thin films on suitable substrate materials (e.g., single-crystal silicon, alumina, carbides, metals), from which can be 

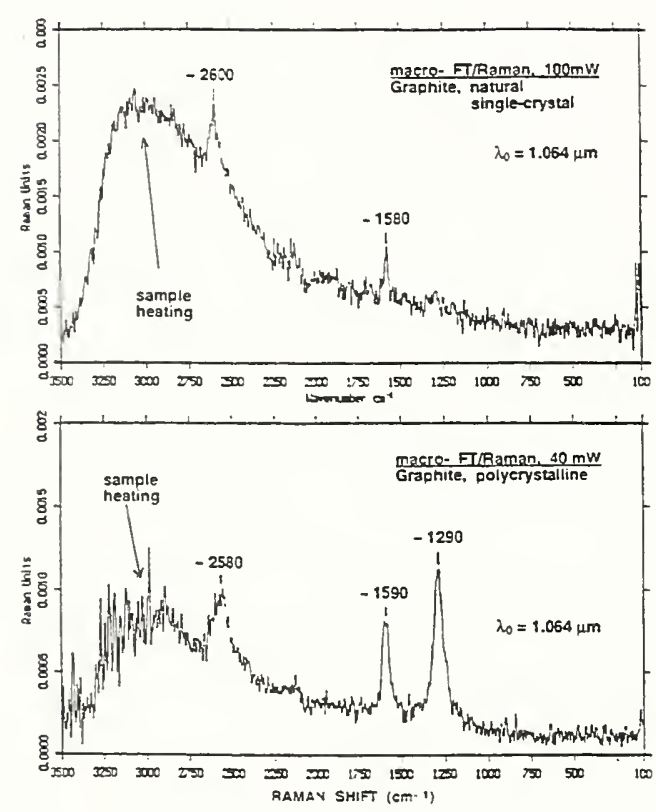

Fig. 3. FT-Raman spectra of two graphite samples excited at $1.064 \mu \mathrm{m}$ with the diameter of the laser spot on the sample expanded to a diameter of $200 \mu \mathrm{m}$.

produced free-standing wafers that are now available commercially as optical windows in thicknesses up to $1 \mathrm{~mm}$.

The formation of polycrystalline diamond by CVD processes is dependent on the deposition conditions. Raman spectroscopy is sensitive to non-diamond carbon impurities, such as graphitic and amorphous carbons. The level of these impurities is in part determined by the feed gas composition, which is typically 0.2 to $2.0 \%$ $\mathrm{CH}_{4}$ in $\mathrm{H}_{2}$, and pressure, as well as to substrate temperature, which ranges anywhere from $700^{\circ} \mathrm{C}$ to $1200^{\circ} \mathrm{C}$. These dependencies are illustrated by the Raman spectra in Fig. 4. We see drastic changes in the Raman spectra excited at 514.5 $\mathrm{nm}$. For these, the band seen at $520 \mathrm{~cm}^{-1}$ is the Raman line of the silicon (single crystal material) substrate. Changing the methane concentration from $0.5 \%$ to just $1 \%$ in hydrogen at a total pressure of $10.66 \times 10^{3} \mathrm{~Pa}$ (80 torr) and at essentially the same substrate temperature $\left(\sim 990^{\circ} \mathrm{C}\right)$ causes the $1332 \mathrm{~cm}^{-1}$ line to broaden and to now be located on top of a rising spectral background with a developing broad band centered at $\sim 1550 \mathrm{~cm}^{-1}$. Further changes in the deposition conditions cause additional changes in the Raman spectrum as is shown in the two right hand panels of the figure, e.g., a nondiamond carbon feature shows up at $1597 \mathrm{~cm}^{-1}$ (lower right hand panel). The 1574 and 1346 $\mathrm{cm}^{-1}$ bands in the top right hand panel show that at the stated deposition condition, the deposited film no longer fits the description of a "clean" diamond film, but rather exhibits the spectral characteristics of predominant non-diamond carbon impurities which are not, however,

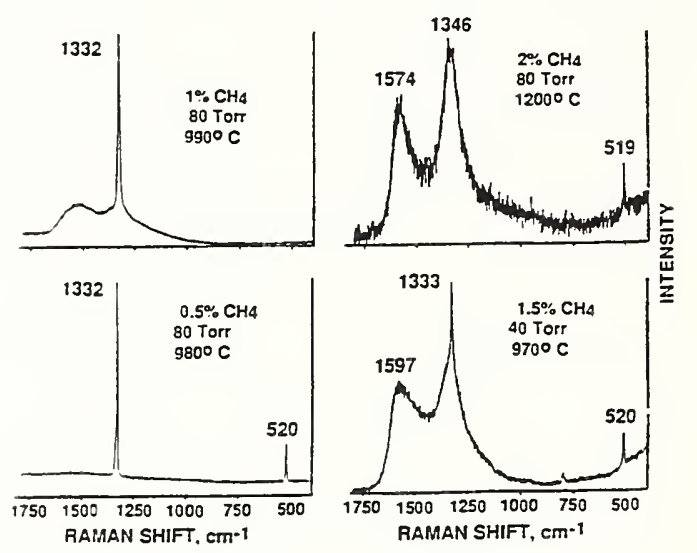

Fig. 4. Raman spectra of diamond films on $\mathrm{Si}$ substrates showing the effect of deposition conditions. (Adapted from Ref. 6.)

readily quantifiable. Thus, the relative intensities, frequencies, and line widths of the Raman bands can serve only as qualitative indicators of defect carbon structures.

The typical surface morphology of diamond films on silicon substrates is depicted by several scanning electron micrographs shown in Fig. 5. The top micrograph shows the film in its early stages of nucleation, with largely unconnected, fully faceted, three-dimensional, single microcrystallites. As nucleation progresses, these crystallites coalesce and form a continuous thin film. A continuous film, of average thickness $\sim 9 \mu \mathrm{m}$, is shown in the middle micrograph, with crystallites of various imperfect geometric structures (from secondary nucleation), and numerous grain boundaries potential sites of non-diamond constituents. The bottom micrograph shows that same film in cross section, indicating a columnar growth pattern, with average grain size increasing from the initial growth surface (the interface with the $\mathrm{Si}$ substrate) to the final, top surface of the film. Such films are typically "cleaner" (consisting of 

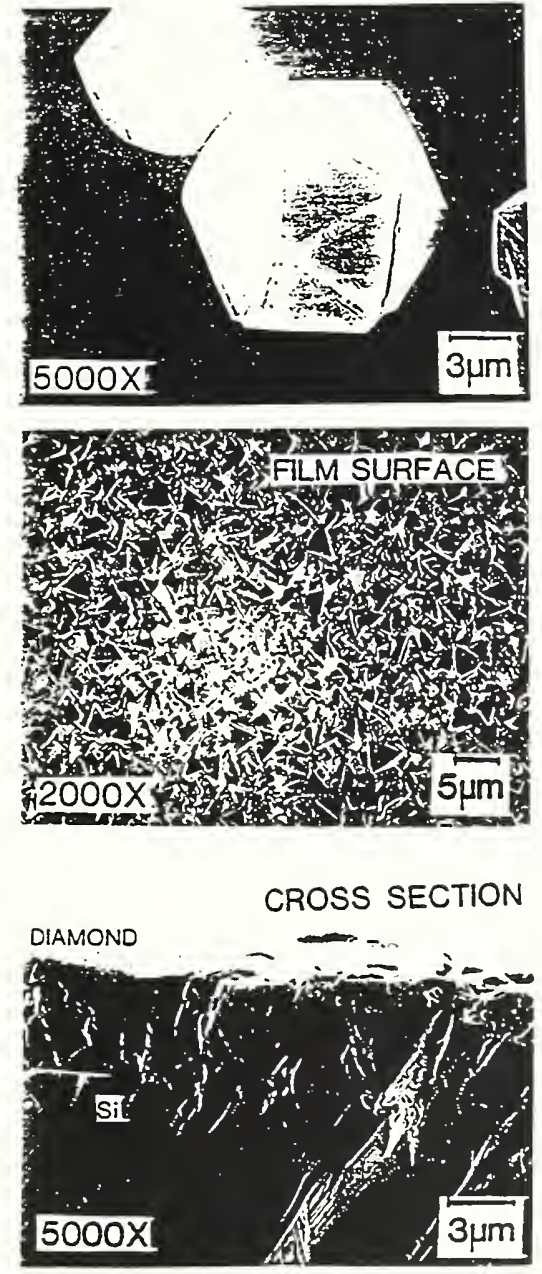

Fig. 5. Scanning electron micrographs of CVD diamond films on a silicon substrate.

a "better" diamond) in the top surface than in their early growth strata adjacent to the substrate. Micro-Raman measurements across such cross sections are therefore very informative.

The Raman spectra of CVD diamond particles (i.e., microcrystallites) and thin films are compared in Fig. 6. Here the sample was one grown at NIST. The spectra were excited by visible argon or krypton laser lines. The top panel shows the Raman spectrum of a single micro particle from CVD grown diamond deposit on a silicon wafer substrate. The particle size was $\sim 12 \mu \mathrm{m}$. The $1333 \mathrm{~cm}^{-1}$ line is strong and fairly sharp with a width of ca. $6 \mathrm{~cm}^{-1}$ on a weak and fairly flat background. There is no indication of the presence of any non-diamond carbon. Also seen are the $521 \mathrm{~cm}^{-1}$ and $965 \mathrm{~cm}^{-1}$ phonon lines of the silicon substrate. In the indicated second spectral region, much beyond the normal Raman range, we observe upon the spectral background a weak feature at $5890 \mathrm{~cm}^{-1}$ (Raman shift) or at $1.68 \mathrm{eV}$. This laser-excited photoluminescence is a well known feature in spectra of this type and is commonly referred to as the $738 \mathrm{~nm}$ defect luminescence band, believed to be due to a silicon impurity in the diamond lattice.

The lower panel shows the Raman spectrum of a diamond film of $5 \mu \mathrm{m}$ thickness, and there are expected changes from the spectrum of a perfect single diamond microcrystal. The film spectrum now exhibits the general features characteristic of microstructural and compositional imperfections of the diamond phase. The diamond line at $1334 \mathrm{~cm}^{-1}$ now rides on top of a sloping
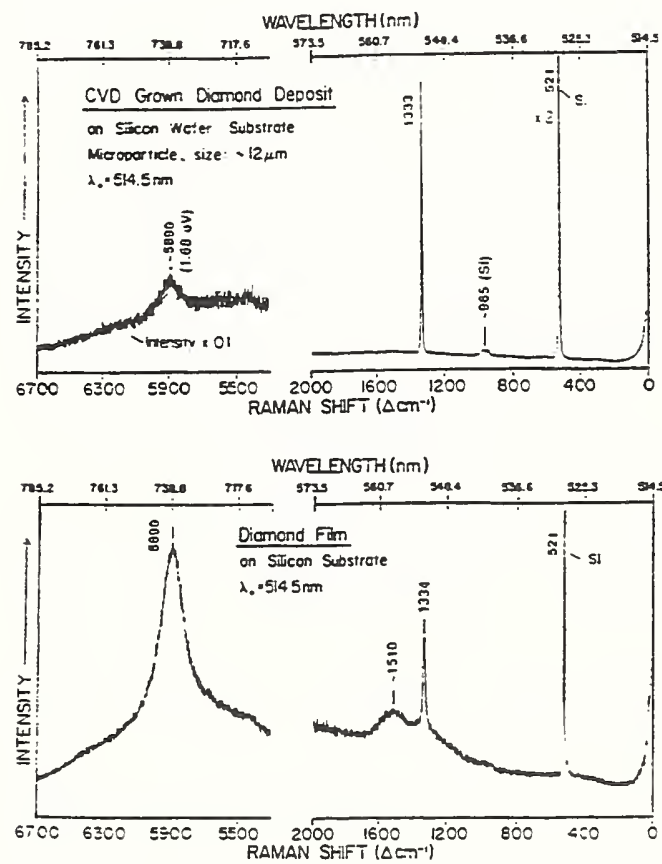

Fig. 6. Micro-Raman spectra of NIST CVD diamond on a silicon wafer employing visible excitation.

background and there is still the silicon phonon line at $521 \mathrm{~cm}^{-1}$ which indicates that the film is indeed thin and transparent to the laser line. The sloping background reaches a peak at $1510 \mathrm{~cm}^{-1}$ which indicates the presence of non-diamond carbon components in the film. The photoluminescence band at $5890 \mathrm{~cm}^{-1}$ is seen to be strongly enhanced. 
We also studied a sample of a free-standing wafer polished to high optical finish on both of its surfaces, provided by Norton Diamond Film of Massachusetts. An optical micrograph of this wafer (thickness: $530 \mu \mathrm{m}$ ) is shown in Fig. 7. The top panel shows the morphology of the top and bottom surface, respectively, while the bottom panel shows that of the cross section. The top surface consists of large grains, the typical dimensions of which range from $60 \mu \mathrm{m}$ to 300 $\mu \mathrm{m}$. The bottom surface, however, consists of small grains. The size of the grains in the top surface of such films depends on to what thickness the film has grown, so that the grains can be very large. It follows also that the top surface contains proportionately less, or fewer, grain boundaries, than are present in the bottom surface. The lower micrograph presents that same wafer in cross section (fractured, not polished) which gives a side view of the grain

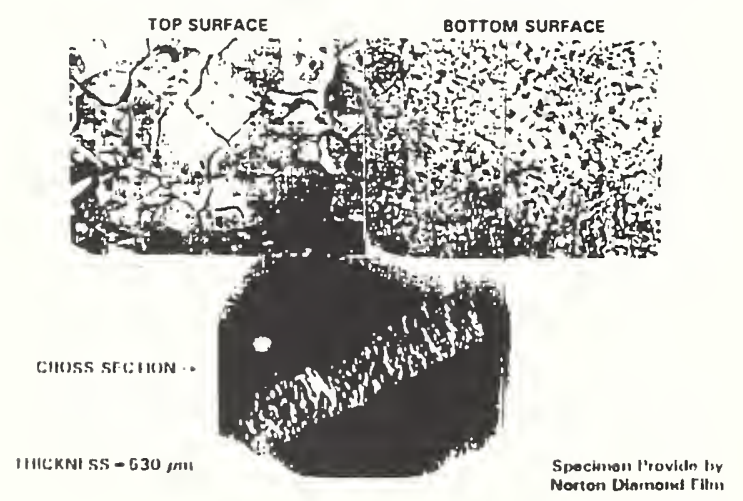

Fig. 7. Optical micrographs of a diamond wafer of thickness $530 \mu \mathrm{m}$. Both top and bottom surfaces are polished to optical quality.

morphology. Figure 8 shows the representative spectra from Raman microprobe measurements (with excitation at $514.5 \mathrm{~nm}$ ) on the fracture surface of the wafer cross section, where the laser spot probes the top and bottom surface layer, respectively. There are two distinctly different qualities of diamond in the top surface layer and the bottom surface layer of the wafer, respectively, with gradations in between. The large-grain top surface is relatively free of nondiamond carbon while the small-grain bottom surface is defect rich in amorphous carbon which

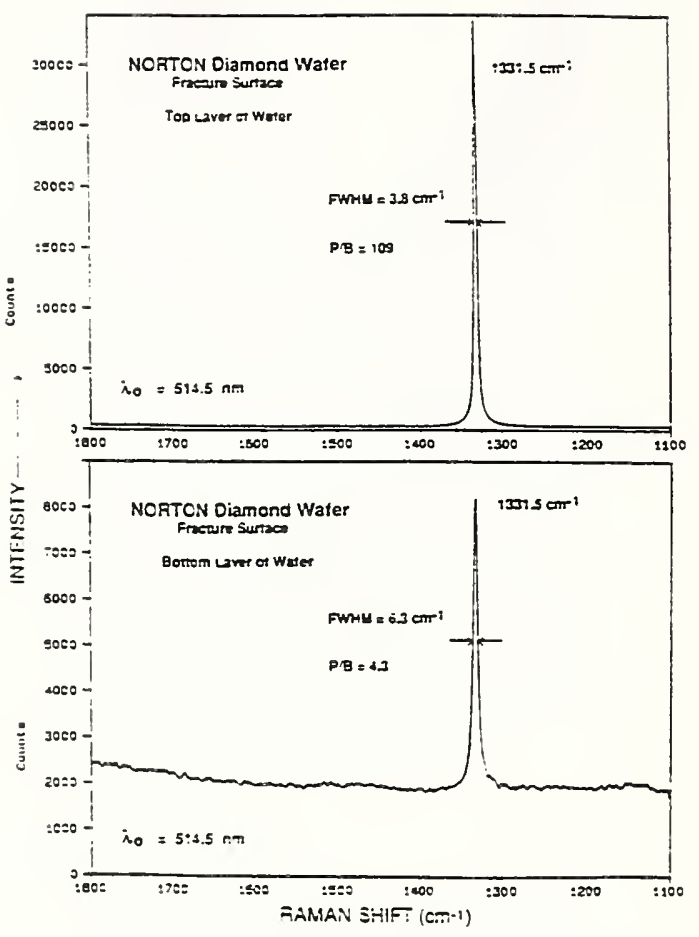

Fig. 8. Micro-Raman spectra of industry-produced CVD diamond. The sample was provided by Norton Diamond Film, sample No. TD846M. The spectra were obtained with visible laser excitation.

is predominantly located at the grain boundaries. The top layer is seen to be of excellent quality, the Raman line having a width of $3.8 \mathrm{~cm}^{-1}$ and the whole spectrum having a very low background. In contrast to this we see that the bottom spectrum rides on top of a fairly large and sloping background and that the line width is $6.3 \mathrm{~cm}^{-1}$. As a semi-quantitative measure of quality of the diamond we use the peak-tobackground ratio (P/B) which, in this case, are the intensities taken at $\sim 1332 \mathrm{~cm}^{-1}$ and $\sim 1500$ $\mathrm{cm}^{-1}$. For the top and bottom layers these are $\mathrm{P} / \mathrm{B}=109$ and $\mathrm{P} / \mathrm{B}=4.3$ respectively.

It should be noted that the Raman spectrum of this commercial sample of high optical quality CVD diamond, generated with VIS excitation at either $488.0 \mathrm{~nm}$ or $514.5 \mathrm{~nm}$, when obtained from the top surface of the wafer, is virtually indistinguishable from the corresponding spectrum of the highest quality Type IIa natural diamond (a luminescence-free specimen we use as an in-house diamond reference "standard"). 
Thus, for diamond of this quality, visible excitation is at the limit of detecting such low compositional impurities and structural imperfections.

We now consider the FT-Raman spectra of diamond and CVD diamond. One would expect
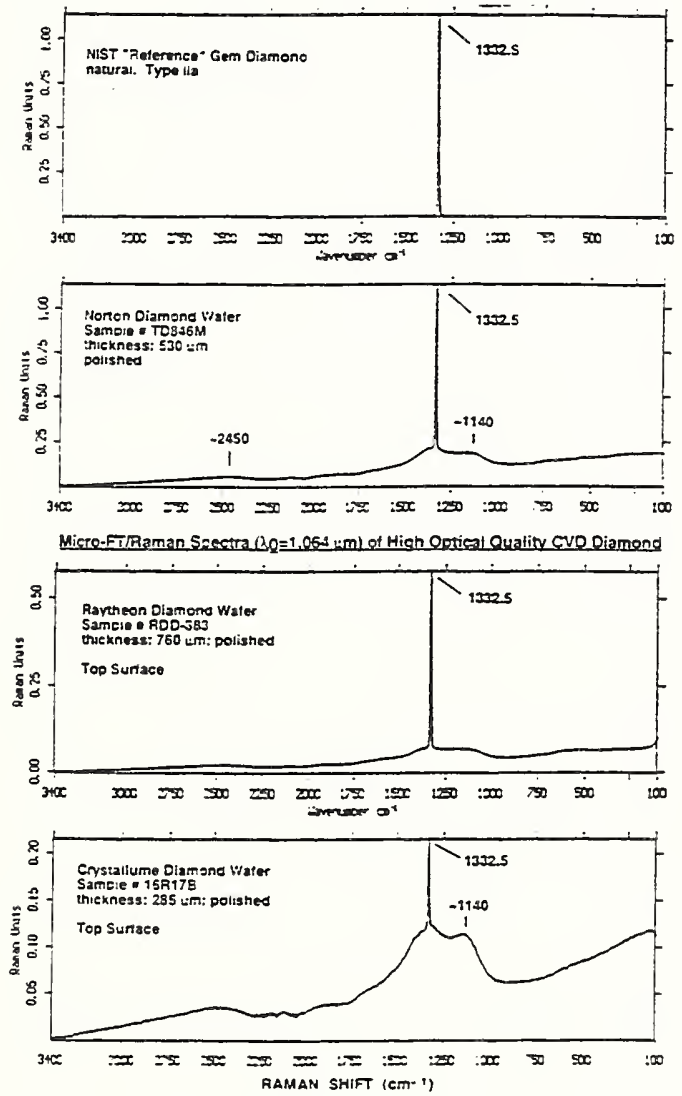

Fig. 9. FT-Raman spectra $\left(\lambda_{0}=1.064 \mu \mathrm{m}\right)$ of a gem quality diamond and several CVD diamond wafers.

that there be no difference in the results that are obtained from visible light excitation. However, Fig. 9 shows some FT-Raman spectra which allow one to draw distinctions between the spectra generated with visible light. Here we have diamond samples of high optical quality which were examined in the FT-Raman microscope with near-IR excitation $\left(\lambda_{0}=1064\right.$ $\mathrm{nm})$. The top spectrum is that of a natural gem diamond, a NIST "Reference" material. The spectrum is absolutely perfect; the background is near zero and the Raman line is extremely sharp. The next lower panel shows the spectrum of the same Norton wafer whose visible light excited spectra are shown in Fig. 8. We see that even though the spectra excited with visible radiation are indistinguishable from that of FT-Raman spectrum of the gem-quality diamond (top of Fig. 9), there are significant differences with respect to the spectrum shown in the second panel of Fig. 9, where the $1332 \mathrm{~cm}^{-1}$ line now rides on top of a broad background which, in the first-order carbon region, shows bands with maxima at $\sim 1140 \mathrm{~cm}^{-1}$ and $\sim 1350 \mathrm{~cm}^{-1}$. These general features are also present in the spectra of the Raytheon and the Crystallume wafers and, depending on diamond "quality" or "perfection", are more or less pronounced. These two bands can be attributed to amorphous or disordered carbon components. However, the origin of the general luminescence background upon which these bands are observed is not understood. The three industrial CVD diamond specimens of Fig. 9 , being wafers of high optical quality are virtually colorless and water-clear ("white diamond"), as is the single-crystal gem diamond. Yet, they show this luminescence upon NIR excitation. As such optical grade CVD material deteriorates in appearance from a slight browngrey hue to a progressively more grey, or even dark-grey appearance indicative of abundant defects and impurities (especially both increased hydrogen and nitrogen trace components) then these same spectral features are so pronounced as to entirely obscure the $-1332 \mathrm{~cm}^{-1}$ diamond line. We have correlated these spectra with the thermal conductivity properties of such wafers and found that high values of thermal conductivity (i.e., $\mathrm{K}$ values in the range from 12 to $17 \mathrm{~W} / \mathrm{cm}-\mathrm{K}$ for the optical grade material) correlate with the FT-Raman spectra of the type shown in Fig. 9. The spectra of these three commercial wafers are practically indistinguishable from one another when produced by either the argon $488 \mathrm{~nm}$ or $514 \mathrm{~nm}$ radiations. These differences then demonstrate that NIR FTRaman spectroscopy is capable of distinguishing, with greater sensitivity, among CVD diamond material (films and wafers) of differing quality.

Recording the micro FT-Raman spectrum of the top and bottom surfaces of the Norton wafer, we have the results shown in Fig. 10. Here this specimen was again examined in cross-section (fracture surface), with spectrum (a) obtained 
when the probe spot was near the edge of the top surface (growth side) and (b) being that from the near edge of the bottom surface (the former substrate side). These two surface

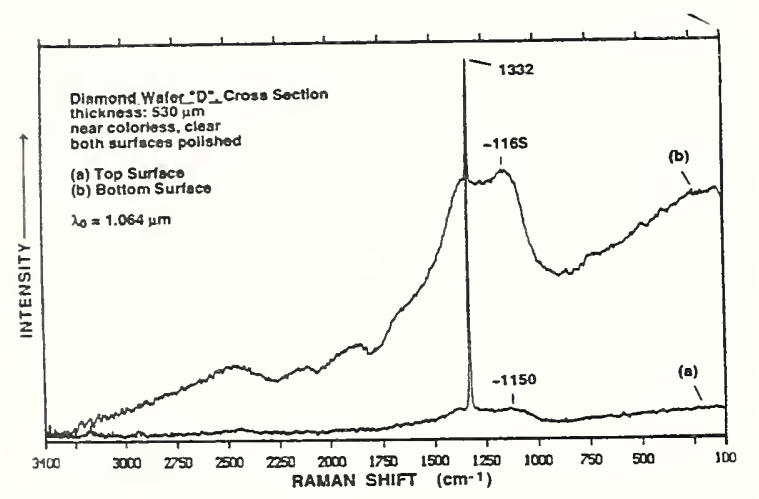

Fig. 10. Micro-FT-Raman spectrum of the Norton wafer (sample No. TD846M) taken in cross section at the top (a) and the bottom (b) surface.

layers are about $500 \mu \mathrm{m}$ away from one another (for the wafer thickness of $530 \mu \mathrm{m}$ ). From the superimposed spectra, we note the drastic differences in the relative quality or perfection of the diamond material, for the top surface (a) $v s$. The bottom surface (b). The bottom surface spectrum is dominated by a pronounced luminescence background, whereby the broad band with merged peaks at $\sim 1165 \mathrm{~cm}^{-1}$ and $\sim 1350 \mathrm{~cm}^{-1}$ nearly obscures the diamond line, whereas the spectrum of the top surface, from large-grain material, indicates a high-quality clean diamond. In fact, microprobe spectra taken at grain boundaries in the top-surface layer show the spectral features indicative of poor diamond material. Thus, without question, much of the non-diamond carbon impurities and defects are residing and are concentrated at the grain boundaries. It is through the NIR FT-Raman approach that greater analytical sensitivity is obtained for the detection of these imperfections augmented by the spatially resolved measurements performed with the Raman microscope. The increased sensitivity for nondiamond, $\mathrm{sp}^{2}$-bonded carbons, on NIR excitation, is attributed to resonance enhancement [8].

\section{Phase Identification of Yttria-Stabilized Zirconia Powders and Coatings by Near-IR FT-Raman Spectroscopy.}

The work reported in this part of the paper represents the Raman contribution to a larger study of the zirconia ceramic system that is aimed at producing NIST Standard Reference Materials (SRMs) for several chemical and physical properties defined by the needs of this industry. The Raman results discussed here are preliminary and presently await full correlation with the results from other characterization techniques, specifically (i) $\mathrm{x}$-ray microanalysis and electron microscopy, (ii) $\mathrm{x}$-ray diffraction, both macro- and micro-, and (iii) neutron diffraction analysis. The Raman measurements have been performed on a large variety of zirconia powders and coatings, largely obtained from several industrial producers, and represent the types of materials and products that industry is dealing with. As such, these test samples have been obtained from powder producers including Tosoh, Metco, Metec, Zircoa, and finished coatings from Pratt\&Whitney, SUNYStoney Brook, and Sandia National Laboratories.

Stabilized zirconias are important technological materials for which the control of microstructure and phase composition is of great importance as these largely govern the performance of the resulting coatings. Zirconia coatings produced by thermal spray coating methods are important as functionally gradient materials. Towards this application, Raman spectroscopy is not a quantitative technique which, nevertheless, is able to provide useful phase information.

Common zirconia feed powders and finished coatings are usually characterized by bulk $\mathrm{x}$-ray diffraction and sometimes by neutron diffraction methods for phase composition. Often enough, however, the best results on phase composition thus obtained are inconsistent and inconclusive. In view of this fact, there is a need for reference materials that can serve as standards for comparison. It therefore was suggested that micro-Raman spectroscopy be used for qualitative assessment of phase composition for feedstock source zirconia powders and finished coatings. These studies were to be done for a series of yttria-stabilized $\mathrm{ZrO}_{2}$ powders with 
typical $\mathrm{Y}_{2} \mathrm{O}_{3}$ contents from $3-8 \mathrm{wt} \%$, which have been characterized by various physical (e.g., particle size distribution, density, etc.) and chemical methods (e.g., elemental analysis, bulk $\mathrm{x}$-ray and neutron diffraction) and have the Raman spectra correlate with these properties.

The objectives of this research were, then, the following. 1) To obtain Raman spectra for phase identification and correlate them with the results obtained from bulk- and micro- x-ray diffraction and neutron diffraction analyses; 2) record Raman spectra for source zirconia powders and relate these to the spectra from corresponding ceramic coatings; 3 ) examine source powders by macro- and micro-Raman spectroscopy for heterogeneity of composition; 4) study sizesegregated Metec zirconia powders (prototype NIST Standard Reference Materials) for homogeneity of phase composition by microRaman spectroscopy; and 5) correlate the microRaman data on the Metec powders with microfocus $\mathrm{x}$-ray diffraction patterns for single particles.

Raman spectra of zirconia have been reported over the last two decades [9-11]. Three structures for a number of polymorphs of $\mathrm{ZrO}_{2}$ have been well established to exist at atmospheric pressure: a monoclinic (m) structure with distinguishing bands at $182 \mathrm{~cm}^{-1}$ and $193 \mathrm{~cm}^{-1}$, a tetragonal (t) structure with characteristic bands at $\sim 148 \mathrm{~cm}^{-1}$ and $\sim 260 \mathrm{~cm}^{-1}$, and a cubic (c) structure with just one band at $\sim 607 \mathrm{~cm}^{-1}$. However, it was not always possible to determine the absolute phase purity or the relative proportions of phases in mixed phase systems. Quantitative Raman measurements on this system have not been reported.

In trying to record the Raman spectra of zirconia one is often confronted with the problem of sample luminescence/fluorescence. This is a complicating factor for many of these materials, both powders and coatings, and results from luminescing impurities (usually trace concentrations of rare earth ions) incorporated in the zirconia matrix. Also, strain and stress induced luminescence may be a factor. Visible laser excitation (typically at $\lambda_{0}=488.0,514.5$, and $647.1 \mathrm{~nm}$ ) often brings on overwhelming sample fluorescence. Near-IR excitation at $1.064 \mu \mathrm{m}$ (Nd:YAG laser), in principle, reduces, or fully avoids, laser-induced sample fluorescence. Raman spectra were therefore generated under these experimental conditions: a commercial interferometric FT-Raman system equipped with a sample stage for macro-, or bulk, measurements with a laser spot size on the sample from $\sim 100 \mu \mathrm{m}$ to $\sim 200 \mu \mathrm{m}$ and an optic fiber-coupled Raman microscope was employed for microsampling measurements of probe spots with a diameter of $\sim 5 \mu \mathrm{m}$ to $\sim 65 \mu \mathrm{m}$. All spectra were excited with the $1.064 \mu \mathrm{m}$ line of a $\mathrm{Nd}$ :YAG laser with power levels from $50 \mathrm{~mW}$ to $600 \mathrm{~mW}$ for the macro-measurements and from $20 \mathrm{~mW}$ to $300 \mathrm{~mW}$ for the micro-experiments. The spectra were acquired over the Stokes/antiStokes Raman range from $3500 \mathrm{~cm}^{-1}$ to $-2000 \mathrm{~cm}^{-1}$ at a resolution of $4 \mathrm{~cm}^{-1}$.

Various types of yttria-stabilized zirconia powders, prepared by proprietary methods using feedstock materials for thermal-barrier coatings, were used. "Reference samples" of $\mathrm{ZrO}_{2}$, both monophase and mixed-phase materials of "known" composition were used for comparisons. Both planar sections and cross sections of zirconia coatings (generally on steel plate substrates) produced by thermal spray coating methods were employed. The most extensive measurements were made on a set of Metec zirconia powders stabilized by $\sim 8 \mathrm{wt} \%$ $\mathrm{Y}_{2} \mathrm{O}_{3}$, that had been supplied as a powder of a broad, polydisperse size distribution (particle size $\sim 2 \mu \mathrm{m}$ to $\sim 80 \mu \mathrm{m}$ ) and as powders of three size fractions. For these, it was of interest to study phase composition as a function of the size regime of the powder, and to prepare the plasmaspray coatings from the size-segregated feedstock powders. The non-size-fractionated Metec zirconia powder is extensively being evaluated for its suitability as a Standard Reference Material [12].

An example of the kind of Raman spectra that were studied with conventional techniques is shown in Fig. 11. The top frame is a spectrum for the tetragonal phase while the spectrum for the monoclinic phase is shown below. We note the differences between them, which allows qualitative statements about the phase composition, provided such clean spectra are obtained with low spectral backgrounds. These same phase-pure research samples of high purity furnish identical spectra when excited at $1.064 \mu \mathrm{m}$. Figure 12 presents a 
scanning electron micrograph of yttrium stabilized zirconia feedstock powder showing the kinds of particles and their size distributions studied. Even at the single particle level, various degrees of compositional heterogeneity, in regard to phase composition, are encountered.

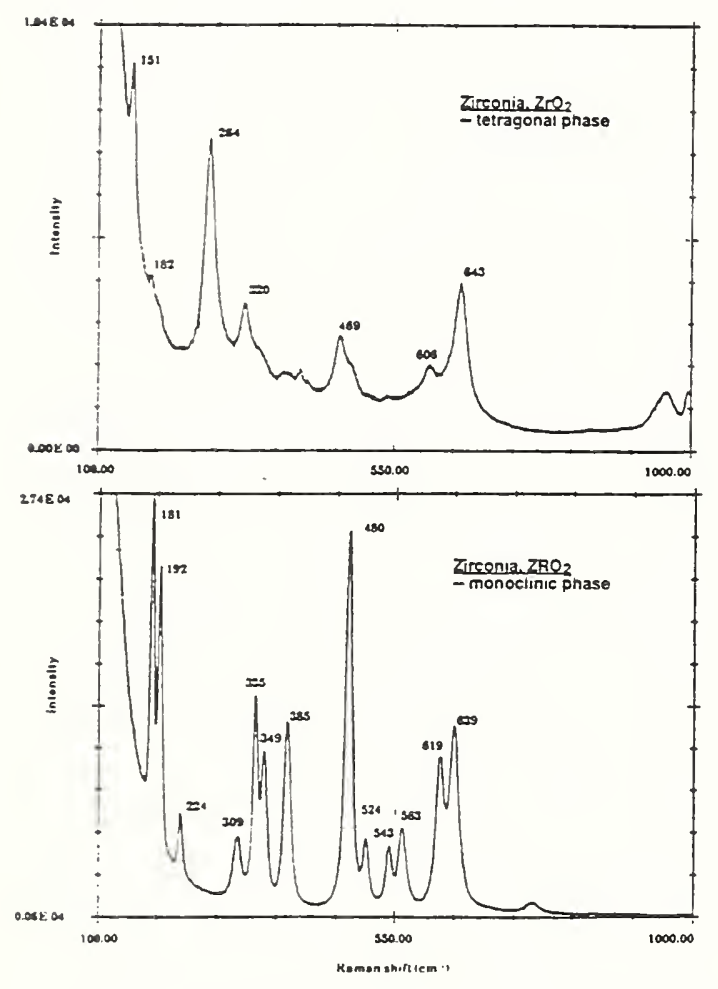

Fig. 11. Raman spectra of tetragonal (top) and monoclinic (bottom) $\mathrm{ZrO}_{2}$ obtained with visible excitation [from Bowden, et al., ref. 13]

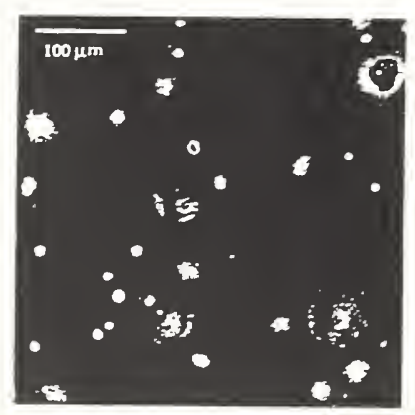

Fig. 12. Scanning electron micrograph of $\mathrm{ZrO}_{2}$ feedstock powder used for the fabrication of thermalbarrier coatings. [Sample no. Metco- $\mathrm{P}, 8 \mathrm{wt} \% \mathrm{Y}_{2} \mathrm{O}_{3}$ ].

An attempt to record a Raman spectrum for one of these commercial feedstock $\mathrm{ZrO}_{2}$ powders, namely the non-size-segregated Metec powder, using the argon laser line at $514.5 \mathrm{~nm}$, is shown in Fig. 13. Here the spectrum, basically the luminescence emission spectrum, was acquired from $500 \mathrm{~nm}$ to $1000 \mathrm{~nm}$, where for the Raman range (from the exciting line out to $\sim 628 \mathrm{~nm}$, i.e., $3500 \mathrm{~cm}^{-1}$ Raman shift) no Raman bands could be observed due to intense sample luminescence. There is even some luminescence structure that goes out to $1000 \mathrm{~nm}$.

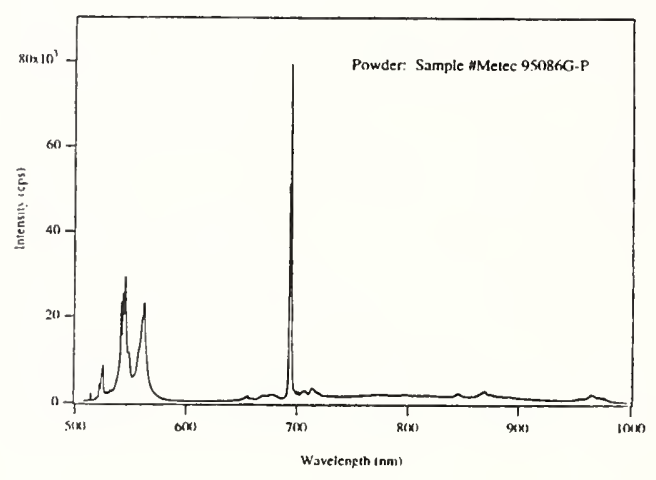

Fig. 13. Luminescence spectrum of $\mathrm{ZrO}_{2}$ powder excited by the argon $514.5 \mathrm{~nm}$ line.

The typical spectra that are obtained by us with our Bruker FT-Raman system are shown in Fig. 14. The top two spectra span the (Stokes) range from 0 to $800 \mathrm{~cm}^{-1}$, while the spectrum for the zirconia coating is shown from 0 to $3300 \mathrm{~cm}^{-1}$ (the fall-off of the Ge detector). In this latter spectrum, the intense photoluminescence makes only the major Raman bands observable, which for this coating, are the Raman bands of the desired tetragonal ( $t$ ) phase. Thus, even with near-IR excitation, i.e., $1.064 \mu \mathrm{m}$, the luminescence problem is not overcome. One other complication in any attempt to vaguely quantify these spectra as to phase composition is the fact that Raman scattering has greater sensitivity for the monoclinic phase due to a relatively stronger scattering cross section for this phase [11]. Further difficulties toward quantitation, especially in microsampling, arise from morphology and surface roughness effects.

Figure 15 shows the characteristic FT-Raman spectrum of the candidate Standard Reference Material, the Metec \#95086G powder (not size- 
fractionated), an yttria-stabilized $\mathrm{ZrO}_{2}$ powder of mixed composition. Neutron diffraction

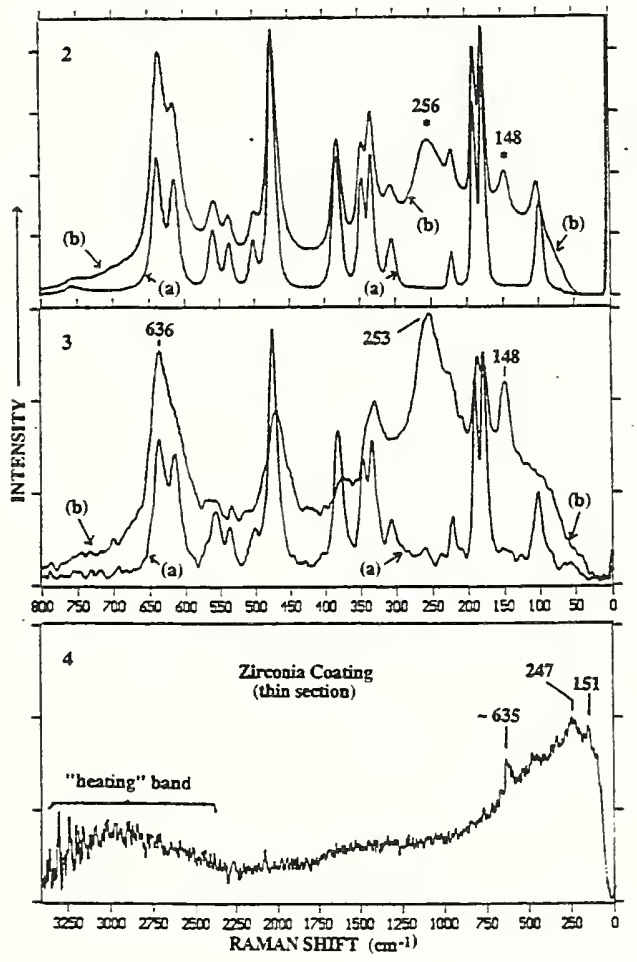

Fig. 14. Near-IR FT-Raman spectra of yttriastabilized zirconia powders and coatings $\left(\lambda_{0}=1.064 \mu \mathrm{m}\right)$.

Top: macro-spectra of (a) pure phase monoclinic $\mathrm{ZrO}_{2}$ powder (unstabilized), and (b) mixed-phase monoclinic/tetragonal stabilized $\mathrm{ZrO}_{2}$ powder $(8 \mathrm{wt} \%$ $\mathrm{Y}_{2} \mathrm{O}_{3}$ ). Bands marked by asterisks $\left({ }^{*}\right)$ are characteristic of the tetragonal component.

Middle: Micro-spectra showing phase inhomogeneity of feedstock zirconia powder $\left(8 \mathrm{wt} \% \mathrm{Y}_{2} \mathrm{O}_{3}\right)$ for two microspheres: (a) Particle 1 (size: $43 \mu \mathrm{m}$ ), largely monoclinic $\mathrm{ZrO}_{2}$, and (b) Particle $2(28 \mu \mathrm{m})$ mainly tetragonal material. No cubic phase component was detected in any of the particles analyzed.

Bottom: Micro-spectrum of thermal-barrier zirconia coating (8 wt\% $\left.\mathrm{Y}_{2} \mathrm{O}_{3}\right)$ analyzed as a planar thin section (thickness: $30 \mu \mathrm{m}$ ). Luminescent background partially obscures Raman features: "heating band" indicates laser-induced sample heating. The coating emits intense photoluminescence in Raman experiments with $514.5 \mathrm{~nm}$ excitation, thereby making the Raman spectrum unobservable. analysis shows all three phases to be present. Both the Stokes and anti-Stokes sides of the $1.064 \mu \mathrm{m}$ excited spectrum are shown. Surprisingly, all parts of the anti-Stokes spectrum show intense photoluminescence, in addition to the $2861 \mathrm{~cm}^{-1}$ band on the Stokes side. The Stokes side between the Rayleigh line and $700 \mathrm{~cm}^{-1}$ still exhibits the roll-off of the luminescence on the anti-Stokes side, but also reveals useable Raman shifts. The region around

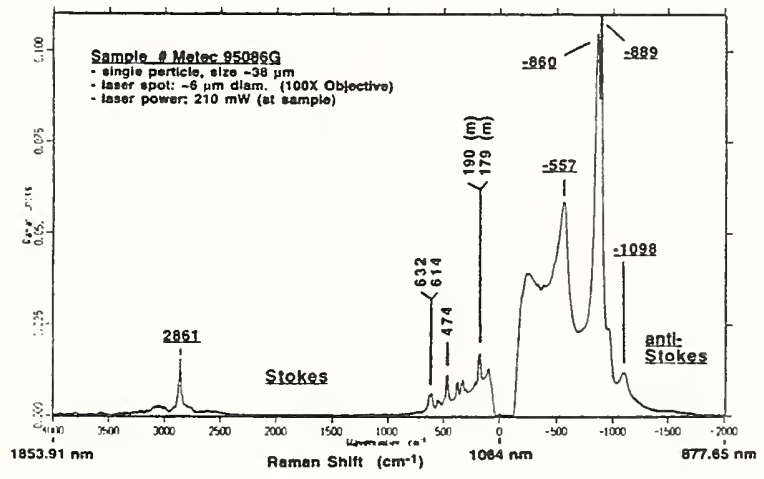

Fig. 15. Micro-FT-Raman spectrum of yttriastabilized zirconia powder, $\mathrm{ZrO}_{2}\left(8 \mathrm{wt} \% \mathrm{Y}_{2} \mathrm{O}_{3}\right)$ of mixed phase composition [monoclinic (m) and tetragonal $(\mathrm{t})$ ]. Laser excitation with $\lambda_{0}=1.064 \mu \mathrm{m}$. Underlined frequencies are those for the peaks of luminescence bands, given here as Raman shifts.

the exciting line is blocked out by the instrument to prevent overloading the detector by the intense Rayleigh radiation. The Raman signatures on the Stokes side indicate that this material is mostly of the monoclinic phase (see Fig. 11, bottom). From the composition of this material as determined by $\mathrm{x}$-ray analysis, secondary ion mass spectrometry and the Raman microprobe we have some idea that these kinds of luminescence emission, typical of many of these commercial powders, originate from the presence of trace concentrations of rare-earth ion impurities that are introduced through the presence of yttrium and hafnium (via $\mathrm{Zr}$ ). In this way one is able to attribute the Stokes and antiStokes luminescence bands to some of these components.

Sandia National Laboratories is also involved in some aspects of these zirconia systems, and 
they are set up to produce the thermal barrier coatings employing the latest technologies in plasma-spray coating. From Sandia Labs we have received the respective zirconia coatings prepared from a set of Metec \#95086G powders, examining any particle dependence on the phase make-up of the resultant coating. The microRaman spectrum of the Sandia Labs zirconia coating, prepared from the Metec powder, as received (i.e., not size-segregated), is shown in Fig. 16. For the coating also, the predominant features of both the Stokes and anti-Stokes regions are the intense luminescence bands, many of which are as sharp and narrow as Raman lines. In the spectral region $\left(100 \mathrm{~cm}^{-1}\right.$ to $\sim 700 \mathrm{~cm}^{-1}$ ) where one looks for the expected

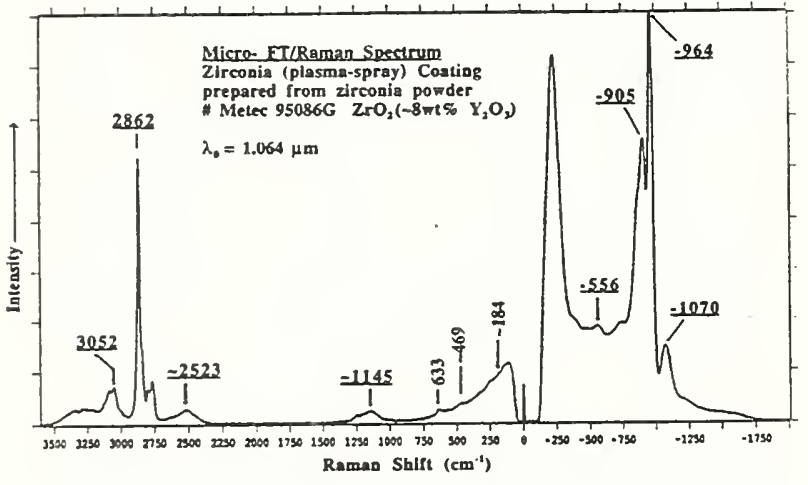

Fig. 16. Micro-FT-Raman spectrum of the (plasmaspray) zirconia coating prepared from Metec powder \#95086G. Coating produced by Sandia National Laboratories. Underlined frequencies are those for the peaks of luminescence bands, given here as Raman shifts.

$\mathrm{ZrO}_{2}$ Raman emissions, one observes a downsloping luminescent background upon which just barely the residual peaks of some Raman bands can be discerned. A closer look shows these faint, obscured bands to be assignable to a mixed tetragonal/monoclinic composition, but nothing more can be inferred.

In our recent work, we have attempted to correlate the micro-Raman results on single zirconia particles with the results from microfocus $\mathrm{x}$-ray diffraction (micro-XRD). In the application of the latter technique, we use a commercial micro-diffractometer with a position- sensitive detector on a rotating anode $\mathrm{x}$-ray generator. The sample goniometer moves the microsample through a large range of angles with respect to the focused beam, provided by a
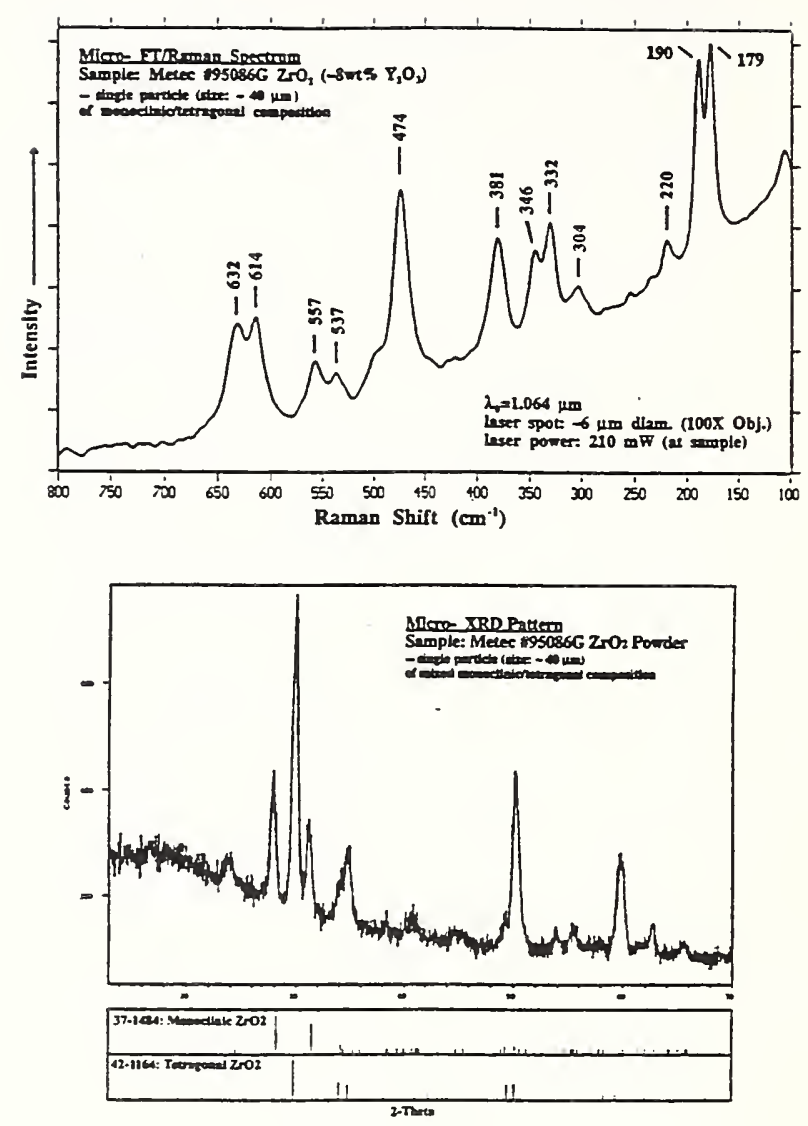

Fig. 17. Micro-FT-Raman spectrum (top) and microXRD patter (bottom) of Metec zirconia powder \#95086G obtained from the same single particle mounted for dual microanalysis. The two structure techniques indicate a mixed monoclinic-tetragonal composition.

$\sim 30 \mu \mathrm{m}$ beam collimator. Typically, single particles of the zirconia powder are mounted at the end of a glass fiber. In this same sample mount, the particle can also be taken to the stage of the FT-Raman microscope. A result from this correlative approach is shown in Fig. 17. It is the result of the two complementary measurements carried out on the same single microparticle picked from the bulk sample of Metec powder \#95086G. For this particle, a mixed monoclinic/tetragonal composition is indicated but can not be quantified by either technique. The lower portion of the micro-XRD pattern shows the standard XRD patterns from the JCPDS file. Similar results have been 
obtained for other, randomly picked microparticles analyzed on both instruments in the same fashion. Overall, a phase heterogeneity is indicated for this powder, on a particle-toparticle basis, that is not evident from bulk sample analysis. The various particles analyzed by both techniques possess varying $\mathrm{m} / \mathrm{t}$ ratios, and very few are found to be monophasic, that is either monoclinic or tetragonal. The matter of any possible cubic phase constituents in this powder sample is not resolved by these micromeasurements.

The present results of this work can be summarized as follows. We have analyzed various non-stabilized zirconias and several yttria-stabilized zirconia powders, to gain information on phase composition. Among these are a number of commercial powders and coatings that have come under investigation as candidate reference materials, in particular Metco-P and Metec powders. The Raman and luminescence spectra show that "clean" zirconia powders exhibit no laser-excited luminescence. However, in contrast to this, commercial zirconia powders and coatings show strong luminescence in the visible, but less so in the near-IR excited Raman spectra. Furthermore, monoclinic, tetragonal, and cubic phases are distinguishable by their Raman spectra, but they can not be quantified. Source powders and coatings all show appreciable luminescence interferences, yet major phase components can be inferred. Size-segregated Metec powders show a fair degree of homogeneity of phase composition. This characteristic suggests that these powders may be suited to serve as a "Standard Reference Material."

\section{Summary}

In this presentation we have provided an overview of the analytical capabilities of near-IR excited FT-Raman microspectroscopy applied to two areas of materials science that are currently under intense scrutiny by many research groups worldwide. In the applications area of CVD diamond, NIR Raman spectroscopy has furnished interesting new results that lead to more sensitive detection of non-diamond carbon impurities in synthetic polycrystalline diamond materials of high quality. Here the advantage results from resonance enhancement effects for $\mathrm{sp}^{2}$-bonded carbon. In the second area of investigation, the application to the zirconia ceramic system for phase identification, the principal advantage of going to near infrared excitation lies in the suppression, not the total elimination, of the luminescence interference problem, so common for real-world materials systems of this type. With respect to the study of both systems, however, the micro-Raman approach cannot furnish the kinds of quantitative results that are needed to fully exploit the analytical information that is gained. Fortunately, in this present study, the absolute Raman scattering intensities of the "analyte" (carbon/diamond and zirconia) are sufficiently favorable to furnish "signal" levels that cause microsampling to be an attractive approach, despite the intrinsically lower Raman intensity that results from long wavelength (NIR) excitation. It is expected that the continued entry of FT-Raman into other, diverse areas of materials science will make further strides, thereby gaining progressive acceptance by the analyst willing to accept the setbacks and shortcomings whenever they occur in the analytical game plan.

\section{Acknowledgments}

The work on CVD diamond was carried out through informal collaboration with my colleagues in the Ceramics Division of NIST, where I thank Edward Farabaugh, Albert Feldman and Lawrence H. Robins for providing samples and engaging in useful discussions. Appreciation is expressed to Crystallume, Norton Diamond Film, and the Raytheon Research Division for the supply of CVD diamond research specimens. The research focused on the zirconia ceramic system was greatly aided by the help of my NIST colleagues Eric B. Steel and Jennifer R. Verkouteren, who agree that there is more to do. Also, it has been a pleasure to have had Luz-Elena Narváez, from the National Standards Institute in Mexico, Centro Nacional de Metrologia (CENAM), participate in the Raman measurements of the zirconia system, while she was a guest scientist at NIST, during 1995-1996. 


\section{References}

1. G.J. Rosasco, E.S. Etz, and W.A. Cassatt, Appl. Spectrosc. 29, 396-404 (175).

2. G.J. Rosasco, "Raman Microprobe Spectroscopy", Chap. 4 in Advances in Infrared and Raman Spectroscopy, R.J.H. Clark and R.E. Hester, Eds. (Heyden \& Son Ltd., London, 1980), vol. 7, pp. 223-282.

3. See the discussions given in Raman Microscopy: Developments and Applications, G. Turrell and J. Corset, Eds. (Academic Press Ltd., London, 1996).

4. See, for example, the reviews given in Fourier Transform Raman Spectroscopy: From Concept to Experiment, B. Chase and J.F. Rabolt, Eds. (Academic Press, 1994), and references cited therein.

5. Y. Wang, D.C. Alsmeyer, and R.L. McCreery, Chem. Mater. 1990, 557-563.

6. D.S. Knight and W.B. White, J. Mater. Res. 4, 385-393 (1989).
7. E.S. Etz, in Microbeam Analysis-1994 (VCH Publishers, Inc., New York, 1994) p. $71 \mathrm{ff}$.

8. J. Wagner, C. Wild, and P. Koidl, Appl. Phys. Lett. 59, 779-781 (1991).

9. E. Anastassakis, B. Papanicolaou, I.M. Asher, J. Phys. Chem. Solids 36, 667 (1975).

10. C.H. Perry, F. Lu, D.W. Liu, and B. Alzyab, J. Raman Spectrosc. 21, 577-584 (1990).

11. C.M. Foster, G.R. Bai, J.C. Parker, and M.N. Ali, J. Mater. Res. 8, 1977-1982 (1993).

12. E.S. Etz and E.B. Steel, in Microbeam Analysis-1995 (VCH Publishers, Inc., New York, 1995) p. $127 \mathrm{ff}$.

13. M. Bowden, G.D. Dickson, D.J. Gardiner, and D.J. Wood, Appl. Spectrosc.44, 16791684 (1990).

\section{Discussion}

Q What is the low wavenumber cut off in the near IR Raman instrument?

A About 80 to $110 \mathrm{~cm}^{-1}$, depending on the scattering from the sample.

Q I am just wondering if CARS is helpful for avoiding luminescence.

A I have not tried this experiment to see whether the luminescence would be reduced in this way though I believe that even with the CARS technique the luminescence problem will persist. In general, CARS requires a transparent medium which is not the case with the materials studied here. Nevertheless, even with transparent materials, if the medium fluoresces interference between the probe and pump can be significant, making signal generation difficult. Measurements with weakly fluorescent materials have been done, but often with the technique of inverse Raman spectroscopy.

Q The luminescence will be there, but because in CARS the beam is very narrow and directed, the incoherent luminescence will contribute an insignificant amount to the signal.

A Yes, indeed. But I am not aware of anyone having looked into this.

Q Have any time resolved experiments been done to deal with the fluorescence/luminescence problem?

A We have not done any such experiments. Such gated detection experiments have been done in the past, but they have not led to practical solutions of the fluorescence interference problem.

Q There is another interesting technique, one in which the exciting line is frequency modulated. The Raman signal then follows this modulation whereas the fluorescence does not. By locking the Raman detection to the modulation frequency of the exciting line one then records only the Raman signal but not the fluorescence/luminescence.

A Yes, this would be a very good technique. 


\title{
Structure and Spectroscopy of Cyclosilicate Minerals
}

\author{
M.I. Bell \\ Condensed Matter and Radiation Sciences Division \\ Naval Research Laboratory \\ Washington, DC 20375
}

and

\author{
D.A. McKeown \\ Department of Chemistry \\ Howard University \\ Washington, DC 20059
}

\begin{abstract}
The results of a series of Raman scattering experiments and lattice dynamics calculations on cyclosilicate minerals are reviewed. Computational methods appropriate to very large unit cells are discussed, and the fitting of complex spectra is described. Particular attention is directed to trends observed as a function of silicate ring size and geometry. The findings support models of the vibrational spectra of silica glasses in which sharp, so-called "defect" lines are attributed to decoupled breathing modes of particular types of silicate rings.
\end{abstract}

\section{INTRODUCTION}

The basic idea that underlies the work described in the following is a suggestion made by $D$. McKeown several years ago, namely to use naturally-occuring cyclosilicate minerals as models for the vibrational properties of rings in silicate glasses. This idea was motivated by the following consideration. It is widely known in glass technologies, certainly in silicate glass technology, that one can substitute elements such as boron, phosphorous, and aluminum for the silicon in glass, and these generally substitute in the continuous random network of the silica. We may also introduce modifiers such as sodium, potassium, strontium, and other elements, and these produce fairly dramatic changes in thermal properties, chemical reactivity, refractive index, and so on. These changes are frequently attributed, in the "conventional wisdom," to changes in ring statistics, that is, the relative numbers of rings of various sizes and geometries in the glass. Elsewhere, silicate minerals of course play an important role in geophysics and geochemistry. Many of them are structurally complex, and their phases vary with temperature and pressure. In the environmental area, clays are known to store and transport environmental contaminants. Zeolites play a central role in catalysis. In semiconductor device fabrication, thermally grown $\mathrm{SiO}_{2}$ and its interface with silicon play important roles in the technology. In view of these facts it is not necessary to belabor the reasons for being interested in the properties of silicate glasses or minerals, or the potential importance of the relationship between ring statistics and physical properties.

In the time and space allowed here the focus will be on basic concepts and motivation. A specific, somewhat narrow technical problem will be discussed which serves to illustrate the benefits of doing Raman spectroscopy, together with relatively simple, but nevertheless realistic lattice dynamic calculations of solids. Since the experimental methods used in this work are standard, there will be no discussion of them here. Instead, the computational methods used will be briefly discussed. Even though they are not what is considered "state-of-the-art", special tools had to be developed for the problems at hand. Rather than giving a catalog of all the results that we have obtained, we will focus on those that lead most directly to the conclusions about the problem that we are investigating. 


\section{Classes of Silicate Minerals}

Mineralogists have developed a useful taxonomy of the silicate minerals, all of which have structures based on the $\mathrm{SiO}_{4}$ tetrahedron. The cyclosilicates to which Dr. McKeown drew attention contain one type of isolated silicate ring. The tetrahedra share corners linked together so that a typical unit is an $\mathrm{SiO}_{3}$ group repeated $n$ times to form an $n$-membered ring. The other varieties of silicates are phyllosilicates (sheets of connected rings, typically clays and micas) and tectosilicates (three-dimensional frameworks such as quartz and its polymorphs, zeolites, and a great many other naturally occurring minerals). The generally accepted model for a silicate glass is a continuous random network of linked $\mathrm{SiO}_{4}$ tetrahedra. Like any other classification, this one has its shortcomings: there are some minerals that do not fall cleanly into any one of these categories.

\section{Cyclosilicates}

A preliminary search of the literature revealed a substantial number of cyclisilicate structures. Fig. Iillustrates the isolated rings found in two
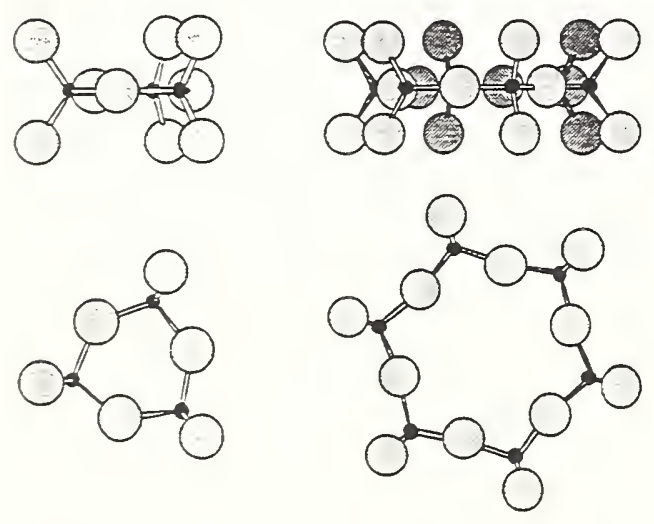

BENITOITE

$$
\begin{gathered}
\text { BERYL } \\
a[S i-O(b r)]=1.59 \AA
\end{gathered}
$$

Fig. 1. Ring structure of two cyclosilicates, benitoite $\mathrm{BaTiSi}_{3} \mathrm{O}_{9}$ and beryl $\mathrm{Be}_{3} \mathrm{Al}_{2} \mathrm{Si}_{6} \mathrm{O}_{18}$

minerals. Benitoite $\mathrm{BaTiSi}_{3} \mathrm{O}_{9}$ contains a threemembered ring which is planar, and beryl $\mathrm{Be}_{3} \mathrm{Al}_{2} \mathrm{Si}_{6} \mathrm{O}_{18}$, a semiprecious stone, has aplanar six-membered ring. The silicon-oxygen distance for the bridging oxygen that connects the silicons in the ring is given in the figure. It ranges in these two minerals from roughly the largest to approximately thje smallest value found in the cyclosilicate family. So, in a sense, these structures represent extremes in the behavior of planar rings in naturally-occurring cyclosilicates.

Though we have identified in the literature many candidates for this sort of study, we list in Table I only those crystals that we have actually studied in detail.

The minerals listed in Table I contain three-, four-, and six-membered rings. Most exhibit the

Table 1. Cyclosilicate minerals examined in this study.

3-Membered Rings 4-Membered Rings 6-Membered Rings

$\begin{array}{ccc}\text { Benitoite(28) } & \text { Gillespite(32) } & \text { Beryl(58) } \\ \text { BaTiSi }_{3} \mathrm{O}_{9} & \mathrm{BaFeSi}_{4} & \mathrm{Be}_{3} \mathrm{Al}_{2} \mathrm{Si}_{6} \mathrm{O}_{18}\end{array}$

Wadeite(30) "Ba-Cu silicate"(20) Bazzite(58)

$\mathrm{K}_{2} \mathrm{ZrSi}_{3} \mathrm{O}_{9} \quad \mathrm{BaCuSi}_{2} \mathrm{O}_{6} \quad \mathrm{Be}_{3} \mathrm{Sc}_{2} \mathrm{Si}_{6} \mathrm{O}_{18}$

Dioptase(48)

$\mathrm{Cu}_{6} \mathrm{Si}_{6} \mathrm{O}_{18} \cdot 6 \mathrm{H}_{2} \mathrm{O}$

three-to-one ratio of oxygen to silicon typical of cyclosilicates, but naturally, there is an exception: gillespite $\mathrm{BaFeSi}_{4} \mathrm{O}_{10}$ has additional $\mathrm{Si}-\mathrm{O}-\mathrm{Si}$ bridges linking the rings which change the Si:O ratio and complicate the situation slightly. We began our study using benitoite [13], gillespite and beryl [4] as representative of three-, four, and six-membered rings, respectively. We were soon introduced, however, to a synthetic "barium copper silicate" $\mathrm{BaCuSi}_{2} \mathrm{O}_{6}$ produced at the Geophysical Laboratory which contains a four-membered ring [8]. Since good Raman scattering data were already available, we diverted our attention to this material [7]. We have very recently completed a study of gillespite which will b submitted for publication shortly. Table I indicates in parentheses the number of atoms in the conventional unit cell. These values demonstrate that the lattice dynamics calculations are far from trivial. A brute-force approach to the calculation would require the diagonalization of a matrix of dimension equal to three times these values (i.e., equal to the number of degrees of freedom in the unit cell). 


\section{Benitoite $\mathrm{BaTiSi}_{3} \mathrm{O}$,}

We began by comparing the Raman spectra of benitoite (which contains three-membered rings) and beryl (containing six-membered rings) in the spectral region where we might expect the ring vibrations to occur. The results are shown in Fig. 2. The spectra contain scattering by both

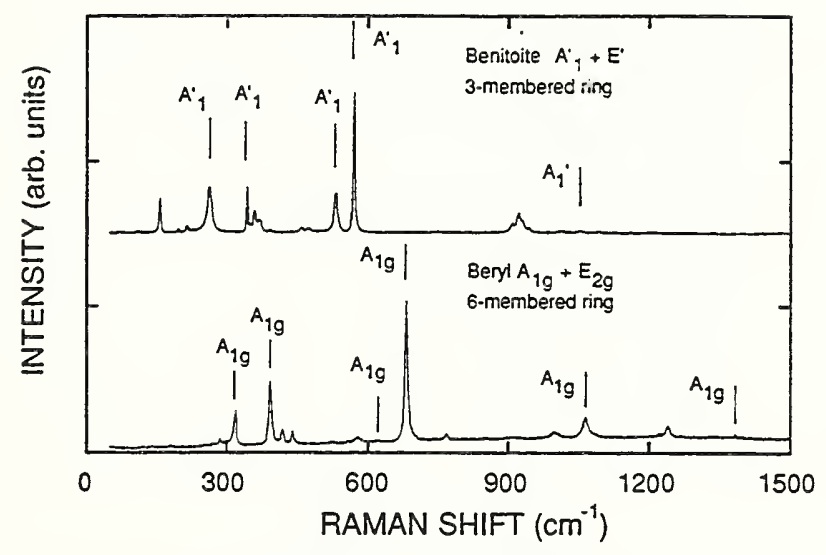

Fig. 2. Comparison of the Raman spectra of benitoite and beryl.

the fully symmetric and doubly degenerate modes. We made additional measurements in other scattering geometries in order to determine which of these lines are in fact the fully symmetric A modes. Not surprisingly, the symmetric modes have a striking similarity. There is a good correspondence between sets of spectral features which are very similar in the two crystals but distinctly shifted to higher frequency in the six-membered ring, reflecting the strain (compression) of forcing six units rather than three into a planar ring. Qualitatively, this is the behavior we expected, but it was clear that a quantitative description of these effects would require modeling of the lattice dynamics.

Now at much the same time, we became aware of work $[9,10]$ on the interpretation of the spectra of glasses that altered our thinking about this project. Fig. 3 shows the polarized spectrum of amorphous $\mathrm{SiO}_{2}$ (fused silica). The conventional wisdom when we started this project was that the broad features in the spectrum reflect the continuous random network and thus could, in principle, provide information about the ring statistics. The sharp features at $495 \mathrm{~cm}^{-1}$ and $606 \mathrm{~cm}^{-1}$, the so-called "defect lines", $D_{1}$ and $D_{2}$, respectively, were attributed

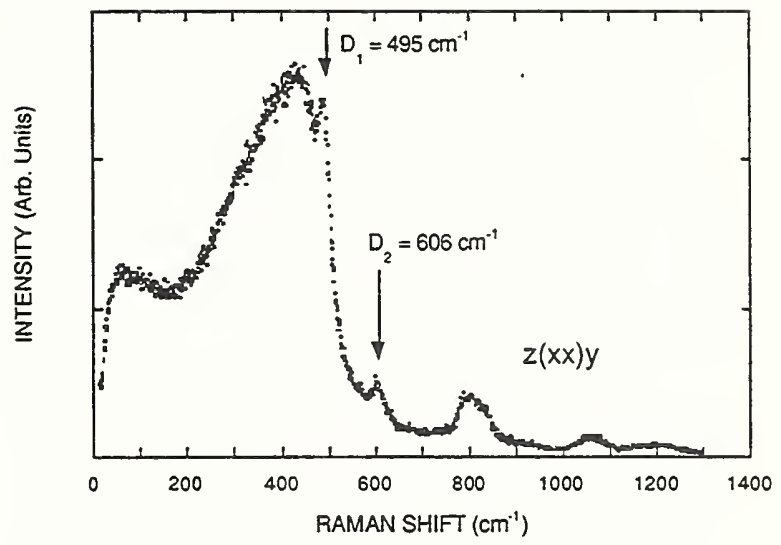

Fig. 3. Polarized Raman spectrum of amorphous $\mathrm{SiO}_{2}$.

to point defects, such as dangling bonds or wrong bonds. In this interpretation, the frequency or intensity of these features does not provide much structural information about the glass.

The work of Galeener, Elliott, and their colleagues $[9,10]$ suggested a different view: that these sharp features represent modes which are localized on silicate rings and decoupled from the surrounding network. In the absence of any broadening due to coupling to the disordered network, such modes would remain narrow in frequency and localized in space. Fig. 4 illustrates how such a mode could occur on a

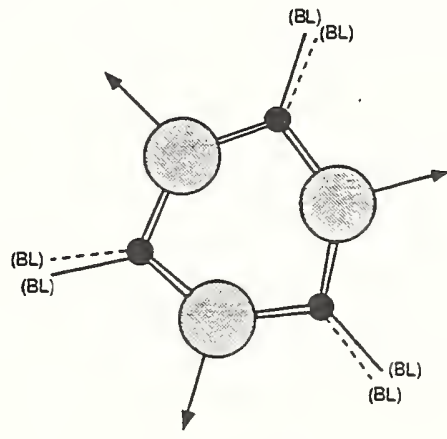

$(B L)=$ Bethe Lattice

Fig. 4. Decoupled bridging-oxygen breathing mode of a planar three-membered ring, proposed for the $D_{2}$ defect line.

planar three membered ring. The bridging oxygens oscillate, but for a certain ratio between 
the bond-stretching and bond-bending forces on the silicon atoms, the silicons and non-bridging oxygens remain stationary. This concept of a decoupled bridging-oxygen breathing mode was used in a simple computational model in which each non-bridging oxygen is replaced by a Bethe lattice (an infinite branched tree), representing the continuous random network in which the ring is embedded.

The geometry and atomic force constants of the Bethe lattice (denoted by BL in Fig. 4) can be chosen so as to generate the broad bands sen in the spectrum of Fig. 3. The ratio of bondstretching and bond-bending forces on the silicon which is required for the existence of a decoupled mode on the planar three-membered ring is not far from that observed in many silicates, and the calculated frequency is close to that of the $D_{2}$ line at $606 \mathrm{~cm}^{-1}$. (The force constant ratio is not particularly critical. Variations of about $\pm 50 \%$ do not shift the decoupled mode frequency by more than $20 \mathrm{~cm}^{-1}$, the observed width of the $D_{2}$ line.) A similar but slightly more complex analysis of the $D_{1}$ mode at $495 \mathrm{~cm}^{-1}$ suggests that it is due to a slightly puckered (nonplanar) four-membered ring [10].

Galeener and his coworkers [9] also suggested that the decoupled modes ought to occur in crystals and matrices. We therefore began our lattice dynamics calculations with the goal of determining whether such modes do in fact exist in crystalline silicates in addition to explaining quantitatively the effects of ring size on spectar such as sen in Fig. 2. From the beginning, we realized not only that we would need factor analysis but that the correlation method of Fateley and his coworkers [11] would be particularly valuable. This method allows one to determine whether or not motion of a particular atom contributes to vibrational modes of a given species. This information can provide valuable insight into the nature of certain modes, even without a direct calculation of the atomic motion eigenvector. We employed a valence force potential (VFP) model, containing only nearestneighbor bond-stretching and (three-body) bondbending interactions involving an atom and two of its nearest neighbors. One can think of this VFP as a "ball and spring" model. It produces a large set of coupled linear equations (the secular equations), and the solution simply involves diagonalizing the dynamical matrix.
We made rapid progress once we had developed efficient methods for block diagoalization. The dynamical matrix can be broken up into submatrices, one corresponding to each of the symmetry species. This not only reduces the size of the problem but also permits independent fitting of the experimental data species by species. One may also chose which observed modes are assigned to a given species and which are used or excluded in a fitting. The results can be quite sensitive to the choice of targets for the fitting procedure, so this flexibility turns out to be very important for making progress with the problem. Of course, we also needed to save and display the eigenvectors in order to be able to identify any decoupled modes.

Fig. 5 shows the full crystal structure of benitoite. The unit cell is relativeley large, containing 28 atoms including two $\mathrm{Si}_{3} \mathrm{O}_{9}$ rings.

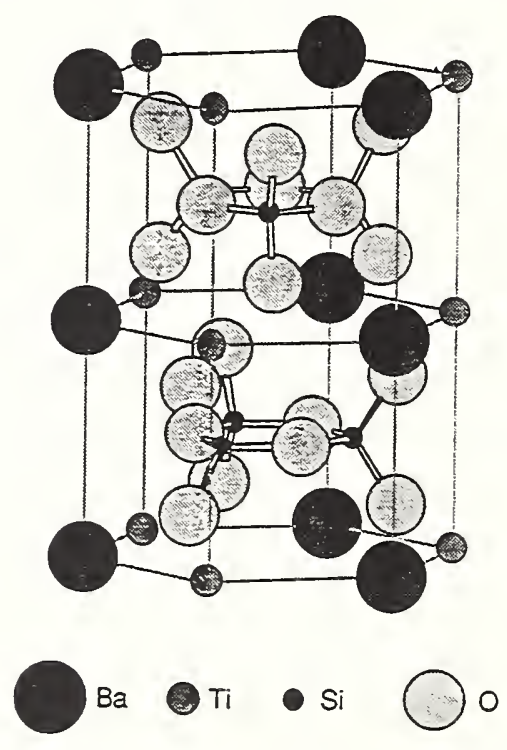

Fig. 5. The unit cell of benitoite $\mathrm{BaTiSi}_{3} \mathrm{O}_{9}$.

The factor analysis for benitoite yields 35 Raman-active modes, 23 infrared-active modes, and 14 silent ones. This is a fairly large problem, and benitoite is one of the simplest of the cyclisilicates that we examined. The calculations were performed on a workstation (microVAX) because of the limitations (since overcome) of memory size and memory management technology available for desktop computers.

We were able to identify 34 of the 35 Ramanactive modes in our data. We chose not to fit all of them because the $E^{\prime}$ modes are simultaneously 
Raman-and indrared-active and therefore can have longitudinal-transverse splittings. Since our model contains only short range interactions (i.e., no Coulomb forces), we did not really know how to treat these experimental values. Our experiments indicated that the LO-TO splittings were too small to observe (we were never actually able to resolve any slpittings), but out of caution we used only infrared-inactive modes in the fitting. The force constants were obtained by fitting 19 observed frequencies (the $A_{1}^{\prime}$ and $E^{\prime \prime}$ modes) produced good agrement with the experiment $\left(\approx 10 \mathrm{~cm}^{-1} \mathrm{rms}\right.$ error $)$. The measured spectra and predicted mode frequencies are shown in Fig. 6. When we calculated the $E^{\prime}$ mode frequencies, none of which were used in the fitting, the agreement

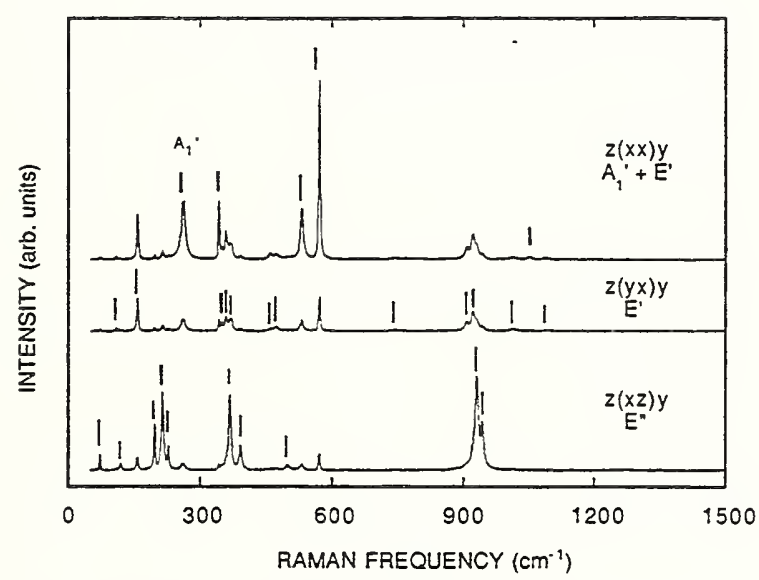

Fig. 6. Measured Raman spectra and mode symmetry assignments for benitoite $\mathrm{BaTiSi}_{3} \mathrm{O}_{9}$.

was again quite good. Overall, we obtained a satisfactory account of the vibrational spectrum of benitoite.

A striking aspect of the results is that the doubly-degenerate modes have s strong tendency to occur in pairs (see Fig 6). This results from the fact that there are two rings in the unit cell. If the rings were completely decoupled from one another, every mode localized on a ring would belong to a degenerate pair. A given pattern of developments on one ring would yield exactly the same frequency as the corresponding pattern of displacements on the other ring. Because the rings are coupled to one another through interactions of the non-bridging oxygens, the symmetery-induced (Davidov) pair is split. We can determine the magnitude of the coupling between the rings quantitatively by a procedure we describe shortly.

\section{Ba-Cu silicate $\mathrm{BaCuSi}_{2} \mathrm{O}_{6}$}

The rest of this discussion will draw from our recent unpublished work on $\mathrm{BaCuSi}_{2} \mathrm{O}_{6}$. Just as in the case of benitoite, we obtained a set of force constants that give a reasonable good fit to the spectrum. We calculated the effect of turning off the interactions between the rings by eliminating the interactions of the non-bridging oxygens with the other atoms in the cell, in this case the barium-oxygen and copper-oxygen interactions. If these interactions are simply reduced to zero, it can be impossible to determine which mode of the isolated ring corresponds to a given mode the fully interacting crystal. To avoid this difficulty, we turmed the interactions off gradually, introducing the parameter $\alpha$ which we use to scale the inter-ring couplings. (The force constants have their full experimental values for $\alpha=1$ and vanish for $\alpha=0$.) By this method, we obtained diagrams similar to Fig. 7, where we can clearly identify the low-frequency modes associated with motions of the barium or copper atoms. The frequencies of these modes go to zero as we

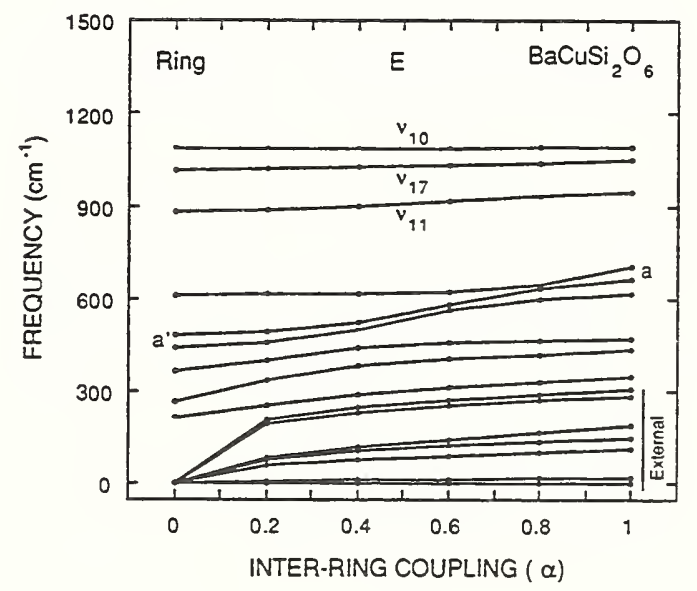

Fig. 7. Raman frequencies as a function of inter-ring coupling $\alpha$ for the $E$ modes of $\mathrm{BaCuSi}_{2} \mathrm{O}_{6}$.

reduce the force constants associated with motion of these atoms; the modes are essentially external to the ring.

Of equal importance is the behavior of the external modes as the interactions increase and 
their frequencies approach those of the ring modes. These mode crossings can lead to an exchange of character between the external and ring modes and to a complex pattern in the coupling diagram which is described in detail in Ref. 4.

Returning to the basic concpt of a decoupled bridging-oxygen breathing mode, we found that both the four-membered ring in $\mathrm{BaCuSi}_{2} \mathrm{O}_{6}$ and the three-membered ring in benitoite exhibit such modes. $\mathrm{BaCuSi}_{2} \mathrm{O}_{6}$ contains a puckered, nonplanar, four-membered ring, and the decoupled $A_{1}$ mode shown in Fig. 8 occurs at $511 \mathrm{~cm}^{-1}$ for the crystal and shifts slightly to 515 $\mathrm{cm}^{-1}$ when we turn off the interactions. Even though the ring is puckered, and the breathing motions are out of the plane, one still obtains a decoupled mode.
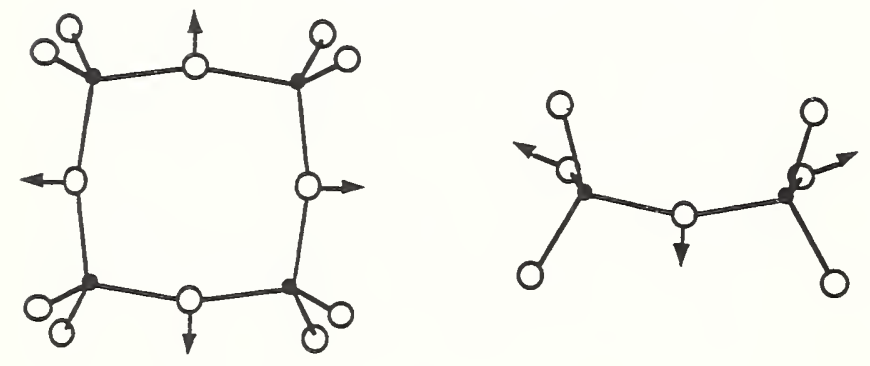

Fig. 8. Decoupled bridging-oxygen breathing mode in $\mathrm{BaCuSi}_{2} \mathrm{O}_{6}$.

Table II presents a summary of our results with respect to decoupled modes. Wadeite, another

Table II. Frequencies (in $\mathrm{cm}^{-1}$ ) of the decoupled bridging-oxygen breathing modes in the cyclosilicates studied.

$\begin{array}{ccc} & \text { 3-Rings } & \\ \mathrm{a}-\mathrm{SiO}_{2}\left(D_{2}\right) & \text { Benitoite } & \text { Wadeite* } \\ 606 & 633 & 625,629 \\ & \text { 4-Rings } & \\ & \mathrm{BaCuSi}_{2} \mathrm{O}_{6} & \mathrm{BaCuSi}_{2} \mathrm{O}_{6} * \\ \mathrm{a}-\mathrm{SiO}_{2}\left(D_{1}\right) & (I 4 \mathrm{~m} 2) & \left(\mathrm{I} 4 / \mathrm{mmm}^{*}\right) \\ 495 & 511 & 526\end{array}$

*Not completely decoupled three-membered ring silicate, has a decoupled mode whose frequency is almost the same as that benitoite and not far at all from the $D_{2}$ defect in silica glass. In the case of the wadeite structure, the mode is not clearly decoupled.; there is some motion of the silicon and non-bridging oxygen. $\mathrm{BaCuSi}_{2} \mathrm{O}_{6}$ has acentric $(14 \mathrm{~m} 2)$ symmetry and a puckered four-membered ring; a hypothetical centric $(14 / \mathrm{mmm})$ structure has been proposed, where the rings are planar [8]. Our calculations suggest that the frequency of the decoupled mode for the acentric structure is very close to the $D_{1}$ defect in the glass. A similar mode (not completely decoupled) for the centric structure has a slightly higher frequency.

We were also able to make some reasonable estimates of the linewidth one might expect to find in a decoupled mode if it is embedded in a random environment like a glass. Fig. 9 shows the behavior of the bridging-oxygen breathing mode in $\mathrm{BaCuSi}_{2} \mathrm{O}_{6}$, denoted $\mathrm{O}(2)$ br. In the figure, as all its external interactions are reduced.

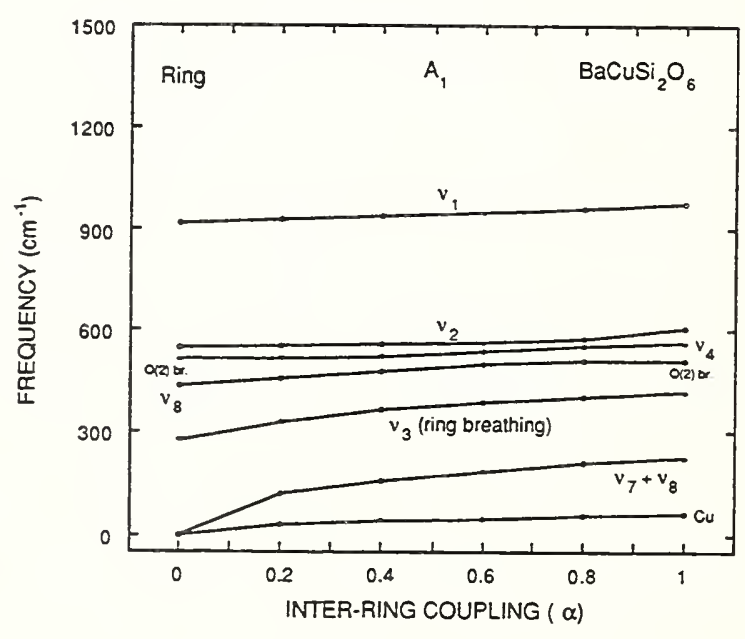

Fig. 9. Raman frequencies as a function of inter-ring coupling $\alpha$ for the $A_{1}$ modes of $\mathrm{BaCuSi}_{2} \mathrm{O}_{6}$.

Because the mode is not completely decoupled, it interacts slightly with another mode on the ring and the two exchange character. In this sense the mode which is primarily an $O(2)$ breathing mode at $\alpha=0$ is not the decoupled mode actually observed in the crystal. This explains why the frequency of the decoupled mode shifts upward rather than downward as the 
interactions are reduced. (The effect is small, however. The entire shift is only about $4 \mathrm{~cm}^{-1}$.)

Similar coupling calculation were performed to estimate the effect of external perturbations on the decoupled modes in each of the crystals that we studied. If we regard the change in frequency between $\alpha=0$ and $\alpha=1$ as an estimate of the width of each mode in a random environment, we obtain the linewidths shown in Table III. The widths are very small in benitoite $\left(4 \mathrm{~cm}^{-1}\right)$ and $\mathrm{BaCuSi}_{2} \mathrm{O}_{6}\left(4-10 \mathrm{~cm}^{-1}\right.$, depending on the structure). Even in wadeite, where the decoupling is incomplete, the estimated linewidth of $40 \mathrm{~cm}^{-1}$ is not significantly greater

Table III. Linewidths (in $\mathrm{cm}^{-1}$ ) of the decoupled bridging-oxygen breathing modes in the cyclosilicates studied.

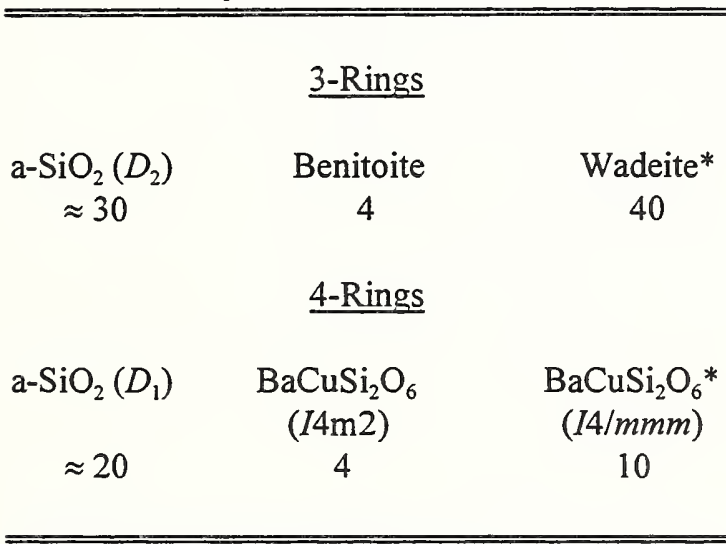

*Not completely decoupled.

than the $\approx 30 \mathrm{~cm}^{-1}$ observed for the $D_{2}$ line in glasses.

\section{Conclusions and Outlook}

In summary, our work strongly suggests that there is physical truth in the mode-decoupling hypothesis for glasses. Decoupled bridgingoxygen breathing modes occur in natural cyclosilicate materials, as demonstrated by our experimentally verified lattice dynamics models. These modes are analog of the local defect modes proposed for glasses. While there are interacting structural and dynamic requirements which must be met in order for these modes to occur, we find that complete decoupling is not required in order to produce narrow lines. All of the materials we have studied have decoupled or nearly decoupled modes which when embedded in a random environment could produce bands no wider than those observed in glasses. The exact nature of the incomplete decoupling in wadeite and some of the other minerals we have studied is not fully understood, however, and further work is needed to explore the interaction of these modes with their environments.

The mode-coupling calculations have also enabled us to identify the nearly degenerate Davydov pairs and their splittings. Both wadeite and $\mathrm{BaCuSi}_{2} \mathrm{O}_{6}$ have had both centric and acentric structures proposed. We have resolved those ambiguities and found that both minerals have acentric structures.

In terms of methodology, we have shown that complex crystal structures (100 or more degrees of freedom) can be modeled fairly simply and accurately, but certain computational tools are needed. One needs the ability to block diagonalize and fit individual symmetry species. The mode coupling diagrams of Figs. 7 and 9 are a particularly useful tool. We are now migrating our software back to Windows and the MacOS, because desktop operating systems no longer present serious limitations. Unfortunately, only phenomenological models like the VFP can be used at the present time in this approach. It would be very helpful if first-principles lattice dynamics calculations could be made efficient enough to $b$ used in this context.

We have so far examined only one fourmembered ring silicate. (Gillespite, mentioned above, contains four-membered rings which are linked together in a way which makes its behavior intermediate between that of a cyclosilicate and that of a phyllosilicate.) If we are to make conclusive arguments about decoupled modes, we must study more fourmembered ring structures. We have also observed decoupled modes in six-membered ring silicates, but these occur at frequencies which do not correspond to any structure in the spectra of glasses. We need to understand whether this is because regular six-membered rings simply do not occur in glasses or because they do not meet the geometric or dynamic requirements to generate narrow lines.

We also plan to do some work on other silicate families, layer silicates and even tectosilicates. Initially, we did not think that our computational methods would apply here, but there has been recent success using VFP calculations for quartz and cristobolite, so we will take another look at this possibility. Clays and micas are also 
important for environmental issues, and we plan to determine, both theoretically and experimentally, whether the adsorption of small organic molecules by clays can be detected and studied by Raman spectroscopy.

In the course of the work, we noted a number of trends related to bond lengths, bond angles, and force constants, but to determine the significance of these trends we must know the uncertainties in our force constants. Calculating such error estimates requires yet another generation of repetition of the basic fitting procedure, and we want to do that in at least some selected cases to see whether the observed trend is real, that is, outside the noise of the calculation.

In closing, we hope that by presenting this particular example of the interaction of modeling and experiment in Raman spectroscopy we have demonstrated the value of this approach and perhaps inspired others to address problems which in the past might have been considered to be too complex for spectroscopic analysis.

\section{Acknowledgements}

The pursuit of this project involved a number of people, including three undergraduate students at Howard University (Angela Nobles, George Mulenda Mpoyo, and Michelle Quash) and a former NRL/NRC Postdoctoral Research Associate, Charles Kim, who set up most of the computational machinery used in this work. We have also benefited from the advice of Larry Boyer of NRL. Samples were provided by Russ Hemley of the Geophysical Laboratory at the
Carnegie Institution of Washington and financial support was given by the Office of Naval Research. The authors are grateful to these individuals and institutions for the support rendered.

\section{References}

1. C.C. Kim, M.I. Bell, and D.A. McKeown, Mat Res. Symp. Proc. 291, 515 (1993).

2. C.C. Kim, M.I. Bell, and D.A. McKeown, Phys. Rev. B 47, 7869 (1993).

3. D.A. McKeown, M.I. Bell, and C.C. Kim, Phys. Rev. B 48, 16357 (1993).

4. C.C. Kim, M.I. Bell, and D.A. McKeown, Physica B 205, 193 (1995)

5. D.A. McKeown, M.I. Bell, and C.C. Kim, Phys. Chem. Minerals 22, 137 (1995).

6. D.A. McKwown, A.C. Nobles, and M.I. Bell, Phys. Rev. B 54, 291 (1996).

7. D.A. McKeown and M.I. Bell, "Vibrational analysis of $\mathrm{BaCuSi}_{2} \mathrm{O}_{6}$ and its fourmembered ring," Phys. Rev. B, to be published.

8. L.W. Finger, R.M. Hazen, and R.J. Hemley, Am. Min. 74, 952 (1989).

9. F.L. Galeener, R.A. Barrio, E. Martinez, and R.J. Elliott, Phys. Rev. Lett. 53, 2429 (1984).

10. R.A. Barrio, F.L. Galeener, E. Martinez, and R.J. Elliott, Phys. Rev. B 48, 15672 (1993).

11. W.G. Fateley, F.R. Dollish, N.T. McDevitt, and F.F. Bentley, Infrared and raman Selection Rules for Molecular and Lattice Vibrations: the Correlation Method, (Wiley, New York, 1972).

\section{Discussion}

Q I have a question on the mode coupling as expressed in the dynamical matrix. What is the physics of this? I guess what I would do there is put the coupling strength alpha where the zeros are and make the matrix essentially $2 \times 2$.

A This aspect is discussed in detail in our paper cited as reference 4. The matrix in question is

$$
D=\omega_{0}^{2}\left(\begin{array}{lll}
1 & 0 & \alpha b \\
0 & 1 & \alpha b \\
x b & \alpha b & \alpha
\end{array}\right)
$$

A brief answer to the question is that there really are three modes involved. These matrix elements represent a coupling between the third mode and the other two. 
What is that mode?

A That is an external mode. These are all modes of the same symmetry to begin with. There is no symmetry change as you turn on the interaction $(\alpha>0)$. This is already a block-diagonalized piece of the dynamical matrix. The third mode is external; it has zero frequency in the absence of any interactions (i.e., $\alpha=0$ ) with the other modes. We turn on both the diagonal and off-diagonal parts of the interaction in proportion. It is, however, only a crude way of doing this. In fact, we have thought about other ways of turning these interactions on and off selectively to see what effect they have.

Q Presumably you could have non-bonding interactions in your potential function and do it that way.

A Yes. In fact, for a time we thought that there were some modes which we could stabilize only with non-bonding interactions, which was sort of scary. When you are staring at these structures with so many atoms in the cell and entering the bond interactions one by one it is easy to lose track and leave out interactions. We would like to automate this whole process. Each time we take up a new crystal, you make a man-month worth of mistakes in writing down the potential.

Q You showed a spectrum of a glass (Fig. 3) with the broadened peaks. Are all glasses alike, or is the spectrum representative of just fused quartz or a particular glass?

A Yes, all glasses are more alike than you would wish. That is the difficulty. This is not a new problem: people have tried for a long time to tease out information, or confirmation of their prejudices, about ring statistics from changes in shape or amplitude all throughout that spectrum. There are subtle changes as you modify the glass, even as you change its fictive temperature at the same composition. There are changes, but they are just simply not understood.

Q Is there any remnant of natural crystal quartz?

A No, not so that you would notice. No.

Q There is a strong line in quartz at about $460 \mathrm{~cm}^{-1}$ not very far from your fourmembered ring breathing mode.

A Quartz is basically all three-membered rings, and they are very distorted, irregular and non-planar.

Q I have forgotten where the really strong polarized line of quartz is but it is not much further than your errors from the D1 line.

A Yes, that is a problem. Quartz has a relatively small lattice constant and a relatively rigid structure. It has its three-membered ring modes at much higher frequencies than almost any other silicate. For reasons too complex to discuss here, there is no evidence for three-membered ring modes in glasses, so it is probably misleading to try to read anything from the quartz spectrum into their spectra.

Q You have not even hinted at the practical applications of all this elegant work other than to say that the Army was interested in detecting explosive residues. Is this good, for example, for getting rid of the plutonium stockpile? Is this a matrix for doing these kinds of things?

A Perhaps you are a better salesman than I am. I have not been to the DoE (Department of Energy) lately, but I hear that they are interested in this sort of thing. At least this is what I have been told. 
Q You are not putting things into a silicate cage here, are you?

A We are just scratching the surface here. I do not think we are at the stage where we can say anything about a real glass composition. To be perfectly honest, I think I would be fooling you if I said we could tell which glass is likely to have a smaller coefficient of thermal expansion or chemical resistance, or a particular refractive index or density. But, it is true, that this is where this research leads to. 


\title{
Effects of Doping on the Electronic Raman Spectrum of Cuprate Superconductors
}

\author{
Chris Kendziora \\ Directed Energy Effects Branch, Code 6653 \\ Naval Research Laboratory, Washington DC 20375
}

\begin{abstract}
Raman spectroscopic studies of superconductors can yield information relating to the origins of superconductivity through a measurement of the associated energy gap. This has proven particularly interesting in the case of high $T_{c}$ cuprate superconductors due to the anisotropy in the gap observed in optimally doped crystals. In this paper, we introduce the cuprate superconductors and discuss the experimental concerns in measuring their Raman scattering properties. We discuss how polarization selection can lead to anisotropic $k$-space sensitivity and illustrate this phenomenon with examples from our data. The observations in optimally doped crystals are complimented by studies throughout the phase diagram, where a wide variety of physical phenomena characterize these relatively new materials.
\end{abstract}

\section{THE CUPRATE SUPERCONDUCTORS}

Superconductivity with a record high transition temperature $(40 \mathrm{~K})$ was discovered in a layered perovskite cuprate in 1986. [1] In the 10 years since that time, a host of related materials with even higher superconducting transition temperatures have been synthesized, with the current standard of over $160 \mathrm{~K}$ under high pressure. As the quality of the crystals available for study advances, so too does the understanding which comes from their spectroscopic investigation. In this paper we discuss the study of cuprate superconductors with Raman scattering and illustrate the wide range of observable phenomena with examples from our research.

The cuprates have several distinguishing general features, some of which are thought to affect the origins of superconductivity in these materials. The "parent" compounds are anti-ferromagnetic insulators. When extra carriers are introduced (doping) by either cationic substitution or oxygen intercalation, the resistivity moves through an insulator to metal transition. The metallic phase turns out to be superconducting at low temperatures. A simplistic phase diagram for a typical cuprate is shown in Figure 1, where the critical temperature is observed to fall along a "bell shaped curve" as a function of doping level.

Structurally, the perovskites are layered materials with stacked planes of oxides repeating to form the unit cell. An example of this is shown in Figure 2, where the crystal structures of the three members of the $\mathrm{Bi}$ based cuprate family are depicted. The superconductors have in common a $\mathrm{CuO}_{2}$ plane which accepts charge from the interstitial donor layers. The local (hole or electron type) carrier concentration of these $\mathrm{CuO}_{2}$ planes rises with doping of charge by cationic substitution or oxygen intercalation. In this way, the metallic nature of the crystals is confined to two dimensional planes which are weakly coupled along the $c$-axis. This $2 \mathrm{D}$ property of the normal state influences the superconductivity, and the cuprates have come to be understood as stacked arrays of supercon-

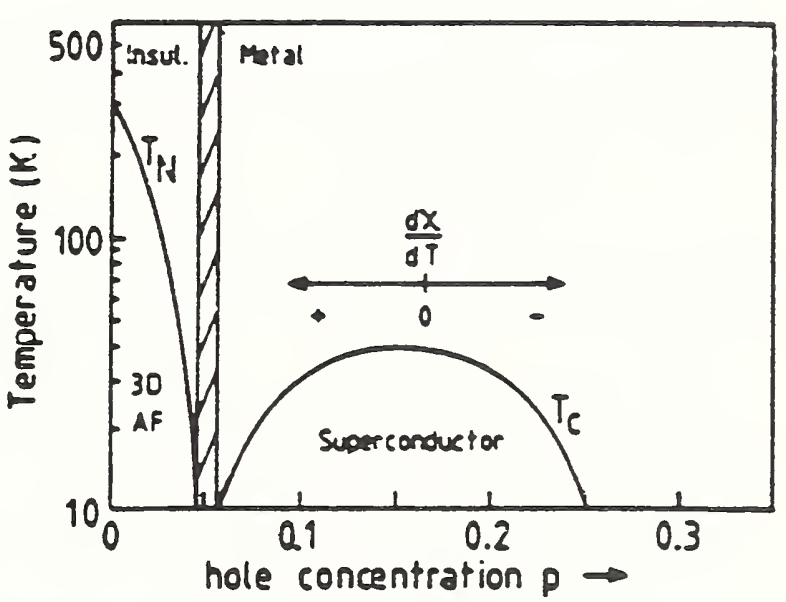

FIG. 1. A simplistic phase diagram for a typical cuprate, where the critical temperature is shown to fall along a "bell shaped curve" as a function of doping level.

ducting tunnel junctions, where the $\mathrm{CuO}_{2}$ derived wavefunctions overlap across the insulating barrier.

\section{RAMAN STUDIES OF CUPRATES}

Raman spectroscopic studies of the cuprates are complicated by some-of their physical features. The materials are black (absorbing), shiny (reflecting) and metallic, which limits the skin depth of light (and thus the range probed by Raman experiments) to roughly 1000 Angstroms. As a consequence, the scattering cross section is very small, resulting in low signal levels and virtually requiring multi-channel detection. Single channel scans utilizing photomultiplier tubes with very low "dark count" rates $(\approx 1$ count/second) can take upwards of 12 hours to measure the region of interest from $20 \mathrm{~cm}^{-1}$ to $1000 \mathrm{~cm}^{-1}$. The highly reflective nature of the crystals, as well as the surface roughness they tend to develop during growth and from sensitivity to humidity, requires the stray light rejection available with triple monochroma- 


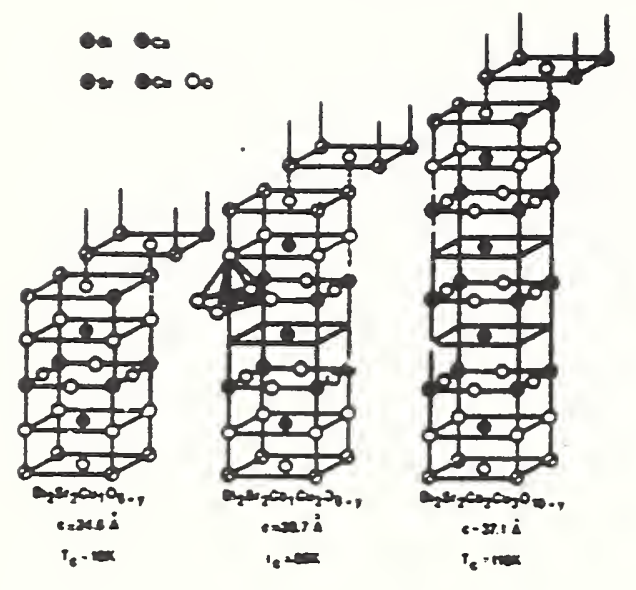

FIG. 2. The crystal structures of the three members of the Bi based cuprate family. These materials are characterized by 1,2 and 3 metallic $\mathrm{CuO}_{2}$ planes separated by insulating interstitial layers.

tors operated in subtractive mode. This rejection is at a premium due to the interest in features which lie so close to the laser line.

A formidable issue in the study of superconductivity in these materials is the degree of heating by the incident laser beam. The two dimensional nature of these materials contributes to a very poor thermal conductivity, which, when combined with the samples' absorption, can lead to large thermal gradients in the vicinity of the laser spot. In this context, the crucial experimental parameter is laser power per area of the spot size. More laser power can be used if the shape of the spot is elongated to a line which can be imaged on the collection slit. Experimentally, the local spot temperature can be measured by comparing the Stokes and Anti-stokes spectra and utilizing the following relation:

$$
I_{A S}(\hbar \omega)=I_{S T}\left(-n_{\omega}\right)\left(\frac{\omega^{I}+\omega}{\omega^{I}-\omega}\right)^{2} \exp \left(\frac{|\hbar \omega|}{k T}\right) .
$$

derived from the Bose thermal occupation factors. An example of this relation is illustrated in Figure 3, where we have scaled the Stokes spectrum by Eq. 1 and re-plotted it along with the anti-Stokes spectrum. The nominal temperature was $10 \mathrm{~K}$, but the fit to the data indicates a local temperature of $130 \mathrm{~K}$ within the laser spot, well above $\mathrm{T}_{c}$ for this material. Depending on several factors, a typical estimate for the extent of laser heating is on the order of $10 \mathrm{~K} / \mathrm{mW}$ for spot sizes in the range of $100 \mu \mathrm{m}$. Thus, low temperature measurements of cuprates are restricted to a range of laser powers constrained from above and below by concerns about laser heating and low signal levels, respectively.

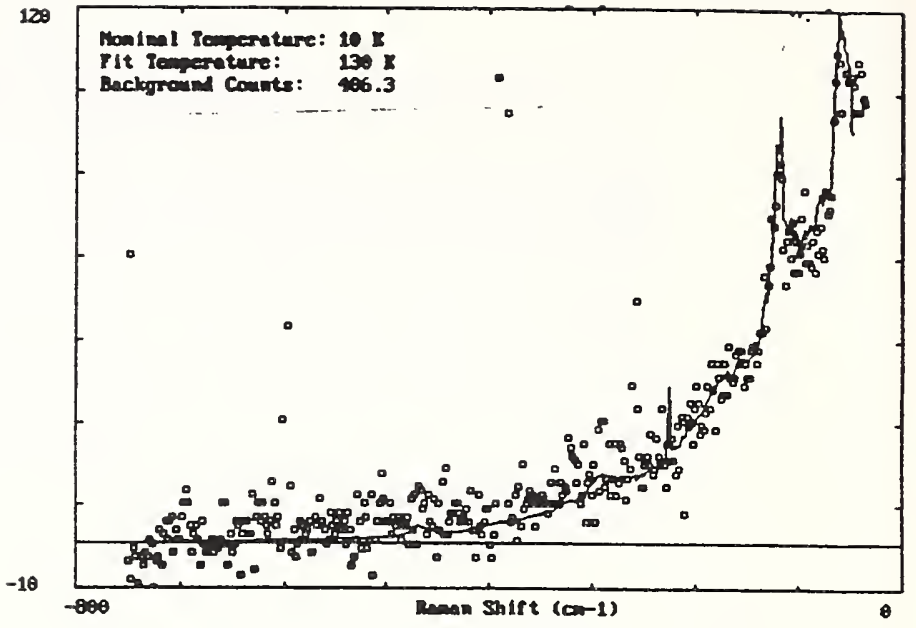

FIG. 3. A Stokes spectrum collected at a nominal temperature of $10 \mathrm{~K}$ is scaled by Eq. 1 and re-plotted along with the anti-Stokes spectrum. The fit to the data indicates a local temperature of $130 \mathrm{~K}$ within the laser spot, well above $T_{c}$ for this material.

\section{SUPERCONDUCTTVITY}

We now briefly introduce the topic of superconductivity. At temperatures below $\mathrm{T}_{c}$ in a superconductor, carrier-carrier attraction overcomes the coulomb repulsion, and the charged particles form pairs which move through the lattice without energy loss. This leads to the hallmark phenomena of zero resistivity and the Meissner effect of magnetic field expulsion and shielding. In Figure 4 these effects are illustrated for the case of Bi-based cuprates.

The pairing of carriers is restricted to those at low energies, leaving a gap in the density of states near the Fermi energy. One speaks of the value of this gap as the amount of energy required to break the pair and enter the region of finite density of states. The traditional measurement to determine this energy is electron tunneling where a dramatic change in the slope of an I vs. V curve allows a very high energy resolution of the gap value. Figure 5 illustrates this phenomenon for the $\mathrm{Bi}_{2} \mathrm{Sr}_{2} \mathrm{CaCu}_{2} \mathrm{O}_{8+\delta}$ superconductor.

While point contact tunneling offers very high energy resolution, it typically represents an average over a wide range of angles in $k$-space. This limits its utility in the spectroscopy of an anisotropic energy gap. In Figure 6 , we show 2 possible gap functions. The simplest is an $s$-wave gap with an isotropic $k$ dependence, as observed in many traditional low $\mathrm{T}_{c}$ materials. Conversely, an anisotropic $d$-wave gap has been suggested for the cuprates. This gap structure is identified with lobes (maxima) along the axes and nodes (minima $=$ zeros) at $45^{\circ}$ off the axes. Fundamentally, the Raman tensor can be sensitive to this anisotropy in a way that tunneling 

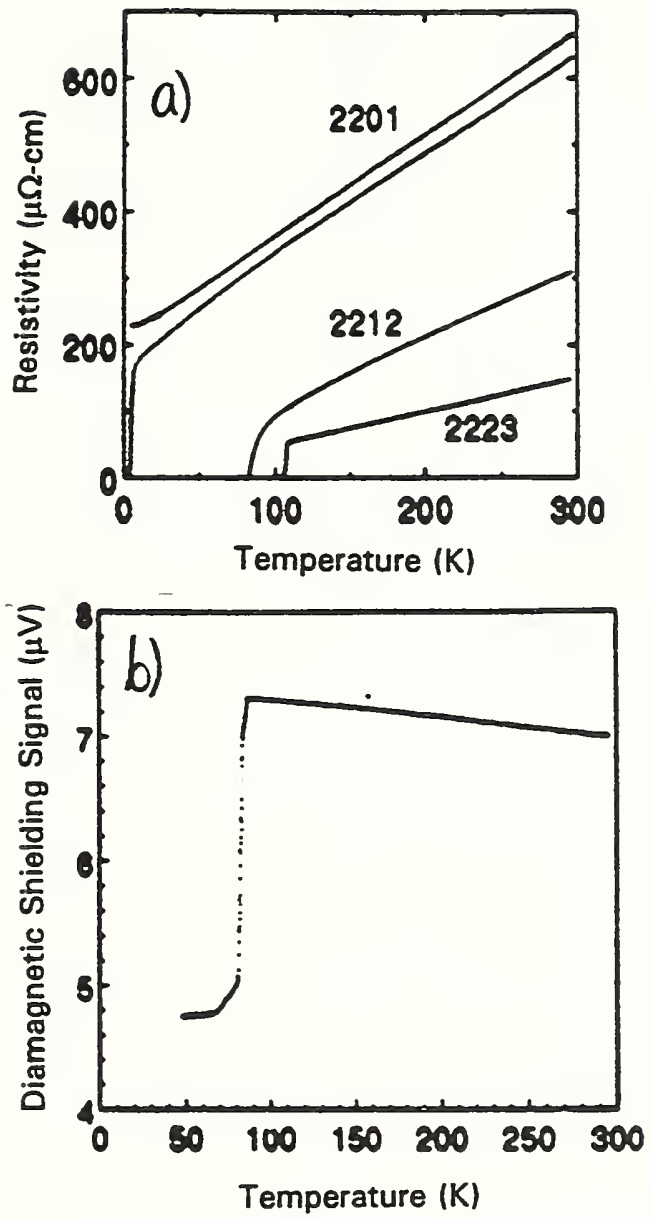

FIG. 4. a) Zero resistivity and b) diamagnetic shielding effects are shown for the Bi-based cuprates. Below $\mathrm{T}_{c}$, the resistance is identically zero and a magnetic field is screened from penetrating the bulk of the superconductor.

and many other techniques cannot.

Raman spectroscopy has been used to study superconductors since the days prior to the discovery of high $\mathrm{T}_{c}$ in the cuprates. Such studies investigate the behavior of the superconducting state through two related observable phenomena, the electronic continuum and the phonons. The electronic continuum reflects the underlying density of states and thus can provide information roughly analogous to tunneling experiments. Figure 7 gives an example from the work of M. V. Klein and S. B Dierker on the $17 \mathrm{~K}$ superconductor $\mathrm{V}_{3} \mathrm{Si}$. [2] Here the upper two spectra represent different symmetries (measured with different polarizations) below $T_{c}$. The lower 2 spectra show the behavior of the continuum in the same polarizations as above, but at a temperature above $T_{c}$.

Phonons interact with the continuum through a Fano interference, and thus their energy, width and intensity will change below $T_{c}$ in different ways, depending on whether they fall above or below the gap value. This phenomenon is demonstrated by the results of M. Kall et
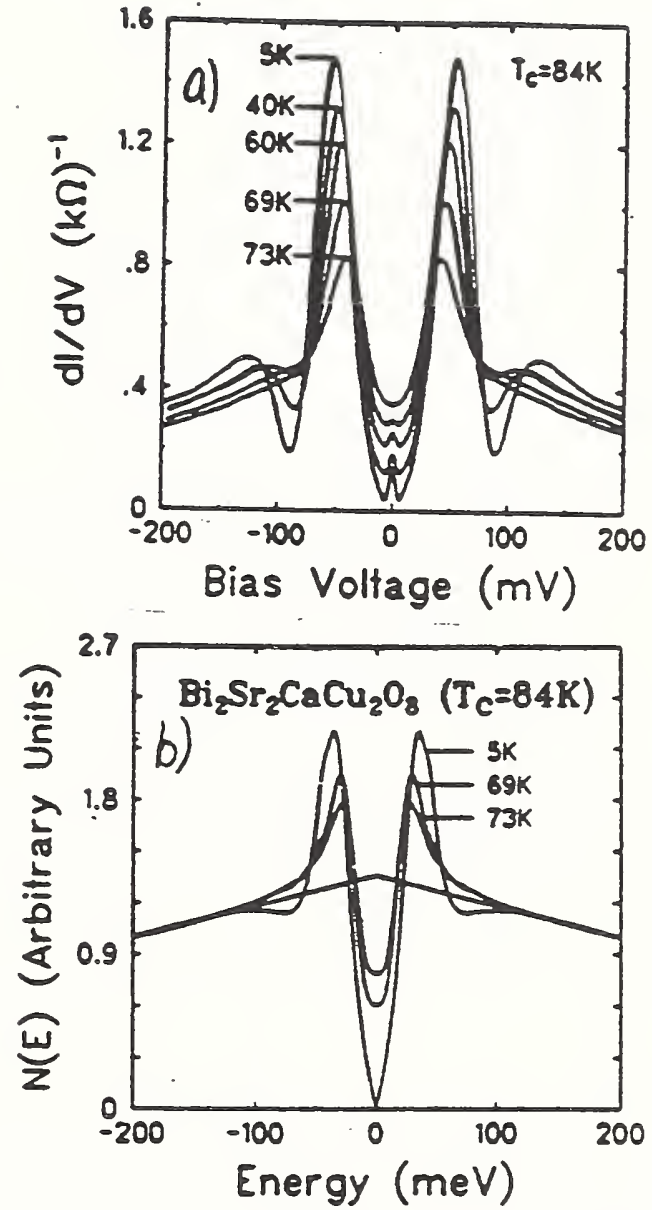

FIG. 5. (a)Electron tunneling data for the Bi2212 superconductor plotting the slope $(\mathrm{dI} / \mathrm{dV})$ of an I vs. V curve. This demonstrates the opening of a gap at low energies in the formation of a superconducting state. (b) A deconvolution of the tunneling data shows the effective density of states measured as a function of bias voltage.

al. as reproduced in Figure 8. [3] The $110 \mathrm{~cm}^{-1}$ phonon in $\mathrm{YBa}_{2} \mathrm{Cu}_{4} \mathrm{O}_{8}$ is measured as a function of temperature for samples with several different $T_{c}$. The phonon frequency, half-width and asymmetry all exhibit anomalies at $\mathrm{T}_{c}$.

\section{THEORY}

Through an appropriate choice of incident and scattered polarization vectors, it is possible to isolate the behavior of certain symmetries within a given crystal using Raman spectroscopy. This sensitivity applies to scattering both from phonons and electrons. Our aim is to exploit this capability to study possible anisotropy of the superconducting energy gap within the $\mathrm{CuO}_{2}$ planes. To explain how this is done, we must first discuss polarization selection rules and then elaborate on the common understanding of electronic Raman scattering. 

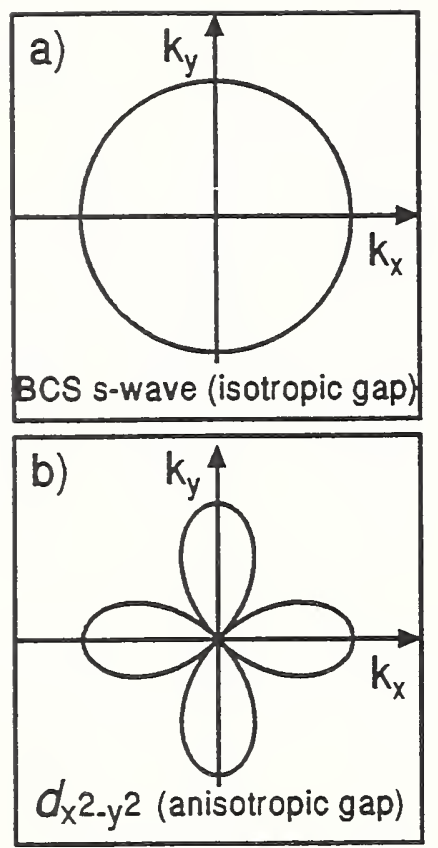

FIG. 6. 2 possible gap functions. a) an s-wave gap with an isotropic kdependence, and b) an anisotropic $d$-wave gap.

Group theoretical analysis is used, considering the cuprates within the $\mathrm{D}_{4 h}$ point group, to determine the polarization selection rules for excitations of different symmetries. Generally, the polarizations of incident and scattered radiation are indexed according to the $\mathrm{Cu}-\mathrm{O}$ bond directions, with $\mathrm{X}$ and $\mathrm{Y}$ taken parallel to the bonds. In this context, in-plane Raman scattering (measured with the incident and scattered light propagating along the $\mathrm{Z}$ direction parallel to the crystalline c-axis) measured in the $\mathrm{Z}(\mathrm{XY}) \bar{Z}$ polarization samples the $\mathrm{B}_{2 g}$ component, while $\mathrm{Z}(\mathrm{XX}) \bar{Z}$ samples a combination of the $\mathrm{A}_{1 g}$ and $\mathrm{B}_{1 g}$ symmetries. If $\mathrm{X}^{\prime}$ and $\mathrm{Y}^{\prime}$ denote axes rotated by $+45^{\circ}$ from $X$ and $Y$, respectively, then the $\mathrm{Z}\left(\mathrm{X}^{\prime} \mathrm{Y}^{\prime}\right) \bar{Z}$ polarization couples to excitations of $\mathrm{B}_{1 g}$ symmetry. For the unique case of $\mathrm{Bi}_{2} \mathrm{Sr}_{2} \mathrm{CaCu}_{2} \mathrm{O}_{8+\delta}, \mathrm{X}$ and $\mathrm{Y}$ are indexed along the $\mathrm{Bi}-\mathrm{O}$ bonds, which requires that the above designations be rotated by $45^{\circ}$ for comparison with other cuprates. In this cuprate, $X Y$ polarization samples $\mathrm{B}_{1 g}$ symmetry, $\mathrm{X}^{\prime} \mathrm{Y}^{\prime}$ samples $\mathrm{B}_{2 g}$, and $\mathrm{XX}$ couples to a combination of $\mathrm{A}_{1 g}$ and $\mathrm{B}_{1 g}$.

Recent theoretical work has shown that the scattering from different symmetries can reveal information about gap anisotropy due to differences in the portion of the Fermi surface (FS) sampled. [4] Although Raman scattering measures an average over the entire FS, certain angles are weighted more heavily in the integration. To see how this comes about we note that in high- $\mathrm{T}_{c}$ cuprates, intra-band electronic Raman scattering is dominated by

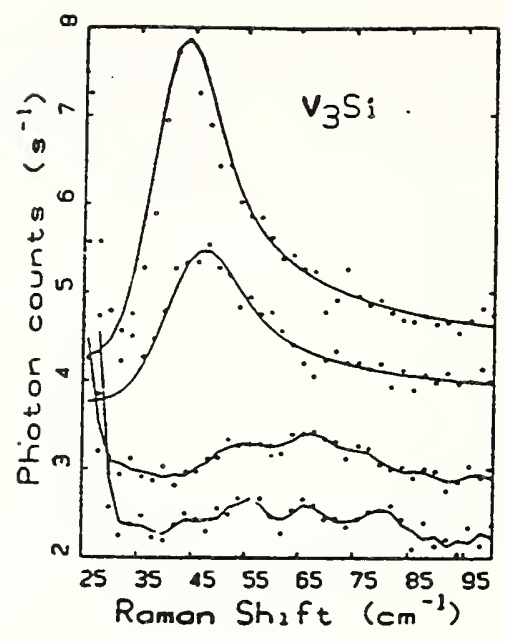

FIG. 7. Raman scattering data from the work of M. V. Klein and S. B Dierker on the $17 \mathrm{~K}$ superconductor $\mathrm{V}_{3} \mathrm{Si}$. Here the upper two spectra represent different symmetries (measured with different polarizations) below $\mathrm{T}_{c}$. The lower 2 spectra show the behavior of the continuum in the same polarizations as above, but at a temperature above $T_{c}$.

fluctuations in the effective mass around the FS given by the equation $\epsilon(\mathrm{k})=0$. In the appropriate limit where the wavevector transfer $\mathbf{q} \rightarrow 0$ we follow the calculations of Klein and Dierker [2] to arrive at an expression for the Raman scattering efficiency:

$\frac{d^{2} S}{d \omega d \Omega}=r_{o}^{2} \hbar N\left(E_{F}\right) \omega^{-1} m^{2}\left[\frac{<\mu^{-2} \lambda>-<\mu^{-1} \lambda>^{2}}{<\lambda>}\right]^{\prime \prime}$.

In this expression, $\omega$ is the frequency of Raman shift, $\Omega$ the solid angle scattered through, $r_{o}$ the Thomson radius of the electron, $N\left(E_{F}\right)$ the density of states at the Fermi level and $m$ the mass of the bare electron. The symbols $<>$ indicate that the quantitiy within is integrated around the entire FS. $\lambda$ is a complex function, the imaginary part of which is given by:

$$
\lambda=\frac{4|\Delta(\mathbf{k})|^{2}}{\sqrt{\omega^{2}-4|\Delta(\mathbf{k})|^{2}}} .
$$

with the restriction that $\omega^{2}>4 \Delta^{2}(\mathbf{k})$. There is no contribution from frequencies below the gap for a given wavevector k. In Eq. 1, $\mu$ is the Raman vertex, which is proportional to the fluctuation of the effective mass and is given in the non-resonant limit by:

$$
\mu_{i j}(\mathbf{k})=\frac{m}{\hbar^{2}} e^{I} \cdot \frac{1}{m^{*}} \cdot e^{S}=\frac{m}{\hbar^{2}} \sum_{i, j} e_{i}^{I} \cdot \frac{\partial^{2} \epsilon(k)}{\partial k_{i} \partial k_{j}} \cdot e_{j}^{S} .
$$

The angular dependencies of several relevant parameters in the above formula are displayed graphically in Figure 9. Figure 9(a) depicts a simplified Fermi surface, 

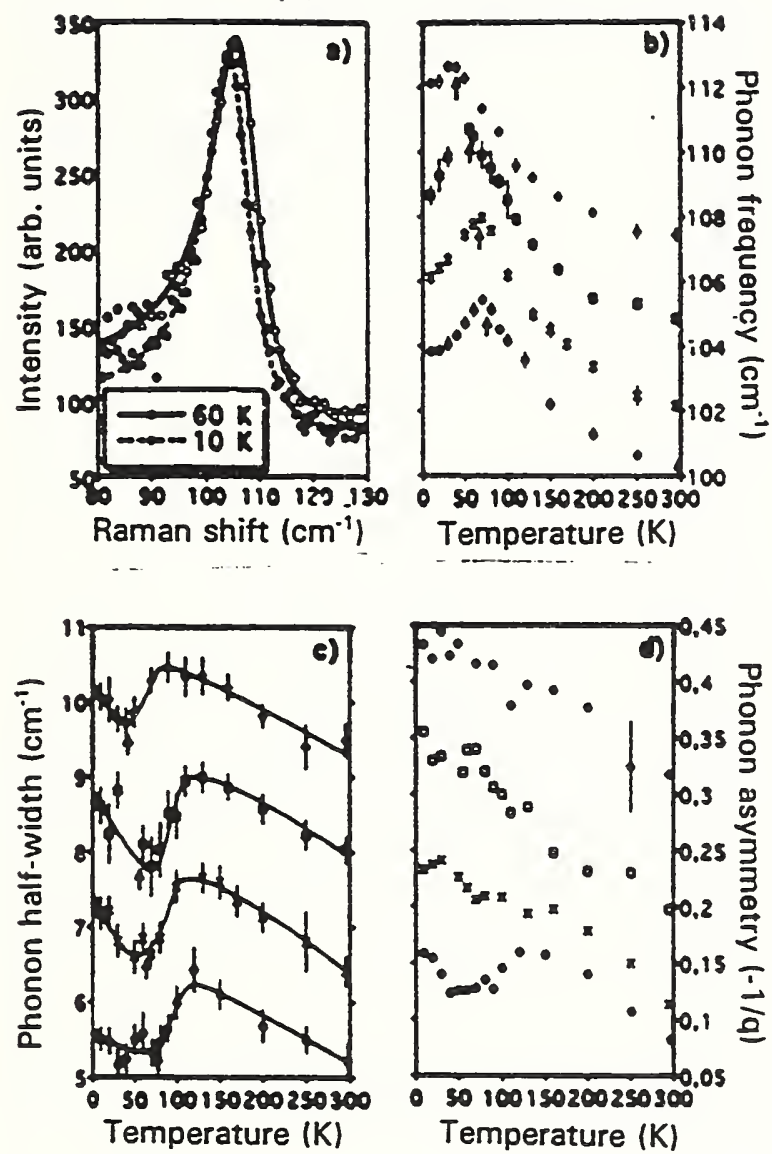

FIG. 8. The $110 \mathrm{~cm}^{-1}$ phonon in $\mathrm{YBa}_{2} \mathrm{Cu}_{4} \mathrm{O}_{8}$ is measured as a function of temperature for samples with several different $\mathrm{T}_{c}$. From the work of Kall et al. The phonon frequency, half-width and asymmetry all exhibit anomalies at $T_{c}$.

similar to that seen in angle resolved photoemission on $\mathrm{Bi}_{2} \mathrm{Sr}_{2} \mathrm{CaCu}_{2} \mathrm{O}_{8+\delta}$. This FS has been generated using a tight binding formulation with second nearest neighbor interaction such that

$\epsilon(k)=2 t\left[\cos \left(k_{x} a\right)+\cos \left(k_{y} a\right)\right]-4 s\left[\cos \left(k_{x} a\right) \cos \left(k_{y} a\right)\right]-E_{0}$.

with $\mathrm{t}=-1, \mathrm{~s}=-0.3$, and $\mathrm{E}_{0}=-1.16$. The angular dependence (including the sign) of the $A_{1 g}$ symmetry is displayed in the polar plot of Fig 9(b). Similarly, Fig. 9(c) and $9(\mathrm{~d})$ show the simpler angular weighting functions for the $B_{1 g}$ and $B_{2 g}$ symmetries, respectively. The magnitudes of the different components depend somewhat on the details of the FS chosen, but the shapes and angular dependencies are less sensitive.

To calculate the Raman scattering intensity for a given value of $\omega$, the expression in Eq. 2 is integrated around the entire FS with the appropriate weight function $(\mu)$ for each symmetry and the assumed gap $\Delta(\mathbf{k})$. It is important to note that the second term in Eq. 2, which
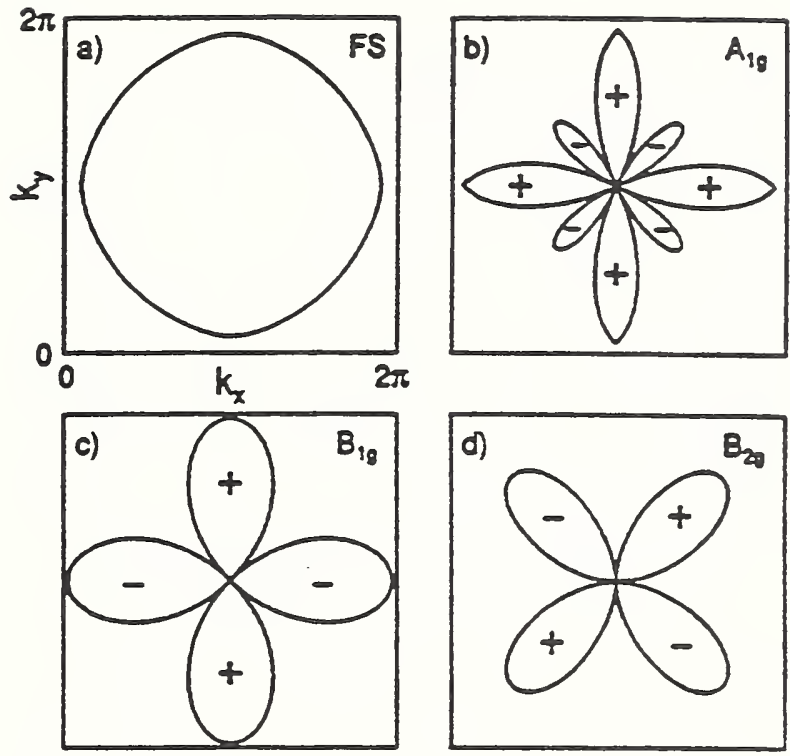

FIG. 9. a) A simplified Fermi surface, similar to that seen in angle resolved photoemission on $\mathrm{Bi}_{2} \mathrm{Sr}_{2} \mathrm{CaCu}_{2} \mathrm{O}_{8-\delta}$. b) The angular dependence (including the sign) of the $A_{13}$ symmetry is displayed in a polar plot. The simpier angular meighting functions for $c$ ) the $\mathrm{B}_{1 g}$ and d) $\mathrm{B}_{2,3}$ symmetries are also shown in polar plots.

takes into account the coulomb screening, is identically zero for all $\mathrm{B}_{1 g}$ and $\mathrm{B}_{2 g}$ since the equal areas of positive and negative sign cancel out. In $A_{1 g}$, however, this is not the case and the screening prevents the spectra in this symmetry from appearing as a linear combination of $\mathrm{B}_{1 g}$ and $\mathrm{B}_{2 g}$-like components, as it may appear from Figure 9(b).

\section{SAMIPLES}

At this point, we discuss the particular crystals studied with Raman scattering. The $\mathrm{Bi}_{2} \mathrm{Sr}_{2} \mathrm{CaCu}_{2} \mathrm{O}_{8-\varepsilon}$ (Bi2212) single crystals used in our experiments *ere gromn using a self-flux technique described in detail elsewhere. [5.6] The crystals were subsequently post-annealed in both high and low oxygen pressures to adjust the intercalated oxygen content and vary the hole-type carrier concentration. $[6,7]$ When annealed in air atmosphere, the crystals had a $\mathrm{T}_{c}$ of $83 \mathrm{~K}$. When annealed at $500^{\circ} \mathrm{C}$ in the highest oxygen pressure available. $2 \mathrm{kBar}$. the $T_{c}$ of the cry'stals was reduced to $5 \pi \mathrm{K}$. Such overdoped samples shor $50 \%$ lower resistivity. $\rho$, at room temperature and a significant $\mathrm{T}^{2}$ component in the temperature dependence. $\rho(\mathrm{T}) .[6,7]$ The entire underdoped portion of the phase diagram is accessible with anneals in vacuum. Figure 10 plots the temperature dependence of the resistivity: for Bi2212 samples post annealed in a variety of oxg.gen partial pressures. 

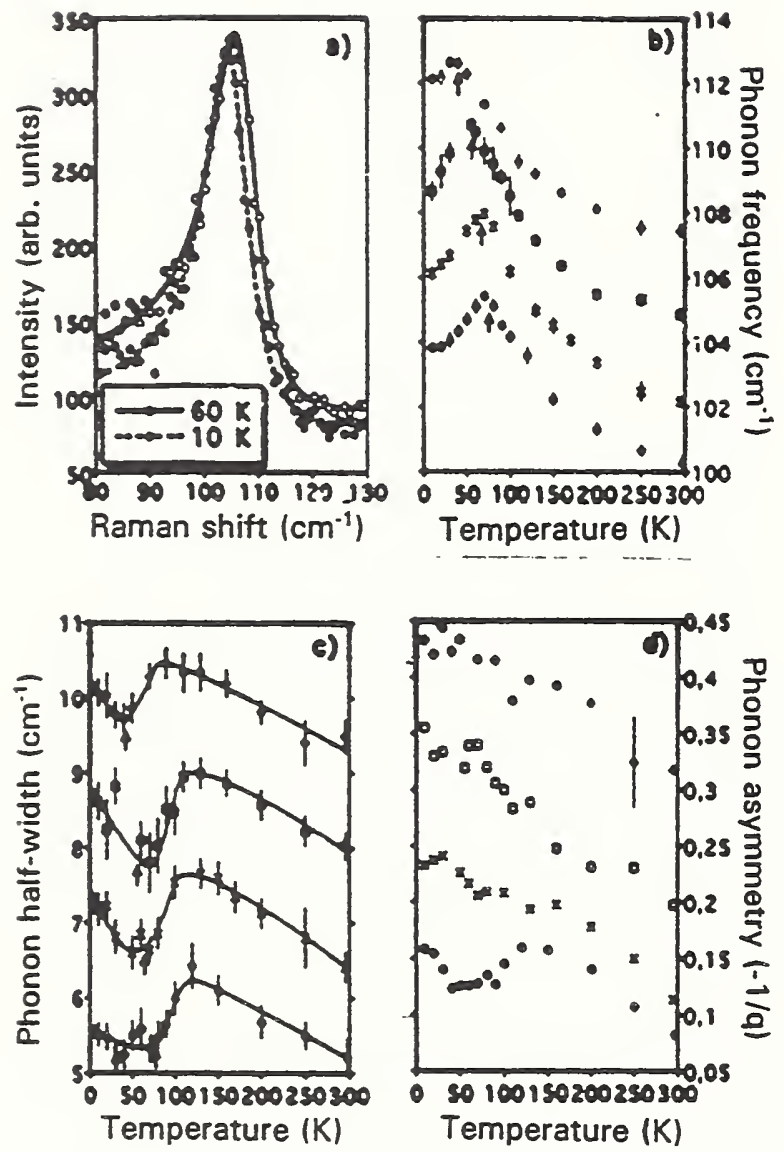

FIG. 8. The $110 \mathrm{~cm}^{-1}$ phonon in $\mathrm{YBa}_{2} \mathrm{Cu}_{4} \mathrm{O}_{8}$ is measured as a function of temperature for samples with several different $\mathrm{T}_{c}$. From the work of Kall et al. The phonon frequency, half-width and asymmetry all exhibit anomalies at $\mathrm{T}_{c}$.

similar to that seen in angle resolved photoemission on $\mathrm{Bi}_{2} \mathrm{Sr}_{2} \mathrm{CaCu}_{2} \mathrm{O}_{8+\delta}$. This FS has been generated using a tight binding formulation with second nearest neighbor interaction such that

$\epsilon(k)=2 t\left[\cos \left(k_{x} a\right)+\cos \left(k_{y} a\right)\right]-4 s\left[\cos \left(k_{x} a\right) \cos \left(k_{y} a\right)\right]-E_{0}$.

with $\mathrm{t}=-1, \mathrm{~s}=-0.3$, and $\mathrm{E}_{0}=-1.16$. The angular dependence (including the sign) of the $\mathrm{A}_{1 g}$ symmetry is displayed in the polar plot of Fig 9(b). Similarly, Fig. 9(c) and 9 (d) show the simpler angular weighting functions for the $\mathrm{B}_{1 g}$ and $\mathrm{B}_{2 g}$ symmetries, respectively. The magnitudes of the different components depend somewhat on the details of the FS chosen, but the shapes and angular dependencies are less sensitive.

To calculate the Raman scattering intensity for a given value of $\omega$, the expression in Eq. 2 is integrated around the entire FS with the appropriate weight function $(\mu)$ for each symmetry and the assumed gap $\Delta(\mathrm{k})$. It is important to note that the second term in Eq. 2, which
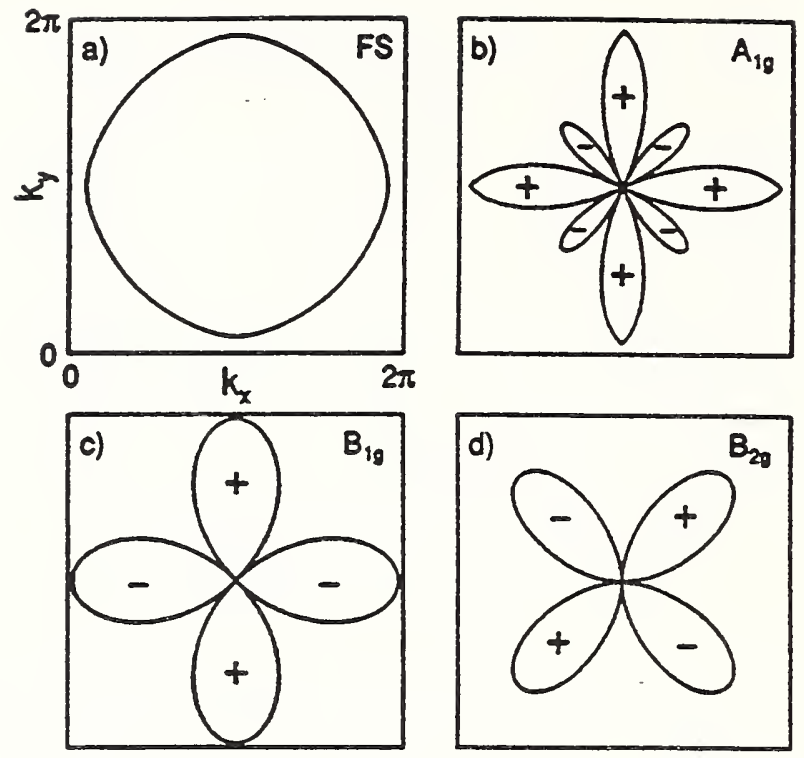

FIG. 9. a) A simplified Fermi surface, similar to that seen in angle resolved photoemission on $\mathrm{Bi}_{2} \mathrm{Sr}_{2} \mathrm{CaCu}_{2} \mathrm{O}_{8+\delta}$. b) The angular dependence (including the sign) of the $\mathrm{A}_{1 g}$ symmetry is displayed in a polar plot. The simpler angular weighting functions for $\mathrm{c}$ ) the $\mathrm{B}_{1 g}$ and d) $\mathrm{B}_{2 g}$ symmetries are also shown in polar plots.

takes into account the coulomb screening, is identically zero for all $\mathrm{B}_{1 g}$ and $\mathrm{B}_{2 g}$ since the equal areas of positive and negative sign cancel out. In $\mathrm{A}_{1 g}$, however, this is not the case and the screening prevents the spectra in this symmetry from appearing as a linear combination of $\mathrm{B}_{1 g}$ and $\mathrm{B}_{2 g}$-like components, as it may appear from Figure 9(b).

\section{SAMPLES}

At this point, we discuss the particular crystals studied with Raman scattering. The $\mathrm{Bi}_{2} \mathrm{Sr}_{2} \mathrm{CaCu}_{2} \mathrm{O}_{8+\delta}(\mathrm{Bi} 2212)$ single crystals used in our experiments were grown using a self-flux technique described in detail elsewhere. $[5,6]$ The crystals were subsequently post-annealed in both high and low oxygen pressures to adjust the intercalated oxygen content and vary the hole-type carrier concentration. $[6,7]$ When annealed in air atmosphere, the crystals had a $\mathrm{T}_{c}$ of $83 \mathrm{~K}$. When annealed at $500^{\circ} \mathrm{C}$ in the highest oxygen pressure available, $2 \mathrm{kBar}$, the $\mathrm{T}_{c}$ of the crystals was reduced to $57 \mathrm{~K}$. Such overdoped samples show $50 \%$ lower resistivity, $\rho$, at room temperature and a significant $T^{2}$ component in the temperature dependence, $\rho(T) .[6,7]$ The entire underdoped portion of the phase diagram is accessible with anneals in vacuum. Figure 10 plots the temperature dependence of the resistivity for Bi2212 samples post annealed in a variety of oxygen partial pressures. 


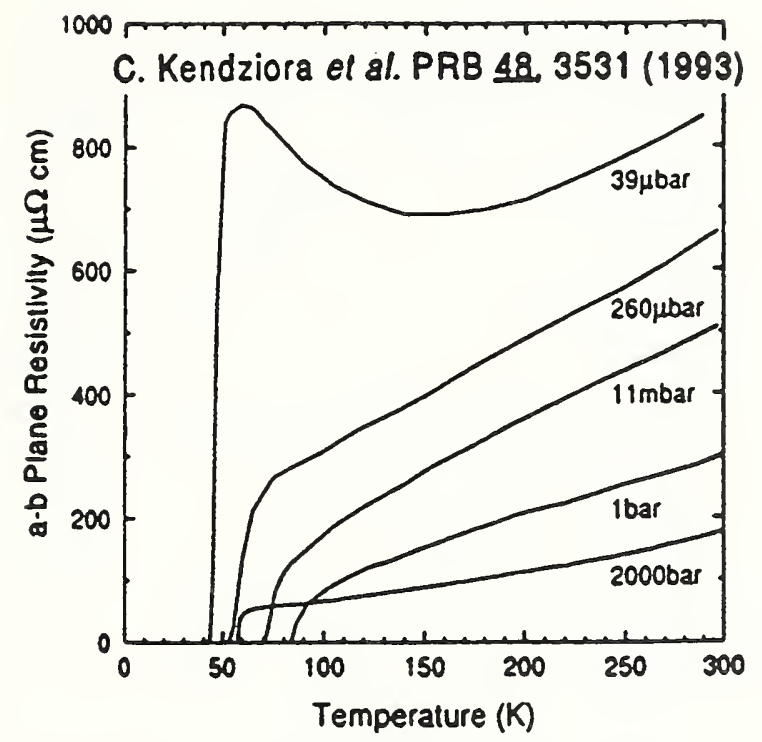

FIG. 10. The temperature dependence of the resistivity for Bi2212 samples post annealed in a variety of oxygen partial pressures.

Because it is not possible to directly measure the oxygen content of small single crystals, the $\delta$ parameter in these is determined by a measurement of $\mathrm{T}_{c}$, a knowledge of the annealing history, and a reference to the oxygen concentrations of much larger polycrystal samples. In Figure 11(a) we show the $\mathrm{T}_{c}$ dependence of $\delta$ reproduced from the work of R. Sieburger et al. [8] In Figure 11(b), we show the $\delta$ values for the samples studied with Raman scattering.

The $\mathrm{Tl}_{2} \mathrm{Ba}_{2} \mathrm{CuO}_{6+\delta}$ single crystals used in this study were provided by $\mathrm{T}$. A. Vanderah ${ }^{*}$ and were prepared by partially melting a stoichiometric mixture of $\mathrm{Tl}_{2} \mathrm{O}_{3}$, $\mathrm{BaO}_{2}$, and $\mathrm{CuO}$ in crimped gold tubing at $900^{\circ} \mathrm{C}$ in flowing oxygen. As grown, the superconducting transition temperature for these crystals is $78 \mathrm{~K}$, indicating that they are slightly overdoped relative to the optimal $\mathrm{T}_{c}$ of $90 \mathrm{~K}$ in this material. The broad transition width $\left(\Delta \mathrm{T}_{c}=5 \mathrm{~K}\right)$ indicates a slight oxygen inhomogeneity. When post-annealed in an oxygen atmosphere, the $T_{c}$ of these crystals decreases to $37 \mathrm{~K}$ and the transition sharpens $\left(\Delta \mathrm{T}_{c}=2 \mathrm{~K}\right)$. This process is reversible when the samples are re-annealed in vacuum. As measurements on polycrystalline samples of this material show, such reduced $\mathrm{T}_{c}$ phases eshibit the lower room temperature resistivity and $\rho(\mathrm{T}) \propto \mathrm{T}^{2}$ temperature dependence characteristic of overdoped cuprates. [9]

\section{RAMAN MEASUREMENTS}

Raman scattering measurements were made using the $514.5 \mathrm{~nm}$ line of an $\mathrm{Ar}^{+}$laser for excitation. The laser spot size, shape and power were adjusted according to
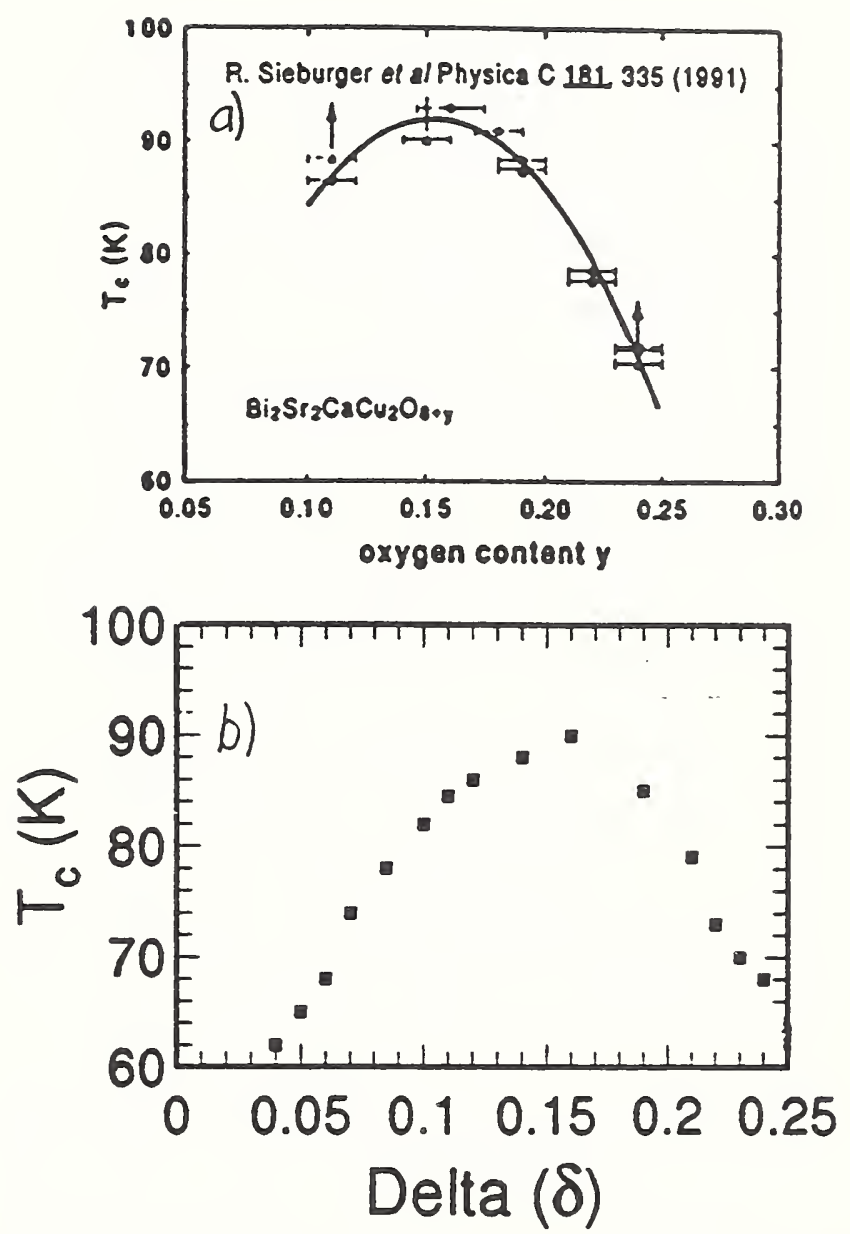

FIG. 11. (a) The $\mathrm{T}_{c}$ dependence of $\delta$ reproduced from the work of R. Seiburger et al (b) $\delta$ values for the samples studied with Raman scattering.

the sample size and degree of laser heating tolerable. The largest samples were studied using a line focus. Incident laser power densities ranged from $3-20 \mathrm{~W} / \mathrm{cm}^{2}$. The samples were cooled in a flowing $\mathrm{He}$ vapor to minimize laser heating, which we estimated to be $5-20 \mathrm{~K}$ above ambient by comparing the ratio of Stokes and Anti-Stokes spectra. The radiation collected in a back-scattering geometry was dispersed through a Dilor XY triple spectrometer in subtraction mode and detected with a nitrogen cooled CCD array. The Stokes spectra were measured for shifts from $30 \mathrm{~cm}^{-1}$ to $1100 \mathrm{~cm}^{-1}$ with a resolution of $9 \mathrm{~cm}^{-1}$.

In Figure 12 (a-c) we show the Raman spectra of Bi2212 crystals with a variety of oxygen contents measured with different incident and scattered light polarizations. [10] In Fig. 12(a) we show the data taken in $\mathrm{XX}$ polarization ( $\mathrm{A}_{1 g}$ symmetry); in Fig. 12(b), the XY polarization ( $\mathrm{B}_{1 g}$ symmetry) and in $12(\mathrm{c})$ the $\mathrm{X}^{\prime} \mathrm{Y}^{\prime}$ polarization ( $\mathrm{B}_{2 g}$ symmetry). For each sample, the data just above $T_{c}$ are overlayed with the spectrum measured well below $\mathrm{T}_{c}(30 \mathrm{~K})$. The data have been scaled by the thermal Bose factor, which "forces" the normal state spectra 

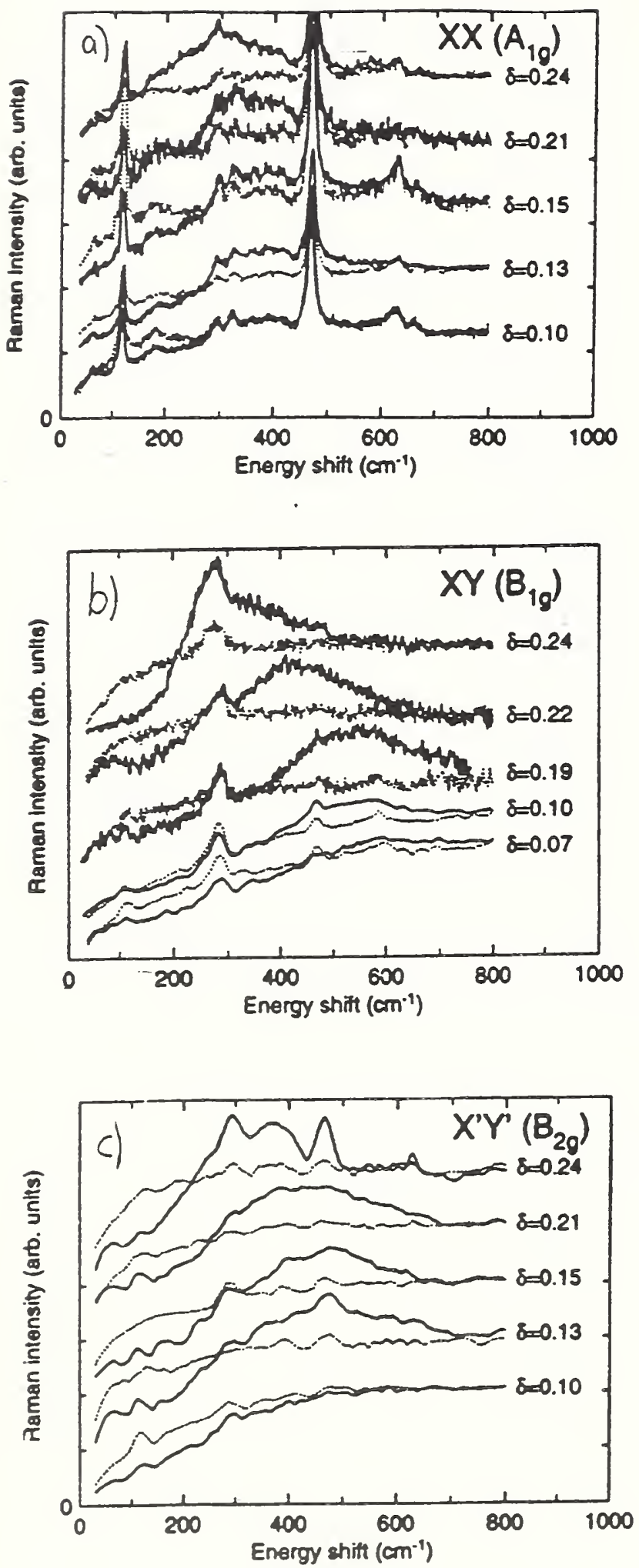

FIG. 12. Raman spectra of Bi2212 crystals with a variety of oxygen contents measured with different incident and scattered light polarizations. a) XX polarization ( $A_{1 g}$ symmetry); b) $\mathrm{XY}$ polarization $\left(\mathrm{B}_{1 g}\right.$ symmetry) and c) $\mathrm{X}^{\prime} \mathrm{Y}^{\prime}$ polarization $\left(\mathrm{B}_{2 \text { g }}\right.$ symmetry).

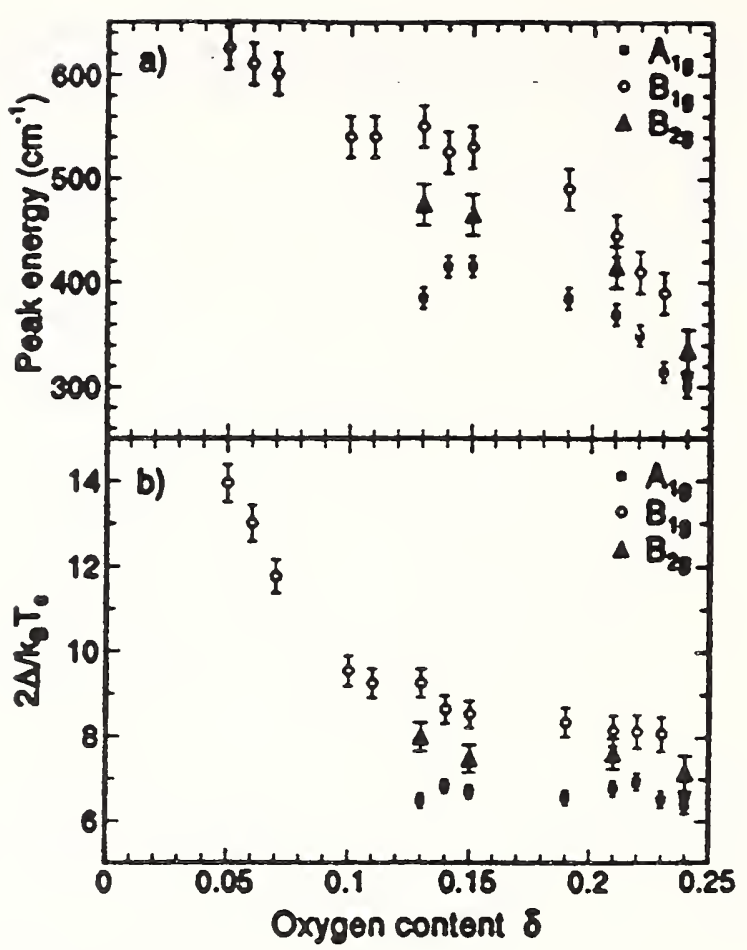

FIG. 13. A summary of the continuum peak energy data. from Fig. 12, as well as that from additional samples for which the spectra are not shown.

to extrapolate toward zero at low energy shifts, whereas the raw spectra are essentially "flat". A superconductivity related redistribution of the electronic continuum spectral weight is clearly observed in all overdoped samples. The peaks of these spectral features can be interpreted as the superconducting energy gap, in analogy to electron tunneling.

A summary of the continuum peak energy data from Fig. 12, as well as that from additional samples for which the spectra are not shown, is plotted in Figure 13. Several trends emerge when viewed in this way. As a function of doping, the superconductivity separates into three regimes, which we refer to as underdoped, optimal, and overdoped. Optimally doped samples, which correspond here to $\delta$ values of 0.13 to 0.24 , show distinctly different peak values in each of the three symmetries, indicative of an anisotropic energy gap. The continuum peak energies become less anisotropic as the doping level increases, until they coalesce for $\delta$ values greater than 0.25 . Thus, the overdoped regime can be considered to be characterized by an isotropic gap. Finally, in the underdoped regime, a continuum peak is observed only in $\mathrm{XY}$ polarization $\left(\mathrm{B}_{1 \mathrm{~g}}\right.$ symmetry). This unique situation may be related to a precipitous change in Fermi surface topology which takes place in underdoped samples as observed in recent angle resolved photoemission measurements. $[11,12\}$ As shown in Figure 13(b), the $A_{1 g}$ peak roughly scales with $\mathrm{T}_{c}$ throughout the optimally doped region, while the $\mathrm{B}_{1 g}$ 


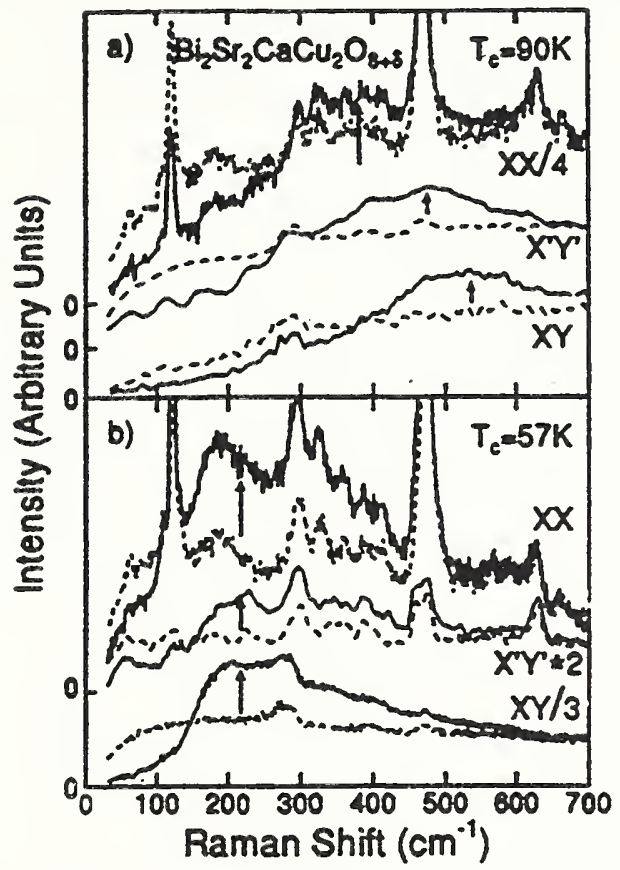

FIG. 14. The Raman scattering spectra in all three polarizations for (a) an optimally doped $\left(\mathrm{T}_{c}=90 \mathrm{~K}\right)$ and (b) a highly overdoped $\left(\mathrm{T}_{c}=57 \mathrm{~K}\right)$ Bi2212 crystal.

peak continues to rise even in underdoped samples where $\mathrm{T}_{c}$ has decreased considerably. A similar effect has been seen in underdoped $\mathrm{YBa}_{2} \mathrm{Cu}_{3} \mathrm{O}_{7-\delta}$. [13]

To focus on the overdoped portion of the phase diagram, we plot in Figure 14 the Raman scattering spectra in all three polarizations for (a) an optimally doped $\left(\mathrm{T}_{c}=90 \mathrm{~K}\right)$ and $(\mathrm{b})$ a highly overdoped $\left(\mathrm{T}_{c}=57 \mathrm{~K}\right)$ Bi2212 crystal. [14] As illustrated by the arrows, the $\mathrm{T}_{c}=90 \mathrm{~K}$ sample clearly exhibits a symmetry dependence to the continuum peak energies, while the gap in the $\mathrm{T}_{c}=57 \mathrm{~K}$ crystal is symmetry independent. The continuum peak energies for the $\mathrm{T}_{c}=90 \mathrm{~K}$ sample are $8.5 \mathrm{k}_{B} \mathrm{~T}_{c}\left(\mathrm{~B}_{1 g}\right)$, $7.5 \mathrm{k}_{B} \mathrm{~T}_{c}\left(\mathrm{~B}_{2 g}\right)$ and $6.2 \mathrm{k}_{B} \mathrm{~T}_{c}\left(\mathrm{~A}_{1 g}\right)$, while for the $\mathrm{T}_{c}=57 \mathrm{~K}$ crystal, the peak energies fall at $5.3 \mathrm{k}_{B} \mathrm{~T}_{c}$ in all three symmetries. This illustrates two pronounced effects of overdoping: (1) the superconducting energy gap becomes symmetry independent, and (2) the size of the gap decreases even faster than $\mathrm{T}_{c}$ does.

Similar effects are observed in $\mathrm{Tl}_{2} \mathrm{Ba}_{2} \mathrm{CuO}_{6+\delta}$ crystals, where it is possible to go even further into the underdoped regime. In Figure 15, we plot the Raman spectra for two T12201 crystals at different oxygen contents, where (a) $\mathrm{T}_{c}=78 \mathrm{~K}$ and (b) $\mathrm{T}_{c}=37 \mathrm{~K}$. [14] For the $\mathrm{T}_{c}=78 \mathrm{~K}$ crystal, which is still in the optimally doped regime, the continuum peak energies (denoted by arrows) are $7.2 \mathrm{k}_{B} \mathrm{~T}_{c}\left(\mathrm{~B}_{1 g}\right)$ and $5.8 \mathrm{k}_{B} \mathrm{~T}_{c}\left(\mathrm{~A}_{1 g}\right)$. No Raman scattering signal was observed in $\mathrm{XY}$ polarization, corresponding to $\mathrm{B}_{2 g}$ symmetry. For the overdoped $\mathrm{T}_{c}=37 \mathrm{~K}$ sample, the continuum peak energies are $3.9 \mathrm{k}_{B} \mathrm{~T}_{c}$ in both symmetries, consistent with the observations from overdoped

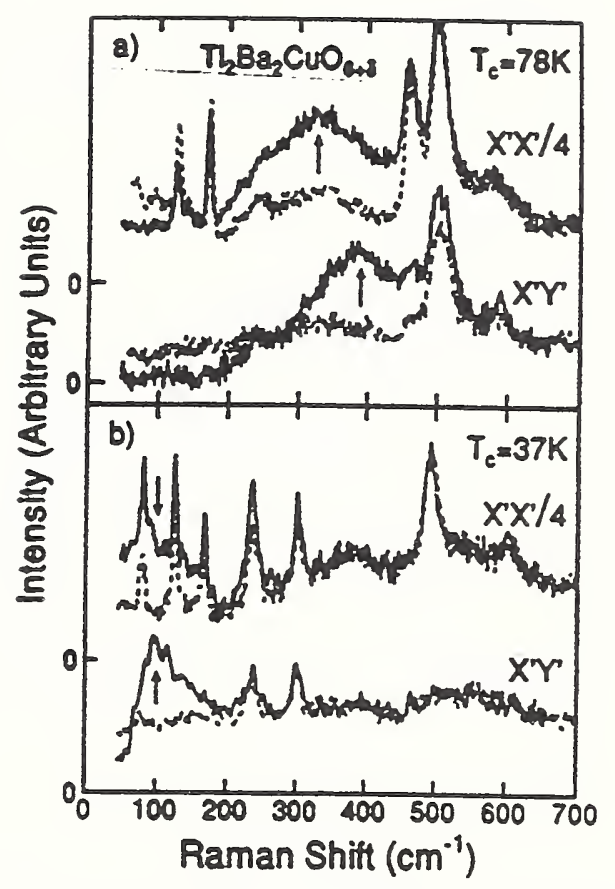

FIG. 15. the Raman spectra for two T12201 crystals at different oxygen contents, where (a) $\mathrm{T}_{c}=78 \mathrm{~K}$ and (b) $\mathrm{T}_{c}=37 \mathrm{~K}$.

Bi2212 crystals. The gap value of $3.9 \mathrm{k}_{B} \mathrm{~T}_{c}$ is extrordinarily low for the cuprates, and indicates that the strength of the pairing in these materials is highly sensitive to the carrier concentration.

In Figure 16, we plot the ratio $\mathrm{I}_{S} / \mathrm{I}_{N}(\mathrm{~S} \equiv$ superconducting, $\mathrm{N} \equiv$ normal) of the Raman scattering intensities for $\mathrm{Bi}_{2} \mathrm{Sr}_{2} \mathrm{CaCu}_{2} \mathrm{O}_{8+\delta}$ from Figure 14. The ratios have been scaled and offset so that XX and XY polarizations can be viewed simultaneously and compared with calculations based on the symmetry dependence of the Raman vertices. In Fig. 16(a), the $\mathrm{XX}\left(\mathrm{A}_{1 g}\right)$ and (smoothed) $\mathrm{XY}$ $\left(B_{1 g}\right)$ data for the $T_{c}=90 \mathrm{~K}$ sample are scaled by a factor $\Delta=265 \mathrm{~cm}^{-1}$ and compared with the calculations of Eq. 2 using a gap with $d$-wave symmetry. In Fig. 16(b), the $\mathrm{XX}$ and $\mathrm{XY}$ spectra for the $\mathrm{T}_{c}=57 \mathrm{~K}$ sample are scaled by $\Delta=110 \mathrm{~cm}^{-1}$ and overlaid with the calculation using an isotropic gap $\Delta(\mathrm{k})=1$.

Several factors affecting the calculated spectra should be noted. (1) We have employed a broadening of $0.3 \Delta$ for $T_{c}=90 \mathrm{~K}$ and $0.4 \Delta$ for $T_{c}=57 \mathrm{~K}$ to avoid singularities in the calculations, as well as to account phenomenologically for some transition width and the finite lifetime of the excited carriers. This gaussian distribution of gap sizes can account for non-zero intensity below $2 \Delta$ in the case of an isotropic gap, whereas lifetime broadening effects alone cannot. [15] The introduction of a similar convolution has been necessary to adequately fit observed tunneling data in the cuprates. (2) Due to the effects of screening, the calculated $A_{1 g}$ curve had to be scaled up 


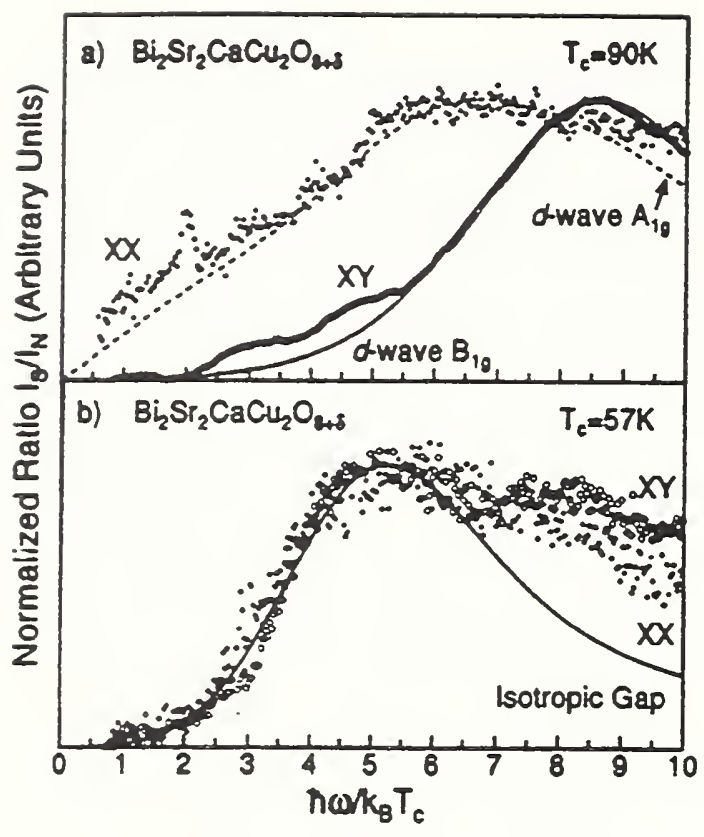

FIG. 16. The ratio $I_{S} / I_{N}$ (S $\equiv$ superconducting, $\mathrm{N} \equiv$ normal) of the Raman scattering intensities for $\mathrm{Bi}_{2} \mathrm{Sr}_{2} \mathrm{CaCu}_{2} \mathrm{O}_{8+\delta}$ from Figure 14.

to the same peak strength as that in $\mathrm{B}_{1 g}$, even though the observed XX scattering intensity is stronger than in $X Y$. (3) This theory does not account for scattering in the normal state, and thus the calculations do not resemble the observed spectra for $\omega>2 \Delta$. The quality of the fits in this frequency range can be greatly improved by inserting a normal state self-energy component physically related to electron-electron or spin-fluctuation scattering. [16]

A comparison of the optimally doped spectra with the $d$-wave calculations show some remarkable similarities. The model successfully predicts an $\omega^{3}$ shape in the $\mathrm{B}_{19}$ symmetry, as well as the nearly linear extrapolation to zero in $A_{1 g}$. Somewhat better fits to the peak frequency of the $A_{1 g}$ ratio have been made using data from different samples as well as a modified Fermi surface. Consideration of a less anisotropic gap without nodes moves the predicted $\mathrm{A}_{1 g}$ peak closer to $2 \Delta$ but suggests that (except for the smearing) the low frequency scattering in each symmetry should go to zero at the gap minimum. Therefore, the data on optimally doped crystals demonstrate that the gap: (1) is anisotropic, (2) has maxima along $\mathrm{k}_{x}=0$ and $\mathrm{k}_{y}=0$, and (3) has minima $\ll 2 \Delta$.

Devereaux has has recently proposed that impurity scattering can distinguish whether the anisotropic gap has nodes required by symmetry. [16] Excess oxygen is clearly not the ideal impurity for the cuprates because its dominant effect is to raise carrier concentration. Nonetheless, the prediction that the peak frequencies should retain their symmetry dependent anisotropy for a $d$-wave but coalesce in the $s$-wave case bears on the observation of the data in Fig. 16(b). Here the ratios of XX and XY spectra are both well fit by a broadened isotropic gap. The Tl 2201 data in Fig. 15 display similar behavior, but there the spectra are experimentally limited in the region of interest $(\omega<\Delta)$.

It is crucial to note that the gap does not scale with $\mathrm{T}_{c}$ for any symmetry, but rather is monotonically decreasing with doping level to $2 \Delta / \mathrm{k}_{B} \mathrm{~T}_{c}=3.9(5.5)$ in samples of $\mathrm{Tl} 2201$ ( $\mathrm{Bi} 2212$ ) with $\mathrm{T}_{c}=37 \mathrm{~K}(57 \mathrm{~K})$. Indeed, the isotropic gap seen in Raman scattering can be considered characteristic of heavily overdoped cuprates. Recently, a nearly isotropic gap $\left(2 \Delta / \mathrm{k}_{B} \mathrm{~T}_{c} \approx 4.1-4.9\right)$ has been seen in $\mathrm{Nd}_{1.85} \mathrm{Ce}_{0.15} \mathrm{CuO}_{4}$. [17] Because at this cerium concentration the resistivity of this material is typical of the overdoped cuprates $-\rho(T) \propto T^{2}-$ we speculate that each cuprate system will manifest an isotropic gap at high doping levels. The greatly reduced value of this gap: even when the reduction in critical temperature is taken into account, is further evidence that at sufficiently high doping levels the cuprates manifest conventional weakcoupling superconductivity.

\section{CONCLUSIONS}

We have performed electronic Raman scattering measurements on $\mathrm{Bi}_{2} \mathrm{Sr}_{2} \mathrm{CaCu}_{2} \mathrm{O}_{8+\delta}$ and $\mathrm{Tl}_{2} \mathrm{Ba}_{2} \mathrm{CuO}_{6+\delta}$ high temperature superconductors with variations in the oxygen content. We compare the observed spectra in the superconducting state with that calculated using order parameters of isotropic $s$ and $d$-wave symmetries. Near optimal doping, both materials show a very strong-coupling gap $\left(2 \Delta / \mathrm{k}_{B} \mathrm{~T}_{c} \sim 8\right)$ with anisotropic features suggesting nodes. However, we cannot distinguish whether the order parameter changes sign around the Fermi surface. When the carrier concentration is raised. both materials exhibit an isotropic gap at much lower energies, consistent with a more conventional s-wave order parameter at values approaching the weak-coupling limit $\left(2 \Delta / \mathrm{k}_{B} \mathrm{~T}_{c} \sim 4\right)$. In the underdoped regime, a continuum peak is observed only in $\mathrm{B}_{1 g}$ symmetry, at a value which increases even as $\mathrm{T}_{c}$ decreases.

* Ceramics Division, National Institute of Standards and Technology.

[1] J. G. Bednorz and K. A. Muller, Z. Phys. B64, 189; (1986).

[2] M. V. Klein and S. B. Dierker, Phys. Rev. B29, 4976 (1984).

[3] M. Kall et al. Phys. Rev. B53, 3566 (1996).

[4] T. P. Devereaux et al., Phys. Rev. Lett. 72, 396 (1994). 
[5] C. Kendziora, L. Forro, D. Mandrus, J. Hartge, P. Stephens, L. Mihaly, R. Reeder, D. Moecher, M. Rivers and S. Sutton, Phys. Rev. B45, 13025 (1992).

[6] C. Kendziora, R. J. Kelley, E. Skelton and M. Onellion, Physica C 257, 74 (1996).

[7] C. Kendziora, Michael C. Martin, J. Hartge, L. Mihaly and L. Forro, Phys. Rev B48, 3531 (1993).

[8] R. Sieburger et al, Physica C 181, 335 (1991).

[9] Y. Kubo, Y. Shimakawa, T. Manako, and H. Igarashi, Phys. Rev. B43, 7875 (1991).

[10] C. Kendziora and A. Rosenberg, Phys. Rev. B52, R9867 (1995).

[11] D. S. Marshall et al., Phys. Rev. Lett.76, 4841 (1996).

[12] S. LaRosa, I. Vobornik, H. Berger, G. Margaritondo, C. Kendziora, R. J. Kelley, Jian Ma, and M. Onellion, unpublished.

[13] F. Slakey, M. V. Klein, J. P. Rice, D. M. Ginzberg, Phys. Rev. B42, 2643 (1990).

[14] C. Kendziora, R. J. Kelley and M. Onellion, Phys. Rev. Lett. 77, 727 (1996).

[15] S. L. Cooper and M. V. Klein, Comm. Cond. Mat., 15, 99 (1990).

[16] T. P. Devereaux, Phys. Rev. Lett. 74, 4313 (1995).

[17] B. Stadlober, G. Krug, R. Nemetschek, R. Hackl, J. L. Cobb and J. T. Markert, Phys. Rev. Lett. 74, 4911 (1995).

\section{Discussion}

Q I have a very naive question. Do you know what is the physics that is involved in the overdoped regime?

A Well, the general modus operandi is that you go farther away from the anti ferro magnetic behavior and more into the realm of real metal behavior. 


\title{
Raman Spectroscopy of Ultrathin Organic Films and Ceramic Precursors
}

\author{
John F. Rabolt \\ Materials Science Department \\ University of Delaware \\ Newark, $D E$
}

\begin{abstract}
This paper presents a number of examples of Raman spectroscopy conducted in an industrial research laboratory. The topics include the use of integrated optics to enhance Raman scattering from thin films; the effect of orientation in anisotropic films; tailoring of the optical fields intensity in AB block copolymers; the use of Fourier transform Raman spectroscopy with fiber optics collection system to obtain Raman spectra of polymer films; and the determination of the backbone conformation in ceramic precursor polymers.
\end{abstract}

\section{Introduction}

The technique of Raman scattering spectroscopy finds a lot of applications in the industrial environment. In this paper we present several highlights to illustrate this technique using the scattering by thin films as the means of our demonstration. The description of integrated optics to enhance Raman scattering is followed by four parts which deal with special aspects of the physics and chemistry of thin films as studied by Raman spectroscopy.

\section{Use of Integrated Optics}

For many years it has been known in the integrated optics community that if you have a material with a high index of refraction bounded by media with a lower index of refraction, a light beam coupled into the film by a prism or grating under a high angle of incidence can undergo total internal reflection (see Fig. 1). The beam suffers phase shifts $\phi_{12}$ and $\phi_{32}$ upon reflection, and a phase shift $\delta$ upon passage through the film. The solution to the Maxwell equations subject to appropriate boundary conditions yields an eigenvalue equation which must be satisfied by these phase shifts,

$$
\phi 12+\phi 32+2 \delta=2 m \pi,
$$

where

$$
\mathrm{m}=0,1,2,3, . .
$$

and with $d$ given by

$$
\mathrm{d}=\left(2 \pi \mathrm{n}_{2} \mathrm{~d} / \lambda_{0}\right) \cos \theta_{2},
$$

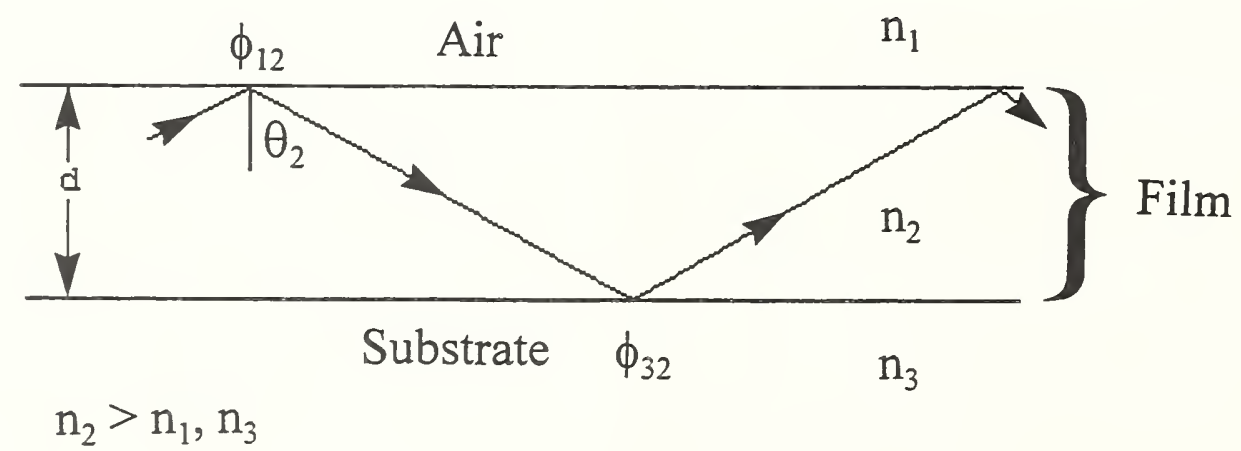

Fig. 1. Optics of total internal reflection in a thin film. 
where $d$ is the thickness of the film and $\lambda_{0}$ is the wavelength of the light beam in vacuum. The eigenfunctions in turn are the optical field intensities inside the film as well as the bounding media [1-4]. A plot of these is shown in Fig. 2 for the first three modes $(m=1,2,3)$. For higher order modes the field penetrates more and more into the substrate because there is closer matching between the index of the film and that of the substrate than of the air.

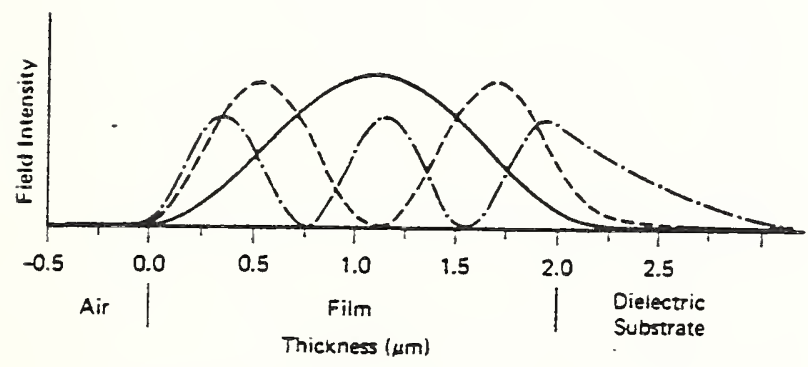

Fig. 2. Optical field intensity in a thin film bounded by air and a dielectric substrate [1].

If the film is "thin", it will act as a wave guide. The advantages of wave guide Raman spectroscopy are 1) there is a high optical field in a thin layer, 2) Raman scattering is produced by many molecules, 3) the guiding streak of the exciting beam of light can be focused on the slit of a spectrometer, and 4) the optical configuration can be easily adapted to a standard spectrometer. Table I lists for the first three modes the angle of internal reflection and the number of reflections per centimeter, for each state of polarization, transverse electric (TE) and transverse magnetic (TM), respectively, of the optical field [2]. The values are calculated for a $1 \mathrm{~mm}$ thick film of polystyrene $(n=1.6008)$ on a pyrex glass substrate $(n=1.4780)$ for a wavelength $\lambda_{0}=488.0 \mathrm{~nm}$, where the TE mode has its electric vector parallel to the surface of the film (perpendicular to the plane of incidence); the TM mode has the electric vector in the plane of incidence. For $\mathrm{m}=0$ the TE mode experiences 650 reflections per centimeter, which compares to only 15 to 20 reflections $/ \mathrm{cm}$ in a standard attenuated total reflection (ATR) experiment in the infrared. For higher orders there is a correspondingly larger number of bounces which leads to a strongly enhanced
Table I. Advantages of Wave Guide Raman Spectroscopy.

\begin{tabular}{ccccc}
\hline Polarization & $\mathrm{m}$ & 0 & 1 & 2 \\
\hline \multirow{2}{*}{ TE } & $\theta$ & 82.6 & 74.2 & 68.0 \\
& $\mathrm{R} / \mathrm{l}^{*}$ & 650 & 1322 & 2015 \\
& & & & \\
$\mathrm{TM}$ & $\theta$ & 82.2 & 74.5 & 67.4 \\
& $\mathrm{R} / \mathrm{l}^{*}$ & 682 & 1366 & 2079 \\
\hline \hline
\end{tabular}

$* \mathrm{R} / \mathrm{l}=$ Number of reflections per centimeter.

interaction of the light with the medium of the film. In addition to this enhancement there is the fact that the narrow streak of the laser beam in the film and hence the line-like source of the Raman radiation can very efficiently be coupled to the entrance aperture of a slit spectrometer with little loss of light.

Another method of assessing the advantages of using the thin film technique is to compare the results with it with those obtained from a bulk sample. In order to do this properly one has to normalize the conditions with respect to resolution and the power of the incident light.

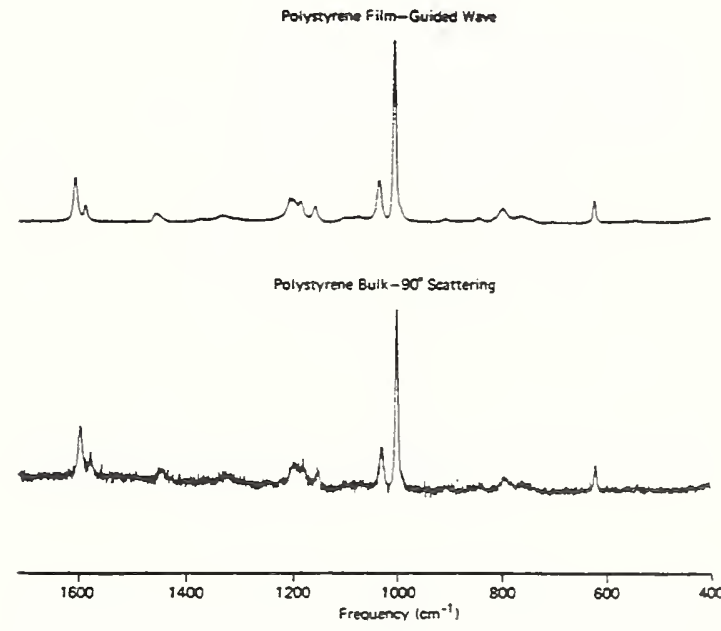

Fig. 3. Comparison of the Raman spectrum of a thin film of polystyrene with that of the bulk material.

Figure 3 shows such a comparison for the Raman spectrum of a polystyrene film of thickness $1.1 \mu \mathrm{m}$ and the corresponding spectrum of the bulk in the form of polystyrene powder [2]. Even though the experiments were not optimized, the comparison clearly demonstrates the advantages of the thin film method. 


\section{Orientation in Anisotropic Thin Films}

In the previous section the thin film acted not only as a wave guide but it was also the medium which generated the Raman scattered light which was then recorded (Fig. 3). It is also possible to have the wave guide film carry another (or more) thin film(s) which can be studied by Raman spectroscopy. Figure 4 gives a schematic diagram of the experimental arrangement. Also shown is the wave guide assembly composed of

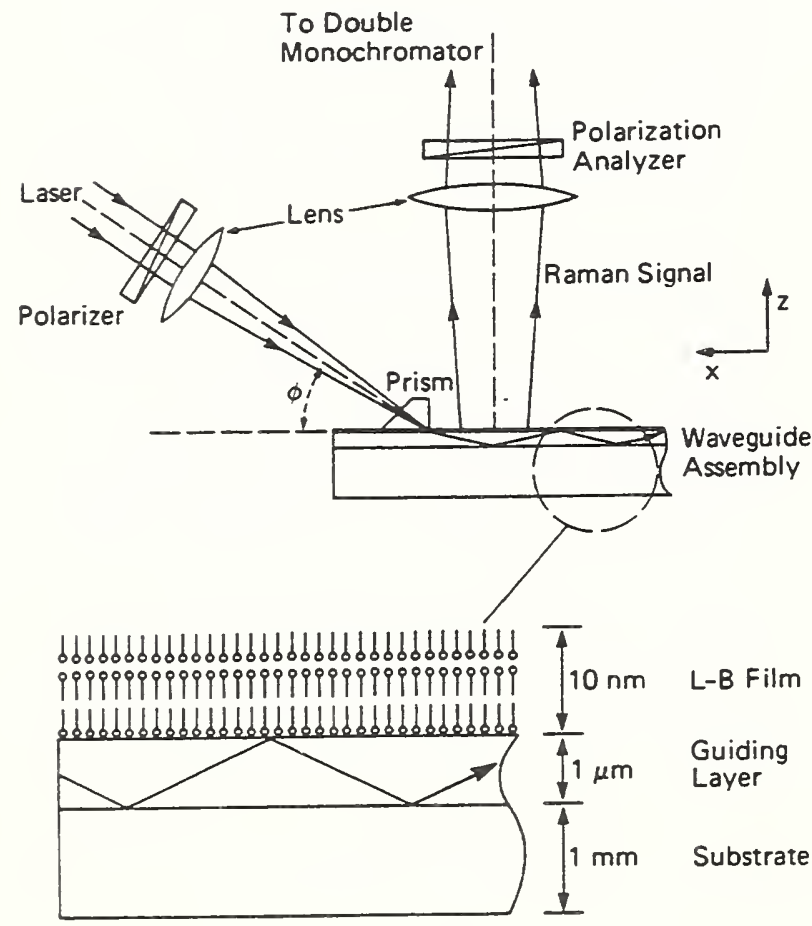

Fig. 4. Schematic diagram of wave guide Raman scattering and wave guide structure for studies of Langmuir-Blodgett films.

a $1 \mathrm{~mm}$ thick substrate, a $1 \mu \mathrm{m}$ thick guiding layer, on top of which is a Langmuir-Blodgett (LB) film of thickness $10 \mathrm{~nm}$. Depending on the indices of refraction of the LB and guiding layers, the evanescent electric field of the wave in the guiding layer penetrates into the LB film or the LB film itself may become part of the wave guide, giving rise to Raman scattering [5]. The evanescent field from the guiding layer is highly polarized, depending on whether the TE or TM mode has been selected. This polarized light can be used to explore the anisotropic character of LB films, determining information about both the orientation and lateral order of molecules.

In the laboratory frame of reference the incident laser light propagates along the $-X$ direction while the scattered light is viewed along the $Z$ direction. The incident light can be polarized either along the $Z$ or $Y$ direction, while the analyzer for the scattered light can be placed parallel to either the $\mathrm{X}$ or $\mathrm{Y}$ axis. Figure 5 shows the Raman spectrum in the region of the $\mathrm{C}-\mathrm{H}$ stretching vibration obtained in this manner for an LB multilayer of cadmium arachidate ( $\mathrm{CdA}$ ) consisting of four bi-layers of total thickness $20 \mathrm{~nm}$. The figure labels the optical geometry used, i.e., $X(Y Y) Z$ and $X(Y X) Z$. These are of the now standard form $A(B C) D$. With respect to a laboratory frame of coordinates, A and D designate the direction of propagation of the incident laser and the observed scattered light, respectively, while the labels $B$ and $C$ inside the parentheses give the directions of polarization of the incident and observed scattered radiations, respectively [6]. The observed polarization of the scattered radiation gives evidence about the orientation of the hydrocarbon chains of the CdA molecules (see Fig. 6) on the surface.

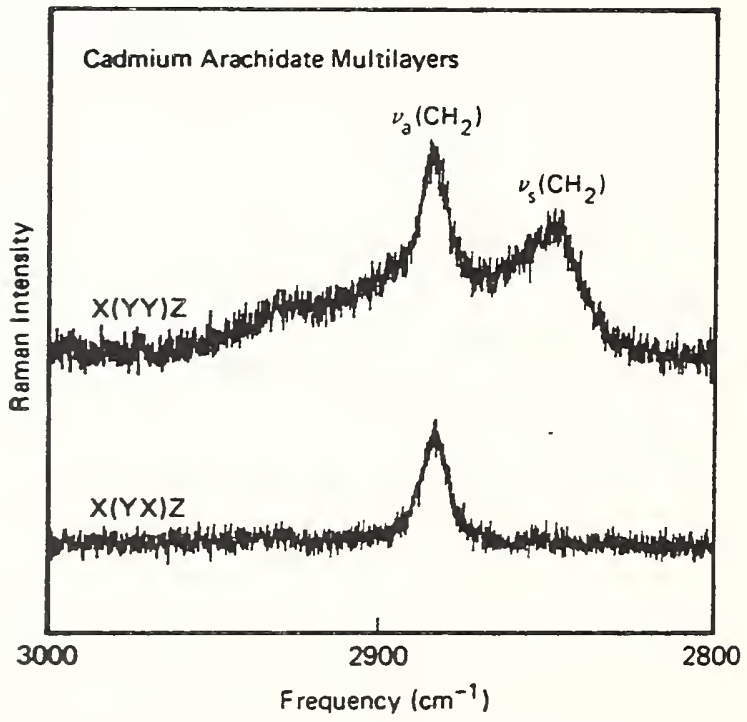

Fig. 5. Polarized Raman spectra of CdA multilayers in the $\mathrm{C}-\mathrm{H}$ stretching region.

The spectrum shown in Fig. 7 shows vibrations which are characteristic about tunneling in the zigzag structure of the backbone, between $1060 \mathrm{~cm}^{-1}$ and $1130 \mathrm{~cm}^{-1}$, which tells you how the chains are oriented. 


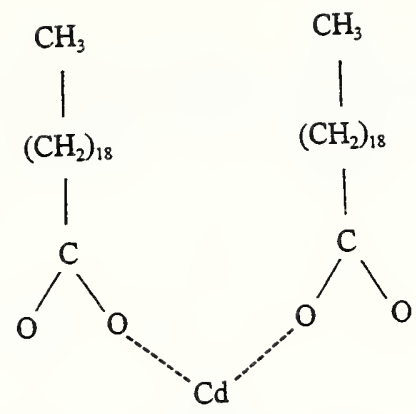

Fig. 6. Chemical structure of the CdA molecule.

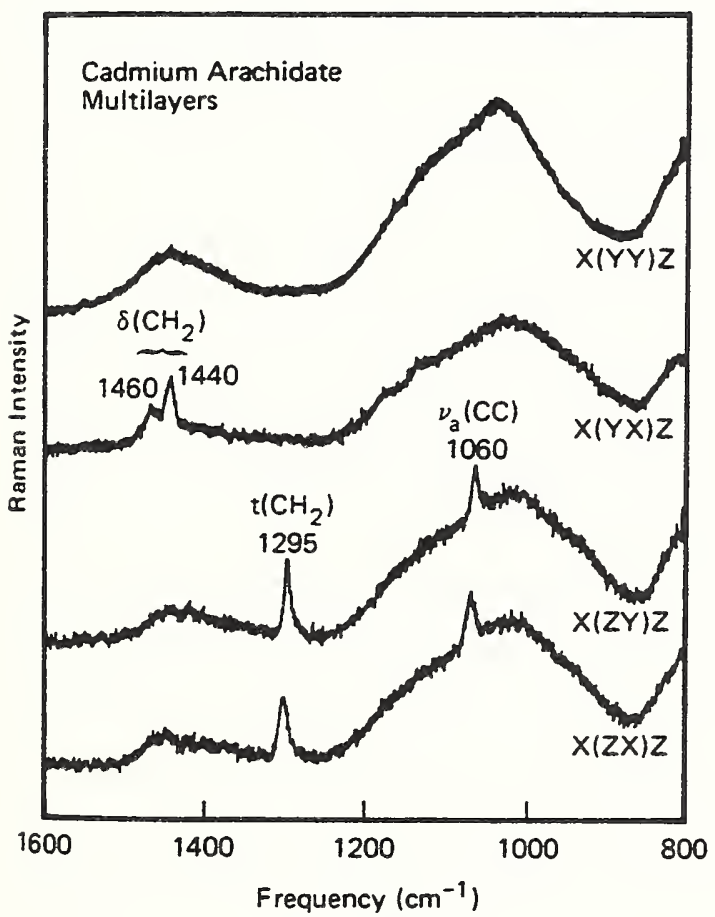

Fig. 7. Raman spectrum of CdA in the region of the C-C stretch and $\mathrm{CH}_{2}$ bending vibrations. The film had $8 \mathrm{CdA}$ layers (4 bi-layers) and its thickness was $20 \mathrm{~nm}$.

\section{Tailoring the Optical Field Intensity}

It is also possible and useful to shape, or tailor make, the optical field in a multilayer film. The example show at the top of Fig. 8 represents a mixed layer of poly(methyl methacrylate) (PMMA) with poly(styrene)(PS) on a quartz substrate. This mixed layer is thermodynamically unstable and, upon annealing, the components separate out into alternating layers of PS and PMMA thereby forming a diblock. This layered structure presents the possibility of shaping the optical intensity across the diblock for which the component layers have different indices of refraction (the indices for PMMA and PS are 1.54 and 1.62, respectively). The solutions to Maxwell's equations are shown in the middle of the figure
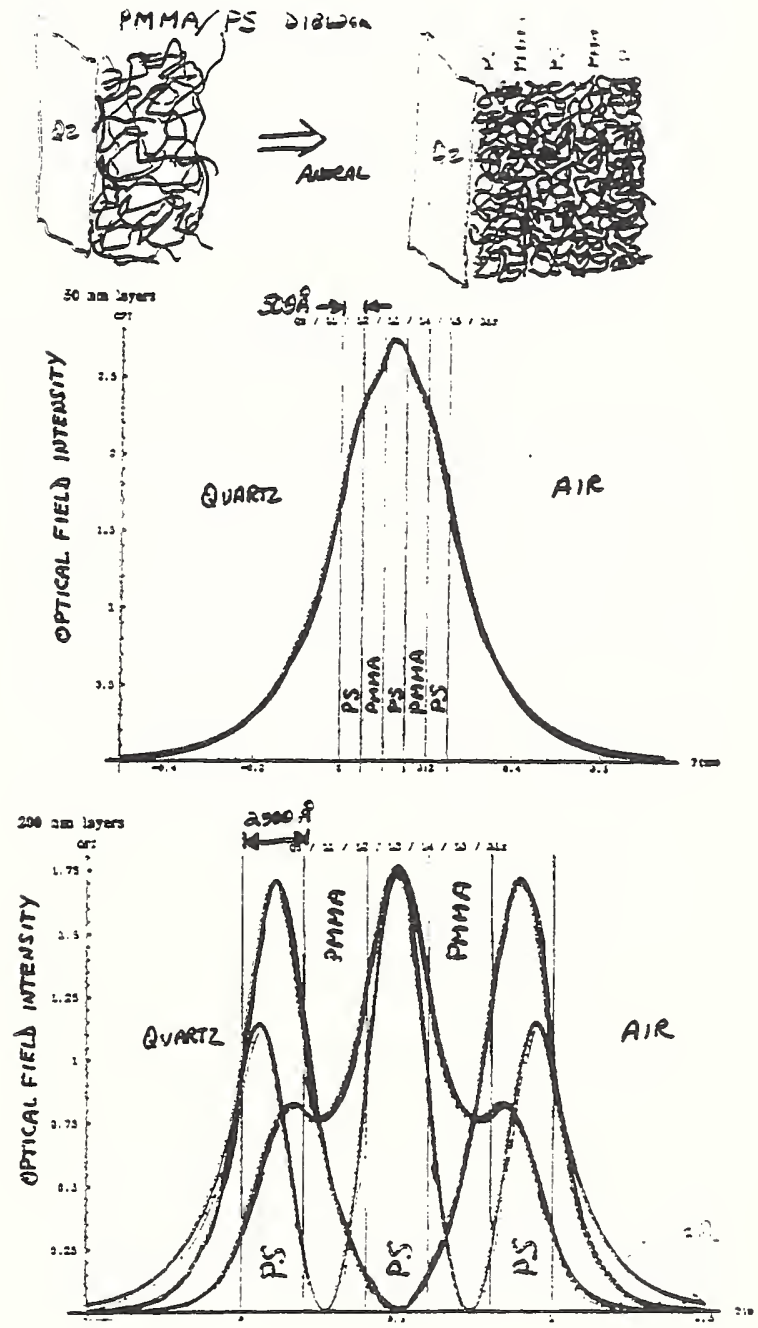

Fig. 8. Shaping the optical field intensity in a diblock. ( courtesy of Dr. N. Thomas, MIT)

for a stack of 5 layers each of which is $50 \mathrm{~nm}$ thick, while the bottom shows the field intensity for the same number of layers each of which, however, is $200 \mathrm{~nm}$ thick. Though appearing superficially the same as for a single homogeneous layer as shown in Fig. 2, the field intensity is actually markedly different. For the stack with layers of $50 \mathrm{~nm}$ thickness we note that the field intensity is no longer gaussian (cf. the $\mathrm{m}=0$ mode in Fig. 2) and similar differences exist for the stack in which the layer thickness is $200 \mathrm{~nm}$. The electric field intensity 
varies markedly in the various components of the high index and low index materials. This suggests that if one can dissolve individual molecules in the material comprising the high or low index layers, then one would have the possibility of generating Raman spectra from these molecules owing to the enhanced optical field in these layers. Figure 9 shows the results of a series of calculations for a 7 layer waveguide consisting of five alternating layers of PS and PMMA bounded by quartz.

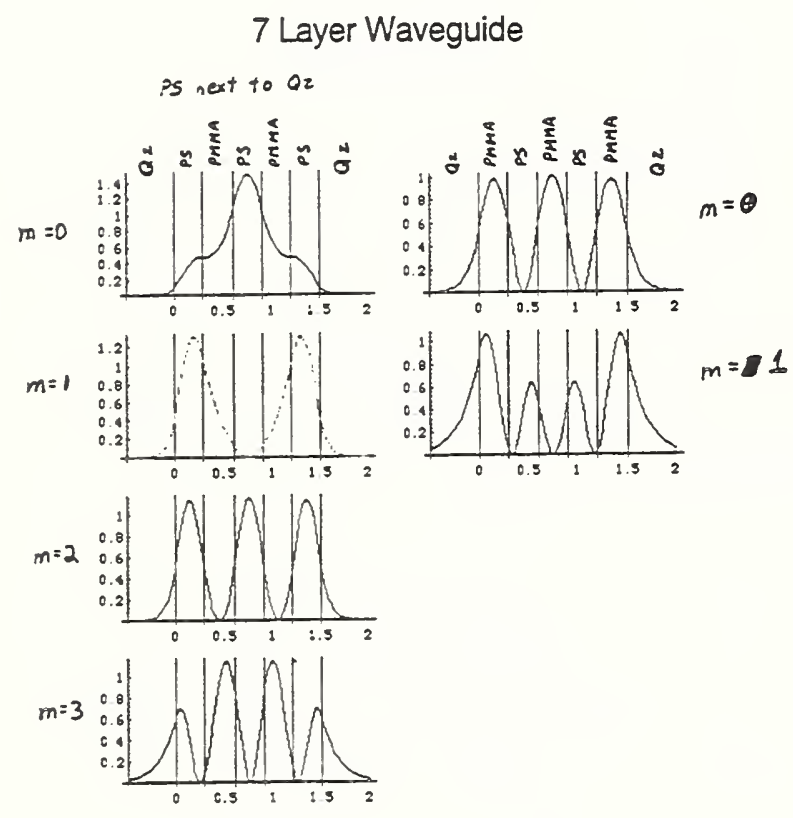

Fig. 9. Calculated field distribution in a seven layer waveguide composed of five alternating layers of PS and PMMA bounded by quartz. (Courtesy of C.G. Zimba, Polaroid Corp. and J. Chen, MIT.)

Particularly striking are the results for the case where the polystyrene layer is next to the quartz. Strong electric fields are seen to exist in the PS layers for the modes $m=0,1$ and 2 while for $m=3$ the field is stronger in the PMMA component. If the PMMA layer is next to the quartz the order is reversed. A test of these predictions is shown in Fig. 10 where the top trace is that obtained from the PMMA/PS mixture while the lower one shows the spectrum of the biblock in which the PMMA and PS components have separated to form their own layers. The same spectral features are present in both traces, but the lower one exhibits a much better signal to noise ratio and an increase in the strength of the PMMA band. These are attributed to the electric field enhancement in the layers.

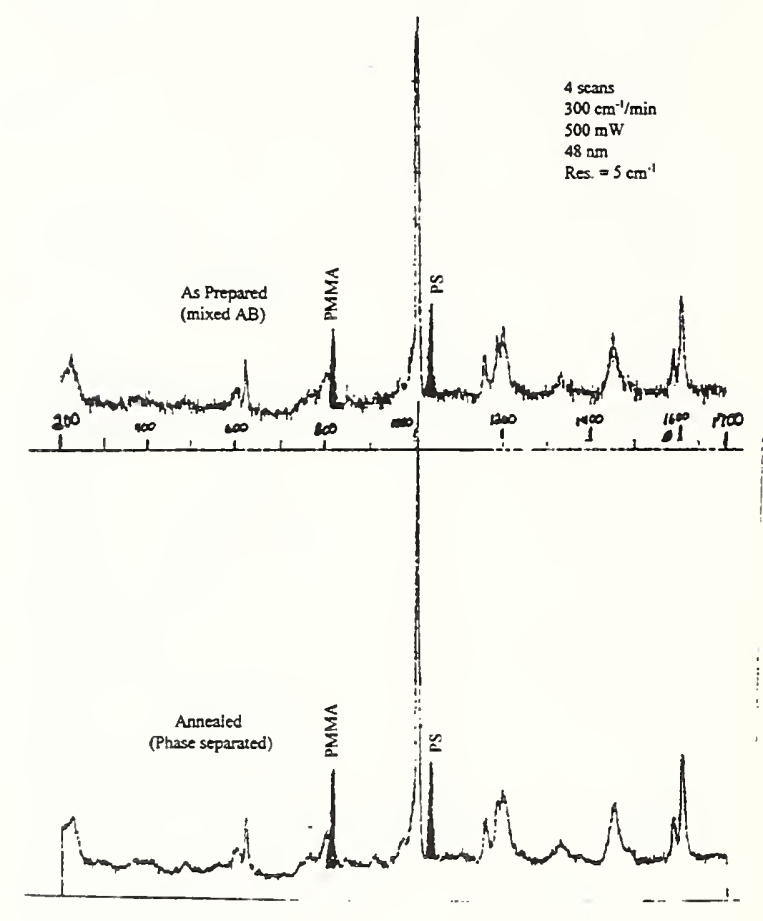

Fig. 10. Raman spectra of a PMMA and PS mixture (top) and the same material after annealing with attendant phase separation into alternating PMMA and PS layers (bottom). Both spectra were recorded under the same conditions.

\section{Use of Fiber Optics and Fourier Transform Techniques}

The reason why in the past Raman spectroscopy has had limited uses in polymer science is that many of these materials, as well as the additives, plasticisers, etc. fluoresce when excited with visible light. One of the solutions to this difficulty has been to shift the whole Raman excitation process into the near infrared and use a Fourier transform (FT) spectrometer to record the spectrum [7]. Figure 11 shows the common excitation wavelengths used to generate Raman spectra. While excitation with the red $\mathrm{Kr}^{+}$ laser radiation is in many cases helpful to suppress or even eliminate the fluorescence, the best success has been achieved with the Nd:YAG laser oscillating at $1.064 \mu \mathrm{m}$. The vibrational Stokes Raman spectrum then lies further in the near infrared, spanning the range 


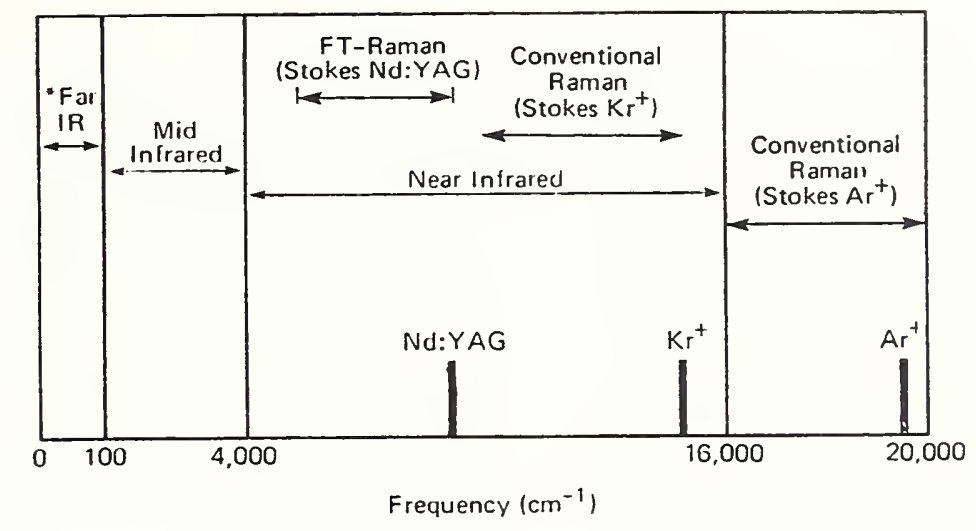

Not to scale

Fig. 11. Schematic diagram showing commonly used laser lines and the Raman spectra excited by them.

from $4000 \mathrm{~cm}^{-1}$ to slightly more than $9000 \mathrm{~cm}^{-1}$. Though dispersive infrared spectrometers cover this range, it has proven to be more advantageous to use a Fourier transform spectrometer to record the spectrum. For this reason the technique is now commonly referred to as FT-Raman spectroscopy. Taking the vibrational bands to span a region of $4000 \mathrm{~cm}^{-1}$, we see that the upper limit of the Stokes Raman bands excited by the argon laser $(\lambda=514 \mathrm{~nm})$ is about $15,400 \mathrm{~cm}^{-1}$, or $648 \mathrm{~nm}$, while for the krypton laser $(\lambda=647 \mathrm{~nm})$ it is nearly 11,400 $\mathrm{cm}^{-1}$, or $873 \mathrm{~nm}$. While for many systems the fluorescence is minor when krypton laser excitation is used it is still a problem for many polymers. The Nd:YAG laser, however, oscillates at a sufficiently long wavelength that it has become the choice excitation source for Raman spectra which otherwise would be overwhelmed by sample fluorescence.

Figure 12 shows the experimental arrangement that one would initially come up with if the visible argon or krypton laser were replaced by a

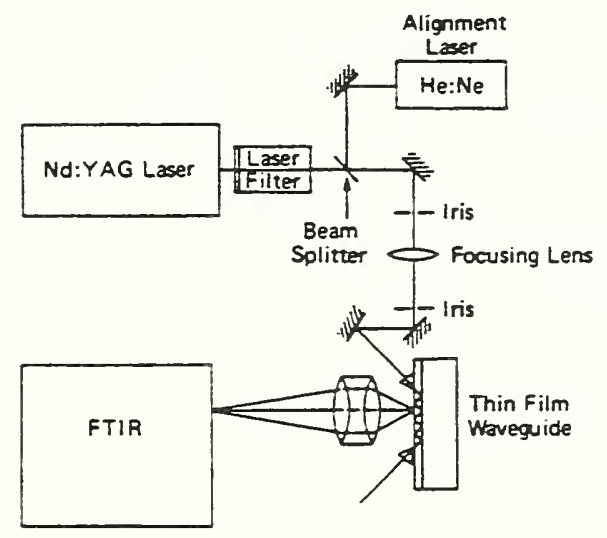

Fig. 12. Simple experimental arrangement for an FTRaman experiment with Nd:YAG laser excitation.
Nd:YAG laser and the slit spectrometer by a FTspectrometer operating in the near infrared. Since the Nd:YAG laser operates in the infrared, a visible $(\mathrm{He}-\mathrm{Ne})$ laser is used to facilitate the optical alignment. The source of Raman radiation is, as before, the line-like streak in the thin film which is imaged onto the entrance aperture of the FT-spectrometer. The resulting performance of such an arrangement is demonstratedin Fig. 13 which shows the spectrum of polystyrene. The top spectrum is

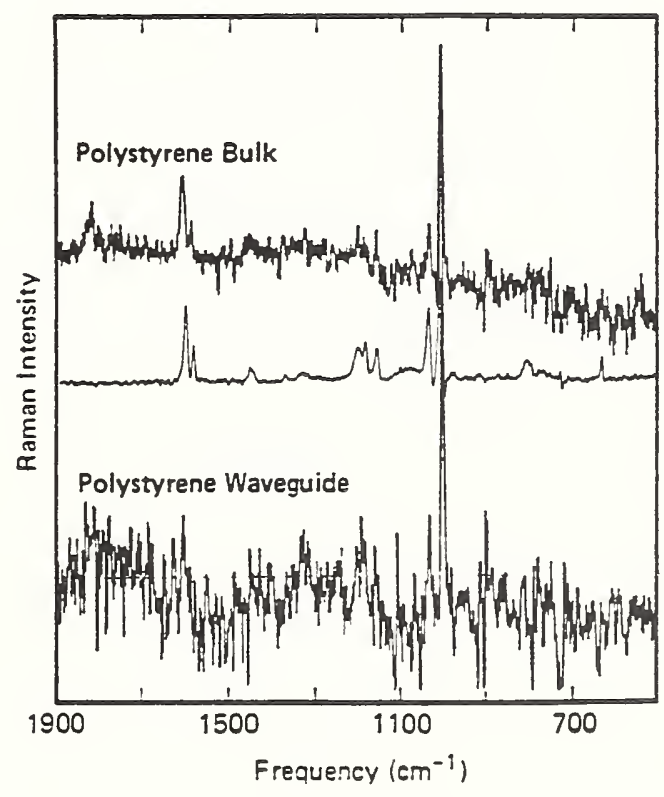

Fig. 13. Raman spectra of polystyrene obtained with the arrangement shown in Fig. 12.

that obtained from the bulk material while the spectrum on the bottom is that from a polystyrene waveguide of thickness $2 \mu \mathrm{m}$. Comparing the spectrum of the bulk with that 
generated with visible excitation from a guided wave film (see Fig. 3, bottom spectrum) we note the striking reduction in the signal to noise ratio $(\mathrm{S} / \mathrm{N})$. This is to be expected since the intensity of the Raman scattering varies inversely with the fourth power of the wavelength of the exciting light. Thus, going from $\lambda_{\text {Argon }}=514 \mathrm{~nm}$ to $\lambda_{\mathrm{Nd}: \mathrm{YAG}}=1.064 \mu \mathrm{m}$ entails a reduction in the intensity of the Raman signal by a factor 18.4. More disturbing, however, is the fact that the PS waveguide spectrum (Fig. 13, bottom) is very much worse than that of the bulk, showing only the strong band at $1,000 \mathrm{~cm}^{-1}$, the others being buried in the noise. The cause for this lies in the method of transferring the Raman scattered radiation to the FT-spectrometer. This problem is illustrated in Fig. 14, which shows the plane of the (circular) entrance aperture of the spectrometer and the image of the light streak in

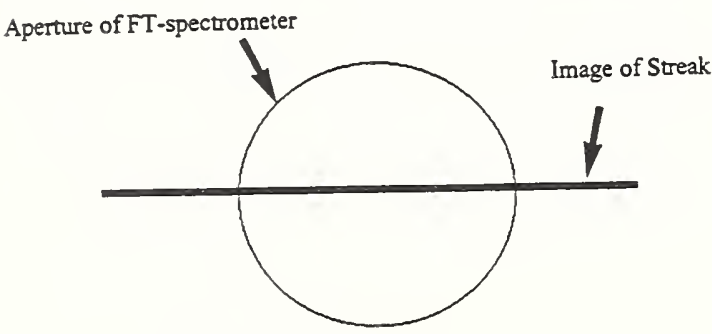

Fig. 14. Difficulty of transferring the line-like Raman source to an FT-spectrometer (schematic).

the film. A significant portion of the light contained in this image does not enter the spectrometer and the full throughput advantage of the FT-spectrometer system is not realized. This problem was solved by using a fiber optic

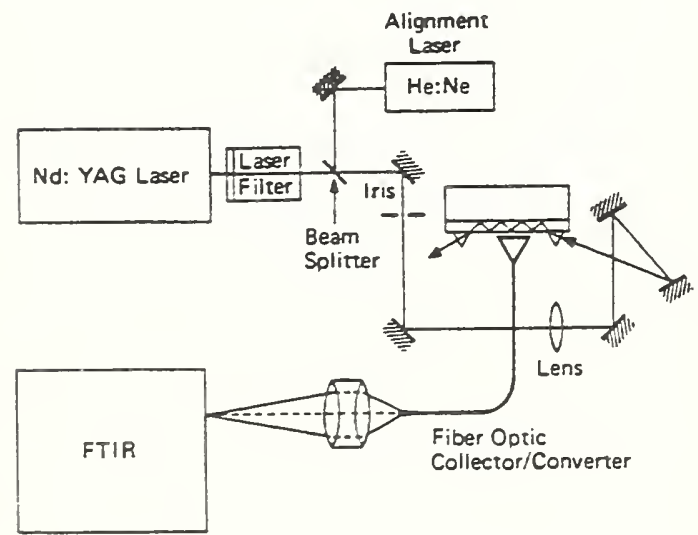

Fig. 15. Use of a fiber optic collection/converter system to transfer the Raman light streak from a thin film to an FT-spectrometer [8]. bundle with a rectangular area on one end and a circular one at the other to collect the light from the light streak, convert it to a circular form and transfer it to the FT-spectrometer, as indicated in Fig. 15 [8]. The spectrum from a polystyrene waveguide obtained with such collection system is shown as the middle trace in Fig. 13, which represents a major improvement not only over that shown in the bottom trace but even over the spectrum of the bulk (top trace) and is more like the one shown in Fig. 3 recorded with a slit spectrometer.

Realistically, however, the Raman spectrum of polystyrene is not plagued by fluorescence, as is evidenced by the spectrum shown in Fig. 3 which was generated with argon laser excitation. A more convincing demonstration of the elimination of fluorescence in Raman spectra is that offered by Fig. 16, which shows the Raman spectra of a cellulose acetate film with and without embedded fluorescing dye molecules.

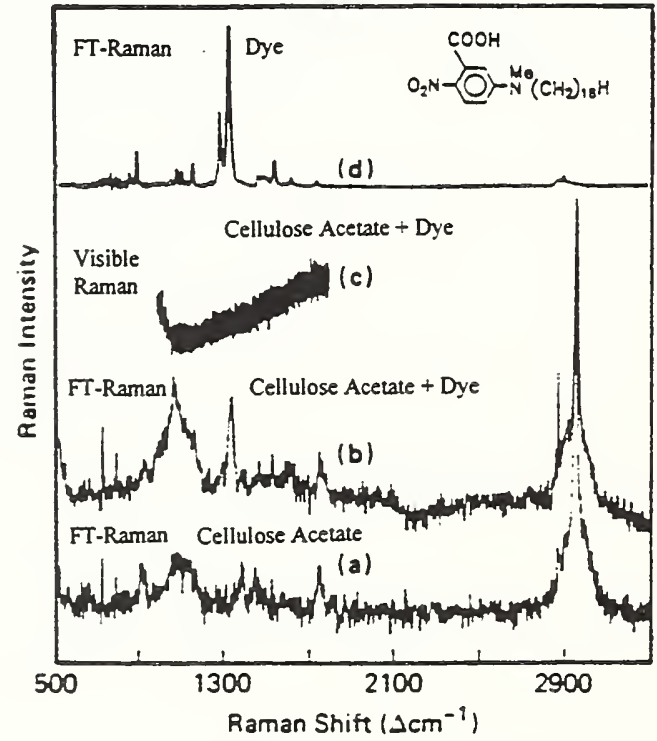

Fig. 16. Raman spectra of cellulose acetate. (a) Waveguide FT-Raman spectrum of a cellulose acetate film. Waveguide FT-Raman spectrum of of a composite film of cellulose acetate with 4\% 2-nitro-5(N-methyi-N-octadecylamino)benzoic acid. (d) Waveguide FT-Raman spectrum of 2-nitro-5-(N. methyl-N-octadecylamino)benzoic acid powder. (c) Raman spectrum of a composite film of cellulose acetate with $4 \%$ 2-nitr-5-(N-methyl-Noctadecylamino)benzoic acid with $488.0 \mathrm{~nm}$ excitation. The films were $4.5 \mu \mathrm{m}$ thick and on quartz substrates. The waveguide spectra were recorded with approximately $1 \mathrm{~W}$ of laser power and $4.5 \mathrm{hr}$ measurement time while the powder spectrum was recorded with $400 \mathrm{~mW}$ of laser power in $35 \mathrm{~min}$ [8]. 
The spectra displayed in Fig. 16 give evidence of the superiority of the FT-Raman tchnique when dealing with fluorescing materials. The spectrum of the bulk dye itself (d) is clean and free of any traces of fluorescence and the strong vibrational mode near $1,300 \mathrm{~cm}^{-1}$ clearly stands out in the spectrum of the weak solution of the dye in the acetate film (b). It is also obvious that excitation with visible laser light (c) is incapable of generating any Raman spectrum.

So, we see that with these changes in the technique of Raman spectroscopy: moving the excitation wavelength from the visible to the infrared region, using a Fourier transform spectrometer instead of a traditional slit spectrometer, and employing fiber optics to couple the Raman scattered light into the FTspectrometer, the problem of fluorescence that has prevented the use of Raman spectroscopy in polymer science has now been overcome.

The evolution of the different aspects of waveguide Raman spectroscopy presented here is summarized in Fig. 17. Starting with a single thin $(\mathrm{d}<1 \mu \mathrm{m})$ layer on a substrate we saw how one can probe the waveguide material. The evanescent wave allows probing of molecular monolayers deposited on the waveguide surface,

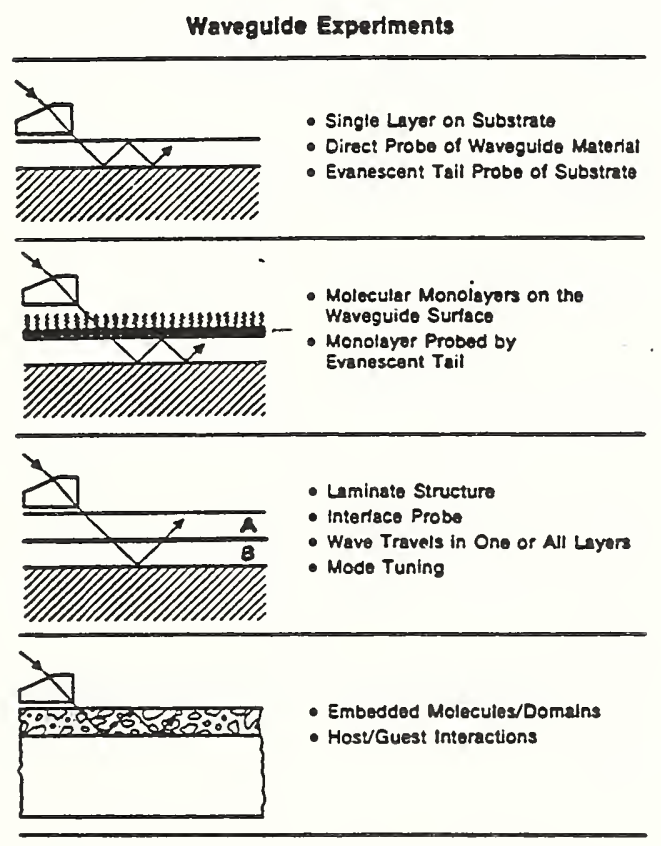

Fig. 17. Summary of possible waveguide Raman experiments.

the monlayers being as thin as approximately $2.7 \mathrm{~nm}$ when resonance effects enhance the intensity of the Raman scattering or $20 \mathrm{~nm}$ in the case of non-resonant Raman excitation. It is possible to investigate laminate structures in which the evanescent wave travels in one or all layers. In such, as well as the previously mentioned, cases it is also possible to mode tune and shape the electric field intensity to enhance the strength of the Raman signal. Lastly, it is possible to study embedded molecules and probe host/guest interactions. All of these variations of waveguide Raman spectroscopy have been developed for purposes of industrial applications.

\section{Backbone Conformation in Ceramic Precursor Polymers}

Another area in which Raman spectroscopy of polymers is of industrial interest is the investigation of ceramic prcursors. Poly(di-nalkylsilanes) (Fig. 18) has been known since the 1950 s, conveniently in the liquid form. The structures of these system was presumed to be symmetric, the reason being that, for the solid, they have long wavelength absorptions in the ultraviolet. This absorption was suggested to be due to conformational locking of the polysinane backbone into a specific configuration (perhaps planar zigzag) that is different from that

\section{Poly(di-n-alkylsilanes)}

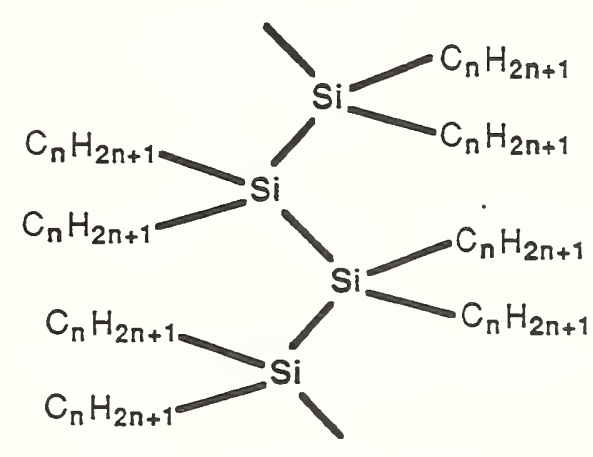

- Conjugated sigma-bond chain

- Backbone conformation sensitive to
- pressure
- temperature
- solvent

Fig. 18. Structure of Poly(di-n-alkylsilanes). 
normally observed in solution or amorphous films and that this locking is caused by a crystallization of the $n$-hexyl substitutent groups into a paraffin-like matrix. This suggested that they may be useful as ceramic precursors. Their backbone conformation is sensitive to pressure, temperature, and solvent [9], and one would expect to observe attendant changes in the orbital energies and hence spectral properties. For these, and other, reasons it was thought appropriate to study these interesting systems by Raman spectroscopy. Figure 19 shows a plot of the ultraviolet absorption spectrum for a range of temperatures from $18^{\circ} \mathrm{C}$ to $45^{\circ} \mathrm{C}$. We note that the intensity of the band at ca. $315 \mathrm{~nm}$ increases

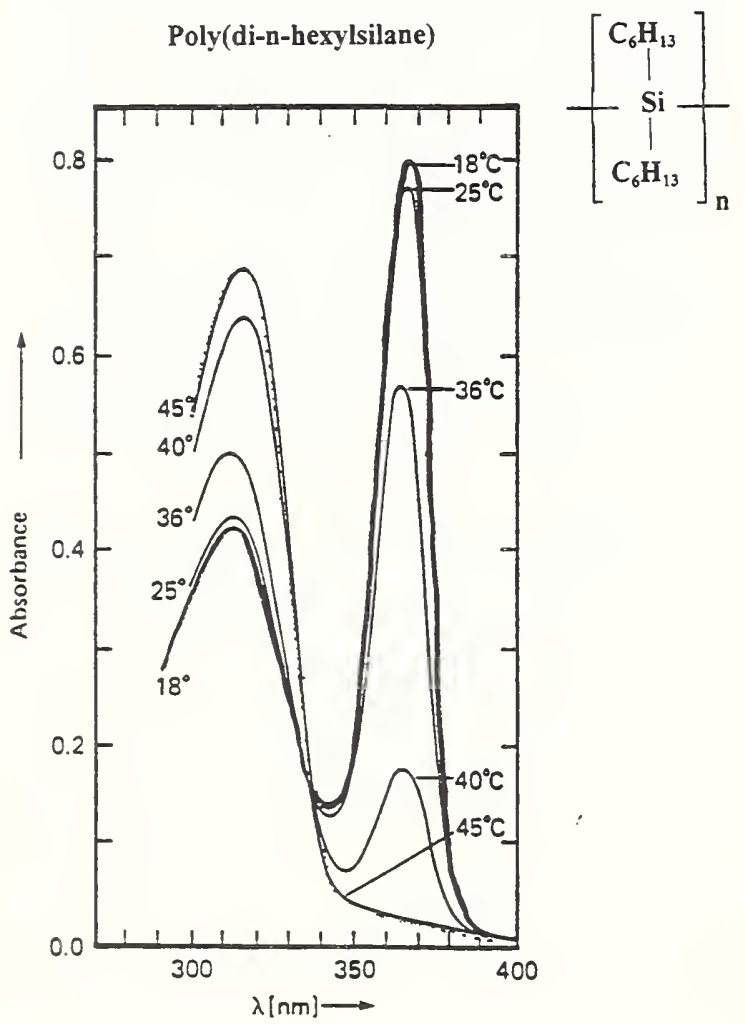

Fig. 19. Temperature dependence of the ultraviolet absorption spectrum of poly(di-n-hexylsilane).

with temperature while that of the $365 \mathrm{~nm}$ band decreases. The question is what is the mechanism that causes this shift in the intensity of the ultraviolet spectrum? One of the first methods used to investigate this problem was to use Raman scattering. Fig. 20 shows the Raman spectrum of this material at different temperatures. The lower portion of the figure displays the conventional Raman spectrum excited by the green argon laser line and recorded with a slit spectrometer at $15^{\circ} \mathrm{C}$ and $46^{\circ} \mathrm{C}$. For this material one would expect that with argon $514 \mathrm{~nm}$ line excitation one would be far removed from resonance. It turns out, however, that with this wavelength we are already in a pre-resonance region. The assignments of the observed bands to the various vibrational modes was straight forward. The upper part of the figure displays the same region of the spectrum recorded with an FT-Raman

Poly(di-n-hexylsilane)

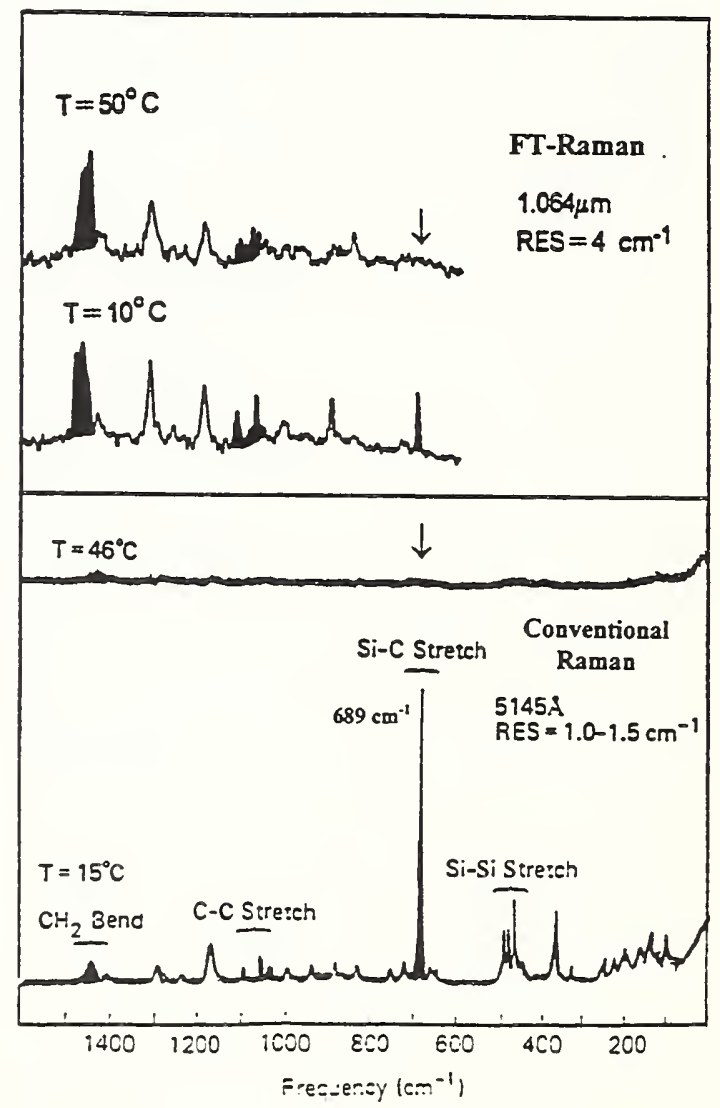

Fig. 20. Raman spectrum of poly(di-n-hexylsilane) at ambient and elevated temperatures.

setup at temperatures of $10^{\circ} \mathrm{C}$ and $50^{\circ} \mathrm{C}$. We note the very pronounced changes in the intensities of the vibrational bands. The $689 \mathrm{~cm}^{-1}$ band of the Si-C stretching mode of the Si-C backbone completely disappears upon heating to $46^{\circ} \mathrm{C}$ while the intensities of the $\mathrm{CH}_{2}$ bends and $\mathrm{C}-\mathrm{C}$ stretch modes increase. Also to be noted arew the relative intensities between the $\mathrm{Si}-\mathrm{C}$ stretch mode and the $\mathrm{C}-\mathrm{C}$ stretch and $\mathrm{CH}_{2}$ bend modes, which should appear with much greater intensity, more representative of the 


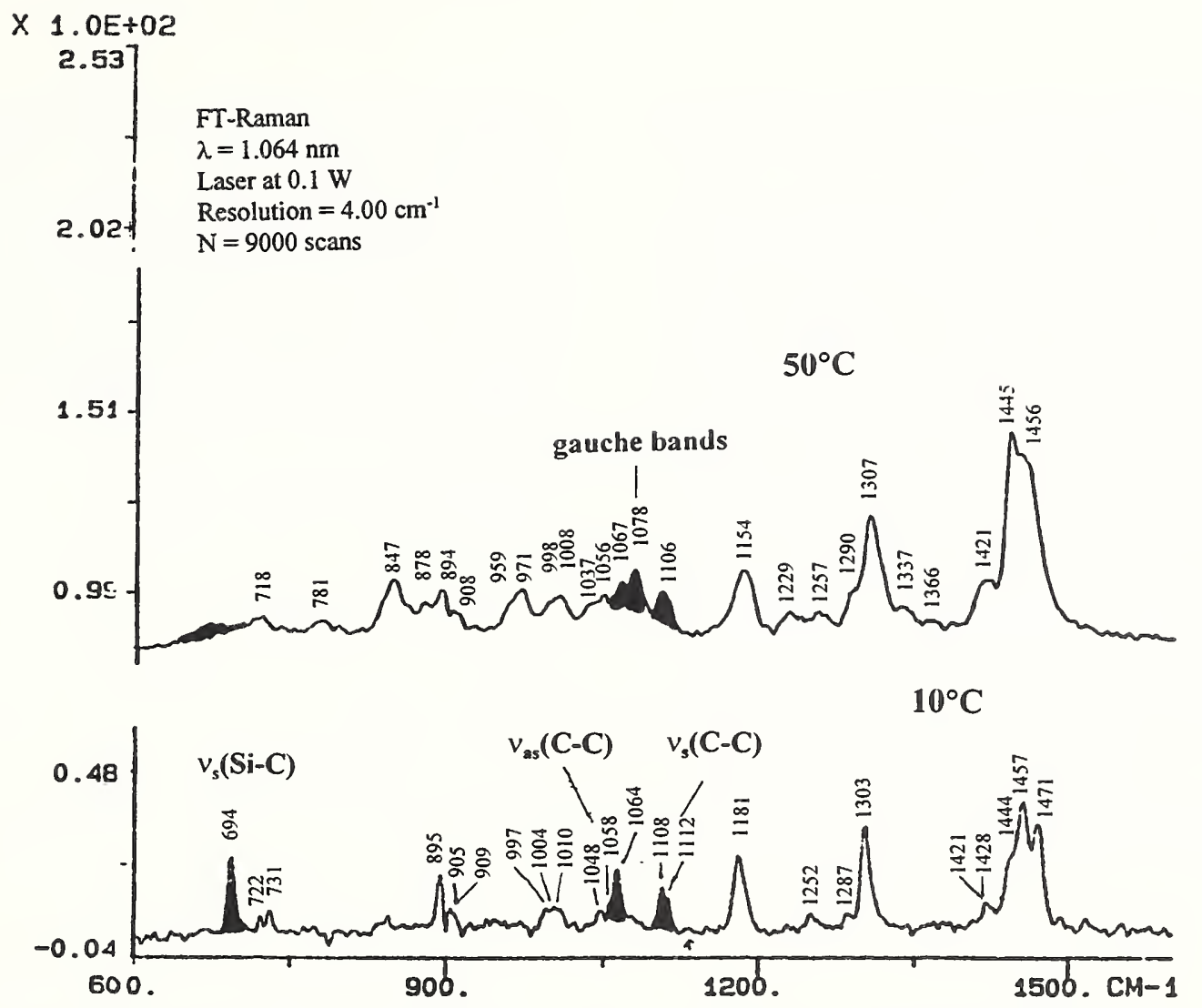

Fig. 21. Assignments of Raman bands of poly(di-n-hexylsilane) in the region $600-1500 \mathrm{~cm}^{-1}$.

chemical concentration of the methyl groups. To avoid any difficulties in the interpretation of this behavior associated with the pre-resonance effect the experiment was repeated with near infrared excitation, the result of which is shown in the top portion of the figure. Here the spectra were excited by the $1.064 \mathrm{~nm}$ line of the $\mathrm{Nd}$ :YAG laser. The relative intensities of the $\mathrm{Si}-\mathrm{C}$ mode and the $\mathrm{C}-\mathrm{C}$ and $\mathrm{CH}_{2}$ modes is much more realistic.

Figure 21 shows in more detail the Raman spectrum when recorded with infrared excitation, i.e., outside the region of resonance. One can now identify the gauche bands at $1078^{\mathrm{cm}-1}$ in the high temperature $\left(50^{\circ} \mathrm{C}\right)$ spectrum whereas at the lower temperature the symmetric and anti symmetric $\mathrm{C}-\mathrm{C}$ stretching vibrations are clearly separated. The symmetric Si-C stretching vibration at $694 \mathrm{~cm}^{-1}$ is broadened out into a very weak band. This band is thus very sensitive to conformation and one needs to come up with a model not only for a good understanding of the Raman spectrum but one which is also capable of dealing with the temperature dependence of thermophysical properties and calorimetry. At temperatures below $40^{\circ} \mathrm{C}$ poly(di-n-hexylsilane) back bone has a regular planar zigzag structure (see Fig. 22). This conclusion is based on the
POLY(DIHEXYLSILANE)<smiles>[R][Si]([R])(C)[Si]([R])([R])[Si]([R])([R])[Si]([R])([R])[Si]([R])([R])C</smiles>

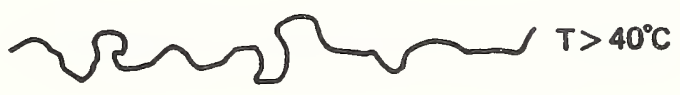

\section{Transition is Reversible}

Fig. 22. Structural models of poly(di-n-hexylsilane) at different temperatures.

study of the Raman, as well as the infrared spectrum of this material and the comparison of the prediction of different models with the observed spectra. Above $40^{\circ} \mathrm{C}$ the structure is highly random. It is the Raman spectrum that is particularly useful in obtaining information about the extent of the conformational changes 
that are going on. The $694 \mathrm{~cm}^{-1}$ wave number band is the strongest indication of the zigzag structure of the Si-backbone.

Going now from the hexylsilane to the poly(di-n-pentylsilane) and looking at the region of the Si-C stretching vibration at $694 \mathrm{~cm}^{-1}$ in the Raman spectrum at ordinary conditions, i.e., at a pressure of 1 atmosphere, shown at the top of Fig. 23, one would think that there is no zigzag structure in this system. Other information, however, such as that obtained from $\mathrm{x}$-ray diffraction, suggests that there is a helical structure. At a pressure of $9 \mathrm{kbar}$, however, the backbone has undergone a conformational change to that of a planar zigzag structure. At a still higher pressure, $20 \mathrm{kbar}$, the planar zigzag

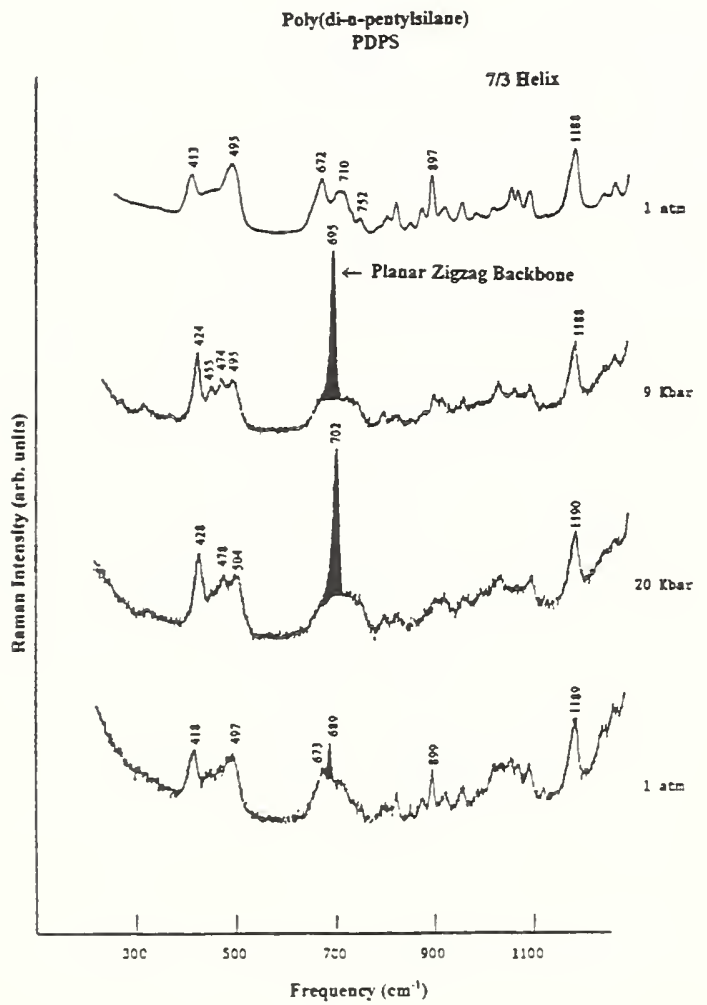

Fig. 23. Raman spectrum of poly(di-n-pentylsilane) at various pressures.

structure persists. Upon relaxing the pressure back to $1 \mathrm{~atm}$ (bottom spectrum) the system does not quite revert back to its starting structure. There is hysteresis as indicated by the band at $689 \mathrm{~cm}^{-1}$; a small amount of the material persists in the planar zigzag conformation. In light of this information we repeated our ultraviolet absorption studies but with the sample under pressure. Fig. 24 shows the results. The material is seen to be piezochromic; the absorption band peaked at approximately 317 $\mathrm{nm}$ in the spectrum recorded at 1 atm shifts to near $365 \mathrm{~nm}$ at $4 \mathrm{Kbar}$ and then back to about $358 \mathrm{~nm}$ at $14 \mathrm{Kbar}$. The disordered structure changes over into an ordered one at higher pressure. Figure 25 gives an overview summary of the work done on the polysilanes.

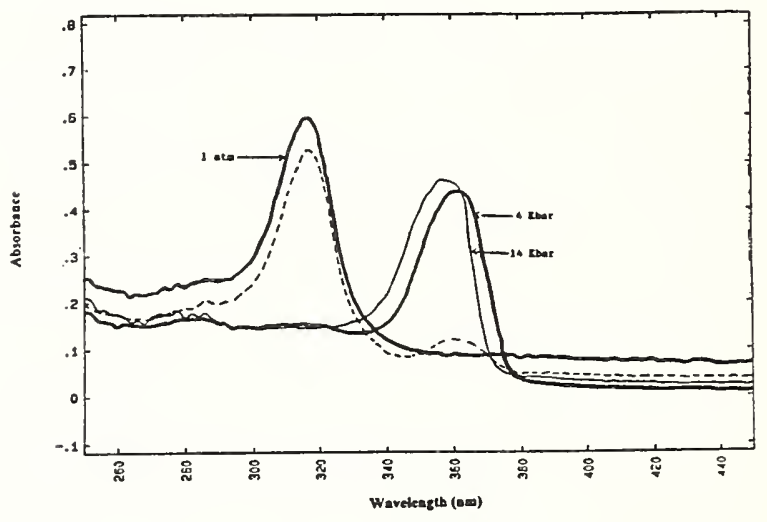

Fig. 24. Piezochromic effect in the ultraviolet absorption spectrum of poly(di-n-pentylsilane).

\begin{tabular}{|c|c|c|c|}
\hline \multicolumn{4}{|c|}{ Polyailsione Uand is This Work } \\
\hline & 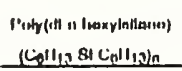 & 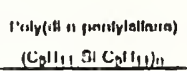 & 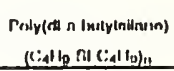 \\
\hline 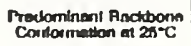 & Trrne plnurst & $7 / 3$ thellx & 73 Hindx \\
\hline Promery UV promk & $370 \mathrm{~nm}$ & $318 \mathrm{~mm}$ & $310 \mathrm{~min}$ \\
\hline 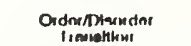 & $42 C$ & $0070^{\circ} \mathrm{C}$ & oo ror: \\
\hline Flloct of Pronturn & 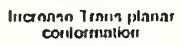 & 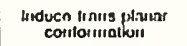 & $\begin{array}{l}\text { Induco hans plinutu } \\
\text { conlormatiour }\end{array}$ \\
\hline 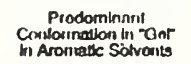 & No "goll lormod & Tinna plannt & Trana-plnisur \\
\hline
\end{tabular}

Fig. 25. Summary results obtained for the structural studies performed on polysilanes.

As a final example we present some work done on polysilaethylene $\left(\left[\mathrm{SiH}_{2} \mathrm{CH}_{2}\right]_{\mathrm{n}}\right.$ ) (PSE). In this system the backbone is formed by silicon and carbon atoms. It may be viewed as a hybrid of the prototypical all-organic polymer, polyethylene $\left[\mathrm{CH}_{2} \mathrm{CH}_{2}\right]_{\mathrm{a}}(\mathrm{PE})$, and the allinorganic polysilane discussed above. In addition to the interest in PSE as an analog of PE and the parent member of an emergent family of poly(silylenemethylenes), this polymer is also of interest as a precursor to silicon carbide, an important ceramic material. Preliminary studies of PSE by thermal analysis, NMR, and ab initio calculations have suggested a relatively low torsional barrier for the PSE backbone; the 
presence of a crystalline phase below $0^{\circ} \mathrm{C}$, in which the chains appear to assume an all-trans conformation; and thermal decomposition above ca. $200^{\circ} \mathrm{C}$ to produce stoichiometrically nearly pure SiC. The conversion efficiency from PSE to $\mathrm{SiC}$ is about $90 \%$, which is very high compared to what normal ceramic precursor conversion factors are. For these reasons we undertook a detailed study of the crystallization and the solid-state structure of PSE vibrational spectrum by Raman and infrared spectroscopy, and other techniques [10].

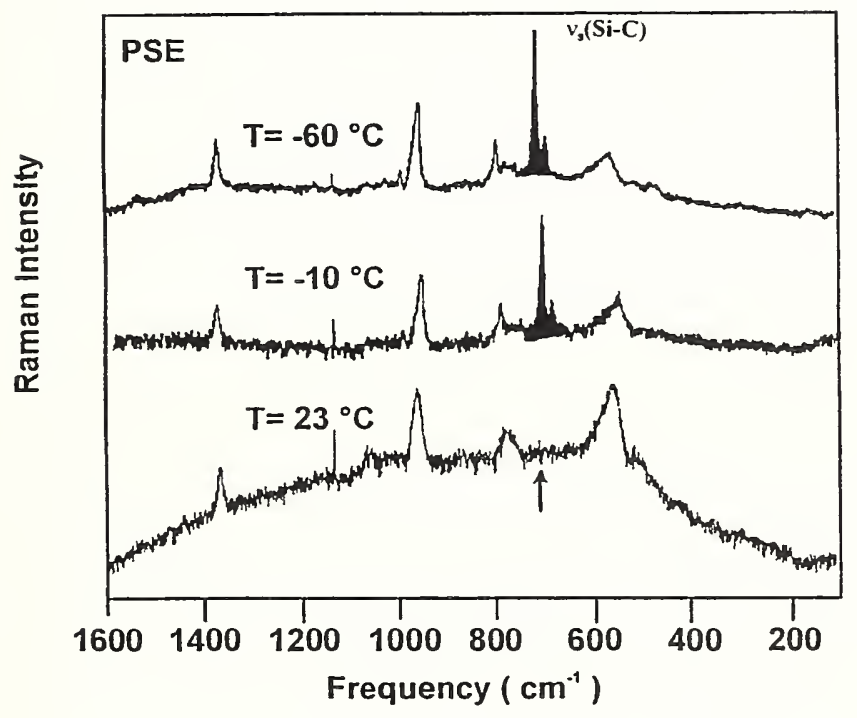

Fig. 26. Raman spectrum of PSE from $100 \mathrm{~cm}^{-1}$ to $1600 \mathrm{~cm}^{-1}$ [10].

Figure 26 shows the Raman spectrum of PSE from $200 \mathrm{~cm}^{-1}$ to $1600 \mathrm{~cm}^{-1}$ at three different temperatures. Most noticeable is the absence of the symmetric Si-C stretching vibration at $706 \mathrm{~cm}^{-1}$ in the room temperature spectrum. At this temperature the polymer is essentially a rubber, a viscous liquid which crystallizes upon cooling. The crystallization/melting occurs at $-20^{\circ} \mathrm{C}$, bringing the $\mathrm{Si} / \mathrm{C}$ backbone into an alltrans (planar zigzag) configuration. At this time, however, this process is not well understood and requires further study.

\section{Summary}

The work described briefly in the foregoing examples of the use of Raman spectroscopy in five different areas of polymer chemistry have all been motivated by industrial interests and needs. This work was done while the author was associated with the IBM Almaden laboratories and illustrates the advances in the Raman spectroscopic technique that have taken place during the last few years. The progress has been such that method is now becoming incorporated in industrial process streamlines for monitoring the quality of the production of polymers. Another area, one which was not discussed here, is the use of fiber optics in Raman spectroscopy for applications in an aqueous environment. Many industrial fabrication processes which have heretofore relied on the use of organic solvents are gradually changed over to the use of environmentally benign aqueous solvents. Because the Raman spectrum of water is relatively weak, it is easier to discern the species to be monitored than with organic solvents.

\section{References}

1. J.D. Swalen, J. Phys. Chem. 83, 1438-1445 (1979).

2. J.F. Rabolt, R. Santo, and J.D. Swalen, Appl. Spectrosc. 33, 549-551 (1979).

3. J.F. Rabolt, N.E. Schlotter, and J.D. Swalen, J. Phys. Chem. 85, 4141 -4144 (1981).

4. J.D. Swalen, M. Tacke, R. Santo, K.E. Rieckhoff, and J. Fischer, Helv. Chim. Acta 61, 960-977 (1978).

5. J.P. Rabe, J.D. Swalen, and J.F. Rabolt, J. Chem. Phys. 86, 1601-1606 (1987).

6. T.C. Damen, S.P.S. Porto, and B. Tell, Phys. Rev. 142, 570 (1966).

7. See, for example, Fourier Transform Raman Spectroscopy: From Concept to Experiment, B. Chase and J.F. Rabolt, Eds. (Academic Press, 1994) and references cited therein.

8. C.G. Zimba, V.M. Hallmark, S. Turrell, J.D. Swalen, and J.F. Rabolt, J. Phys. Chem. 94, 939-943 (1990).

9. R.D. Miller, D. Hofer, J. Rabolt, and G.N. Fickes, J. Am. Chem. Soc. 107, 2172-2174 (1985).

10. M.-W. Tsao, K.-H. Pfeifer, J.F. Rabolt, D.B. Holt, B.L. Farmer, L.V. Interrante and Q. Shen, Macromolecules 29, 7130-7135 (1996). 


\section{Discussion}

Q If one were to use forward scattering rather than the ninety degree scattering configuration then the Raman lines themselves would be guided in different modes due to the refractive index and wavelength differences.

A Yes, this is a good point. One could couple out the light through the second prism and we actually did that, using a CCD detector to see the beams directly. Actually this is a very good idea, but we have not taken these experiments that far. At the time we were more concerned with determining the field intensity to compare it with that predicted by calculation. This is, however, not easy, since the nice separation in the beams predicted by the calculations is in fact not quite possible in the actual case since, as the theoretical curves show (see Figs. 8 and 9), the optical field intensity is not sharply delineated by the layer boundaries. Your suggestion is, however, a very good one.

Q Are these fields in the layers strong enough to give rise to Stark effects? The fields needed would be of the order of $10^{4}$ to $10^{5}$ volts $/ \mathrm{cm}$.

A This certainly depends on the input power but we have no information about this and we have not considered this possibility. I estimate, however, that the field intensity, which varies as the square of the electric field strength, is about $10^{5}$ times greater than what is present in a laser beam. These are $\mathrm{cw}$ experiments but experiments have also been done by others using pulsed laser beams in waveguides. The main problem that one has to be concerned about is the decomposition of the material at these intensities.

Q Why don't you couple the light out of the second prism and use it?

A As was already pointed out in an earlier question, if one did that one could see the Raman shifts produced by the individual layers of the multilayer stack since the beams that emerge from the second prism diverge and thus allow a spatial differentiation. In this way one could access the individual modes by projecting the fan of the emerging beams on a screen. The Raman shifts produced in the different layers would be different since the layers are made of different materials. However, what we do now, in order to do Raman spectroscopy, is what I described in the talk. 


\title{
Raman Spectroscopy of Semiconductor Heterostructures: Phonons and Excitons as Probes of Interfaces
}

\author{
Daniel G. Gammon \\ Naval Research Laboratory \\ Washington, DC 20375
}

\begin{abstract}
The layered structure GaAs/AlAs is investigated by photoluminescence-, photoluminescence excitation-, and Raman spectroscopy to study the characteristics of the layer interface. GaAs/AlAs interfaces are shown to be non-planar, with monolayerhigh islands having lateral extent from $20 \mathrm{~nm}$ to several hundred nm, forming large interruptions in the ideal interface flatness. There is an additional small scale $(<20$ $\mathrm{nm}$ ) structure within these large islands. These are modeled by an $\mathrm{Al}_{\mathrm{x}} \mathrm{Ga}_{1-x} \mathrm{As}$ alloy monolayer located on each side of the interface boundary. Near-field optical techniques are used to record and study Raman spectra from single quantum dots. By means of this technique the lateral extent of quantum dots can be determined.
\end{abstract}

\section{Introduction}

Raman scattering plays an important role in providing data for the understanding of semiconductor heterostructures. These are artificial systems, specially prepared to perform specified tasks in semiconductor electronics. In Raman scattering by solids one is able to probe the following types of elementary excitations: scattering by impurities, by electrons (plasmons, etc.) and, especially, by phonons as well as excitons. In the following we will focus on the scattering by phonons and excitons.

The layered structure GaAs/AlAs provides a prototype for semiconductor heterostructure systems. Such systems are now intensely studied in view of their potential technological importance. What is needed are data to understand their optical, electronic, and vibrational properties, and Raman spectroscopy is especially well equipped to meet these needs. Raman spectroscopy is also capable of furnishing information about the structural disorder at the interfaces between the $\mathrm{GaAs}$ and AlAs layers. Here the phonons and excitons serve as important probes of the interface roughness.

\section{Phonons in GaAs/AlAs Quantum Well Structures}

In this system we deal with acoustic phonons and optical phonons. The layered structure of this system provides for a slightly different point of view regarding the acoustic phonons, namely the folded acoustic phonons, a concept imposed by the periodicity of the layers [1]. Similarly, the optical phonons in heterostructures are the interface phonons and, especially, the confined phonons.

In the GaAs and AlAs bulk materials the dispersions of the (longitudinal) optical phonons (LO) with wave vector are shown at the top of Fig. 1. The bottom part of Fig. 1 depicts the situation for the $\mathrm{Al}_{\mathrm{x}} \mathrm{Ga}_{1-\mathrm{x}}$ As material. There are now two $\mathrm{LO}$ phonons, $\mathrm{LO}_{1}$ and $\mathrm{LO}_{2}$, whose energies lie generally below those of the pure AlAs and GaAs materials. The LO phonon dispersion curves for GaAs and AlAs do not overlap. Therefore a vibration in one layer can not propagate in the other. Such phonon is localized in its layer. The situation is a little more complicated in $\mathrm{Ga}_{x} \mathrm{Al}_{1-\mathrm{x}} \mathrm{As}$ where there is some overlap which therefore allows some propagation in both layers. 

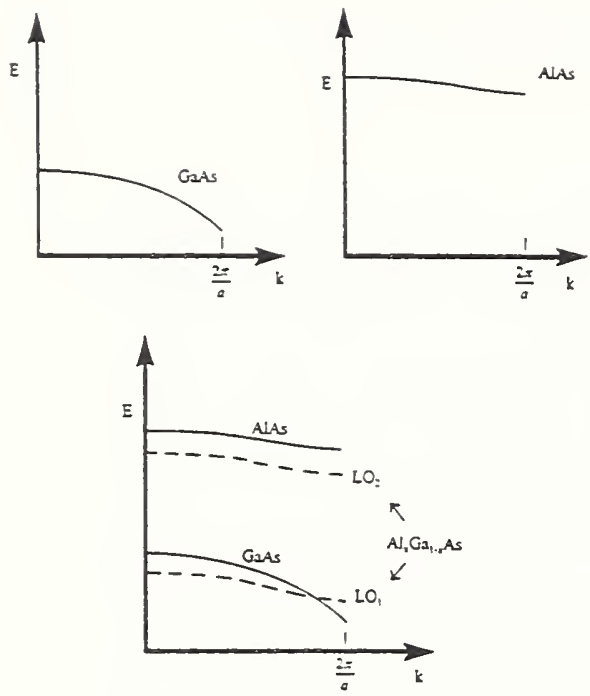

Fig. 1. Dispersion curves for LO phonons.

The confinement of the phonon to one of the layers places it in a quantum well. The model for the system is shown in Fig. 2.

$$
\text { is }
$$
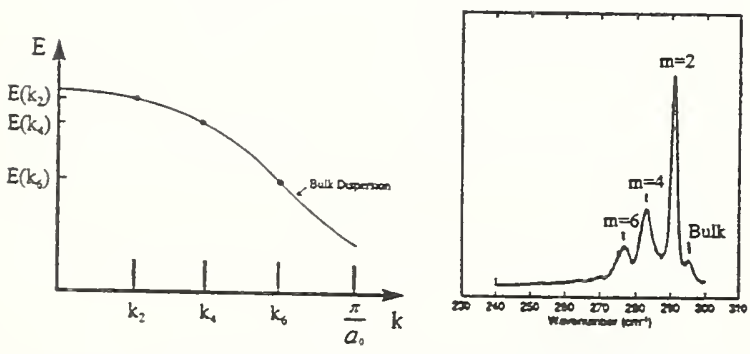

Fig. 2. Model for a GaAs quantum well. Bottom left: Energies for allowed wave vectors for a stack of seven monolayers. Bottom right: Assignment of phonon modes.

The allowed wave vectors $\mathrm{k}$ must obey the relation

$$
\mathrm{k}_{\mathrm{m}}=\mathrm{m}(\omega / \mathrm{L}), \quad \mathrm{m}=0,1,2, \ldots
$$

where

$$
k_{n 1}=\left[n_{1} /(n+1)\right]\left(n / a_{1}\right)
$$

in which

$$
\mathrm{n}=\text { number of monolayers }
$$

and

$$
a_{1}=\text { thickness of one monolayer. }
$$

For the allowed wave vectors $\mathrm{k}_{2}, \mathrm{k}_{4}, \mathrm{k}_{6}$, etc. inside the quantum well one looks up the corresponding energies $E\left(k_{2}\right), E\left(k_{4}\right), E\left(k_{6}\right)$, etc. from the dispersion curve. Only the even modes are allowed in resonant Raman scattering. The Raman spectrum of GaAs/AlAs together with the mode assignment for a stack of seven monolayers each of thickness $0.283 \mathrm{~nm}$ is shown at the bottom right of Fig. 2. These modes of the quantum well are of lower energies and hence downshifted toward lower wavenumbers from that of the bulk $\mathrm{GaAs}$ material.

\section{Raman Scattering by Excitons}

The other system of excitations to be considered is that of excitons. These excitations can be considered in a way similar to that described above for optical phonons. The electron and hole of the exciton are confined to the GaAs layer. Figure 3 shows the energy levels for the electron and the hole and the photoluminescence spectrum of the GaAs/AlAs
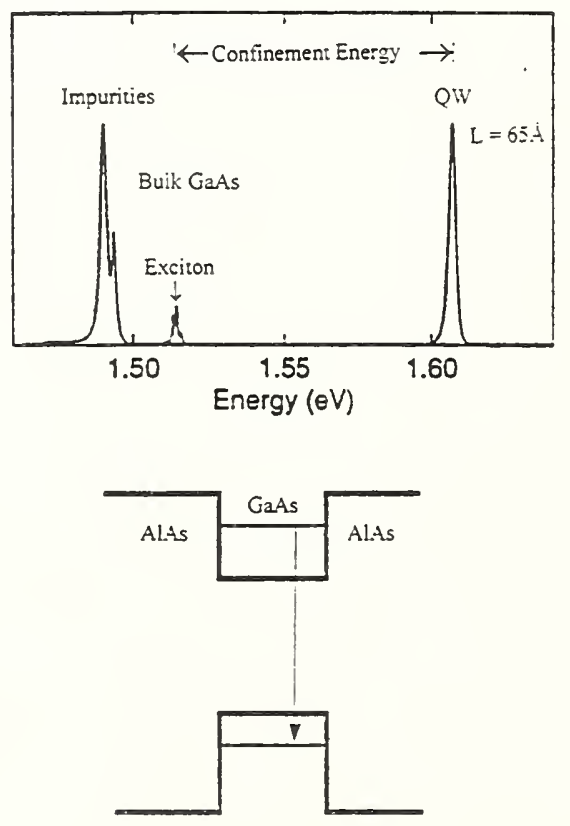

Fig. 3. Photoluminescence spectrum of GaAs/AlAs at $5 \mathrm{~K}$. The layer thickness is $6.5 \mathrm{~nm}$. 
system at a temperature of $5 \mathrm{~K}$. For thickness of the GaAs layer of $6.5 \mathrm{~nm}$ the exciton for the bulk $\mathrm{GaAs}$, at near $1.52 \mathrm{eV}$, is shifted up by the confinement energy of the quantum well to near $1.61 \mathrm{eV}$. This very simple model of phonon and exciton confinement thus gives a reasonably good explanation of the observed spectra. Before going on to the next section I would like to briefly address the topic of resonant Raman scattering. This is actually a very important topic since we are dealing with very, very thin layers, monolayers actually, having thicknesses of a few tenths of nanometers. A monolayer consists of one Ga layer and one As layer. By tuning the laser close to the energy at which the lowest quantum well exciton appears (near 1.60 $\mathrm{eV}$ in Fig. 3) one then finds a huge enhancement in the Raman scattering intensity for the phonons. This is shown in Fig. 4. As the laser energy is tuned from low energy toward and through the exciton (Fig. 3), the phonon, barely discernible as a bump near $1.654 \mathrm{eV}$, increases its intensity, riding on top of the underlying luminescence structure and then decreases again as the laser is tuned beyond the exciton energy. The enhancement in the phonon intensity shown here

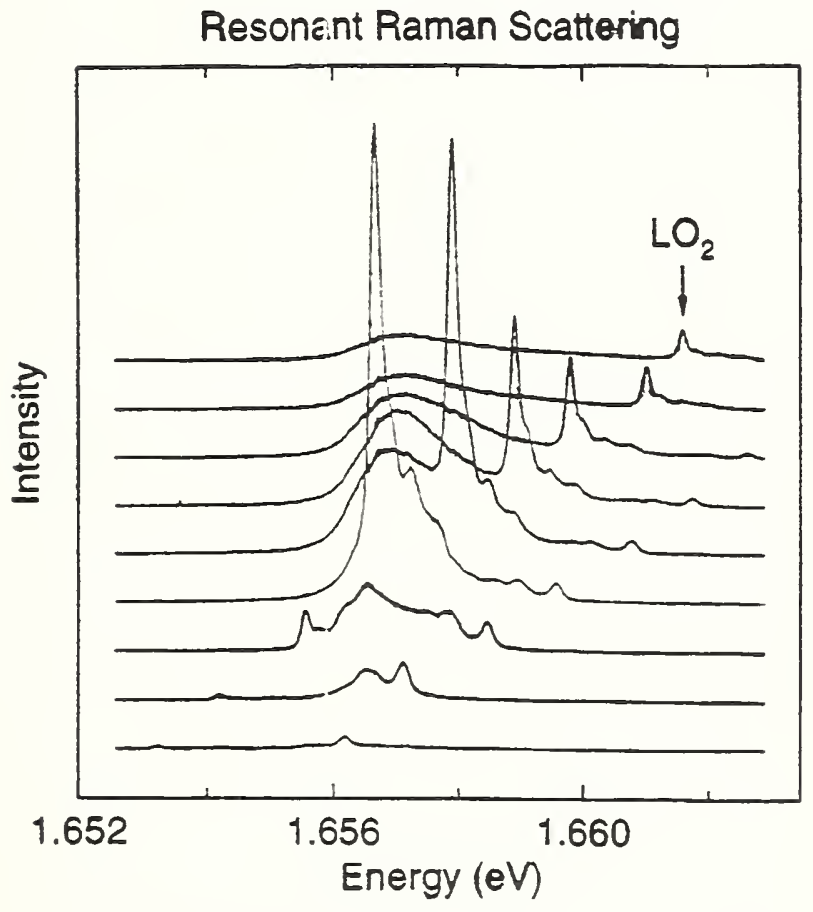

Fig. 4. Resonance Raman scattering by a very thin layer of GaAs/AlAs for different exciting wavelengths. is about three orders of magnitude. (The exciton energy in Fig. 3 is for a layer $6.5 \mathrm{~nm}$ thick, whereas in Fig. 4 the layer is only a few tenths of nanometers thick. This explains the different energies for the exciton resonance in these two figures.) The resonance effect not only enhances the phonon intensities but it also allows us to selectively study the phonons in one or another of the layers. It is thus an important aspect of the Raman scattering technique.

\section{Interface Roughness in GaAs/AlAs Heterostructures}

Perfect interfaces between layers do not exist. There always will be some $\mathrm{Ga}$ or $\mathrm{Al}$ atoms that are in the wrong place, i.e., they are on the wrong side of the interface. The smoothness of interfaces is a subject of intense investigation. Here we limit ourselves to the interface between pure GaAs and AlAs layers [2-7]. In the best samples that have been prepared to date the interface is spread out over 1 to 2 monolayers, the thickness of one monolayer being about 0.28 $\mathrm{nm}$. Not only may the $\mathrm{Ga}$ or $\mathrm{Al}$ atoms be randomly distributed across the interface boundaries but, surprisingly enough, within this layer there exist large, monolayer-high islands.

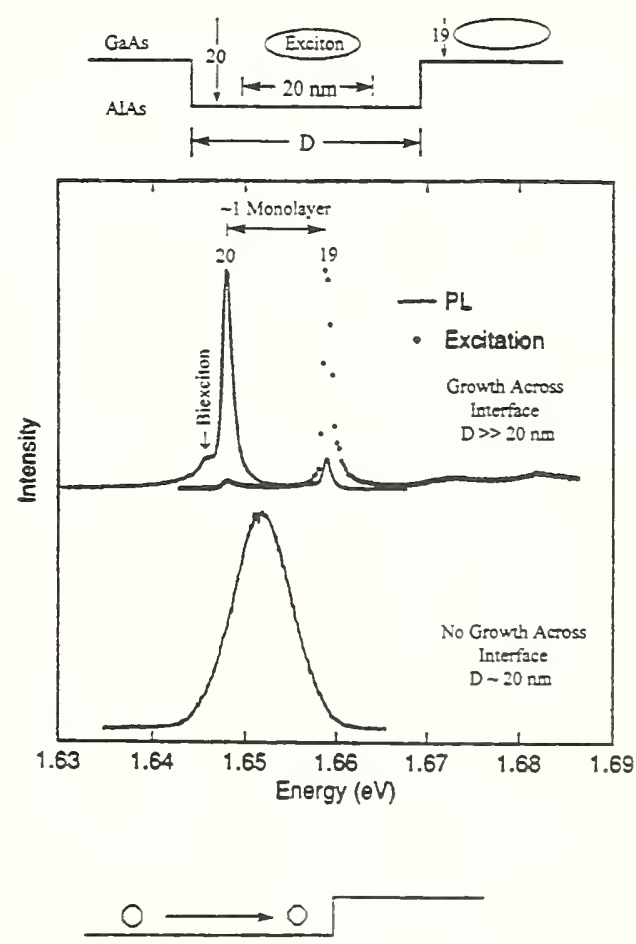

Fig. 5. Photoluminescence spectrum of GaAs/AlAs revealing imperfections across the GaAs-AlAs interface. 
How do we know this and what are the consequences of these irregularities?

The best way to study these effects has turned out to be exciton spectroscopy using oriented samples of GaAs/AlAs such that the probing is conducted along the c-axis of the samples, i.e., perpendicular to the layer stack.

Figure 5 shows such a spectrum. The continuous curve is that of the photoluminescence spectrum while the dotted curve is that obtained via the resonant Raman effect by tuning the laser frequency of the exciting light to that of the exciton, as was described in the previous section. The appearance of the luminescence spectrum depends on the relative size of the exciton $(20 \mathrm{~nm})$ and the lateral extent $D$ of the island.

The bottom spectrum of Fig. 5 is for D $\sim 20$ $\mathrm{nm}$ which is comparable to the size of the exciton. It shows a single exciton peak indicating that the average $\mathrm{D}$ is comparable or less than approximately $20 \mathrm{~nm}$. For a size D much greater than $20 \mathrm{~nm}$ one observes a sharp doublet with splittings that correspond to the difference in the binding energy of one monolayer (top spectrum). This means that the exciton comes either from a region with 19 monolayers or from one with twenty monolayers

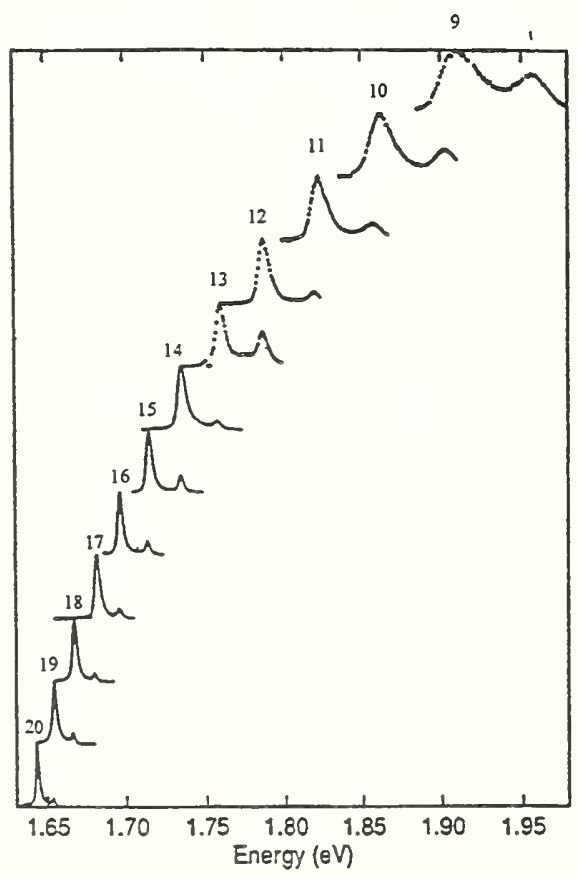

Fig. 6. Photoluminescence spectra for a stack of twenty $\mathrm{GaAs} / \mathrm{AlAs}$ layers. The strong features are those of the excitons while the weak ones are attributed to the phonons. (top of figure). The islands can grow to large diameters and these are the main imperfections across the interfaces of the GaAs and AlAs layers in the best samples of GaAs/AlAs that have been grown so far.

In Fig. 5 only one monolayer is probed and to verify that the observations and their interpretations are correct we probed a stack of specially prepared multilayers [7] by scanning the laser beam across the stack and thus sequentially record the photoluminescence spectra from the different regions as well as the resonance Raman spectra. The results obtained are shown in Figs. 6 and 7 for the luminescence and Raman spectra, respectively.

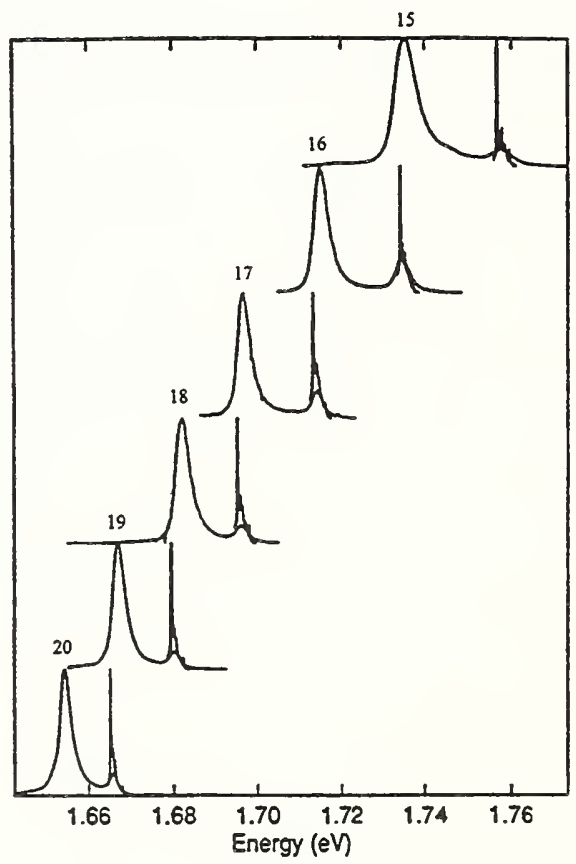

Fig. 7. Resonance Raman spectra of a stack of GaAs/AlAs multilayers. The broad features are those of the excitons while the sharp ones are those of the resonantly enhanced phonons.

The Raman spectra of the different layers exhibit a mode structure due to the confinement of the phonons, as was described in section II, above. This is demonstrated in Fig. 8 for Raman spectra as a function of layer thickness, for monolayers $\mathrm{n}=8$ through $\mathrm{n}=19$. The modes $\mathrm{m}=2$ through $\mathrm{m}=8$ are clearly seen, as is the shift of the mode frequency away from the bulk value toward lower ones. In this figure the largest shift is seen to occur for the $n=8$ monolayer. As the number of monolayers increases the modes move closer to the bulk value. At still lower energies, below 
$270 \mathrm{~cm}^{-1}$, we have the transverse optical modes (TO).

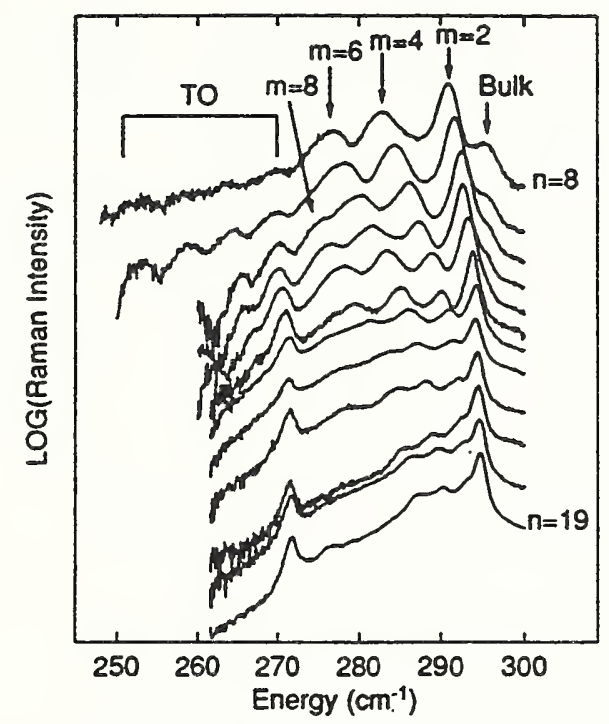

Fig. 8. A logarithmic plot of the resonant Raman spectra of the GaAs phonons for each quantum well width (n). Each of the spectra was recorded in resonance with excitons with energy differences that corresponded to monolayer changes in well width. To the left are the confined LO phonons with $m=2-8$ [4].

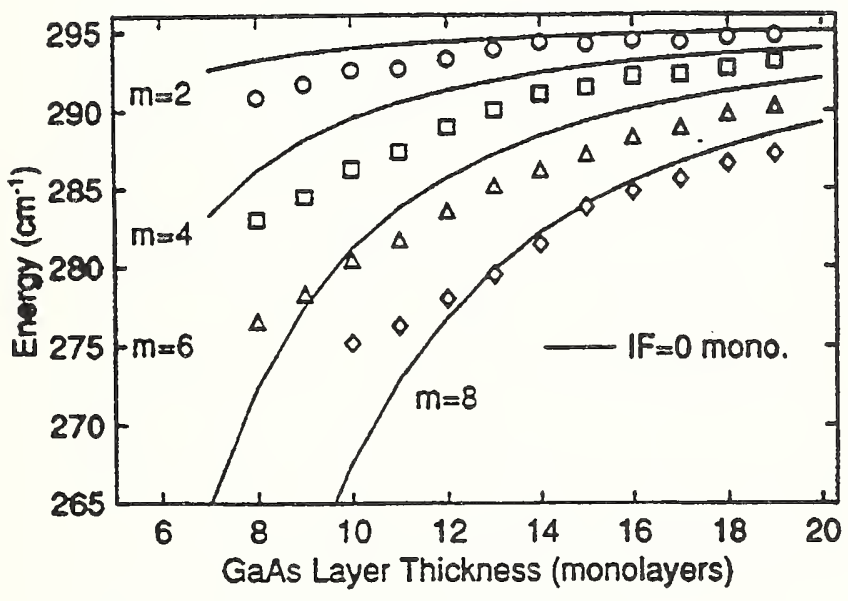

Fig. 9. Comparison of calculated and observed mode energies in the Raman spectra of a GaAs/AlAs stack as function of GaAs layer thickness. The observed data are those shown in Fig. 8 [4].

Figure 9 shows a comparison of the calculated energies for the modes with $\mathrm{m}=2,4,6$, and 8 as function of the GaAs layer thickness with the observed ones. The calculations were carried out for the ideal case of a perfect layer interface and it is seen that the agreement between theory and observation is not very good.
An improvement in the theoretical model is had by allowing not only for gross disorder via monolayer-high islands on the interface, but also by including a fine scale disorder. This is done by considering the linear chain model for each GaAs and AlAs layer and introducing $\mathrm{Al}_{\mathrm{x}} \mathrm{Ga}_{1-\mathrm{x}} \mathrm{As}$ as an alloy at the interface $[8,9]$ (see Fig. 10, top). With this more detailed model there results better agreement between the calculated and observed Raman spectra as is shown in Fig. 10 [5].

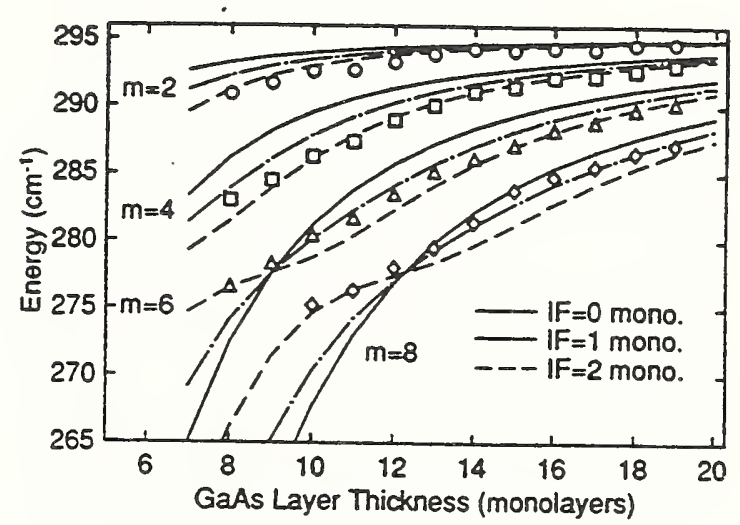

Fig. 10. Observed and calculated mode energies in the Raman spectra of a GaAs/AlAs stack of as function of layer thickness. The calculations allow for fine-scale disorder on the interfaces through inclusion of $\mathrm{Al}_{\mathrm{X}} \mathrm{Ga}_{1-\mathrm{X}} \mathrm{As}$ at the interfaces. The top part of the figure shows the linear chain model of the GaAs/AlAs layer with an alloy of $\mathrm{Al}_{\mathrm{X}} \mathrm{Ga}_{1-\mathrm{X}} \mathrm{As}$ at the interface.

The results of these and other, more inclusive, studies show that even for the very best samples of GaAs/AlAs there are not only large monolayer-high islands at the interfaces, but that there also exists a high density of small scale structure $(<20 \mathrm{~nm})$ within these large islands [5]. This small scale structure is fairly uniform over distances of the order of $0.01 \mu \mathrm{m}$ and its density is dependent on the growth temperature. The small scale structure probably arises from exchanges of the $\mathrm{Ga}$ and $\mathrm{Al}$ atoms as the interface is being formed. This process leads to the formation of a thin $\mathrm{Al}_{\mathrm{x}} \mathrm{Ga}_{1-x}$ As alloy at the interface. Fig. 11 shows several photoluminescence spectra for the $\mathrm{GaAs} / \mathrm{Al}_{0.3} \mathrm{Ga}_{0.7} \mathrm{As}$ system. Specifically, this 
system consists of a $\mathrm{GaAs}$ buffer layer followed by five $\mathrm{GaAs}$ quantum wells with varying widths separated by $25 \mathrm{~nm}$ thick $\mathrm{Al}_{0.3} \mathrm{Ga}_{0.7} \mathrm{As}$ barrier layers. The last barrier layer is followed by a 50 $\mathrm{nm}$ thick GaAs cap (see top diagram of figure) [10]. The lower spectrum is the exciton photoluminescent spectrum and, like the GaAs/AlAs system, shows doublets arising from monolayer-high islands at the interfaces. Its line widths are attributed to inhomogeneous broadening. There is, however, the possibility of resolving this continuous structure into individual lines using the technique of near-field optics.
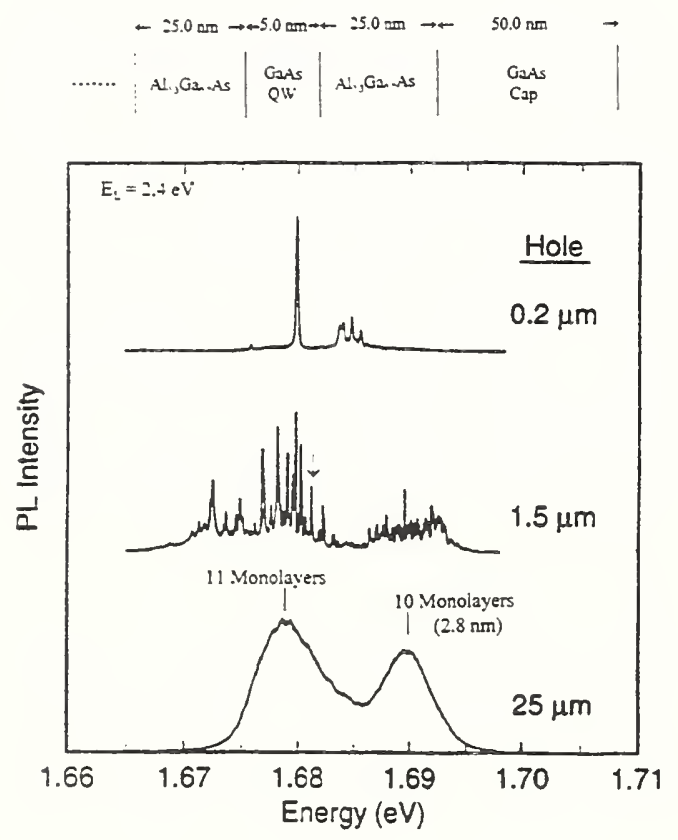

Fig. 11. Photoluminescence spectra of $\mathrm{GaAs} / \mathrm{Al}_{0.3} \mathrm{Ga}_{0.7} \mathrm{As} / \mathrm{AlAs}$ [10].

\section{Optical Near-Field Spectroscopy}

The previous sections dealt with the properties of quantum wells in structures composed of twodimensional layers. In order to also study zerodimensional quantum wells, the so-called quantum dots, it is necessary to do the optical probing at much higher spatial resolutions than is possible with conventionally focused laser beams. Quantum dot structures are formed naturally during the epitaxial growth process, either by potential fluctuations due to monolayer-scale roughness at GaAs quantum well interfaces or by self-organized growth of dots. Both of these growth techniques produce size variations of the dots which results in inhomogeneously broadened photoluminescent lines (see Fig. 11, bottom). However, by probing submicron-size lateral regions of a sample one can resolve the inhomogeneouly broadened spectra into a series of sharp peaks which correspond to the recombination of excitons bound in individual quantum dots.

The essential features of optical near-field imaging and spectroscopy are the following [11]. Instead of focusing the laser beam on the sample to a diffraction limited spot, which represents the conventional far-field optical configuration, the laser beam is sent through an aperture in a metal disk on the sample surface. The aperture can have a diameter less than the wavelength of light. In our work we have used electron-beam lithography to lift off apertures down to $200 \mathrm{~nm}$ diameters in a $100 \mathrm{~nm}$ thick $\mathrm{Al}$ mask evaporated directly on the sample surface [10]. This approach does not allow us to scan the aperture and produce images as in conventional near field scanning optical microscopy using fiber tips [11], but it gives us the opportunity to do optical near field spectroscopy in unprecedented detail.

The bottom spectrum of Fig. 11 was recorded with the near-field optical technique but with an aperture hole diameter of $25 \mu \mathrm{m}$. The spectrum is just as if it were recorded with conventional optical techniques. The photoluminescence arises from the heavy hole exciton and is split into a doublet with a splitting of $11 \mathrm{meV}$. The components of this doublet are due to recombination of the exciton in 10 and 11 monlayer-wide regions of the quantum well The middle and top spectra were obtained with hole diameters of $1.5 \mu \mathrm{m}$ and $0.2 \mu \mathrm{m}$, respectively [10]. The inhomogeneous broad structure of the photoluminescence lines is thus shown to be resolved into many sharp lines which decrease in number with decrease in the aperture hole diameter. These lines reveal the graininess, i.e., the quantum dot nature of the monolayers. These photoluminescence lines arise from excitons recombining in individual quantum dots which are defined by the potential form of the interface fluctuations. One dimension of the dots is determined by the quantum well thickness while the other dimensions are determined by the size of the interface islands $[10,12,13]$.

In the middle spectrum of Fig. 11 a line of a specific quantum dot is singled out with an arrow. The spectrum of this quantum dot is 
shown with higher resolution in Fig. 12. The photoluminescence of the ground state $\left(E_{0}\right)$ of this quantum dot, along with the first six lines

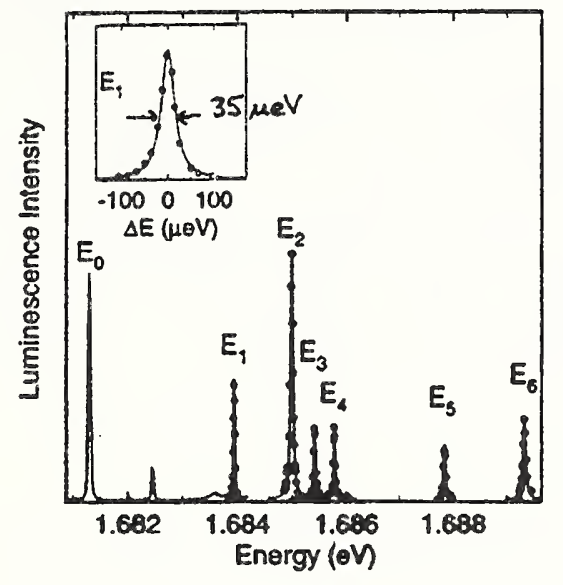

Fig. 12. Photoluminescence spectrum of the quantum dot indicated by the arrow in Fig. 11. $E_{0}$ is the ground state photoluminescence while $\mathrm{E}_{1}-\mathrm{E}_{4}$ are the first six excited states as measured by photoluminescence excitation spectroscopy.

of its excitation spectrum are shown. The way this is done is by detecting the luminescence of one of these spikes $\left(E_{0}\right)$ and then scan the laser, thereby measuring the enhancement in the intensity of this spike due to absorption of the laser radiation. This technique is called photoluminescence excitation spectroscopy. The laser line itself has a width of $\sim 7 \mu \mathrm{eV}(\sim 0.056$ $\mathrm{cm}^{-1}$ ) which thereby determines the limit of resolution of the excitation spectroscopy. The width of the $E_{1}$ line was measured to be $35 \mu \mathrm{eV}$.

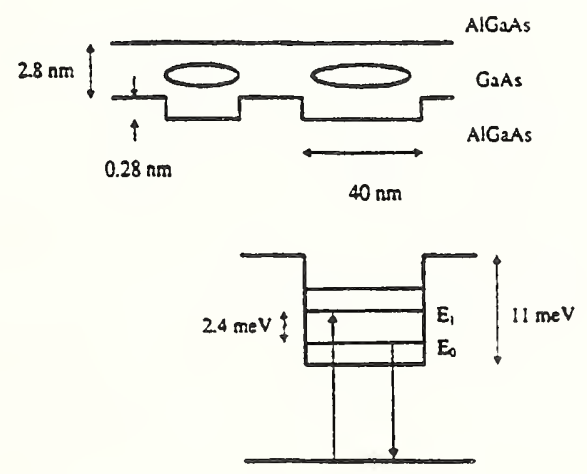

Fig. 13. Potential energies in a quantum dot. The dot is that identified by an arrow in Fig. 11. The diagram is constructed using the data from Fig. 12.

From the excitation spectrum one can estimate the energy levels in the quantum well (see
Fig. 13). There is a localization potential of about $11 \mathrm{meV}$ over a size of $40 \mathrm{~nm}$. This size ranges from about $10 \mathrm{~nm}$ to about $100 \mathrm{~nm}$ from dot to dot. This is just one example, albeit in abbreviated form, that illustrates the technique of single quantum dot spectroscopy.

To summarize the various experiments on single quantum dots performed by us, there is, first, the study of excited state spectroscopy [12], the study of line widths and temperature dependence [13], the investigation of fine structure splittings [13], and also the study of hyperfine interactions of the exciton with nuclear spins [14]. Further work is underway on NMR spectroscopy and magnetic field dependence.

\section{Raman Spectroscopy}

With the strong enhancement available via the resonance Raman scattering one can reduce the laser power and, by tuning the laser through the resonance one can actually sweep the phonon side bands through a quantum dot exciton resonance. This is shown in Fig. 14, where the strong signal is that of the LO phonon and this is where we observe the Raman scattering in a single quantum dot. The aperture used in this experiment was approximately $0.75 \mu \mathrm{m}$. Since there is only one

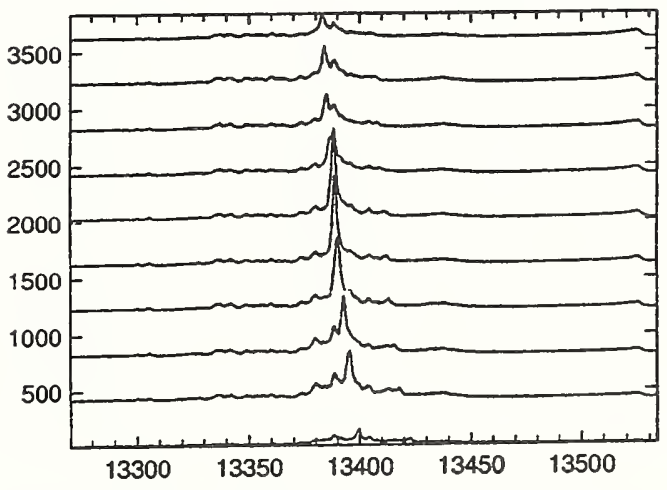

Fig. 14. Resonant Raman scattering and phonon scattering in a single quantum dot.

phonon and one exciton there is no coherent, stimulated amplification; these are spontaneous spectra. We also measured the entire set of optical phonons. In this case the spectrometer was set at the peak and we scanned the (Ti:Sapphire) laser. The resulting spectrum is shown in Fig. 15. 


\section{Summary}

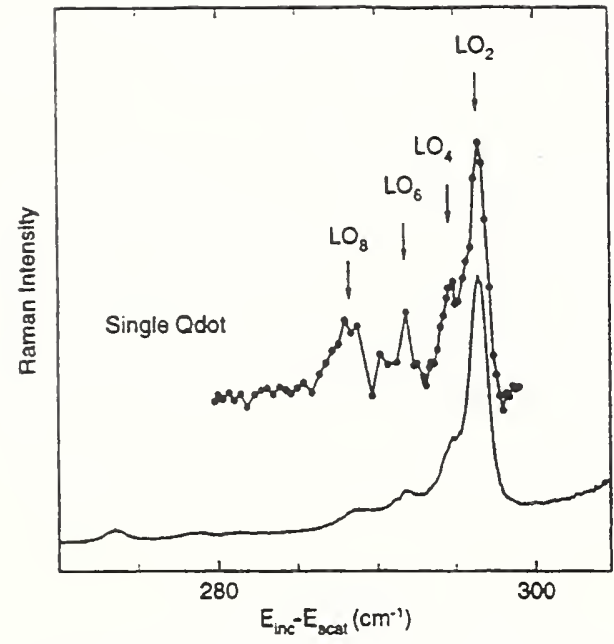

Fig. 15. Raman spectrum of a single quantum dot.

There has been a lot of interest in recent years, certainly since the 1970 s, on micro-Raman scattering. This has now progressed to optical near-field Raman scattering, which, however, is still in the evolution stage and we are very much involved in this. In this method, the lateral size of the sample that is illuminated and imaged is determined by the aperture hole size, which can range down to a diameter of $200 \mathrm{~nm}$. This, then, is the spatial resolution limit that can be achieved with optical near-field Raman spectroscopy. However, the quantum dots are very much smaller in lateral extent. This extent is the size of the quantum dot exciton as seen through the resonance Raman effect. and it is this size that, therefore, defines the spatial resolution that is required. We are actually observing this resolution as demonstrated by the spectrum shown in Fig. 15. This is an order of magnitude better than what one can do with near-field Raman scattering. This domain therefore would be appropriately designated as nano-Raman scattering.

These few examples demonstrate that Raman scattering is a powerful and versatile probe of semiconductor nanostructures. Especially when resonant Raman scattering is used one obtains 1) very high sensitivity so as to allow the recording of a Raman spectrum from a single quantum dot, which encompasses approximately only $10^{5}$ nuclei, and 2) very high selectivity, so that Raman spectroscopy can be performed on a scale of ten nanometers.
Several examples of novel optical techniques have been discussed which allow detailed optical probing of semiconductor nanostructures. These examples have been drawn from our work using photoluminescence-, photoluminescence excitation-, and Raman spectroscopy, the latter done with the emerging techniques of near-field optics. The spatial resolutions that can be reached are unprecedented.

\section{Acknowledgements}

The work described in this paper was conducted at the Naval Research Laboratory. It is a pleasure to acknowledge the contributions made by my collaborators Drs. B.V. Shanabrook, E.S. Snow, D.S. Katzer, and D. Park, as well as the support given by the Naval Research Laboratory. This report was transcribed by Dr. Alfons Weber.

\section{References}

1. See, for example, B. Jusserand and M. Cardona, Raman Spectroscopy of Vibrations in Superlattices, in Light Scattering in Solids $V$, M. Cardona and G. Güntherodt, Eds. (Springer-Verlag, New York, 1989) chapter 3, pp. 49-152.

2. C.A. Warwick, W.Y. Jan, A. Ourmazd, and T.D. Harris, Appl. Phys. Lett. 56, 26662668 (1990).

3. D.S. Katzer, D. Gammon, B.V. Shanabrook, and B. Tadayon, Superlattices and Microstructures 8, 19-24 (1990).

4. D. Gammon, B.V. Shanabrook, and D.S. Katzer, Appl. Phys. Lett. 57, 2710-2712 (1990).

5. D. Gammon, B.V. Shanabrook, and D.S. Katzer, Phys. Rev. Lett. 67, 1547-1550 (1991).

6. D. Gammon, B.V. Shanabrook, and D.S. Katzer, in Proc. SPIE, Spectroscopic Characterization Techniques for Semiconductor Technology IV, O.J. Glembocki, Ed., vol. 1678 (1992) pp.46-55.

7. D. Gammon, B.V. Shanabrook, and D.S. Katzer, Photoluminescence Studies of Interface Roughness in GaAs/AlAs Quantum Well Structures, in Semiconductor Interfaces and Microstructures, Z.C. Feng, 
Ed. (World Scientific Publishing Co., Singapore, 1992) pp. 149-167.

8. J. Leng, Y. Quian, P. Chen, and A. Madhukar, Solid State Comm. 69, 311-315 (1989).

9. B. Samson, S.R.P. Smith, C.T. Foxon, D. Hilton, and K.J. Moore, Solid State Comm. 78, 325-329 (1991).

10. D. Gammon, E.S. Snow, B.V. Shanabrook, D.S. Katzer, and D. Park, Phys. Rev. Lett. 76, 3005-3008 (1996).

11. For a recent account of this subject see, for example, M.A. Paesler and P.J. Moyer, Near-Field Optics: Theory, Instrumentation, and Applications (John Wiley \& Sons, Inc., New York, 1996).

12. D. Gammon, E.S. Snow, and D.S. Katzer, Appl. Phys. Lett. 67, 2391-2393 (1995).

13. D. Gammon, E.S. Snow, B.V. Shanabrook, D.S. Katzer, and D. Park, Science 273, 87$90(1996)$.

14. S.W. Brown, T.A. Kennedy, D. Gammon, and E.S. Snow, Phys. Rev. B 54, R17339R17342 (1996).

\section{Discussion}

Q Regarding the size of the quantum dot, what would be actual spatial resolution? Also, you are actually looking at many quantum dots which are located within the aperture. So what can you say about the localization of the individual dots?

A We must remember that we are really localized, so that we are looking at the atoms in the quantum dot and at individual phonons within that dot. We thus have a local probe, measuring within a $10 \mathrm{~nm}$ square of that sample. We can not tell the location of the dot within the aperture, we can only state that the dot in question is within the area of the aperture, within $200 \mathrm{~nm}$, and that we are measuring the phonons within this dot.

Q You stated that there were large size monolayer-high islands at the interface but you also said that there were island of less than $20 \mathrm{~nm}$ size. How do you know that?

A You do that by photoluminescence excitation spectroscopy. You measure the excited states of the quantum dot. The luminescence gives you the ground state energy of the exciton. The higher energy excitons observed by excitation spectroscopy give you the excited state energies which, in turn, give you the higher modes in the wave function. With this information one then calculates the lateral dimensions of the dot.

Q The resonance occurs when the phonon frequency transfers to the exciton. Isn't this a stimulated Rayleigh proces?

A I do not think that this is stimulated. I think that there is only one photon and one exciton involved in the resonance process.

Q Is there any evidence for the acoustic modes?

A Yes. In superlattices one can study them in detail. In these systems you make use of the folded phonons. I am not sure that you can see acoustic phonons in single quantum wells. 


\title{
Magneto-Raman Spectroscopy at NIST: A Raman Laboratory User Facility
}

\author{
A. Weber, V.B. Podobedov, J.P. Rice, and D.B. Romero \\ Optical Technology Division \\ Physics Laboratory \\ National Institute of Standards and Technology \\ Gaithersburg, MD 20899
}

\begin{abstract}
A new Raman spectroscopy laboratory was set up in early 1995. The laboratory is at present equipped with a modern, state-of-the-art triple spectrometer fitted with a CCD detector. A cryostat equipped with a split-field superconducting solenoid magnet allows the study of Raman spectra of solids over the temperature range from that of liquid helium to $50^{\circ} \mathrm{C}$ and magnetic field strengths from 0 to 8 teslas. The research work focuses on high $T_{C}$ superconducting materials and those that exhibit giant magneto resistance. The facility is available as a user laboratory for the NIST staff and outside investigators. A few initial results obtained with the apparatus are shown.
\end{abstract}

\section{Introduction}

There are at present eleven different research programs at NIST that are based on the technique of Raman spectroscopy. Seven of these are found in the Chemical Sciences and Technology Laboratory, two in the Materials Science and Engineering Laboratory, one in the Electronics and Electrical Engineering Laboratory, and one in the Physics Laboratory. The latter one is the youngest of them, having been started in the spring of 1995. In March 1996 new equipment was installed to allow the development of a competitive program in Raman spectroscopy of materials that are of importance in contemporary science and technology. The research program addresses the phonon and free carrier spectra of materials that are of contemporary scientific and technological importance. Among these are high $T_{C}$ superconducting substances and those that exhibit giant magneto resistance. Other systems are semiconductor heterostructures and thin films.

\section{Experimental Facilities}

A schematic diagram of the experimental apparatus is shown in Fig. 1. The main components are a Dilor XY-800 triple stage spectrometer* equipped with a Spectrum One CCD camera, a Lexel argon ion laser, and a CRYO Industries superconducting magnet housed in a liquid helium cooled cryostat (not shown in the figure). Spectrometer units (1), (2), and (3) are ganged together to form the triple spectrometer. Unit (3) is the main spectrometer part. Usually units (1) and (2) are set for subtractive dispersion and they thus act as a zero dispersion band pass filter. The dispersion and resolution is then defined by unit (3). Units (1) and (2) can also be set to give additive dispersion, thereby increasing the overall dispersion and resolution of the instrument. At the present time only the CCD camera detection system is available.

Table I lists the performance characteristics of the equipment. The Nd:YAG laser source is available but has not yet been integrated into the apparatus.

\footnotetext{
* Certain commercial equipment, instruments, or materials are identified in this paper to adequately specify the experimental procedure. Such identification does not imply recommendation or endorsement by the national Institute of Standards and Technology, nor does it imply that the materials or equipment identified are necessarily the best available for the purpose.
} 


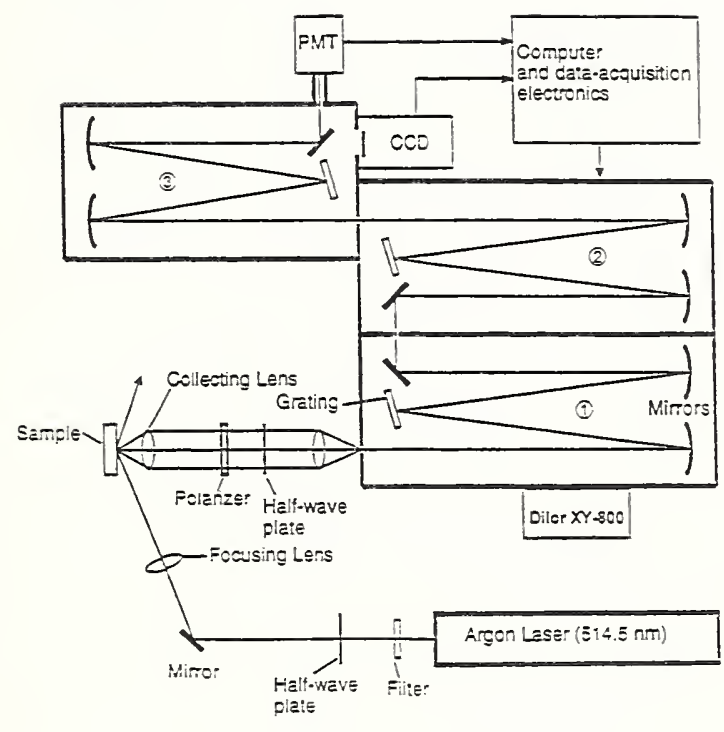

Fig. 1. Shema of a typical Raman experiment. At the present time only the CCD detector is in use. The sample may be illuminated as shown or the laser beam is normally incident on the sample and the scattered light is collected in the "backward scattering" configuration. Experiments are done with the sample located in room air or in a cryostat for temperature and magnetic field studies.

The rotation-vibration spectrum of atmospheric oxygen provides a simple test for the performance of the apparatus. Fig. 2 shows the rotation-vibration Raman spectrum of oxygen gas at atmospheric pressure. The Raman radiation was collected from the focal spot in air of a cw argon laser beam $\left(\lambda_{0}=514.5\right.$ $\mathrm{nm}$ ) with a power of $250 \mathrm{~mW}$. With the spectrometer slits set to a width of $50 \mu \mathrm{m}$ the signal was integrated over a time of $1000 \mathrm{~s}$. The systematically closer spacings of the lines in the

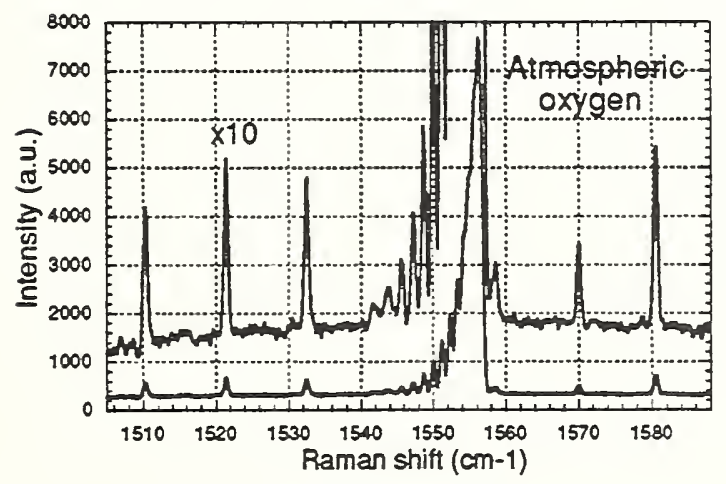

Fig. 2. Rotation-Vibration Raman spectrum of $\mathrm{O}_{2}$. strong $Q$ branch allow an estimate of the resolution while the recording of the weak $\mathrm{O}$ and $S$ branch lines are indicative of the sensitivity. Tests with a neon discharge lamp show that the maximum spectral resolution is close to $0.3 \mathrm{~cm}^{-1}$ when all three spectrometer units are set to operate in the additive mode.

Table I. Specifications of the NIST MagnetoRaman Facility.

\section{Dilor XY 800 Spectrometer with Holographic Gratings and CCD Detector}

$\begin{array}{ll}\text { Focal length: } & \mathrm{f}=800 \mathrm{~mm} \\ \text { Relative aperture: } & \mathrm{f} / \mathrm{d}=14\end{array}$

Multichannel detection: $\sim 650 \mathrm{~cm}^{-1}$ per frame in the range from $450 \mathrm{~nm}$ to $750 \mathrm{~nm}$ (Raman shift from $\sim 5 \mathrm{~cm}^{-1}$ to $\sim 10^{4} \mathrm{~cm}^{-1}$ )

Maximum resolution: $\sim 0.3 \mathrm{~cm}^{-1}$ (at $\sim 514.5 \mathrm{~nm}$ )

$\lambda / 2$ and $\lambda / 4$ retardation plates

Polarization analyzer

Software for treating spectra

Optical Cryostat with Superconducting Magnet System

Temperature range: $\quad$ from $2 \mathrm{~K}$ to $323 \mathrm{~K}$ Magnetic field: up to $8 \mathrm{~T}$

Maximum relative aperture: $f / d=3$

$$
\text { cw Argon Laser }
$$

$\lambda=514.5$ or $488 \mathrm{~nm}$, with powers up to $\sim 2 \mathrm{~W}$ About 10 additional lines with powers from 10 to $50 \mathrm{~mW}$

\section{Nd:YAG Laser}

\section{$\underline{1064 \mathrm{~nm}} \underline{532 \mathrm{~nm}}$}
a) $\mathrm{cw}$
$12 \mathrm{~W} \quad \mathrm{~N} / \mathrm{A}$
b) Q-switched (150 ns)
$14 \mathrm{~kW} \quad \mathrm{~N} / \mathrm{A}$
c) Mode-locked (<150 ps)
$1 \mathrm{~kW} 500 \mathrm{~W}$
d) $b$ and $c$
$800 \mathrm{~kW} \quad 500 \mathrm{~kW}$

\section{Yttrium Barium Cuprate}

Yttrium Barium Cuprate, $\mathrm{YBa}_{2} \mathrm{Cu}_{3} \mathrm{O}_{7-\delta}$, (YBCO) is material currently under intensive 
investigation because it is a superconductor up to the relatively high critical transition temperature $\mathrm{T}_{\mathrm{C}}=92 \mathrm{~K}$.

One of the central postulates of the BardeenCooper-Schrieffer (BCS) microscopic theory of superconductivity is the pairing mechanism. The attractive interaction of electrons mediated by electron-phonon coupling is the mechanism for Cooper pair creation in the classical superconductors [1]. After the discovery of copper oxide high- $T_{C}$ superconductors the importance of the phonon mechanism was widely discussed in the literature. Light scattering experiments have played an important role in these discussions, the particular focus being on the characteristic energy gap and its anisotropy. Measurements of the $340 \mathrm{~cm}^{-1} \mathrm{~B}_{1 \mathrm{~g}}$ Raman phonon mode in $\mathrm{RBa}_{2} \mathrm{Cu}_{3} \mathrm{O}_{7-\delta}$ compounds $(\mathrm{R}=\mathrm{Y}, \mathrm{Eu}, \mathrm{Dy}, \mathrm{Er}, \mathrm{Tm})$ have clearly shown the effect of superconductivity on the phonon spectrum [2] and made it possible to give an estimate of the value of the electronphonon coupling constant $\lambda$ to be in the range from $\sim 0.5$ to 1.0 . There is, however, a belief that this value may be insufficient to explain superconductivity in $\mathrm{RBa}_{2} \mathrm{Cu}_{3} \mathrm{O}_{7-\delta}$ compounds [3] so that there is still no agreement on the definite mechanism of the charge-carrier pairing responsible for superconductivity.

Although the magnetic field is known to be an important variable affecting superconductivity, there have been only two magneto-Raman studies of high- $T_{C}$ superconductors so far $[4,5]$. Besides its use in the study of the crystal lattice phonons, the magneto-Raman effect has also been suggested as a probe of high- $T_{C}$ superconductors in order to improve the understanding of the role of electronic Raman scattering [6]. One of the important questions is whether the continuum in Raman scattering from high- $T_{C}$ superconductors involves electronphonon coupling. It is against this background that we undertook a study of the temperature and magnetic field dependence of the Raman spectrum of YBCO.

The unit cell structure of the YBCO crystal is shown in Fig. 3. The anti-symmetric vibrations of the OII and OIII atoms in the unit cell give rise to the Raman $B_{1 \mathrm{~g}}$ mode at $340 \mathrm{~cm}^{-1}$, which is the mode characteristic of the Raman spectrum of YBCO. The Raman spectrum recorded in 20 minutes from a single crystal of this material at $\mathrm{T}=35 \mathrm{~K}$ and at room temperature in the region from $40 \mathrm{~cm}^{-1}$ to $660 \mathrm{~cm}^{-1}$ is shown in Fig. 4. The two spectra are aligned with

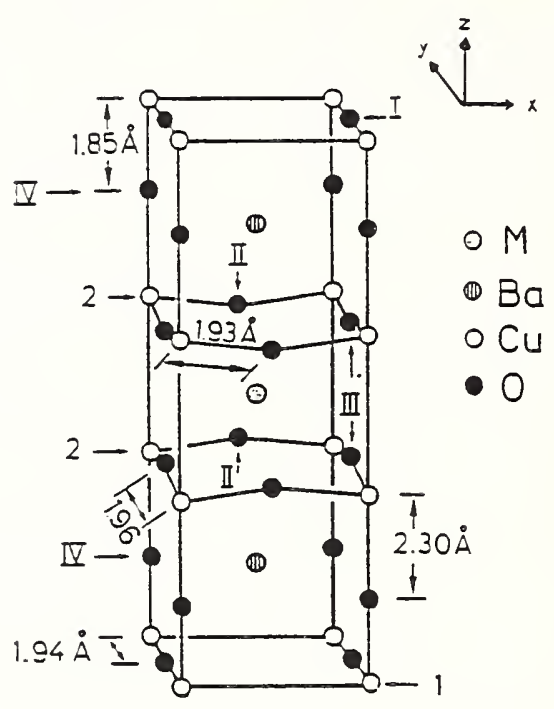

Fig. 3. Structure of the unit cell of $\mathrm{YBa}_{2} \mathrm{Cu}_{3} \mathrm{O}_{7}$.

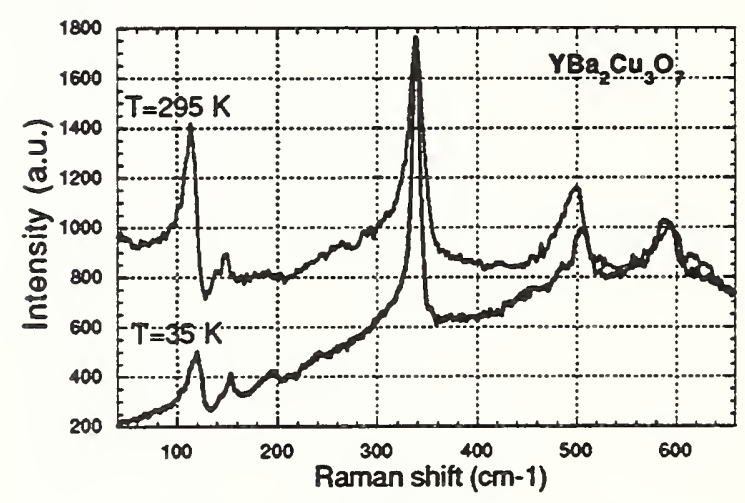

Fig. 4. The Raman spectrum of YBCO.

respect to the $340 \mathrm{~cm}^{-1}$ line and are off set for clarity. We see the usual shift in the phonon modes with temperature as well as the significant narrowing of the $340 \mathrm{~cm}^{-1}$ line with cooling. Whereas the continuum at room temperature rides on a fairly flat base line, it has a remarkable positive slope at $35 \mathrm{~K}$.

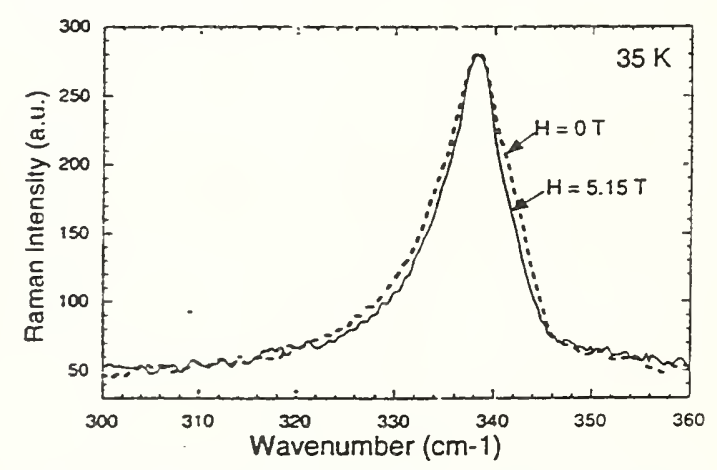

Fig. 5. Magnetic field dependence of the line width of the $\mathrm{B}_{1 \mathrm{~g}}$ mode in $\mathrm{YBCO}$ at $35 \mathrm{~K}$. 
The effect of the magnetic field on the width of the $340 \mathrm{~cm}^{-1}$ line at $35 \mathrm{~K}$ is shown in Fig. 5 , and the overall temperature dependence of this width for zero field and for $\mathrm{H}=5.15 \mathrm{~T}$ is shown in Fig. 6 [7]. Below the transition temperature the line width is fairly constant but is narrower by about $20 \%$ compared to the value at zero field. For the two field conditions the line width increases as the temperature approaches the critical value, but with slightly different slopes.

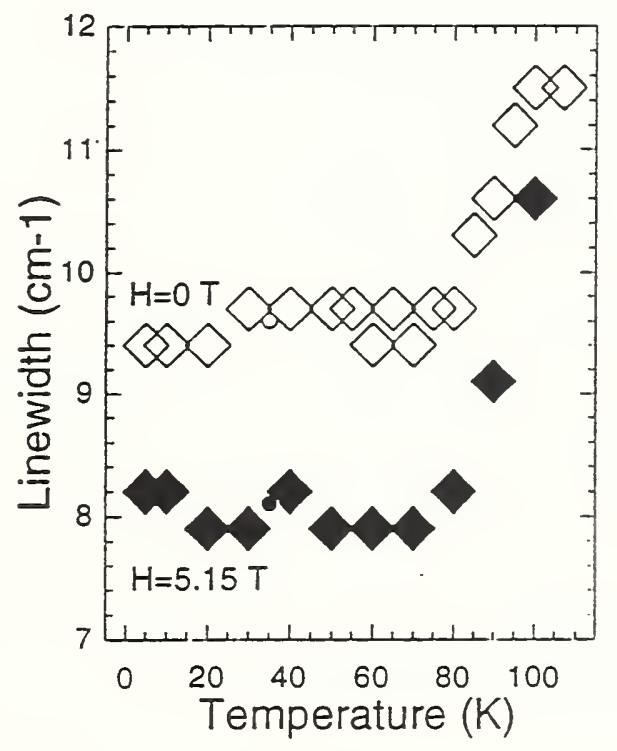

Fig. 6. Temperature dependence of the line width of the $340 \mathrm{~cm}^{-1}$ Raman mode in a YBCO single crystal.

The recording time was 20 minutes per point.

The continuous background at low temperature (see Fig. 4) also changes with magnetic field intensity. Here the measurements of the continuum intensity were made at a Stokes

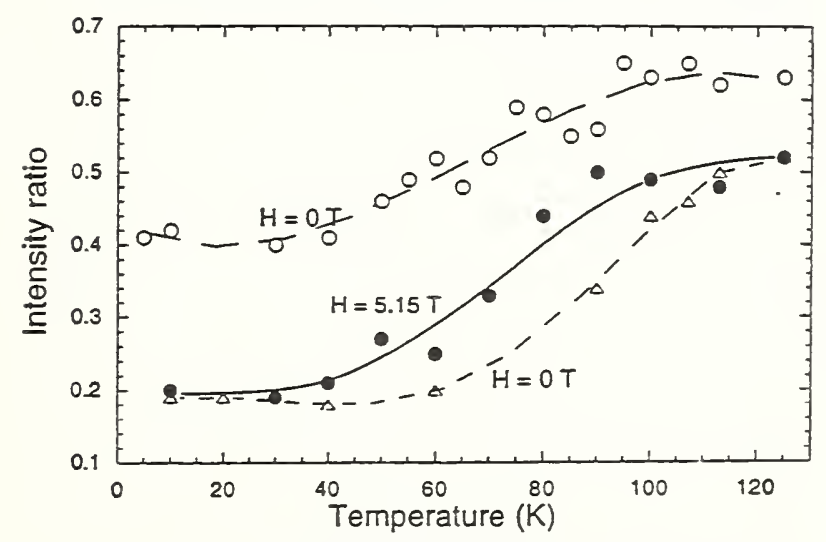

Fig. 7. Magneto-temperature dependence of the Raman continuum in $\mathrm{YBCO}$ at $40 \mathrm{~cm}^{-1}$.
Raman shift of $40 \mathrm{~cm}^{-1}$. These were normalized to the peak integrated intensity of the $340 \mathrm{~cm}^{-1}$ line which thus served as an internal standard. Figure 7 shows this behavior as function of temperature for zero field and for $\mathrm{H}=5.15 \mathrm{~T}$. All three curves exhibit the same trend. The two lower curves depict the behavior of the same sample spot. At low and high temperatures the curves for $\mathrm{H}=0 \mathrm{~T}$ and for $\mathrm{H}=5.15 \mathrm{~T}$ merge into one another. Their difference between $c a$. $30 \mathrm{~K}$ and $120 \mathrm{~K}$ is attributed to the nature of the continuum which is mainly due to electronic excitations arising from the pair breaking process. The top $\mathrm{H}=0$ curve derives from a different spot on the sample. This shows that the crystal sample was not uniform.

\section{Lanthanum Manganate}

The following material shows preliminary results obtained for the polarized Raman spectra of undoped and doped $\mathrm{LaMnO}_{3}$ crystals. The lanthanum manganates are representative of a class of crystals that may, under suitable conditions, exhibit a giant magneto resistance effect, and the investigations of their properties, just as those for the high- $T_{C}$ superconductors, are of technological importance.

Figure 8 shows the Raman spectrum of lanthanum manganate at different temperatures with the strong line at $c a .610 \mathrm{~cm}^{-1}$ being the feature of interest. There is the usual line narrowing as the temperature is lowered, accompanied by a small shift toward lower wave numbers. Below $140 \mathrm{~K}$, however, this shift becomes abrupt for the $120 \mathrm{~K}$ signature, at a rate that differs from that at which the shifts occur at

\section{P - CA phase transition}

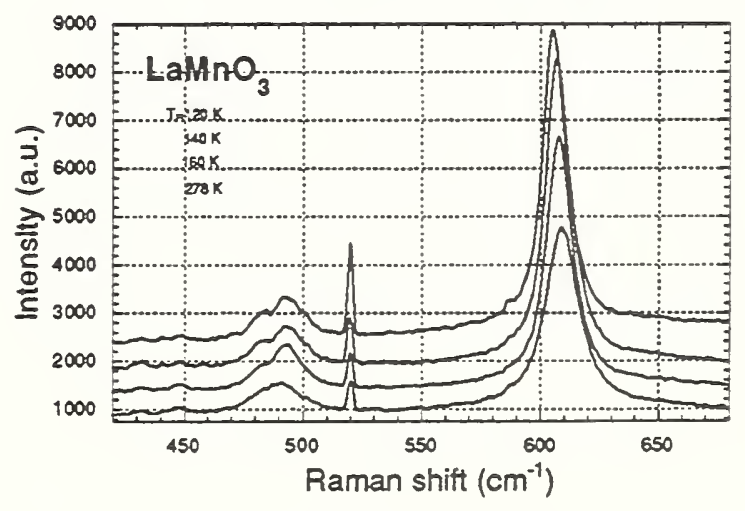

Fig. 8. Raman spectrum of $\mathrm{LaMnO}_{3}$ at different temperatures. 
the higher temperatures. This abrupt change from $140 \mathrm{~K}$ to $120 \mathrm{~K}$ correlates with the well known transition of this material from the paramagnetic to the canted anti-ferromagnetic phase. These line shifts are real and are not due to instrumental effects. The evidence for this is the unchanged position of the line at $520 \mathrm{~cm}^{-1}$, which is not a Raman line at all, but is a line from the argon laser plasma that is also (Rayleigh) scattered by the crystal.

Lanthanum manganate, $\mathrm{LaMnO}_{3}$, is an ordinary material which does not exhibit a giant magneto resistance effect. An attempt was therefore made to look at the Raman spectrum of strontium-doped lanthanum manganate, as the doped version is known to show this effect. Figure 9 shows the yy- and xy-polarized Raman spectra of undoped $\mathrm{LaMnO}_{3}$, curves (1) and (2) respectively, while the spectrum of the strontium-doped species, $\mathrm{La}_{0.7} \mathrm{Sr}_{0.3} \mathrm{MnO}_{3}$, is shown at a magnification of $12 \mathrm{x}$ in curve (3). The strong Raman features seen for $\mathrm{LaMnO}_{3}$ do not show in curve (3), suggesting that upon doping the material underwent a transition to a cubic phase in which the $A_{g}$ and $B_{1 g}$ modes are not allowed in first order Raman scattering. The weak Raman features for the doped material suggest that they are due to second order Raman scattering $[8,9]$. (The sharp lines in all three spectra are those due to the argon laser plasma.)

\section{Modes assignment $(T=300 \mathrm{~K})$}

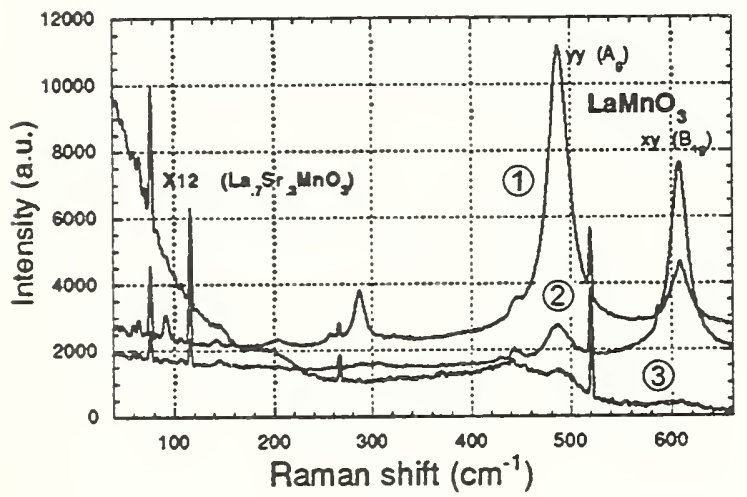

Fig. 9. Raman spectra of doped and undoped lanthanum manganate at room temperature.

\section{A Raman User Laboratory}

Though there are many groups at NIST already engaged in Raman spectroscopy, there are often needs for Raman spectra expressed by those who do not have their own equipment. Also, in other cases the available equipment may be found to be inadequate for specific requirements. The present laboratory is therefore planned to also serve as a user facility for the NIST staff and outside investigators. The following example illustrates this function.

Raman spectroscopic studies of dental materials are pursued in the Polymers Division of the Materials Science and Engineering Laboratory. One of these materials is the dental ceramic apatite, $\mathrm{Ca}_{10}\left(\mathrm{PO}_{4}\right)_{6}(\mathrm{OH})_{2}$, for which the room temperature Raman spectrum provided by

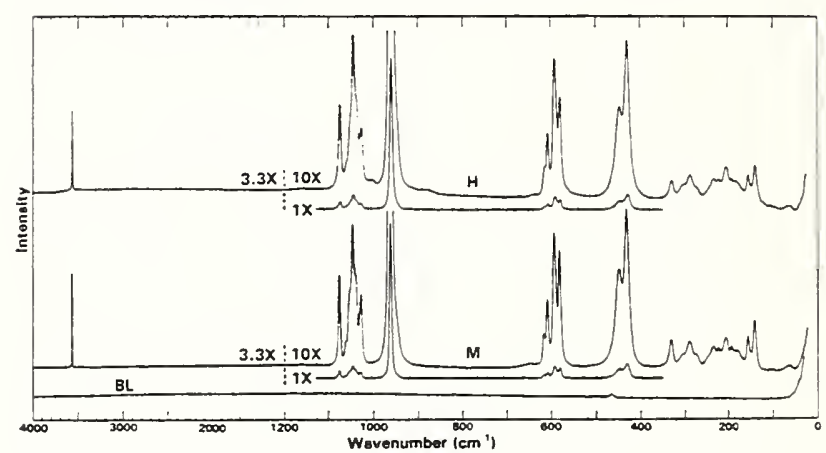

Fig. 10. Room temperature Raman spectra of apatite at a resolution of $3 \mathrm{~cm}^{-1} . \mathrm{H}=$ hexagonal phase, $\mathrm{M}=$ monoclinic phase, $\mathrm{BL}=$ background level. (Spectrum provided by B. Fowler.)

Mr. B. Fowler is shown in Fig. 10. Not all of the expected Raman active vibrations were observed, however, even with the material cooled to liquid nitrogen temperature. Most of these missing Raman lines were suspected to be found in the crowded low frequency region, up to about $600 \mathrm{~cm}^{-1}$, as well as the region around $1040 \mathrm{~cm}^{-1}$. We therefore recorded the Raman spectrum of apatite at very low temperatures and with higher spectral resolution.

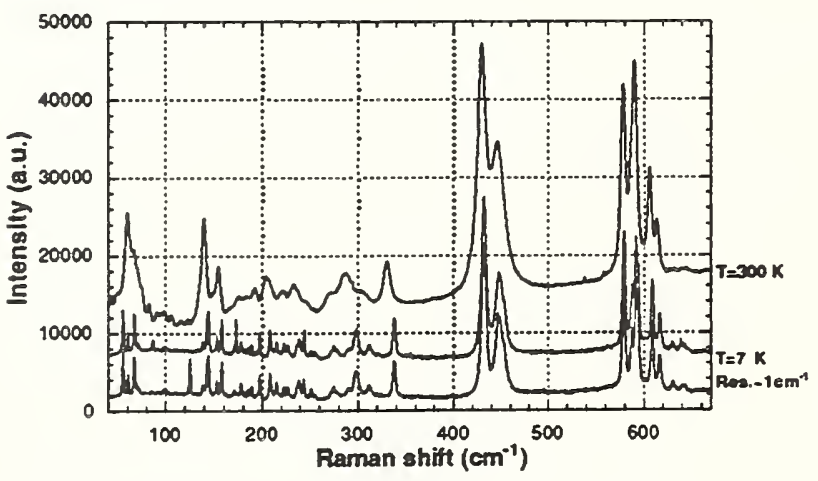

Fig. 11. Raman spectra of apatite at $1 \mathrm{~cm}^{-1}$ resolution. 
Figures 11 to 13 show the spectra recorded with our instrument. The very low temperature spectra, at $\mathrm{T}=7 \mathrm{~K}$, now reveal many resolved features even at a resolution of only $1 \mathrm{~cm}^{-1}$ (Figs. $11,12)$ The region about the $1040 \mathrm{~cm}^{-1}$ line measured at a resolution of $0.4 \mathrm{~cm}^{-1}$ and a temperature of $7 \mathrm{~K}$ reveals 15 out of a total of 18

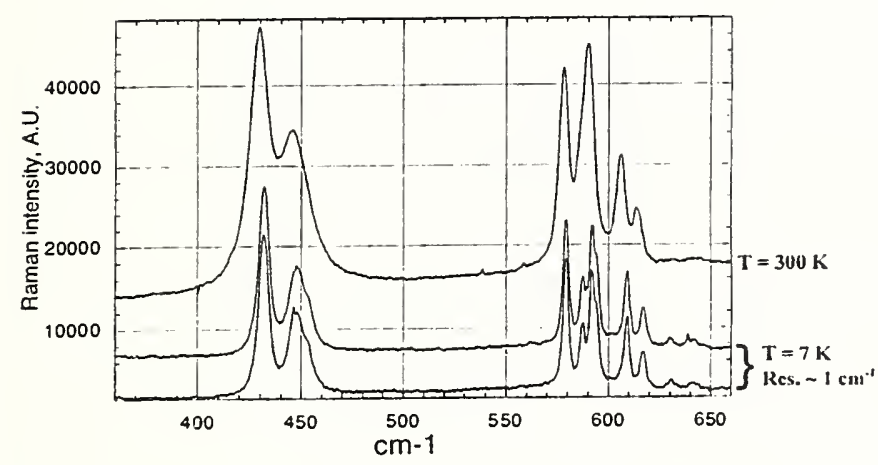

Fig. 12. Raman spectra of apatite at $1 \mathrm{~cm}^{-1}$ resolution. The region from $350 \mathrm{~cm}^{-1}$ to $650 \mathrm{~cm}^{-1}$.

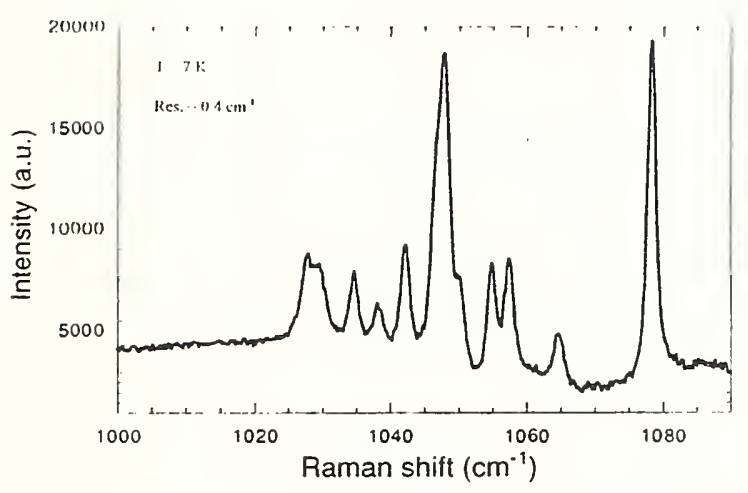

Fig. 13. Raman spectrum of apatite at $0.4 \mathrm{~cm}^{-1}$ resolution and at $T=7 \mathrm{~K}$.

expected Raman frequencies. Previously only 12 lines were observed in this region (Fig. 13). Two low temperature spectra are shown in Fig. 11 as a check on the reproducilibity of the results. Close study reveals that there are sharp spikes in these spectra, near $95 \mathrm{~cm}^{-1}, 125 \mathrm{~cm}^{-1}$, $160 \mathrm{~cm}^{-1}$, and other locations which are not common to both spectra. These are not laser plasma lines but are random noise spikes originating in the CCD detector. These become more prominent for longer signal integration times and care must be taken to avoid misinterpretation of such features.

\section{Summary}

A new program in Raman spectroscopy was started in 1995 with the aim of investigating novel materials of importance to contemporary science and technology, and to also provide a resource to NIST staff and other investigators in need of Raman spectroscopic data. Equipped with new apparatus in the spring of 1996, the laboratory is now productive in the areas of high- $T_{C}$ superconducting materials, materials that exhibit giant magneto resistive effects, semiconductors, and thin films. Several current research results as well as an application of the user laboratory assisting others have been described in this report.

\section{References}

1. J. Bardeen, Science 181, 1209 (1973).

2. B. Friedl, C. Thomsen, and M. Cardona, Phys. Rev. Lett. 65, 915 (1990) and references cited therein.

3. C. Thomsen, J. Raman Spectrosc. 27, 329 (1996) and references cited therein.

4. T. Ruf, C. Thomsen, R. Liu, and M. Cardona, Phys. Rev. B 38, 11985 (1988).

5. T. Ruf, E.T. Heyen, M. Cardona, J. Mescot, and A. Furre, Phys. Rev. B 46, 11792 (1992).

6. E.G. Mishchenko, Phys. Rev. B 53, 2083 (1996).

7. V.B. Podobedov, J.P. Rice, A. Weber, and H.D. Drew, J. Supercond. 10,205-209 (1997).

8. V.B. Podobedov, A. Weber, J.P. Rice, D.B. Romero, and H.D. Drew, Bull. Am. Phys. Soc. 42, 341 (1997).

9. V.B. Podobedov, A. Weber, J.P. Rice, D.B. Romero, and H.D. Drew, (to be published). 


\section{List of Speakers}

Dr. Michael I. Bell, Naval Research Laboratory

Dr. Bell received his Ph.D. degree in solid state physics from Brown University in 1972. $\mathrm{He}$ was a visiting scientist at the Max-Planck-Institut für Festkörperforschung in Stuttgart, Germany during 1972-1973 after which he joined the faculty of Yeshiva University in New York as Associate Professor of Physics and Fellow of the Maybaum Institute for Quantum Electronics and Materials Science. In 1978 he joined NIST (then known as the National Bureau of Standards, NBS) where he conducted research on the optical and electronic properties of semiconductors, ceramics, and polymers. In $1990 \mathrm{Dr}$. Bell joined the Naval Research Laboratory where he is now Head of the Dynamics of Solids Branch of the Condensed Matter and Radiation Sciences Division. His principal research interests are the optical spectroscopy of solids, including Raman spectroscopy and $\mathrm{x}$-ray absorption measurements using synchrotron radiation. He has served as a consultant to private industry and to several government agencies, including the Department of Defense, the Food and Drug Administration, and the Federal Reserve Board. He is a member of the American Physical Society, The American Association for the Advancement of Science, The American Society for Testing and Materials, and Sigma Xi.

Dr. Edgar S. Etz, National Institute of Standards and Technology

Dr. Etz received his Ph.D. degree in 1967 from Clarkson University whereupon he joined NIST (then known as the National Bureau of Standards, NBS) to do research in electrochemistry and solution thermodynamics in the Analytical Chemistry Division of the Chemical Sciences and Technology Laboratory. In 1974 he began the development of a Microprobe Raman spectrometer. Since that time he has been engaged in Raman spectroscopy with emphasis on instrumentation and techniques development. $\mathrm{He}$ is also actively conducts Fourier-transform infrared microspectroscopy as an adjunct to other microprobe techniques.

Dr. Bruno M. Fanconi, National Institute of Standards and Technology

Dr. Fanconi received his Ph.D. degree in Physical Chemistry from the University of Washington, in Seattle, Washington. He joined NIST in 1971 and is the leader of the Polymer Characterization Group in the Materials Science and Engineering Laboratory.

Dr. Daniel G. Gammon, Naval Research Laboratory

Dr. Gammon received his Ph.D. degree in Physics from the University of Michigan in 1987 for research on Raman scattering done under the direction of Prof. Roberto Merlin. In 1987 he joined the Naval Research Laboratory as a National Research Council, National Academy of Sciences Postdoctoral Research Associate and he has remained there as a regular staff member. His research is in the area of Raman spectroscopy of heterostructures. He is also engaged in non-liner optics after having been on a sabbatical appointment at the University of Michigan during the past year. 
Dr. Christopher A. Kendziora, Naval Research Laboratory

Dr. Kendziora is a member of the Directed Energy Effects Branch at the Naval Research Laboratory after having been a National Research Council, National Academy of Sciences Postdoctoral Research Associate in that institution. The research for his doctorate was on the crystal growth and characterization of high temperature superconductors and was conducted at the State University of New York at Stony Brook under the direction of Prof. Laszlo Mihaly. His recent research was on the scattering properties of several high- $\mathrm{T}_{\mathrm{C}}$ superconductors as a function of carrier concentration and superconducting transition temperature.

\section{Dr. M. Anne Leugers, Dow Chemical Corporation}

Dr. Leugers is a Research Leader in the Spectroscopy Group of the Analytical Sciences Laboratory at the Dow Chemical Company. She received her B.S. in Chemistry from Xavier University and her Ph.D. in Physical Chemistry from the University of Cincinnati in 1981. Dr. Leugers performed postdoctoral research at Syracuse University and at the University of Arizona before taking a research position in the paper industry studying the materials science of paper. She joined the Dow Chemical Company in 1984, where she began working in the area of fiber-optic and Raman spectroscopy. Dr. Leugers's research interests include application of Raman and fiber-optic spectroscopy to the chemical and morphological characterization of synthetic polymers. She is the holder of two U.S. patents, and has filed several other patent disclosures. She has also authored eight publications in the area of high-resolution, laser spectroscopy, and IR spectroscopic reviews.

\section{Dr. John F. Rabolt, University of Delaware}

Dr. Rabolt is Professor and Chairman of the Materials Science Department at the University of Delaware. After receiving his B.S. from the State University of New York College at Oneonta in 1970 he engaged in graduate studies and research at Southern Illinois University from which he received his Ph.D. in Physics in 1974. In 1974-1975 he was a postdoctoral fellow in Physics at the University of Michigan and a National Research Council, National Academy of Sciences Research Associate at the National Bureau of Standards in 1976-1977. In 1978 he joined the IBM Almadén Research Laboratories where he was a research staff member of the Polymer Physics and Chemical Physics Group. His recent research was devoted to the use of Fourier transform infrared, and Fourier transform- as well as conventional Raman spectroscopy to investigate the crystal and molecular structures of long chain molecules and polymers. He developed methods for the integration of optical techniques in conjunction with Raman spectroscopy to investigate sub-micron polymer films and polymer surfaces. He also performed Fourier transform infrared spectroscopic investigations of self-assembled and Langmuir-Blodgett films on metals and dielectrics. He is a co-developer of the technique of Fourier transform Raman spectroscopy with near infrared excitation and its application to vibrational spectroscopy. $\mathrm{He}$ is co-editor of the book Fourier Transform Raman Spectroscopy: From Concept to Practice. In 1996 he joined the faculty of the University of Delaware as Professor and Chairman of the Department of Materials Science. 
Dr. Alfons Weber, National Institute of Standards and Technology

Dr. Weber is Senior Scientist in the Physics Laboratory of NIST (formerly the National Bureau of Standards, NBS) where he conducts research in Raman spectroscopy of novel solid state materials in the Optical Properties and Infrared Technology Group of the Optical Technology Division. Dr. Weber did his undergraduate and graduate studies at the Illinois Institute of Technology, from which he received the Ph.D. degree in Physics in 1956 for research in infrared and Raman spectroscopy under the direction of Prof. F.F. Cleveland. In 1956-1957 he was a National Research Council of Canada postdoctoral fellow at the University of Toronto where he engaged in high resolution Raman spectroscopy of gases in the group headed by H.L. Welsh. In 1957 he joined the Physics Department of Fordham University in New York where he established a research program in high resolution Raman spectroscopy of gases. In 1977 he joined the National Institute of Standards and Technology (NIST) (then known as the National Bureau of Standards, NBS) where he conducted research in high resolution infrared spectroscopy. After assignments to the Chemical Sciences Division in the Office of Basic Energy Sciences of the Department of Energy, and the Division of Chemical Sciences of the National Science Foundation, he is now Senior Scientist in the Physics Laboratory of NIST. There he conducts research on the Raman spectra of high- $T_{C}$ superconducting materials, materials that exhibit giant magneto resistive effects, and other materials of current scientific and technological importance. Among his numerous publications are review articles on high resolution Raman spectroscopy and several edited books on Raman spectroscopy and molecular spectroscopy in general. He was the first to successfully demonstrate the feasibility of doing Raman spectroscopy with a Fourier transform spectrometer. He was on the editorial boards of the Journal of Raman Spectroscopy and the Journal of Physical and Chemical Reference Data. He is a Fellow of the American Physical Society and a member of the Optical Society of America, the Coblentz Society, the Society for Applied Spectroscopy, and the American Association for the Advancement of Science. 


\section{List of Attendees}

Andreas C. Albrecht

Baker Laboratory of Chemistry

Cornell University

Ithaca, NY 14850

Tel: $\quad 607-255-3990$

FAX: 607-255-4137

e-mail: aca7@cornell.edu

Paul M. Amirtharaj

Semiconductor Electronics Division

NIST

Gaithersburg, MD 20899

Tel: $\quad 301-975-5974$

FAX:

e-mail: paul.amirtharaj@nist.gov

Steven Arrivo

Optical Technology Division

NIST

Gaithersburg, MD 20899

Tel: $\quad 301-975-4713$

FAX:

e-mail: steveb.arrivo@nist.gov

Naira Maria Balzaretti

Ceramics Division

NIST

Gaithersburg, MD 20899

Tel: $\quad 301-975-5601$

FAX:

e-mail: naira.balzaretti@nist.gov

Michael I. Bell

Code 6680

Naval Research Laboratory

Washington, DC 20375

Tel: $\quad 202-404-7543$

FAX: 202-767-4868

e-mail: michael.bell@nrl.navy.mil

Girsh Blumberg

Department of Physics at Urbana-Champaign

University of Illinois

1110 W. Green Street

Urbana, IL 61801

Tel: $\quad 217-244-8038$

FAX: 217-244-8544

e-mail: blumberg@uiuc.edu
Linda M. Braun

Ceramics Division

NIST

Gaithersburg, MD 20899

Tel: $\quad 301-975-5777$

FAX:

e-mail: linda.braun@nist.gov

Richard R. Cavanagh

Surface and Microanalysis Science Division

NIST

Gaithersburg, MD 20899

Tel: $\quad 301-975-2368$

FAX:

e-mail: richard.cavanagh@nist.gov

Deane Chandler-Horowitz

Semiconductor Electronics Division

NIST

Gaithersburg, MD 20899

Tel: $\quad 301-975-2084$

FAX: 301-948-4081

e-mail: Deane.ChandlerHorowitz@nist.gov

Haridas Chandran

Department of Physics and Astronomy

Howard University

$22166^{\text {th }}$ Stret, N.W.

Washington, DC 20059

Tel: $\quad 202-806-4913$

FAX: $\quad 202-806-4429$

e-mail:

Steven J. Choquette

Analytical Chemistry Division

NIST

Gaithersburg, MD 20899

Tel: $\quad$ 301-975-3096

FAX:

e-mail: steven.choquette@nist.gov

Raju Datla

Optical Technology Division

NIST

Gaithersburg, MD 20899

Tel: $\quad 301-975-2131$

FAX:

email: raju.datla@nist.gov 
Krishna K. Deb

US Army Research Laboratory

Infrared Sensor Directorate

Fort Belvoir, VA 22060

Tel: $\quad$ 703-704-2024

FAX: 703-704-1345

e-mail: kdeb@nvl.army.mil

Sam Ditman

2800 Sykesville Road

Westminster, MD 21157-7626

Tel: $\quad 410-876-7835$

FAX:

e-mail:

Joy P. Dunkers

Polymers Division

NIST

Gaithersburg, MD 20899

Tel: $\quad 301-975-6841$

FAX: 301-947-0180

e-mail: joy.dunkers@nist.gov

Franklin J. Dunmore

Optical Technology Division

NIST

Gaithersburg, MD 20899

Tel: $\quad 975-2328$

FAX: 301-840-8551

e-mail: dunmore@garnet.nist.gov

Bruno M. Fanconi

Polymers Division

NIST

Gaithersburg, MD 20899

Tel: $\quad 301-975-6769$

FAX:

e-mail: bruno.fanconi@nist.gov

Bruce O. Fowler

Polymers Division

NIST

Gaithersburg, MD 20899

Tel: $\quad 301-975-6834$

FAX: 301-963-9143

e-mail: bruce.fowler@nist.gov
Adolfas Gaigalas

Biotechnology Division

NIST

Gaithersburg, MD 20899

Tel: $\quad 301-975-2873$

FAX:

e-mail: adolfas.gaigals@nist.gov

Daniel G. Gammon

Code 6876

Naval Research Laboratory

Washington, DC 20375

Tel: $\quad 202-404-4533$

FAX: 202-767-1165

e-mail: gammon@bloch.nrl.navy.mil

Richard S. Gates

Ceramics Division

NIST

Gaithersburg, MD 20899

Tel: $\quad 301-975-3677$

FAX: $\quad 301-990-8729$

e-mail: richard.gates@nist.gov

Lori S. Goldner

Optical Technology Division

NIST

Gaithersburg, MD 20899

Tel: $\quad 301-975-3792$

FAX: 301-670-6249

e-mail: lori@bruce.nist.gov

Tonya M. Herne

Process Measurements Division

NIST

Gaithersburg, MD 20899

Tel: $\quad 301-975-5153$

FAX: 301-869-5924

e-mail: therne@nist.gov

Wilbur S. Hurst

Process Measurements Division

NIST

Gaithersburg, MD 20899

Tel: $\quad 301-975-4814$

FAX: 301-869-5924

e-mail:whurst@nist.gov 
Christopher A. Kendziora

Code 6653

Naval Research Laboratory

Washington, DC 20375

Tel: 202-767-2414

FAX: 202-767-6980

e-mail: Kendzior@ccfsun.nrl.navy.mil

M. Anne Leugers

Spectroscopy Laboratory

1897 Building

Dow Chemical Co.

Midland, MI 48667

Tel: $\quad$ 517-686-9865

FAX:

e-mail:

James E. Maslar

Process Measurements Division

NIST

Gaithersburg, MD 20899

Tel: $\quad 301-975-4182$

FAX: 301-869-5924

e-mail: jmaslar@nist.gov

Norman G. Moll

667 Building

Dow Chemical Co.

Midland, MI 48667

Tel: $\quad$ 517-636-3748

FAX: $517-638-7347$

e-mail: normmoll@dow.com

Vyacheslav B. Podobedov

Optical Technology Division

NIST

Gaithersburg, MD 20899

Tel:

FAX: $\quad 301-975-3038$

e-mail:

John F. Rabolt

Materials Science Department

University of Delaware

Newark, DE 19716

Tel: $\quad 302-831-4476$

FAX: 302-831-4545

e-mail: rabolt@udel.edu
Joseph P. Rice

Optical Technology Division

NIST

Gaithersburg, MD 20899

Tel: $\quad 301-975-2133$

FAX:

e-mail: joe.rice@nist.gov

Lawrence D. Rotter

Ceramics Division

NIST

Gaithersburg, MD 20899

Tel: $\quad 301-975-6603$

FAX: 301-990-8729

e-mail:1rotter@nist.gov

John R. Rumble, Jr.

Standard Reference Data Program

NIST

Gaithersburg, MD 20899

Tel: $\quad 301-975-2200$

FAX:

e-mail: john.rumble@nist.gov

Eric L. Shirley

Optical Technology Division

NIST

Gaithersburg, MD 20899

Tel: $\quad 301-975-2349$

FAX:

e-mail: eric.shirley@nist.gov

Alfons Weber

Optical Technology Division

NIST

Gaithersburg, MD 20899

Tel: $\quad 301-975-2377$

FAX: 301-975-3038

e-mail: aweber@tiber.nist.gov 


